University of Louisville

ThinkIR: The University of Louisville's Institutional Repository

$8-2007$

\title{
A study of relationships of school climate, school culture, teacher efficacy, collective efficacy, teacher job satisfaction and intent to turnover in the context of year-round education calendars.
}

Joseph W. Mattingly 1953-

University of Louisville

Follow this and additional works at: https://ir.library.louisville.edu/etd

\section{Recommended Citation}

Mattingly, Joseph W. 1953-, "A study of relationships of school climate, school culture, teacher efficacy, collective efficacy, teacher job satisfaction and intent to turnover in the context of year-round education calendars." (2007). Electronic Theses and Dissertations. Paper 923.

https://doi.org/10.18297/etd/923

This Doctoral Dissertation is brought to you for free and open access by ThinkIR: The University of Louisville's Institutional Repository. It has been accepted for inclusion in Electronic Theses and Dissertations by an authorized administrator of ThinkIR: The University of Louisville's Institutional Repository. This title appears here courtesy of the author, who has retained all other copyrights. For more information, please contact thinkir@louisville.edu. 
A STUDY OF RELATIONSHIPS OF SCHOOL CLIMATE, SCHOOL CULTURE, TEACHER EFFICACY, COLLECTIVE EFFICACY, TEACHER JOB SATISFACTION AND INTENT TO TURNOVER IN THE CONTEXT OF YEAR-ROUND EDUCATION CALENDARS

By

Joseph W. Mattingly

M. Ed., University of Louisville, 1979

\author{
A Dissertation \\ Submitted to the Faculty of the \\ Graduate School of the University of Louisville \\ in Partial Fulfillment of the Requirements \\ for the Degree of
}

DOCTOR OF PHILOSOPHY

Educational Leadership, Foundations and

Human Resource Development

University of Louisville

Louisville, Kentucky

August 2007 
Copyright 2007 by Joseph W. Mattingly

All rights reserved 
A STUDY OF RELATIONSHIPS OF SCHOOL CLIMATE, SCHOOL CULTURE, TEACHER EFFICACY, COLLECTIVE EFFICACY, TEACHER JOB SATISFACTION AND INTENT TO TURNOVER IN THE CONTEXT OF YEAR ROUND EDUCATION CALENDARS

\author{
By \\ Joseph W. Mattingly \\ M.Ed., University of Louisville, 1978
}

A Dissertation Approved on

July 27, 2007

By the following Dissertation Committee:

Dr. Thomas G. Reio, Jr.

Dissertation Director

Dr. Mark W. F. Condon

Dr. Ann E. Larson

Dr. Patricia K. Leitsch

Dr. Randall L. Wells 


\section{DEDICATION}

This dissertation is dedicated to my wife and children and many special people who have provided me with focus and support throughout my life. Most importantly, I would like to express my sincere gratitude, appreciation, and love to my wife and best friend, Barbara Mattingly, for her constant understanding, patience, unconditional love, and valuable support throughout this process when there did not seem to be a light at the end of the tunnel and the world would seem to close in. Her strong belief in me and her constant encouragement have been invaluable.

My second level of strength came from my children: Megan E. Mattingly, Barrett A. Mattingly, and Elliot R. Mattingly. A Dad could not ask for three better children. You have made many sacrifices, and always had your own words of encouragement and inspiration to offer. You gave me motivation and kept me going and never wavered in your support of my educational pursuits. Thank you for your love, encouragement and always believing in me. To all of you: Two small, yet heartfelt and very genuine words, "Thank you!" I feel truly blessed to have each of you in my life and I am pleased to share this accomplishment with you. 


\section{ACKNOWLEDGEMENTS}

My long journey to the completion of my Doctoral degree began with years of stimulating coursework. It will end after years of collaborative work on this dissertation. Along the way, I have learned valuable lessons from my classmates and professors alike. In particular, I would like to thank the members of my committee, Dr. Thomas G. Reio, Jr., Dr. Mark F. Condon, Dr. Anne E. Larson, Dr. Pat Leitsch, and Dr. Randy L. Wells for their encouragement and honest feedback. I am deeply appreciative of your taking ownership of me and my study although none of you ever had me in a class. You have combed through each chapter and offered invaluable input. You used a gentle, yet firm approach to make me see areas of my study that could be strengthened. I am grateful for the many hours you have spent helping me complete my work.

I would especially like to thank Dr. Reio for your undaunted patience over the past two years as you guided me through this project and, professionally but firmly, telling me that it might be time now to finish! Not only have you been an excellent guide as my committee chair, but you have kept me calm and focused on the goal. You had a knack for transforming my anxieties into the confidence that I could finish this goal.

I express additional thanks to one of my subject matter experts, Ms. Cinda Sterns, who gave me valuable feedback and played a key role in the initial phase of this study, to other researchers who permitted me to use their ideas and finally, to all the participating school districts and teachers, especially the principals and teachers in my school district, who voluntarily agreed to be part of this study and made this study come true. 


\begin{abstract}
A STUDY OF RELATIONSHIPS OF SCHOOL CLIMATE, SCHOOL CULTURE, TEACHER EFFICACY, COLLECTIVE EFFICACY, TEACHER JOB SATISFACTION AND INTENT TO TURNOVER IN THE CONTEXT OF YEAR-ROUND EDUCATION CALENDARS
\end{abstract}

\author{
Joseph W. Mattingly
}

Committee Chairperson: Dr. Thomas G. Reio, Jr.

Educational Leadership, Foundations and Human Resource Development

August 2007

The goal of this study was to investigate the relationships between school climate, school culture, teacher efficacy, collective efficacy, teacher job satisfaction and intent to turnover in the context of year-round education (YRE) calendars. The research design of this study utilized an e-mail invitation to participate with a uniform resource locator (URL) embedded in the message to link participants to an internet-based questionnaire. The questionnaire was delivered to 1,254 teachers employed in nine participating school districts in Kentucky that operated on YRE school calendars. The response rate was $60 \%$. Teachers $(N=748)$ responded to an internet-based questionnaire consisting of six scales on the variables of school climate, school culture, teacher efficacy, collective efficacy, teacher job satisfaction and intent to turnover. An open-ended question from the internetbased questionnaire was examined as qualitative support for the findings from the quantitative data. Quantitative analysis involved examining descriptive statistics and 
correlations among research variables at the teacher level. Data were analyzed using Pearson's $r$ correlations, ANOVA and hierarchical regression analysis.

The findings demonstrated overall statistically significant positive correlations with study variables. High to moderate statistically significant positive correlations were found between school climate, school culture; teacher efficacy, collective efficacy, teacher job satisfaction and teacher intent to turnover.

After statistically controlling for demographic variables (ethnicity, gender, age, and years of YRE teaching experience, the addition of school climate subscale scores (collegial leadership, professional teacher behavior, achievement press, institutional vulnerability), school culture subscale scores (inquiry practice, teaching learning community and collective problem solving) into the second block of the regression equation resulted in an additional $16 \%$ of the variance; After statistically controlling for school climate subscale scores (collegial leadership, professional teacher behavior, achievement press, institutional vulnerability), school culture subscale scores (inquiry practice, teaching learning community and collective problem solving), the addition of teacher efficacy subscale scores (student engagement, teacher instructional practices and classroom management) into the third block of the regression equation resulted in an additional $18 \%(p<.05)$ of the variance; After statistically controlling for teacher efficacy subscale scores (student engagement, instructional practices and classroom management, the addition of collective competency subscale scores (group competence and task analysis) into the fourth block of the regression equation resulted in an additional $19 \%(p<.05)$ of the variance; and finally, after statistically controlling for collective efficacy subscale scores (group competence and task analysis), the addition of 
teacher job satisfaction subscale score (teacher job satisfaction global) into the fifth block of the regression equation resulted in $38 \%$ variance being predicted in the regression equation, a large size effect. Overall, the Conceptual Framework Model for Teacher Intent to Turnover in a YRE Context explained 38\% of the variance in the dependent variable teacher intent to turnover. The potential implications for theory and practice for school calendar designers and instructors are provided. 


\section{TABLE OF CONTENTS}

PAGE

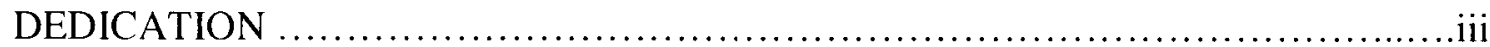

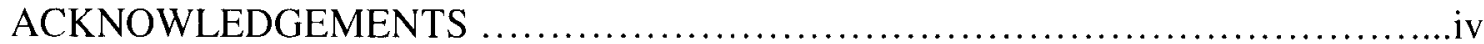

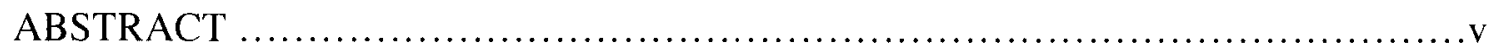

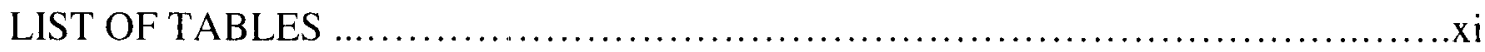

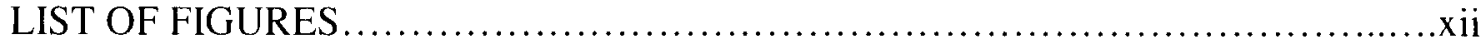

CHAPTERS $\quad$ PAGE

\section{INTRODUCTION}

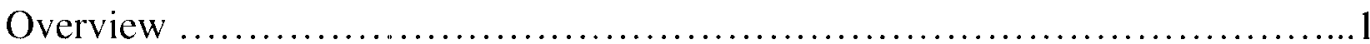

Background of the Problem ..............................................

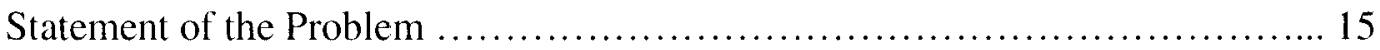

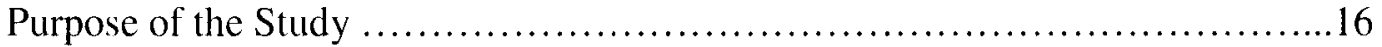

Research Questions . ............................................... 17

Assumptions ......................................................... 18

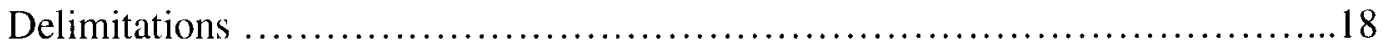

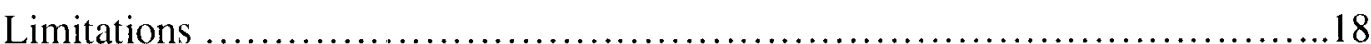

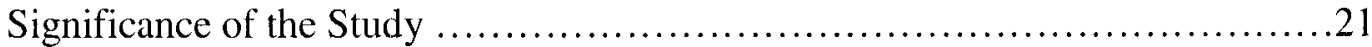

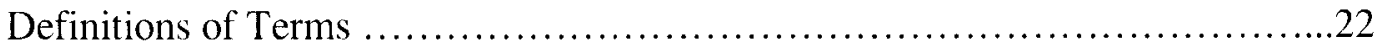

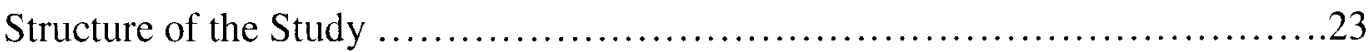




\section{LITERATURE REVIEW}

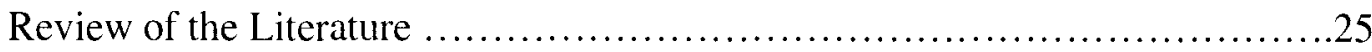

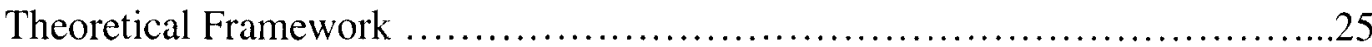

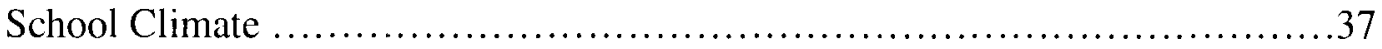

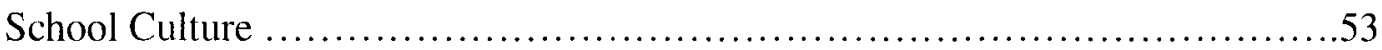

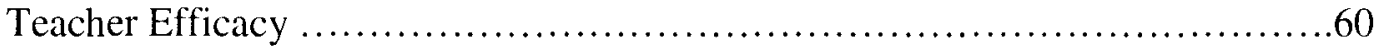

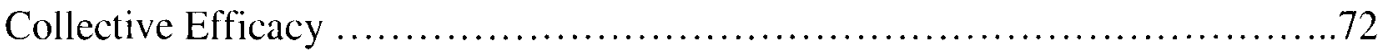

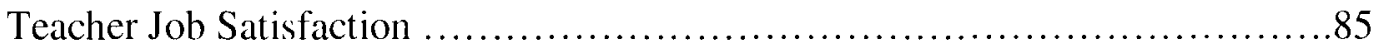

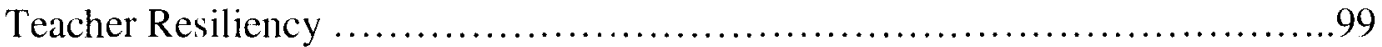

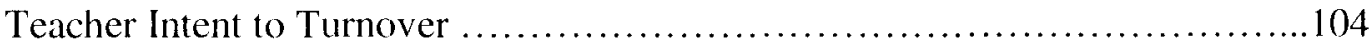

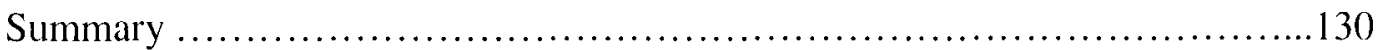

III. METHODS AND MATERIALS

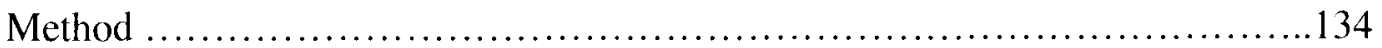

Participants ............................................................ 135

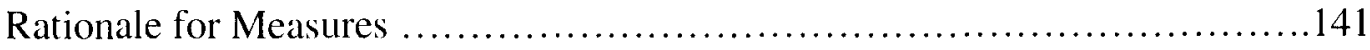

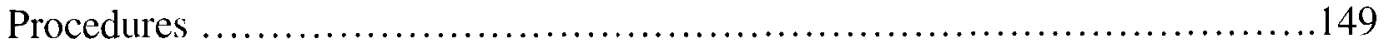

Data Analysis .................................................. 152

Answering the Research Questions .......................................153 


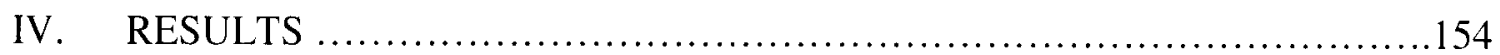

Descriptive Variables ............................................... 155

Questionnaire Distribution and Response Rates ..............................157

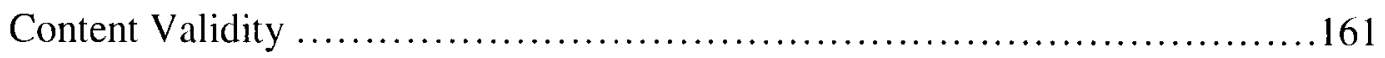

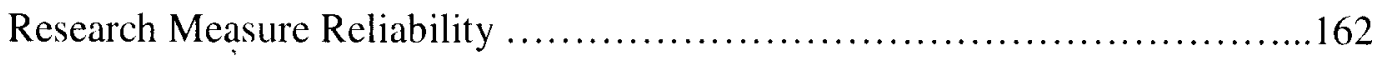

Inter-correlation among Demographic and Independent Variables ..............165

Answering Research Questions ...................................... 170

Answering Research Question \#1 ......................................173

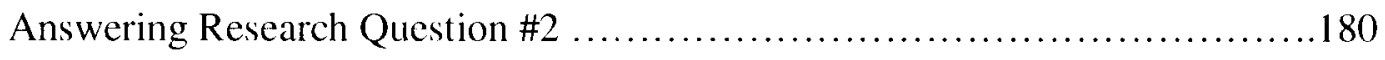

Answering Research Question \#3 ..................................... 182

Qualitative Data .................................................... 186

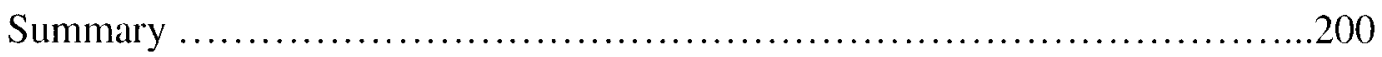

V. SUMMARY AND CONCLUSION

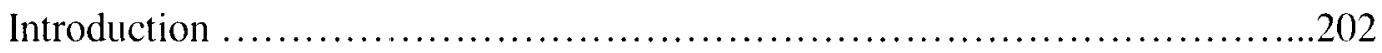

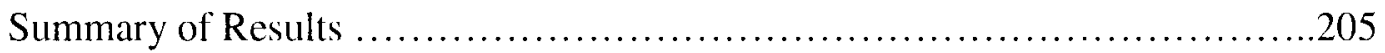

Discussion of Results According to the Three Research Questions .............207

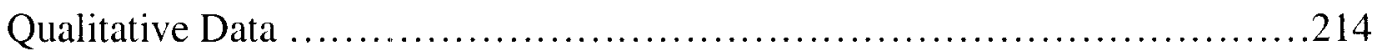

Limitations ......................................................... 216

Significance of the Study .............................................219

Suggestions for Additional Research ...................................220

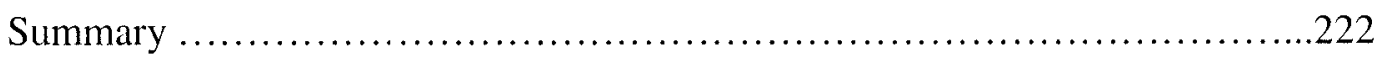

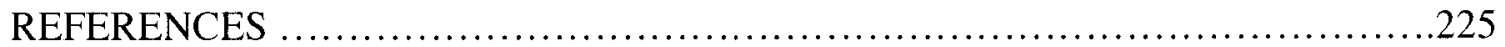

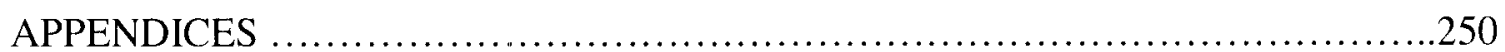

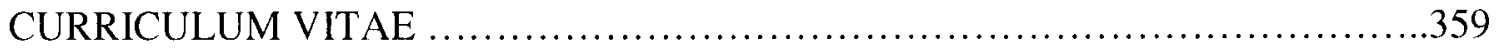




\section{LIST OF TABLES}

TABLE

PAGE

1. Study variables, measures, and subscales

2. Study Response Rate by Participating School Districts across Multiple

E-mail Invitations (Three) to Participate. 158

3. Two-Way ANOVA between E-Mail Wave Groups Analysis......................160

4. Means, Standard Deviations and Correlations of Demographic and

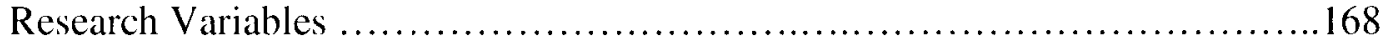

5. Research Questions and Statistical Measures .................................171

6. Varimax Rotation of Three Collective Efficacy Factors:

Collective Efficacy Subscales

7. Means, Standard Deviations, and Correlations for Demographic Items with Teachers' Years of Employment in a YRE calendar and Teacher Job Satisfaction with Teacher Intent to Turnover.

8. Summary of Hierarchical Regression Analysis for Variables Predicting Teacher Intent to Turnover (Intent to Turnover) Predicted by five Variables

9. Frequencies and Percentages of Participants' Responses Regarding Personal and Professional Impacts of the YRE Calendar

10. Frequencies and Percentages of Participants' Responses Regarding Personal Benefits of the YRE Calendar

11. Length of School Calendars around World 261

12. National Summary of Year-Round Education, 2005-2006 270 


\section{LIST OF FIGURES}

\section{FIGURE}

PAGE

1. Conceptual framework model for teacher intent to turnover in a YRE context 15

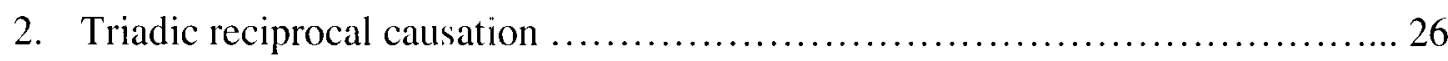

3. Sources of variations of self-efficacy ........................................ 29

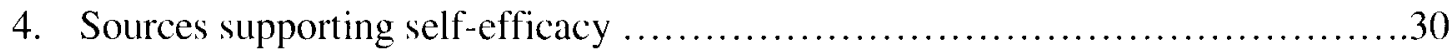

5. Conceptual framework model for teacher intent to turnover in a YRE context ............................................................ 184

6. Conceptual framework model for teacher intent to turnover in a

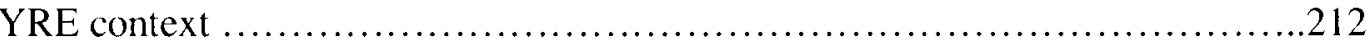

7. Traditional school calendar: Vacation and instructional days .................. 265

8. Year-round education school calendar: Vacation and instructional days ........ 266 


\section{CHAPTER I}

\section{INTRODUCTION}

\section{OVERVIEW}

America's public schools are facing a teacher turnover crisis due in part to the inability to recruit, retain, and fairly distribute the number of teachers that students and schools need (Colgan, 2004). Teachers are leaving for other professions or transferring to better schools and working conditions. School districts around the country are experimenting with different systems to develop better teacher working conditions as a measure to decrease teacher turnover. Individual schools and entire school districts are changing to a year-round education calendar (YRE; See Definitions, p. 23) as a productive approach to enhance student learning and teacher working conditions (McFadden, 2004). Teacher turnover has become a national emergency (Colgan, 2004; Loeb, Darling-Hammond \& Luczak, 2004; National Commission on Teaching and America's Future, 2005). As many as twenty-five percent (25\%) of teachers leave after one year and only fifty percent (50\%) remain after five years of service (Colgan, 2004; Darling-Hammond, 2000; White, 2005). When the nation's school year begins in 2008, more than 394,000 of the teachers will not return to the schools in which they taught last year. Replacing them constantly could cost almost $\$ 5$ billion (Alliance for Excellent Education, 2005; Cavanagh, 2005; Reese, 2004).

Teachers decide whether to remain at a school for a multitude of reasons. These reasons can be divided into the following four main categories: (1) characteristics of the 
job, including salary and working conditions; (2) alternative job opportunities; (3) teachers' own job and family preferences; and (4) school districts' personnel policies (Hanushek et al., 2003; Ingersoll, 2003; Loeb et al., 2004). Although some veteran teachers are changing jobs after many years in teaching, the majority of teachers leaving the classroom are new teachers (Ingersoll, 2003). Of the total number of teachers not returning to their schools this fall, more than 173,000 are leaving their profession altogether (Alliance for Excellent Education, 2005).

\section{Economic Cost of Teacher Turnover}

Breaking down the cost of teacher turnover further, the national cost estimate of replacing public school teachers who have dropped out of the profession is $\$ 2.2$ billion a year (Alliance for Excellent Education, 2005). The cost of replacing public school teachers who transfer schools for various reasons reaches $\$ 2.7$ billion annually (See APPENDIX A). For example, in Kentucky in 1999-2000, 1,650 of 42,842 public school teachers left the profession. At a cost estimate of $\$ 10,916$ per leaver, Kentucky expended $\$ 18,010,556$ to replace the teachers (Alliance for Excellent Education). In addition, Kentucky had 4,080 teachers transfer to other schools. Using the cost per transferring teacher of $\$ 10,913$, Kentucky expended $\$ 44,526,937$ to replace transfers and further expended a grand total of $\$ 62,537,493$ for replacing leavers and transfers (Alliance for Excellent Education). For other individual states, cost estimates of replacing teachers who leave the profession and teachers who transfer to other schools range from $\$ 62$ million in Kentucky to $\$ 504$ million dollars for a large state like Texas (Alliance for Excellent Education; Loeb et al., 2004; See APPENDIX A). 


\section{Loss of Instructional Expertise}

The price of losing teachers, particularly those who have just begun their teaching careers, is enormous in terms of economics; it is also costly in terms of the quality of education provided students who lose the value of being taught by experienced teachers (Cavanagh, 2005; Loeb et al., 2004; Public Policy Institute of California, 2006). High rates of teacher turnover greatly impact the total health (Hoy \& Woolfolk, 1993) of the school. Economic impacts include the following: recruiting, advertising, interviewing, hiring, training new teachers, and lost investment in professional development. Educational impacts include the loss of the following: improved teacher skills, curriculum knowledge, experienced teachers overburdened by needs of inexperienced colleagues, community within school and with parents, lost continuity and stability for students, and potentially reduced student achievement due to the loss in teacher experience and expertise (ASCD, 2004; Loeb et al., 2004; Public Policy Institute of California, 2006).

\section{Teacher Intent to Turnover in the Context of Year-Round Education Calendars}

The study of job satisfaction and its effects have been of concern and of interest to organizations and researchers since Hoppock (1935) first study of the topic. Studies have found that the lack of satisfaction can have serious consequences. One is the intent to turnover (Brayfield \& Crockett, 1955; Porter \& Steers, 1973; Vroom, 1964), which can lead to the second, the actual turnover event (Lambert et al., 2001). Implications of intent to turnover may domino into other human resource concerns including quality of service, productivity and ultimately overall organizational success (Test et al., 2003). These consequences may lead to costly training and recruitment issues and in addition may 
affect service delivery and productivity (Meyer \& Allen, 1997). Since job satisfaction and turnover are important factors in nonprofit organizational effectiveness (Sowa et al., 2004), understanding how to prevent these occurrences is why organizational leaders and researchers want to know more about the constructs of job satisfaction and intent to turnover and their potential relationship. There is ample research on employee intent to turnover in the business and industrial communities (Dore, 2005; Provasnik \& Dorfman, 2005; Watlington et al., 2004; Dee, 2002). There has been limited research of teacher intent to turnover in the traditional school setting (Guin, 2004, Quality Counts, 2003). However, teacher intent to turnover (intent to leave one's present position in an organization within a given time period; Dore, 2005) has not been examined in the YRE context. From the perspective of YRE calendar reform, teacher intent to turnover has not been a prevalent area of research. YRE calendars are known for breaking up the long summer break into more frequent shorter breaks throughout the school year. YRE advocates have touted the YRE modified calendar (See APPENDIX B) as providing improved working conditions and satisfaction for teachers because YRE allows greater calendar flexibility (Anderson \& Walberg, 1993), time for planning, reflection and increased teacher motivation (Haser \& Nasser, 2005). YRE has reduced teacher stress (Nasser \& Haser, 2002; McFadden, 2004; Worthen \& Zsiray, 1993), increased teacher attendance (Glines, 1994; Worthen \& Zsiray, 1993), decreased disciplinary referrals (Venable, 1997), provided preparation time (especially helpful for novice teachers), relieved both personal and inter-personal tensions, paved the way for more conversation and reflection about teaching and learning and provide additional part-time employment opportunities (Sheane \& Others, 1994) during intersessions (Ballinger, 1999, 2000; 
Donahue, 1997; Nicky, 1998; Shields \& Oberg, 1999). In addition, research suggests that YRE teachers miss fewer work days, request fewer transfers and remain longer in the profession than teachers working in the traditional calendar (Dejarnett, 1994; McFadden, 2004; See APPENDIX C). The YRE calendar is a school calendar strategy that provides a more balanced distribution of instruction and vacation days for teachers and students. The YRE calendar reduces the long summer break by re-apportioning those days throughout the school year; allows for more frequent vacation breaks; and limits the long periods of in-session instructional days.

Following more research into teacher intent to turnover and working environment satisfaction, educators find that research has linked the effect of school culture and school climate with high teacher retention (Fritts-Scott, 2005; Haser \& Nasser, 2005). YRE studies have reported preliminary findings that YRE calendar reform has positive effects on culture and climate (Fritts-Scott, 2005; Haser \& Nasser, 2005; Kennedy \& Wilcher, 1998; Kneese, 2000; Speck, 2002) in schools for both students and teachers (Boyles, 1993; Donahue, 1997; Worthen \& Zsiray, 1993). Teachers who have experienced the YRE calendar in their work environment reported positive impacts on both their teaching and personal life in general (Boyles, 1993; Donahue, 1997). Worthen \& Zsiray (1993) report that teachers on YRE calendars when compared to teachers working in a traditional calendar setting have somewhat better attitudes toward school, exhibit sixteen ( $16 \%$ ) percent less absenteeism, feel 'more professional', better rewarded financially, and report greater stress while in session, but less "burnout" across the year. If educators knew more about what climate and cultural strategies could be designed to alleviate the teacher intent to turnover, schools could experience greater stability in staffing and 
instruction. Because YRE calendar reform has been reported as having a positive impact on teacher working conditions (Fritts-Scott, 2005) and job satisfaction, further research on the relationship between YRE calendar reform and intent to turnover may provide useful new information to lessen teacher turnover.

\section{Background of the Problem}

\section{Employee Turnover in the Business/Industry Context}

High or low employee turnover can be detrimental to the life and productivity levels of any organization (Branham, 2006). The strain of employee turnover can be much greater on smaller businesses than larger corporations as the percentage of turnover is higher in smaller businesses due to the smaller number of personnel employed (Bliss \& Associates, 2001; Pinkovitz et al., 2006). Turnover is a major concern for organizations that experience labor shortages and competitive pressures which make retention of key employees a strategic issue. New employees do not become fully productive until they have been trained and gain experience, a process that takes time. Nationally, the average annual employee turnover rate for all companies is twelve percent (12\%). One study found that seventy-five percent ( $75 \%$ ) of the demand for new employees is simply to replace workers who leave the company or industry rather than new growth (Pinkovitz et al., 2006).

\section{Intent to Turnover and Employee Work Conditions}

For virtually any business or organization, the employees' work conditions impact satisfaction and productivity. Studies have indicated that intent to turnover is the workrelated outcome most highly associated with work-family conflict and stress (Carlson, 2003). Employees, in general, maintain behavioral intentions regarding their decisions on 
intent to leave (turnover) their jobs or intent to stay or continue working for the company or organization. Intent to turnover has been found to be related to the level of employee job satisfaction in all business areas (Hackes \& Hamouz, 1996; Vallen, 1993).

\section{Intent to Turnover in the Educational Context}

In an educational context, research indicates that many teachers express dissatisfaction with their jobs (Ingersoll, 2001; Maddox, 1998; White, 2005). Research on teacher turnover has attempted to ascertain if proper induction, a supportive environment, physical attributes, organizational climate, and empowerment can curb the flight of teachers from the profession and increase the retention rate of quality teachers (Cavanagh, 2005; Loeb et al., 2005). Business and industry try to cultivate employee satisfaction through bonuses, promotion and material rewards. Schools on the other hand, have fewer avenues for such inducements. Many schools struggle to address poor working conditions. For instance, teachers report being isolated in classrooms with closed doors, denied basic materials to do their jobs and inundated with nonessential duties (Cavanagh, 2005; Loeb et al., 2005; Vail, 2005; Williamson, 2006). Leadership provides them with little input into the design and organization of schools and offers little opportunity for career advancement and individually selected professional growth (Leung, 2006; Maddox, 1998; Southeast Center for Teaching Quality, 2004).

When exploring teacher attitudes in traditional and YRE workplaces, YRE teachers generally have a more positive outlook toward YRE than teachers on the traditional, agrarian school calendar (Costa, 1987; Elsberry, 1992; Fardig, 1992; Loyd, 1991; Merino, 1983; Nygaard, 1974; Pelavin, 1978; Prohm \& Baenen, 1996). Shields \& Oberg (2000) reported that after having some experience with a YRE calendar, 95\% of 
the teachers preferred it to the traditional agrarian calendar. In several instances, teachers in YRE experience less stress and burn-out (Campbell, 1994; Kneese, 2000; Mutchler, 1993). A few studies reported improved attendance among the teachers; however, not at statistically significant levels. The increased teacher attendance may be attributed to more frequent vacation breaks that allow teachers to rejuvenate themselves (Mutchler, 1993; Shields \& Oberg, 2000). Although there are a number of benefits in a YRE calendar (Fritts-Scott, 2005; Haser \& Nasser, 2005; Kennedy \& Wilcher, 1998; Kneese, 2000; Speck, 2002), much research is needed to explore intent to turnover in the YRE context. If researchers can understand better the possible antecedents of intent to turnover, educational research and practice could benefit significantly.

\section{Antecedents to Teacher Intent to Turnover}

\section{School Climate}

When exploring the concept of teacher turnover, a discussion of the antecedents of turnover is warranted (Ray, 2005). The contextual antecedents of school climate and school culture and the psychological antecedents of teacher efficacy, collective efficacy and teacher job satisfaction figure prominently in examining teacher turnover or intent to turnover. School climate reflects the physical and psychological aspects of the school that are more susceptible to change and that provide the preconditions necessary for teaching and learning to take place (Tableman \& Herron, 2004). School climate refers to the sum of the values, cultures, safety practices, working, and organizational structures within a school that cause it to function and react in particular ways. Although no consistent agreement in the literature on the components of school climate or their importance exists, most researchers emphasize caring as a core element (Ray, 2005). However, some 
place safety foremost, defining school climate as an orderly environment in which the school staff and students feel valued and able to pursue the school's mission free from concerns about disruptions and safety. Several aspects of a school's physical and social environment comprise its climate. Researchers identified the following eight areas: 1) Appearance and physical plant, 2) Faculty relations, 3) Student interactions, 4) Leadership/decision making, 5) Disciplined environment, 6) Learning environment, 7) Attitude and culture, and 8) School-community relations (Tableman \& Herron, 2004). School climate resulting from working conditions is closely related to teacher turnover and difficulties in recruiting and retaining teachers. While teachers expressed that time and empowerment were central in their abilities to help increase student achievement (Curtis, 2005), a collegial atmosphere led by a principal with a strong instructional emphasis mattered most in teachers' decisions about whether or not to stay in the school in which they work. Teachers are clear about the working conditions they need to be successful with students. Given sufficient time and control over curriculum and instruction, teachers believe they can help students learn (Southeast Center for Teaching Quality, 2004). In terms of helping students learn, teachers reported that working conditions most directly associated with their classrooms were more important than leadership and facilities (Vail, 2005; Weiss, 2005).

School climate is an important predictor of student achievement (Berry \& Hirsch, 2005; Loeb et al., 2005; Southeast Center for Teaching Quality, 2004). Experienced teachers are more effective at raising student achievement, on average, than are novice teachers. Some studies indicate that declines in student achievement are associated with an increase in the proportion of new, inexperienced teachers resulting from teacher 
transfers and turnover (Loeb et al.; Nye et al., 2004). Increasing teacher retention may help not only to raise student achievement and school quality in general, but also address the current shortfall of fully credentialed teachers in public schools (Berry \& Hirsch, 2005; Public Policy Institute of California, 2006; Ray, 2005).

\section{School Culture}

Some educators use the terms "climate" and "culture" interchangeably, but researchers in the field say there is an important difference between the two. How staff members feel about their school is climate. Why they feel the way they do is determined by culture by the values and behavior of those in the school. Culture reflects the shared ideas, assumptions, values and beliefs that give an organization its identity and standard for expected behaviors (Peterson \& Brietzke, 1994; Tableman \& Herron, 2004; Weiss, 2005). The culture of the school can be viewed as the existence of interplay between three factors: the attitudes and beliefs of persons both inside the school and in the external environment, the cultural norms of the school, and the relationships between persons in the school. Just as the attitudes and beliefs of persons both inside and outside the school building may facilitate or impede change, the norms, or informal rules that govern behavior exert influence on change efforts. These norms are developed over time and are influenced by the attitudes and beliefs of those inside and outside the school. In turn, the norms define expectations regarding how things are to be done. Based on past experience, the culture provides a template for future action - a guide to determine how things are done and to be done (Tableman \& Herron, 2004). When queried on fifteen of eighteen survey questions about school climate and effectiveness, YRE staff reported a culture represented by a more positive attitude than did those teachers on a traditional 
calendar; however no significance tests of statistical significance were conducted (Prohm \& Baenen, 1996).

Teachers' perceptions of school culture reflect actual school conditions (Berry \& Hirsch, 2005; Loeb et al., 2005; Southeast Center for Teaching Quality, 2004; Vail, 2005; Weiss, 2005). Studies have found that teachers are prone to leave schools serving high proportions of low-achieving, low-income, and minority students for more economically and educationally advantaged schools; this departure causes a drain of experienced and effective teachers in these high demographic schools (Berry \& Hirsch, 2005, 2005; Cookson, 2005; Falch \& Strom, 2005; Loeb et al.; Liu \& Meyer, 2005; Ray, 2005; Viadero, 2005). In schools with very high teacher turnover rates, this turnover can pose a number of challenges, including lack of continuity in instruction, lack of adequate teaching expertise for making curriculum decisions and providing support and mentoring, and lost time and resources for replacement and training (Loeb et al.).

\section{Teacher Efficacy}

Teachers' sense of efficacy is a judgment about capabilities to influence student engagement and learning, even among those students who may be difficult or unmotivated (Peterson \& Brietzke, 1994; Vail, 2005). Efficacy, in psychological terms, is a person's perceived expectation of succeeding at a task or obtaining a valued outcome through personal effort (Lee, Dedrick \& Smith, 1991). Teachers with a strong sense of efficacy tend to exhibit greater levels of planning, organization, and enthusiasm. They spend more time teaching in areas where their sense of efficacy is higher, whereas teachers with lower efficacy tend to avoid subjects and topics. Teachers with a high sense of efficacy tend to be more open to new ideas, more willing to experiment with new 
methods to better meet the needs of their students, and more committed to teaching. Teachers with a high sense of efficacy persist when things do not go smoothly and are more resilient in the face of setbacks. They tend to be less critical of students who make errors and work longer with a struggling student (Ashton \& Webb, 1986; Coladarchi, 1992; Gibson \& Dembo, 1984; Tschannen-Moran \& Woolfolk Hoy, 2001).

In the context of YRE, research indicates the majority of teachers in YRE believe the calendar reform substantially enhances their sense of teaching efficacy (Shields \& Oberg, 2000; White, 2005; Worthen \& Zsiray, 1983). The opportunity for extended contracts and higher pay can reduce the need for teachers to moonlight in other jobs to earn extra money. Due to the frequency of instructional breaks on the YRE calendar, teachers exhibit improved morale and motivation (North Carolina Insight, 1997; Minnesota, 1999; Quinlan, George \& Emmett, 1987; Reese; Worthen \& Zsiray, 1983). Other benefits mentioned were less classroom stress, more time for relaxation and renewal, chance to reflect, time to regroup and reorganize instruction (Gandara, 1994; Schuyler, 1997).

\section{Collective Efficacy}

Teachers have a sense of efficacy in their own capabilities and also have collective efficacy beliefs about the staff, school or district in which they work. Collective efficacy concerns judgments people make about the group's level of competency (Bandura, 1997; Parker, 1994). These beliefs demonstrate that teachers have not only self-referent efficacy perceptions but also beliefs about the collective capability of the school staff. Such group-shared perceptions reflect a developing organizational attribute known as perceived collective efficacy (Bandura, 1997; Goddard, Hoy \& 
Woolfolk Hoy, 2000; Hoy, Sweetland \& Smith, 2002). Within any organization, the perceived collective efficacy represents the shared beliefs of group members concerning "the performance capability of a social system as a whole" (Bandura, 1997, p. 469). For schools, perceived collective efficacy refers to the judgment of teachers in a school that the faculty as a whole can organize and execute the courses of action required to have a positive effect on students. In the collective efficacy context, teachers in YRE programs believe the quality of instruction is better than in traditional programs due to the continuity of instruction and intersessions (Quinlan \& Emmett, 1987)

\section{Teacher Job Satisfaction}

Teacher job satisfaction describes the contentment an individual feels about the job. Job satisfaction is one of the most significant predictors of new teacher retention (Curtis, 2005; Smith \& Ingersoll, 2004). Satisfaction can be defined as an overall feeling about one's job, career or in terms of specific facets of the job or career (e.g., compensation, autonomy, coworkers) or it can be related to specific outcomes, such as productivity (Rice, Gentile \& McFarlin, 1991). For teachers, satisfaction with their career may have strong implications for student learning. Specifically, a teacher's career satisfaction with his or her career may influence the quality and stability of instruction given to students. Fundamental to determining teacher efficacy and satisfaction is the expectation teachers hold for their students. If students are regarded as having low ability or as being unable to learn, teachers tend to lower expectations of their own ability to teach them (Lee et al., 1991). Some researchers argue that teachers who do not feel supported in their work or school climate may be less motivated to do best work in the classroom (Ashton \& Webb, 1986; Dramstad, 2004; Ostroff, 1992). In addition, highly 
satisfied teachers are less likely to change schools or to leave the teaching profession as compared to those who are dissatisfied with many areas of their work life.

\section{Teacher Background Variables}

Certain demographic (ethnicity, gender and age) and employment variables (highest education degree, school placement, content area, teaching experience, teacher experience in a YRE calendar, salary) might have a relationship with intent to turnover. Ingersoll (2001) reports that elementary school teachers tend to be more likely to be highly satisfied with their working conditions than secondary school teachers. The analysis indicates teacher background characteristics such as specialty field and age account for a significant amount of turnover. However, in the YRE context, little defining research exploring the significance of particular teacher demographics upon intent to turnover effects has occurred.

Teachers report that the longer they work in the YRE calendar, the more they prefer it (Fardig, 1992; Loyd, 1991; Nygaard, 1974; Shields, 1996). YRE teacher turnover rate is low and staff daily attendance is high (Worthen \& Zsiray, 1983) Indeed, it was reported that teachers were absent considerably less on YRE schedules (Brekke, 1984; Worthen \& Zsiray, 1993). Quinlan et al. (1987) found preliminary evidence that YRE decreased teacher absences, although not at statistically significant levels. In the YRE context, teachers claim they are refreshed and experience less burn out. The staff finds that they spend less time transitioning (reviewing information and procedures). The shorter breaks promote continued enthusiasm, yet provide the necessary stability to the educational process (Goodwin, 2005). 


\section{Statement of the Problem}

Although there is considerable empirical research on school climate, school culture, teacher efficacy, collective efficacy and teacher job satisfaction, little is known about teachers' intent to turnover in the context of YRE education. The literature on YRE reports positive effects of the calendar reform on teacher stress and burnout, common workplace descriptors. Although the implementation of YRE to improve teaching and learning in school districts continues, empirical research to guide and understand YRE is lagging. The following conceptual model was developed to examine the systematic impact of school climate, school culture, teacher efficacy, collective efficacy and teacher job satisfaction on teacher intent to turnover in YRE schools.

\section{Conceptual Model}

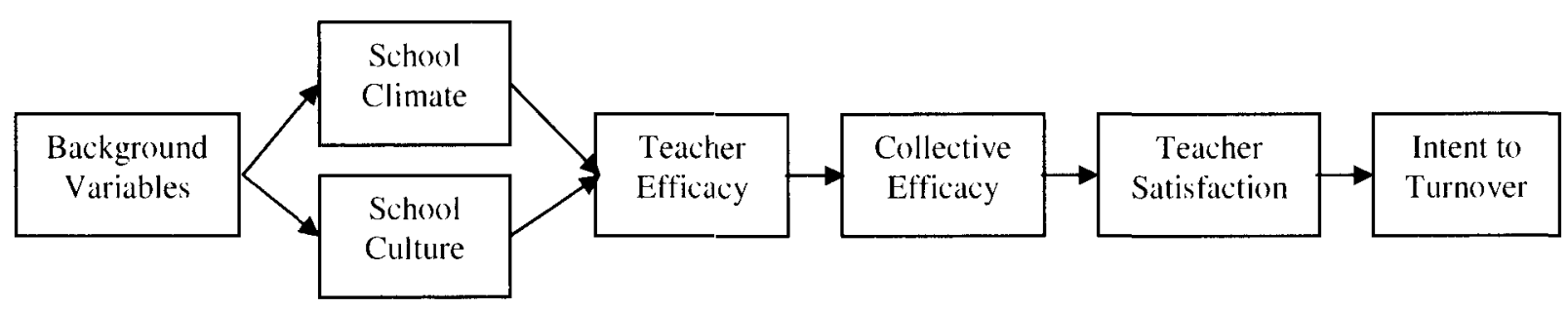

Figure 1. Conceptual Framework Model for Teacher Intent to Turnover in a YRE

Context.

The conceptual framework model in Figure 1 contains the graphical display of the independent variables of school climate, school culture, teacher efficacy, collective efficacy, teacher job satisfaction and the dependent variable of teacher intent to turnover. The framework is based on the expectation that the school climate and school culture have a predictive relationship with teacher efficacy, collective efficacy, teachers' job 
satisfaction and intent to turnover. This researcher accepts that school climate and school culture are to some degree affected by outside global, national, and state socio-cultural and socio-political influences. National educational initiatives like No Child Left Behind and state educational reforms like the Kentucky Education Reform Act with its high stakes assessment accountability exert considerable pressures on school culture and school climate within the state and local policy context. However, for the scope of this study, the framework of school culture and school climate will focus on the culture and climate resulting from interactions within the relationships of the local schools and districts. The study will analyze the culture and climate in the context of surveying teacher perceptions of their interactions with colleagues, students, parents and the school community at large. To analyze school culture and school climate through the perceptions of other school culture and school climate stakeholders like students, parents, and the community at large would be beyond the scope of this study and recommended as further study regarding teacher intent to turnover.

The conceptual figure depicts how school climate, school culture, teacher efficacy, collective efficacy, teacher job satisfaction predict intent to turnover after controlling theoretically relevant demographic and background variables (gender, age, ethnicity, years of teaching, years of teaching in a YRE calendar, content area and salary and how each interact with the other.

\section{Purpose of the Study}

The purpose of this study is to examine the relationship of school climate, school culture, teacher efficacy, collective efficacy, teacher job satisfaction and teacher intent to turnover in a YRE calendar school context. While an impressive amount of research has 
been conducted in general public schools on traditional school calendars, limited research has been conducted concerning the attrition and retention of teachers who work within a YRE school calendar. Such data should allow researchers, administrators and practitioners a better understanding of how teachers perceive their jobs in the context of the YRE calendar and the likelihood that they do or do not intend to remain in their particular school or profession. This study expands on previous studies (Deever \& Shockley, 1975; Haser \& Nasser, 2003, Jones, 1992; Kochek, 1996; Sanders, 2001) by examining the unexplored relationships of school climate, school culture, teacher efficacy, collective efficacy, teacher job satisfaction and intent to turnover in the context of YRE.

\section{Research Questions}

The proposed research questions are as follows:

1. To what extent do school climate, school culture, teacher efficacy, collective efficacy, teacher job satisfaction relate to teacher intent to turnover in the YRE context?

2. To what extent does teachers' length of employment in a YRE calendar relate to teacher intent to turnover?

3. After controlling for the background variables (i.e., gender, age, years of YRE teaching experience), what is the unique contribution of school climate, school culture, teacher efficacy, collective efficacy, teacher job satisfaction and intent to turnover? 


\section{Assumptions}

1. All of the measuring scales in this study have appropriate content and construct validity.

2. The participants fully understood the instructions on the internet-based questionnaire and answered all questions honestly and appropriately (Schwarz, 1999).

\section{Delimitations}

Because this research's main purpose is to investigate the strength and direction of relationships between the contextual (school climate and school culture) and psychological variables (teacher efficacy, collective efficacy and job satisfaction) and intent to turnover, teacher performance and student academic achievement were not explored within the scope of this study.

\section{Limitations}

This study has four major limitations, which are common among research studies (Couper, 2001; Dillman, 2000; Fowler, 1993; Podsakoff, MacKenzie, Lee \& Podsakoff, 2003; Schaefer \& Dillman, 1998). The limitations are the following: limited causality and generalizability, self-report instruments, web survey data collection and response rates.

\section{Limited Causality and Generalizability}

This study used a non-experimental correlational research design, which limits influence of causality. The researcher used convenience sampling to obtain a sample of teachers in select Kentucky school districts who work in YRE school calendars. Thus, the lack of a true random sample limits the generalizability of the findings from this study. 


\section{Self-Report Instruments}

This study will rely on self-reported data; therefore, the data may possess elements of subjectivity. Although self-report instruments continue to be among the most widely used type of research measure, there is a tendency for people to report socially desirable responses (Couper, 2001; Podsakoff et al., 2003). Because data collection in this study relies on a self-report as well as a web-based questionnaire, common method variance may be a concern. Podsakoff et al. (2003) define common method variance as "variance that is attributable to the measurement method rather than to the constructs the measures represent" (p. 879). According to the authors, one recommendation for controlling common method variance is by using more than one method of data collection. The use of multiple collection methods was not feasible in the present study due to the limited access to survey participants. Thus, the possibility of inflated correlations between variables may not be eliminated, but other recommendations were utilized to control for common method variance. First, the anonymity strategy was used. Secondly, the researcher reduced evaluation apprehension by assuring respondents there was no right or wrong answer and encouraged them to answer each question as honestly as possible.

\section{Web Survey Data Collection}

Web-based questionnaires offer the possibility of very rapid surveying, an attribute well documented by past research (Dillman, 2000; Schaefer \& Dillman, 1998). Although web-based questionnaires are regarded as the new survey technology, there are many possible drawbacks that need to be explored (Couper, 2001). Computer literacy varies greatly among people, as does the processing power of their computers; thus web- 
based questionnaire data collection may limit the survey population to only those who have Internet access (Couper, 2000). However, this problem is often overcome by limiting the study to those with access to the Internet (Couper, 2001). It should be noted that the population in this study consists of Kentucky teachers who have universal access to e-mail and the Internet via the Kentucky Educational Technology System.

Dillman (2000) and Schaefer \& Dillman (1998) report that screen configurations and connection speeds also influence how people can access and respond to web surveys. In addition, the means of displaying questions on computer screens may have a profound effect on whether some people are able and/or willing to answer survey questions accurately. To effectively implement a web-based questionnaire, it is important to recognize the potential for technical problems and to carefully plan a means to overcome these problems if they are to arise (Shannon, Johnson, Searcy \& Lott, 2002). The development of the web-based questionnaire may impact the way it is received and viewed by the subjects. It is important to be aware that teachers will be operating on computers with (1) varying modem and internet connection speeds, (2) a host of hardware and software settings, and (3) browser types and versions; therefore, the webbased questionnaire should be designed and developed accordingly (Couper, 2001). Because of these differences, it is necessary to keep the file size of the web-based questionnaire small to decrease the time it takes for teachers to download web pages (Best \& Krueger, 2002; Dillman, 2000; Ilieva, Baron, \& Healey, 2002).

\section{Response Rates}

An additional concern with the web-based questionnaire is a lowered participant response rate than mail surveys. Some studies have reported response rates for Web 
surveys ranging from seven to forty percent (Cook, Heath, \& Thompson, 2000; Shannon \& Bradshaw, 2002; Simsek \& Viega, 2001). The researcher followed Dillman (2000) recommendations to optimize the response rate.

\section{Diversity of Participants}

A concern exists about the diversity of potential participants within the target school districts. The ratio of African-American teachers to Caucasian teachers in the target pool may cause difficulty in generalization of study results across more urban, diversified schools and school districts.

\section{Significance of the Study}

The results of this study may be important to a number of educational stakeholders such as school boards, administrators, teachers, parents and local policy makers. Policy implications abound with the issues of teacher working conditions (Schuyler, 1997). Acknowledging and addressing this intent to turnover issue is particularly important, given the disproportionate impact teacher turnover has on lowincome and minority students. Such knowledge has significant implications for both district and school-level policies. Increasing teachers' contentment and commitment to remain in the teaching profession is vital to local, state and national educational accountability. Research has shown that teacher intent to turnover is affected by the levels of job satisfaction and teacher efficacy (Kraut, 1975; Mobley, Horner, \& Hollingsworth, 1978; Prestholdt, Lane, \& Mathews, 1987; Steel \& Ovalle, 1984; Tett \& Meyer, 1993; Steel and Ovalle, 1984). The results of this investigation on the relationship of YRE calendar reform may reveal data suggesting school climate, school culture, teacher efficacy, collective efficacy, job satisfaction and intent to leave may be 
influenced by the context of the YRE workplace. Interest in YRE calendar reform as a possible strategy to counter the negative effects of intent to turnover of teachers exists among national educators, especially for single-track programs. The YRE calendar has gained recognition in California, Florida, Utah and Kentucky as a facet of calendar reform. Although implemented in many school districts in Kentucky and around the nation, limited research on the possible links of YRE calendar reform to intent to turnover exists (See Appendix C).

\section{Definitions of Terms}

1. Attrition: Teachers leaving the profession, not renewing professional contracts (August \& Waltman, 2004.

2. School Climate: The social atmosphere of a setting or "learning environment" in which students have different experiences, depending upon the protocols set by the teachers and administrators (Hoy, Smith \& Sweetland, 2002).

3. Collective Efficacy: Teachers' belief about the collective capability of a group of teachers to influence student achievement (Goddard, LoGerfo \& Hoy, 2004).

4. School Culture: Refers to the sum of the values, cultures, safety practices, and organizational structures within a school that cause it to function and react in particular ways (Tableman \& Herron, 2004).

5. Teacher Efficacy: the belief or judgment teachers have about their abilities to perform an instructional activity or those internal factors that affect teacher behaviors, on-going interaction between teachers and students that influence 
choice of actions and conduct of teachers in classrooms (Ashton \& Webb, 1986; Gibson \& Dembo, 1984).

6. Intent to Turnover: Intent to leave one's present position in an organization within a given time period (Dore, 2005).

7. Retention: Retaining employees/teachers within the organization (Smith \& Ingersoll, 2004).

8. Social cognitive theory: A framework for understanding, predicting, and changing human behavior. The theory identifies human behavior as an interaction of personal factors, behavior, and the environment (Bandura 1977; Bandura 1986).

9. Teacher satisfaction: Degree to which people like their jobs, a general attitude toward the job, the difference between the amount of rewards employees receive and the amount they believe they should receive (Brenner, 2004).

10. Year-Round Education (YRE): YRE is used to describe single-track, multitrack, and extended year approaches to school calendars where vacations are spaced at various intervals throughout the year instead of combined into one long summer vacation. This study will use the term (YRE) to mean singletrack YRE (Glines, 1988).

\section{Structure of the Study}

This study is organized in the standard thesis format. Chapter I provides an overview of the study, significance of the study, statement of the problem, sub problems, delimitations, limitations of the study, and summary. Chapter II presents a review of the literature relevant to this study. The review is designed to examine the following 
components: Introduction to the YRE concept, the history of year-round education, school climate, school culture, teacher and collective efficacy, teacher job satisfaction and teacher intent to turnover. Chapter III was a restatement of the problem and a described the research method used to conduct the study. Chapter III included an introduction of this study, research questions, measures, rationale for measures, procedures, data analyses, reliability, generalizability, validity, timeline and chapter summary. Chapter IV presented research results. Chapter V presented a discussion of the results of the relationships between the variables explored in this study. 


\section{CHAPTER II}

\section{REVIEW OF LITERATURE}

This review of school climate, school culture, teacher efficacy, collective efficacy, teacher job satisfaction and teacher intent to turnover in the context of year-round education (YRE) calendar, includes summaries of theory, research and program description of information in the following categories: Theoretical framework, social cognitive theory, school climate; school culture; teacher efficacy, collective efficacy, teacher job satisfaction, teacher resilience and teacher intent to turnover (dependent variable).

\section{Theoretical Framework}

The theoretical framework model is an organized and systematic articulation of a set of statements related to questions in a discipline that are communicated in a meaningful whole (Bandura, 1986). Its purpose is to describe, explain, and prescribe under what conditions an action should occur (Bandura, 1977a, 1978, 1986, 1989). The theoretical framework for this study is grounded in the social cognitive theory (SCT (changed from Social Learning Theory by Bandura in 1986; Bandura, 1977, 1986; Institute for Dynamic Educational Advancement, 2006; Pajares, 2002; Wade \& Schneberger, 2006). The SCT identifies human behavior as an interaction of personal factors, behavior, and the environment and highlights the importance of observing and the notion of modeling the behaviors, attitudes, vicarious learning as a form of social learning (Bandura, 1977, 1986). In addition, Bandura also introduced several other 
important concepts, including reciprocal determinism, self-efficacy, and the idea that there can be a significant temporal variation in time lapse between cause and effect. This name change was also likely the result of an effort to further distance himself and his theory from the behaviorist approach. Bandura's work has stimulated an enormous amount of research on learning and behavior, and has been extremely fruitful in developing techniques for promoting behavior change. Jones (1989) reported that behavior varies from situation to situation. This variation may not necessarily mean that behavior is controlled by situations but rather that situations are construed the differently by the different people and thus the same set of stimuli may provoke different responses from different people or from the same person at different times (Institute for Dynamic Educational Advancement, 2006; Pajares, 2002; Wade \& Schneberger, 2006).

Using the SCT model in the context of instruction, the interaction between the teacher and behavior involves the influences of the teacher's thoughts and actions (See Figure 2). Bandura (1998) explains human behavior in relation to continuous reciprocal interaction between cognitive, behavioral, and environmental influences - called a triadic reciprocal causation (Figure 2).

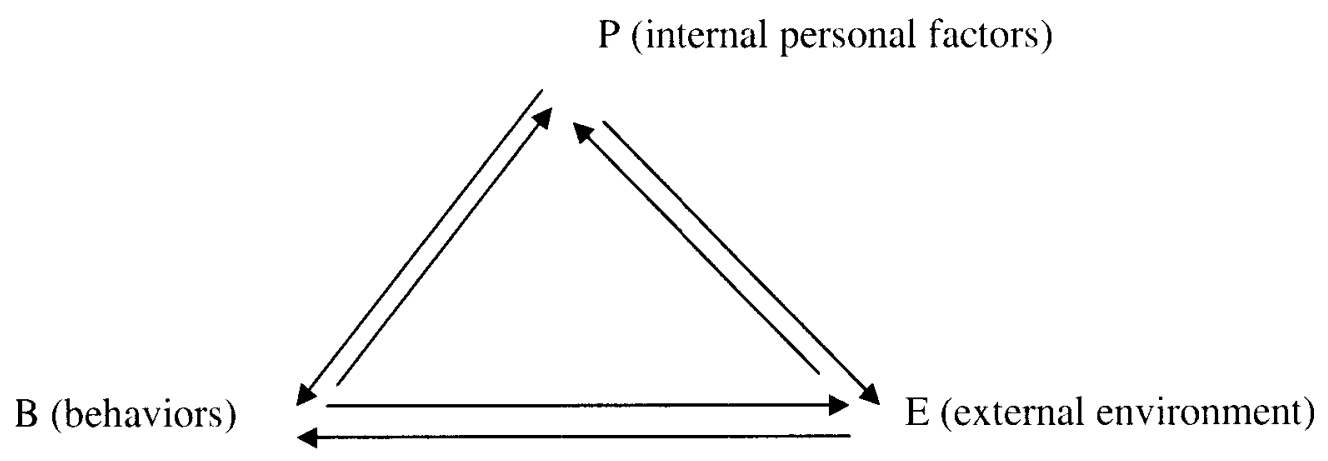


Figure 2. Triadic Reciprocal Causation: B represents behavior, P represents personal factors in the form of cognitive, affective, and biological events, and E represents the external environment (Bandura, 1986).

\section{Social Cognitive Theory: Self-Efficacy}

In the context of instruction, self-efficacy is the teacher's belief in capabilities to organize and execute actions needed to produce given goals within the classroom and school (Bandura, 1998). When referring to the relevance of self-efficacy, Bandura stated: By influencing the choice of activities and the motivational level, beliefs of personal efficacy made an important contribution to the acquisition of the knowledge on which skills are founded; it also supported efficient analytic thinking needed to search predictive knowledge from causally ambiguous environments. Beliefs of personal efficacy also regulated motivation by shaping aspirations and the outcomes expected for one's efforts (p. 35).

Bandura (1998), on the significance of self-efficacy, stated that motivational level and personal efficacy beliefs can make an important contribution to acquiring the knowledge needed for optimal skill development. Further, personal efficacy beliefs also regulate motivation by shaping aspirations and the outcomes expected from effort put forth. Not only did Bandura explore personal efficacy, but also the efficacy impact on groups. He defined the context of collective efficacy as "the group's shared belief in its conjoint capabilities to organize and execute the courses of action required to produce given levels of attainments" (p. 477). 
Wolters \& Daugherty $(2006 ; N=1,024)$ explored goal structures as a reflection of the teachers' motivational beliefs promoted by the prevailing instructional policies and procedures within an academic setting. Teachers' sense of efficacy refers to individuals' judgments or beliefs regarding their ability to accomplish critical instructional tasks. The relation between these constructs and differences on the basis of teaching experience and academic level were investigated. Teachers completed a self-report instrument via the Internet. Results indicated that teachers' sense of efficacy could be used to explain the classroom mastery goal structure they reported. Also, some aspects of teachers' sense of efficacy were greater for those with more teaching experience, whereas differences in goal structures were associated with academic level (Wolters \& Daugherty, 2006).

Efficacy beliefs vary in level, strength, and generality (Bandura, 1998) and each structure contains significant performance implications for instruction (See Figure 3). Level refers to the measure of task difficulty - simple demands, moderately difficult demands, or the most tough performance demands of teachers. The range of perceived efficacy is measured against levels of task demands made of teachers. What matters is not if the teacher believes she/he can perform the task, but the belief the task can be achieved on a regular basis (Bandura, 1998).

Caprara et al. $(2006 ; N=2,184)$ reported that strength refers to the continual teacher belief in capabilities to prevail over difficulties and obstacles that confront efficacy in instruction. Teachers' self-efficacy beliefs were examined as determinants of their job satisfaction and students' academic achievement. Teachers in 75 Italian junior high schools were administered self-report questionnaires to assess self-efficacy beliefs and their job satisfaction. Students' average final grades at the end of junior high school 
were collected in two subsequent scholastic years. The researchers employed a structural equation modeling analyses that corroborated a conceptual model in which teachers' personal efficacy beliefs affected their job satisfaction and students' academic achievement, controlling for previous levels of achievement (Caprara et al., 2006).

Bandura (1998) reported that fragile perceived self-efficacy is related to uncomfortable experiences, whereas strong perceived self-efficacy beliefs are related to stronger, successful efforts to prevail over challenging situations. The greater the selfefficacy belief, the more difficult tasks individuals will attempt, and with more probable success.

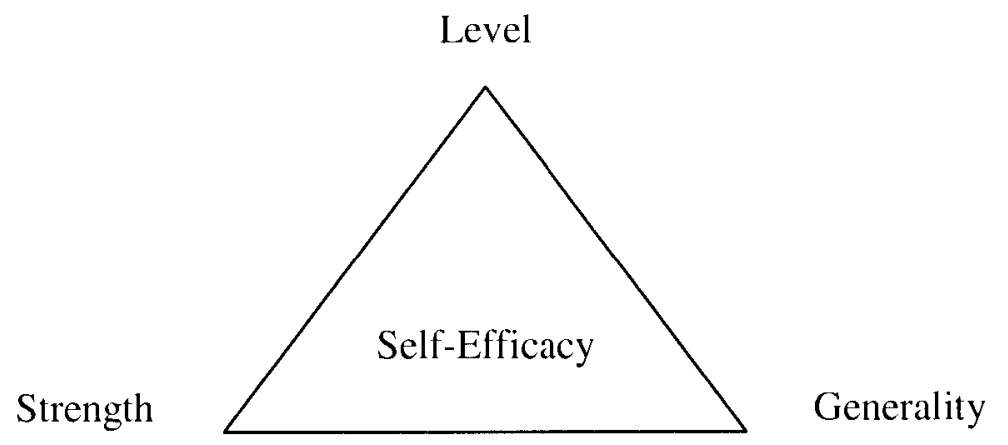

Figure 3. Sources of Variations of Self-Efficacy

Bandura (1998) defines generality as a teacher's own judgment across a wide range of activities or only in certain domains of functioning. Generality can vary in different dimensions, including the level of similarity of activities, the modalities in which capabilities are expressed (behavioral, cognitive, affective), qualitative characteristics of situations, and the characteristics of the individuals toward whom the behavior is directed (Bandura, 1998). Teachers' sense of efficacy is a judgment about capabilities to influence student engagement and learning, even among those students 
who may be difficult or unmotivated. Teachers with a high sense of efficacy tend to be more open to new ideas, more willing to experiment with new methods to better meet the needs of their students, and more committed to teaching. They persist when things do not go smoothly and are more resilient in the face of setbacks. And they tend to be less critical of students who make errors and to work longer with a student who is struggling (Ashton \& Webb, 1986; Coladarchi, 1992; Gibson \& Dembo, 1984; Tschannen-Moran \& Woolfolk Hoy, 2001). Teachers with a strong sense of efficacy tend to exhibit greater levels of planning, organization, and enthusiasm and spend more time teaching in areas where their sense of efficacy is higher, whereas teachers tend to avoid subjects and topics when efficacy is lower. For instance, teachers may consider themselves efficacious only in certain tasks (talking to people, writing papers, using a computer), but they might not feel as efficacious in other activities such as leading meetings or providing feedback.

\section{Sources of Self-Efficacy}

Self-efficacy is based on four sources of information: enactive mastery experience, vicarious experience, verbal persuasion, and physiological and affective states (Bandura, 1986; See Figure 4). These sources can change self-efficacy beliefs in teachers.

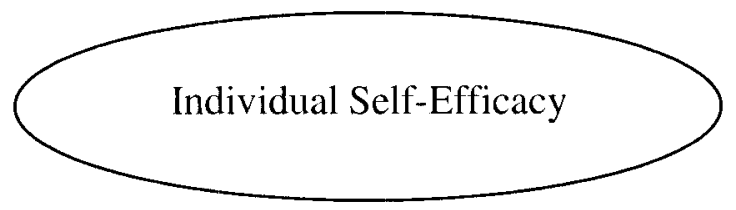

$\begin{array}{cccc}\text { Enactive Master } & \text { Vicarious } & \text { Verbal } & \text { Psychological } \\ \text { Experience } & \text { Learning } & \text { Persuasion } & \text { And Affective } \\ \text { (Number of } & \text { (Monitoring, } & \text { (Feedback) } & \text { States } \\ \text { Successes) } & \text { Observation, } & & \text { (Physical and } \\ & \text { Mentoring) } & & \text { Mood States) }\end{array}$

Figure 4. Sources Supporting Self-Efficacy (Bandura, 1986). 


\section{Enactive Mastery Experience}

In the context of instruction and teachers, enactive mastery experiences are the most influential source of information, because the source relies upon real-time classroom mastery experiences. Palmer (2006) reported that many preservice primary teachers initially have a low self-efficacy, or belief in their ability to teach science, but well-designed science education courses can produce significant positive changes in efficacy beliefs. However, the extent to which the belief changes are durable, or maintained over time, is yet to be established. The purpose of this study was to investigate the durability of changes in preservice primary teachers' self-efficacy beliefs. Their science teaching self-efficacy was measured at the beginning and end of a science methods course, and then after a delay period of nine months. Interviews were carried out 1 year after the end of the course. The results indicated that positive changes in selfefficacy occurred as a result of the course, and these high levels were still present after the delay period. Having an opportunity to teach science in primary school was a significant factor in consolidating efficacy levels after the methods course (Palmer, 2006).

Teachers' perceptions of successful teaching (mastery) raise expectations that their teaching will be proficient in the future (Bandura, 1997). On the other hand, teachers' perceptions that teaching has been a failure lowers teacher efficacy beliefs, contributing to the expectation that future performances will also be ineffectual. Interpretations of emotions and physiological arousal can add to the feeling of mastery or incompetence. For example, teachers' feelings of tension can be interpreted as anxiety and fear that failure is looming or as excitement (Bandura, 1977, 1997). The more 
successes teachers experience in teaching, the higher level of efficacy they exhibit; conversely, the more failures teachers experienced, the lower the level of perceived efficacy. When the teacher has had constant successes within the classroom or school, it is more likely that failures or mistakes will shape judgment of personal abilities. Thus, if a teacher has developed positive self-efficacy, it is more likely the teacher will be able to generalize this efficacy to other situations. Knowledge and strategies on certain matters will serve as tools to perform challenging instructional tasks, but the teacher has to also exercise control upon these knowledge and strategies consistently and persistently. For instance, if teacher tends to recall only the poorer performances, it is more likely that the teacher will underestimate personal efficacy belief (Bandura, 1977, 1997) and set expectations no higher than past poor performances.

\section{Vicarious Experience}

Vicarious experiences are those professional experiences in which someone (mentor, supervisor) else models a skill to help raise individual self-efficacy. Ross \& Gray $(2006 ; N=3,074)$ explored transformational leadership and teacher expectations that mediate between goals and actions. The most important of these expectations, teacher efficacy, refers to teacher beliefs that they will be able to bring about student learning. This study examined the mediating effects of teacher efficacy by comparing two models derived from Bandura's social-cognitive theory. Model A hypothesized that transformational leadership would contribute to teacher commitment to organizational values exclusively through collective teacher efficacy. Model B hypothesized that leadership would have direct effects on teacher commitment and indirect effects through teacher efficacy. Data from teachers in 218 elementary schools in a cross-validation 
sample design provided greater support for Model B than Model A. Transformational leadership had an impact on the collective teacher efficacy of the school; teacher efficacy alone predicted teacher commitment to community partnerships; and transformational leadership had direct and indirect effects on teacher commitment to school mission and commitment to professional learning community (Ross \& Gray, 2006). The more closely the observer identifies with the modeling of the skill, the stronger the impact on teacher's self-efficacy (Bandura, 1977, 1998). Self-efficacy appraisals are more sensitive to vicarious experiences when there is a lot of uncertainty in the teacher's capabilities. Perceived self-efficacy can be raised generally when the teacher has not had much prior experience (beginning teacher) or when the teacher observes other individuals performing well within the grade level and school and sense an expectation for group performance. Furthermore, the lack of direct knowledge capabilities (self-doubt) will make the teachers rely more on modeled indicators which allegedly have the desired competencies. For instance, a new teacher will benefit from shadowing an experienced teacher providing an effective instructional lesson. Modeling involving effective strategies will not only help a teacher's self-efficacy who has experienced a lack of efficacy in performance, but also, it may help self-assured teachers to increase self-efficacy through learning better and more effective models to do things (Bandura, 1998).

Vicarious experiences could also affect teachers' appraisals of self-efficacy through the affective states aroused by comparative self-evaluation. Self-depreciation and hopelessness may occur when new teachers compare themselves with superior teachers. However, a helpful comparison with equally talented teachers may provide positive selfregulation (Bandura, 1998) and a greater opportunity for individual success. The 
psychological modeling takes place in everyday environments such as schools or the workplace because these are the places where teachers professionally interact and observe each other (Bandura, 1997). This experimental study investigated whether selfefficacy perceptions pertaining to working with difficult-to-teach children could be increased for pre-service teachers using vicarious experience and verbal persuasion. 89 pre-service teachers were randomly assigned to groups. The experimental group was shown a 33-minute videotape describing and demonstrating effective behaviormanagement procedures, including supportive teacher testimonials and published research. A control group watched a placebo videotape of similar length detailing society's treatment of handicapped people. Data analyses indicated that the experimental group exceeded the control group on two of four self-efficacy measures. Results are discussed in terms of how enhancing teachers' self-efficacy perceptions might be used to "prime the pump" for consultation services (Bandura, 1997).

\section{Verbal Persuasion}

Verbal persuasion will help teachers affirm they possess abilities to perform successfully in the classroom. Social persuasion (by itself) is not strong enough to create enduring self-efficacy, but could contribute to successful performance if the appraisal is done realistically (Bandura, 1997). Persuasory efficacy information is frequently expressed in the evaluative feedback (formative and summative assessment) given to the teacher providing the instruction. When evaluative feedback emphasizes personal capabilities, efficacy beliefs elevate (Bandura, 1997). In addition, feedback referring to improved instruction because of diligent effort elevates perceived efficacy less than feedback implying that progress is due to natural ability. In other words, if teachers are 
told they have the ability because they gained it through diligent work, they will show a lower sense of efficacy as opposed to telling them that their progress shows they possess the ability without referring to their effort exerted (Bandura, 1997).

Dubay $(2001 ; N=108)$ explored teacher preparation programs responsible for preparing future teachers to use technology to support instruction in the P-12 classroom. Educational technologists agree that effective implementation of technology in the classroom by teachers requires that they have positive attitudes toward technology and confidence in their capabilities to use technology. The purpose of this study was to determine the effects of a verbal persuasion treatment, based upon Bandura's theory of self-efficacy, specifically designed to improve preservice teachers' attitudes and selfefficacy for educational technology. This study also examined the effect of computer ability on attitudes and self-efficacy for computer technology. Participants were preservice teachers enrolled in an elementary education technology course at a large midwestern university. The constructs of outcome expectancy, self-efficacy expectations, attitudes toward technology and computer ability were measured to address the hypotheses under investigation. A pretest/posttest generalized randomized block design was used to test the hypotheses. The blocking variable was level of computer ability. Participants were divided into two groups based upon their level of computer ability, then randomly assigned by ability level into either the treatment or control group. All participants in the study received modeling and hands-on technology activities. The treatment group received eight verbal persuasion messages via electronic mail. The messages consisted of statements encouraging students to believe that they were capable of implementing educational technology into their own teaching practices. Members of 
the treatment group were also persuaded to believe that they were capable of persevering when confronted with difficult technology situations. Participants in the control group received general course-related messages via electronic mail without any verbal persuasion statements. Results from the descriptive analysis procedures suggest that, in this sample, the treatment group had greater gain scores on the outcome expectancy, selfefficacy, and attitudes toward technology measures than did those students in the control group. The high and low-ability treatment groups had greater gain scores than the same ability control group levels. Findings from the inferential analysis procedures were inconclusive (Dubay, 2001).

Hagen et al. (1998; $N=89)$ investigated whether self-efficacy perceptions pertaining to working with difficult-to-teach children could be increased for pre-service teachers using vicarious experience and verbal persuasion. Pre-service teachers were randomly assigned to groups. The experimental group was shown a 33-minute videotape describing and demonstrating effective behavior-management procedures, including supportive teacher testimonials and published research. A control group watched a placebo videotape of similar length detailing society's treatment of handicapped people.

Data analyses indicated that the experimental group exceeded the control group on two of four self-efficacy measures Hagen et al., 1998).

\section{Physiological and Affective States}

Physiological state refers to teachers' relying "partly on information from their physiological state in judging their capabilities. Individuals read their somatic arousal in stressful situations as signs of being vulnerable to dysfunction" (Bandura, 1986, p. 401). Somatic indicators of personal efficacy usually involve physical accomplishments, health 
functioning, and coping with job stressors. For instance, an athlete performing physical activities requiring strength and stamina will probably pay attention to pains, fatigue and physical inefficacy. Furthermore, mood states can also affect judgment on efficacy. For instance, if a teacher has a negative mood, this mood will trigger thoughts of past failings, whereas a positive mood will bring about feelings of previous accomplishments in instruction (Bandura, 1997). Therefore, physiological and affective states are required to enhance physical status, reduce stress levels and negative emotional proclivities, and correct misinterpretations of bodily states (Bandura, 1997).

In sum, the four sources of self-efficacy can explain interpersonal self-efficacy in the context of instruction in the following way. First, enactive mastery experience will draw attention to the importance of previous positive teaching experiences of the teacher. Experienced teachers may possess more previous job experiences than a younger teacher. Second, vicarious experience will highlight the importance of observing, mentoring and supporting other teachers through modeling job performances. Third, verbal persuasion will provide the teacher with the support and evaluative feedback to believe in the ability to perform well at the classroom workplace. And fourth, physiological and affective states will stress the teacher's physiological and affective conditions such as health and stress. Therefore, interpersonal self-efficacy may be explained by feedback, support and interaction with others at the workplace (Bandura, 1997, 1986).

\section{School Climate}

Characteristics of schools, such as the physical structure of a school building and the interactions between students and teachers, are two diverse factors that both affect and help to define the broad concept of school climate. As stated in Chapter I, school 
climate refers to the sum of the values, cultures, safety practices, working, and organizational structures within a school that cause it to function and react in particular ways. It includes the explicit mission and policies expected to create positive relationships, attitudes or dispositions and perceptions (Talbert, 2002). Teaching practices, diversity, and the relationships among administrators, teachers, parents, and students contribute to school climate. The concept of school climate has become part of the standard rhetoric in contemporary discussions of school effectiveness and teacher retention (Hoy, Smith \& Sweetland, 2002; See APPENDIX D for school climate scale psychometrics). The study reported four critical aspects of school climate: 1) institutional vulnerability (the relationship between the school and community), 2) collegial leadership (the relationship between the principal and teachers), 3) professional teacher behavior (the relationship among teachers and staff), and 4) achievement press (teacher, parental, and principal pressure for achievement). A school's climate plays a direct and critical role in determining what the school is and what it might become (Hoy et al., 2002). The climate sets the tone for the school's approach to resolving problems, trust and mutual respect, attitudes, and generating new ideas. Good organizational climate involves establishing and maintaining satisfying relationships among coworkers and with families, providing opportunities through which individuals contribute to the program, incorporating appreciation and respect, and building feelings of accomplishment, such as making a difference in the lives of children and families (Klinker, Riley \& Roach, 2005). Poole and McPhee (1983) argued that the focus of school climate research must be on interaction processes, because climate is a function of the day-to-day practices in organizations and, simultaneously, a structure for interpreting or understanding specific 
events within the organization. Individuals within the organization come and go through transfer or leaving the profession, but the organizational climate, and the forces generating the collective feelings, remains. Researchers have used a variety of climate instruments to measure school climate.

In organizations, such as schools, the quality of the internal environment as experienced by members is generally referred to as climate (Hoy et al., 2002). Climate perceptions represent the apprehension of meaningful order in a perceiver's world and that perceived meaningfulness based on cues in that world is the basis for behavior. Each organizational climate is distinctive as having it own tempo, norms, traditions, styles and values (Hoy et al., 2002). These characteristics are determinants of member behavior. Others proposed that climate is dependent upon organizational structure attributes, such as locus of decision-making rules, hierarchical organization structure, size of organization, one's position in the hierarchy, leadership style (Litwin \& Stringer, 1968; Hall \& Lawler, 1969). Climate in this sense is generally assessed through organizational members' perceptions and descriptions of situational practices and procedures. Some more frequently used instruments are described in APPENDIX D.

As part of a larger study, Hoy et al. (2002) collected and analyzed data from a diverse and typical set of high schools in Ohio. Although procedures were not used to ensure a random sample from the population of high schools, care was taken to select urban, suburban, and rural schools from diverse geographic areas of the state. Only schools with fifteen or more faculty members were considered candidates for the study. One hundred forty-nine public high schools were contacted, but for a variety of reasons only 97 high schools agreed to participate (65\%). High schools were defined by grade 
span levels that included grades 9-12 and grades 10-12. Schools in the sample represented the entire range of socioeconomic status (SES); in fact, data from the Ohio Department of Education support the representativeness of the sample in terms of size, SES, and urban-rural balance. Participants complete the Organizational Climate Index. The analysis of survey results indicated the subscales of school climate to be only moderately related. Achievement press was positively related to both collegial leadership $(r=.32, p<.05)$ and professional teacher behavior $(r=.49, p<.05)$, but achievement press was unrelated to institutional vulnerability $(r=-.05$, n.s. $)$. Collegial leadership of the principal was positively associated with professional teacher behavior $(r=.27, p<$ .05). Moreover, collegial leadership was negatively related to institutional vulnerability ( $r$ $=-.45, p<.05)$

School climate is an important factor that affects student achievement and teacher retention (Bulach \& Berry, 2001; $N=1,163$; Instructional Improvement Survey). The Instructional Improvement Survey measures four culture and seven climate factors. The instrument consists of 96 items and has an overall reliability of .95 as measured by the Cronbach's alpha. Reliability on each of the subscales varies from .79 to .85 . Leadership behavior is measured in the following two culture and two climate factors: group atmosphere, group cooperation, instructional leadership, and discipline. Bulach and Berry's findings indicated that females and more experienced teachers were more positive about leadership behavior than males and teachers with less than ten years experience. In related research with the same instrument, Bulach and Peddle (2001) found a positive relationship .96 between leadership behavior associated with instruction and the overall culture and climate of the school. Their study involved twenty schools 
and $N=1,163$ teachers. Bulach \& Berry (2001) investigated the impact of teachers' gender, experience, number of years at a school, and degree status on the culture and climate of a school. Results indicated degree status did not make a difference. Female teachers, more experienced teachers, and teachers with more years at a school were more positive about the culture and climate of their school; however, teachers with 2-10 years' experience were the least positive about their schools' culture and climate. This study records that there is a need to improve levels of openness and trust in most schools, thereby improving their overall culture and climate. The data demonstrates that administrators need to spend more time with teachers who have 2-10 years of experience at a school. These teachers seem most at risk of leaving the profession, and with the teacher shortage, they cannot be ignored (Bulach \& Berry, 2001).

Moore \& Esselman (1994) found a positive school atmosphere (focused on instruction), the reduction of barriers to effective teaching, and classroom-based decisionmaking to be a positive influences on personal efficacy. Reading achievement performance was significantly related to teacher perception of personal efficacy $(r=.35$, $p=.03)$, influence in decision-making $(r=.54, p=.001)$, positive school climate $(r=.76$, $p=.03)$, staff collegiality $(r=.58, p=.03)$, and minimum barriers to effective teaching ( $r$ $=.71, p=.03$ ). Results suggested that schools with historically poor achievement tend to have teachers who, as a group, report a poorer image of school atmosphere which contributes to poorer perceptions of teaching effectiveness. Further, the weak sense of efficacy is in part a function of the poor performance of the school's children. Entering teachers have substantially stronger personal efficacy than do long-term teachers (Dembo \& Gibson, 1984) but as time passes, sometime after the tenth year of experience, teacher 
efficacy begins to decline. This decline in the sense of teacher efficacy may lead to engagement of instructional practices and attitudes detrimental to student performance (Moore \& Esselman, 1994).

Saphier \& King (1985) found significant differences between teachers, administrators, and specialists on professional treatment by administrators $(F=3.66, p<$ .03 ), with administrators scoring the highest, support staff the next highest, and teachers the lowest when exploring school socioeconomic status (SES). Significant differences were found for all three subscales among all schools with different SES (teacher professionalism, $F=9.52, p<.001$; treatment of teachers, $F=8.68, p<.001$; and teacher collaboration, $F=1.57, p<.02$ ). Teachers with the most positive attitudes were from the high or low SES schools, while teachers with the least positive attitudes were from middle SES schools (Saphier \& King, 1985). Significant correlations were found between subscales (teacher professionalism and administrator professional treatment of teachers $(r$ $=.71, p<.001)$, teacher collaboration and administrator professional treatment of teachers $(r=.51, p<.001)$, teacher professionalism and teacher collaboration $(r=.61, p$ $<.001)$ and teacher efficacy, empowerment, and conceptual level. All three subscales were significantly correlated with personal teaching efficacy (teacher professionalism, $r$ $=.19, p<.001)$; administrator professional treatment of teachers, $r=.13, p<.006$; teacher collaboration, $r=.17, p<.006$ ) as well as five or six empowerment subscales. Subscale 11, administrator professional treatment of others, was significantly correlated to teaching efficacy $(r=.15, p<.00 \|)$.

Significant positive relationship exists between overall organizational climate of the school and 900 secondary school teachers' organizational commitment (Turan, 1998; 
$N=900$; Organizational Climate Description Questionnaire-Rutgers Secondary (OCDQRS); Organizational Commitment Questionnaire, Mowday et al., 1979; See APPENDIX D for scale psychometrics). The specific objective of this study was to determine the strength of the relationship between each dimension of the organizational climate (supportive principal behavior, directive principal behavior, engaged teacher behavior, frustrated teacher behavior and intimate teacher behavior as measured by the OCDQ-RS for secondary schools, and organizational commitment of teachers as measured by the Organizational Commitment Questionnaire. The existence of a statistically significant relationship was found in the following: (1) between overall organizational climate of schools and teacher's organizational commitment $(r=.78, p<.01)$; (2) between supportive leader behavior and teachers' organizational commitment $(r=.52, p<.01)$; (3) between engaged teacher behavior and teachers' organizational commitment $(r=.73$, $p<0.01)$; between direct leader behavior and teachers' organizational commitment $(r=-$ $.27, p<.05$ ); and between frustrated leader behavior and teachers' organizational commitment $(r=-.36, p<.05)$. The study confirms that the organizational climate of the school provides overall information about the nature of leadership behavior (supportive and directive) and teacher behavior (engaged, frustrated, and intimate) that can be use to describe the quality of life and teachers' identification with their schools (Turan, 1998).

Climate is an important influence on teaching efficacy; that a positive school atmosphere (focused on instruction), the reduction of barriers to effective teaching, and classroom-based decision-making each contributed to teachers' sense of efficacy (Moore \& Esselman, 1994; Teacher Efficacy Scale, Ruscoe et al., 1989. Tucker $(2003 ; N=260)$ investigated relationships between teacher job satisfaction and morale, work 
environment, and sense of teacher efficacy of teachers involved in school reform in an urban school district. The results indicated the relationships between teacher morale and job satisfaction $(r=.16, p=.0001)$; morale and robustness $(r=.44, p=.0001)$; morale and general teaching $(r=.19, p=.0001)$; morale and personal efficacy $(r=.17, p=$ $.0001)$; satisfaction and both teaching $(r=.14, p=.0001)$; satisfaction and personal $(r=$ $.17, p=.0001)$; total efficacy and morale $(r=.23, p=.0001)$ and total efficacy and satisfaction $(r=.22, p=.0001)$. Secondly, the study examined the status of teacher morale, job satisfaction, overall range of work environment perception and level of personal and teaching efficacy of teachers who work in urban classrooms. Participants were administered three surveys, responded to a single question indicating their level of morale and completed a demographic data form. The researcher chose three surveys: Purdue Teacher Opinionnaire (Bentley \& Rempel, 1980); Environmental Robustness Semantic Differential Scale (RSD; Licata \& Willower, 1978); and Teacher Efficacy Scale (Gibson \& Dembo, 1984). Respectively, the scales measured morale and satisfaction, work environment perceptions, and sense of personal and teaching efficacy. The demographic information reported school and teaching level, gender, years of teaching experience and age. Findings indicated that teachers had above average morale, were partially satisfied with working conditions, described their workplace environment as being moderately high in robustness, and had a higher degree of personal efficacy than general teaching efficacy. Environmental robustness had the strongest relationship with morale, satisfaction, and teachers' sense of efficacy. The researcher noted, as teachers increased their perception of robustness in the workplace environment, morale and efficacy increased as well. Of the three levels of teachers, high school teachers had higher 
levels of morale, perceived environment robustness and sense of efficacy than did middle school or elementary teachers (Tucker, 2004).

The Southeast Center of Teaching Quality (2004; $N=34,000$; Working Conditions for Teachers, North Carolina Professional Teaching Standards Commission) found results indicating the following findings: 1) Teachers' working conditions are significant and strong predictors of student achievement; 2) Leadership is critical to improving working conditions, but principals and teachers perceive these conditions very differently; 3) Teachers are generally satisfied with most aspects of their working conditions, and since 2002, their views about them are improving across North Carolina; and 4) Teachers, regardless of their background and experience, view working conditions similarly (The Southeast Center of Teaching Quality, 2004). They felt "trapped" in jobs that were no longer relevant to their future plans, which further increased their feelings of role alienation. Teachers could not live up to their internal sense of mission or do what they deeply believed that they had to impersonate individuals who were alien to them. This felt obligation to playact someone they were not and its subsequent feelings of selfbetrayal, anger, and guilt often induced a deep sense of self-estrangement in the respondents, particularly when informants did not feel any support in the work communities (Joffres \& Haughney, 2001).

Barth (2006) reported on the various forms of relationships among adults within the school. The forms of relationships might be categorized in four ways: parallel play, adversarial relationships, congenial relationships, and collegial relationships. Leadership can promote a culture of collegial relationships in the school. Barth reports that school leaders foster collegiality when they do the following: 
- State expectations explicitly. For instance, "I expect all of us to work together this year, share our craft knowledge, and help one another in whatever ways we can."

- Model collegiality. For instance, visibly join in cheering on others or have another principal observe a faculty meeting.

- Reward those who behave as colleagues. For instance, grant release time, recognition, space, materials, and funds to those who collaborate.

- Protect those who engage in these collegial behaviors (Barth, 2006, p. 32).

A precondition for doing anything to strengthen practice and improve a school is the existence of a collegial culture in which professionals talk about practice, share their craft knowledge, and observe and root for the success of one another. Without these attributes in place, no meaningful school or staff improvement-no staff or curriculum development, no teacher leadership, no student appraisal, no team teaching, no parent involvement, and no sustained change-is possible (Barth, 2006). Empowerment, recognition, satisfaction, and success in our work will never stem from going it alone as a masterful teacher, principal, or student, no matter how accomplished the teacher is. Empowerment, recognition, satisfaction, and success come only from being an active participant within a masterful group--a group of colleagues in a collaborative culture.

Daugherty et al. (2005) reported that principals have the power, authority, and position to impact the climate of the school, but many lack the feedback to improve. Because schools have become very complex organizations, principals must move beyond occasional brilliant flashes to methods of continuous improvement. The variables associated with improved student achievement have been a focus of researchers for many years. The researchers investigated the relationships between selected dimensions of 
leadership and measures of school climate. In addition, principals' perceptions of their own leadership styles were compared with teachers' perceptions of their principals' leadership styles. The study included 31 elementary schools; therefore, $N=31$ principals and $N=155$ teachers (rive teachers per school) were involved. Teachers completed three instruments: Leader Behavior Analysis II (LBAII), Leader Effectiveness Scale (EFF), and Staff Development and School Climate Assessment Questionnaire (SDSCAQ). Pearson's $r$ correlations were calculated to determine the relationships between variables for the study. The unit of analysis is the school; therefore, the number of observation is 31- the number of schools in the study. Statistically significant positive relationships were established between teachers' perceptions of their principals' Effectiveness Scores and all six climate scores: Communication, Decision-Making, Innovation, Advocacy, Evaluation, and Staff Development. These obtained correlations suggest that school climate is directly linked to teachers' perceptions of a principal's effectiveness. For example, if teachers perceived that principals "used the most appropriate response for each situation" (high EFF scores) then they characterized the school to have good communications (.37), participatory decision-making (.37) and high levels of advocacy for teachers (.41). Indeed, each measure of school climate tended to be high if the principals were perceived to have high EFF and low if the principals were perceived to have low EFF. However, the corresponding correlations between the teachers' perceptions of principals' flexibility scores (FLX) and measures of school climate are all negative. The correlations with Communication Scale (.36) and Advocacy Scale (.40) are statistically significant. Thus, if principals are perceived to "select varying styles over a range of situations", then the teachers feel that the school has poor communication and 
weak teacher advocacy. The results indicate that the higher the teachers' perception of their principals' FLX score (flexibility), the lower their perception of teacher advocacy and the less effectiveness of communication within the building. Conversely, teachers perceive that less flexible principals lead buildings that share information, listen to concerns, and support teachers. The correlations between LBAII Flexibility and Effectiveness scores for principals' self-ratings and teachers' ratings of their principals on the same variables were not only insignificant; but also they approximate zero. For the various scenarios on the LBAII, principals' choices could not be predicted by teachers' perceptions of their principals' choices. Thus, the data indicates that the principals' selfratings of Effectiveness and Flexibility and the corresponding teachers' ratings are not related (Daugherty et al., 2005). If principals are highly skilled, they can develop feelings of trust, open communications, collegiality, and promote effective feedback. If principals are blind to critical information about their schools, then they could make erroneous decisions. In the complex and dynamic environment of schools, all principals need to understand effective leadership behaviors and teachers' perceptions of their behaviors. Principals must know and understand how to provide the foundation for creating an atmosphere conducive to change. Leaders must be able to correctly envision the needs of their teachers, empower them to share the vision, and enable them to create an effective school climate that creates a desire in teacher to remain in the school and profession.

Significant differences existed between a group of high attendance teachers and a group of poor or erratic attendance teachers in certain demographic factors and attitudes and perceptions regarding school climate, work satisfaction, job-related stress, and potential for burnout from the job (Rasmussen, 1996; $N=118$ ). Rasmussen explored the 
differences between two groups of teachers with different patterns of absenteeism in terms of certain demographic/personal characteristics and perceptions of school climate, job satisfactions, job-related stress, and potential burnout as a result of their work which may influence work attendance. Survey results were compared to determine differences in demographic factors, (i.e., age, gender, grade level taught, number of students in class, year of experience teaching, marital status, number of dependent children, number of miles driven to work each day, and the socio-economic status of parents in the school in which the teacher works), as well as perceptions of working conditions in the work place and school climate, job satisfactions, perceptions of job-related stress and the potential for burnout as a result of working conditions. Discriminant analysis results revealed school climate, age of respondent, job satisfaction and number of dependent children in the home distinguished the low and high attendance groups (Rasmussen, 1996). Further, Gaziel (2004) reported lower teachers' commitment to school. The principal's restrictive behavior and absentee school culture offer better explanation of variances in teacher absenteeism than any of the biographical variables.

Teachers prefer involvement in technical decisions as reported when Kappa Delta $P i$ surveyed teachers regarding their thoughts about increased involvement in school governance (Duke \& Gansneder, 1990; $N=3000$ ). Duke \& Gansneder (1990) surveyed 3,000 teachers in 100 schools regarding their thoughts about increased involvement in school governance. Teachers who were involved more frequently in either managerial $(r$ $=.50)$ or technical decisions $(r=.72)$ are more likely to think that they should be. Conversely, those who are less frequently involved in either type of decision want to be less frequently involved. While correlations are lower, teachers who are more involved in 
one type of decision making tend to be involved in the other type $(r=.19)$. And if they think that they should have high levels of involvement in one type of decision, they also think they should have similar levels of involvement in the other type of decision ( $r=$ .23 ). The survey found: (1) teachers vary in their involvement, depending on their perception of school leadership and the principal; (2) teachers may perceive that the costs of involvement outweigh the benefits; (3) involvement does not necessarily mean influence over school policies. The authors also distinguished two types of decisions: managerial (traditionally made by administrators, such as hiring teachers, allocating budgets, evaluating teachers) and technical (focused on students and instruction). The authors found that teachers prefer involvement in technical decisions (Duke \& Gansneder, 1990).

\section{School Climate in the Context of the YRE Calendar}

Educational research has linked school climate as having impact on teacher retention (August \& Waltman, 2004; Vail, 2005; $N=88$ ), but less research has linked climate in the YRE context. YRE calendar reform has positive effects on climate in schools (Boyles, 1993; $N=35$; Donahue, 1997; $N=166$; Kennedy \& Wilcher, 1998; Kneese, 2000). Teachers who have experienced the YRE school calendar in the work environment report positive impacts on teaching and personal lives. YRE teachers report increased flexibility in teaching and planning, and facilitation of educational improvements (Anderson \& Walberg, 1993; Ballinger, 1990; Kneese, 2000; White, 1987). Teachers report YRE schools have a work environment highly conducive to learning and charged with positivism. The YRE calendar helps students and teachers build better relationships (Boyles, 1993; Donahue, 1996; Kennedy \& Wilcher, 1998). 
Teachers experience less stress teaching in YRE calendar reform (Baird, 2005; Ballinger, 1987; Bradford, 1991; Nasser \& Haser, 2002; Polidor, 1996; VanderHooven, 1994). Teachers have time intervals to care of personal or business matters (Vail, 2005; Donahue, 1996). Others teachers report more time to rest, relax, or spend more time with their families through more frequent vacations.

Adams (2001) reported data analysis conducted for Phases 1 and 2 consisted of a comparison of each pair of yearly results, 1996, 1997, 1999, 2000 and 2001. Adams reported no statistically significant differences between the climate means in 1996 and 1997 but 1999 and 2000 were significantly greater than 1996 in teacher-student relationships (54.94 and 53.0 to 49.61 respectively). In 2001, the security and maintenance climate was significantly greater than in 1997 (28.12 to 24.89). In 2001, the student behavioral values were significantly greater than in 1996 (9.38 to 8.06).

\section{Summary}

The purpose of the literature review on school climate is to: (a) briefly review the theoretical foundation of school climate and critically evaluate historical attempts to measure school climate, (b) discuss important substantive implications stemming from school climate research that may advance the field, (c) present recent measurement advances, (d) highlight several methodologies that have been utilized in development of school climate instruments, and (e)explore school climate in the context of the YRE calendars. Characteristics of schools, such as the physical structure of a school building and the interactions between students and teachers, are two diverse factors that both affect and help to define the broad concept of school climate. School climate has been researched for many years and continues to be examined and redefined as a result of its 
significant influences on educational outcomes. The elements that comprise a school's climate are extensive and complex. This literature review on school climate research explored the relationship of school climate with school culture, teacher efficacy, collective efficacy, teacher job satisfaction and teacher intent to turnover. The review reported on school characteristics such as physical structures, hierarchy of the school, diversity, socio-economic status, teaching practices, symbols, urban vs rural schools, student behavior and performance, relationships of students, teachers, administrators, parents and community - the human side of the organization. When exploring the correlation of school climate and school culture, Bulach \& Peddle (2001) found a positive relationship .96 between leadership behaviors associated with instruction and the overall culture and climate of the school. Moore \& Esselman (1994) found that reading achievement performance was significantly related to teacher perception of personal efficacy $(r=.35, p=.03)$, influence in decision-making $(r=.54, p=.001)$, positive school climate $(r=.76, p=.03)$, staff collegiality $(r=.58, p=.03)$, and minimum barriers to effective teaching $(r=.71, p=.03)$. Parker (1994) found that some domains, self-efficacy and collective efficacy are related, but independent constructs. It was also found that the socioeconomic composition of a school's student body was a strong predictor of teachers' collective efficacy. When exploring school climate and teacher satisfaction, significant differences were between a group of high attendance teachers and a group of poor or erratic attendance teachers in certain demographic factors and attitudes and perceptions regarding school climate, work satisfaction, job-related stress, and potential for burnout from the job (Rasmussen, 1996). Turan (1998) found the existence of a statistically significant relationship in the following: (1) between overall 
organizational climate of schools and teacher's organizational commitment $(r=.78, p<$ $.01)$; (2) between supportive leader behavior and teachers' organizational commitment ( $r$ $=.52, p<.01) ;(3)$ between engaged teacher behavior and teachers' organizational commitment $(r=.73, p<.01)$; between direct leader behavior and teachers' organizational commitment $(r=-.27, p<.05)$; and between frustrated leader behavior and teachers' organizational commitment $(r=-.36, p<.05)$. Adams (2001) found that the security and maintenance climate of a YRE calendar was significantly greater than in 1997 (28.12 to 24.89 ). In 2001, the student behavioral values were significantly greater than in 1996 (9.38 to 8.06). The research has shown that providing a positive and supportive school climate for teachers may lead to a common culture and increases in teacher efficacy, collective efficacy, job satisfaction in the context of a YRE calendar. All of these variables may have a positive influence on teachers' intent to turnover.

\section{School Culture}

School culture can be defined as the historically transmitted patterns of meaning that include the norms, values, beliefs, ceremonies, rituals, traditions, and myths understood, maybe in varying degrees, by members of the school community (Stolp, 1994). Culture reflects the shared ideas, assumptions, values and beliefs that give an organization its identity and standard for expected behaviors (Tableman \& Herron, 2004). School culture refers more to the way teachers and other staff members work together. School culture includes everything in school surroundings that is made by human beings, consisting of tangible items as well as intangible concepts and values.

The basic idea of culture, including school culture, is that consist of shared meanings and common understanding, and that this culture is variable from school to 
school. These ideas are deeply imbedded in the organization and, to a great degree, operate unconsciously - shared understanding by teachers, staff, and students structure their responses to demands made by parents and community from the outside of the environment and from administration, school board and local/state/federal government from the inside. They are so ingrained that they are taken for granted. Based on past experience, the culture provides a template for future action - a guide to determine how things are done and to be done (Tableman \& Herron, 2004).

Culture is a result of the cultural match between an individual and an organization as determined by the degree to which the individual's personal traits fit the organizational culture, or perhaps vice versa. The elements of culture include a school's customs and traditions; historical accounts; stated and unstated understandings, habits, norms, and expectations; common meanings; and shared assumptions (Kardos \& Others, 2001). Just as culture is critical to understanding the dynamics behind any thriving community, organization, or business, the daily realities and deep structure of school life hold the key to educational success. The review of school culture research explored the relationship of the study variables of school climate, teacher efficacy, collective efficacy, teacher job satisfaction and teacher intent to turnover with school culture in the context of YRE calendars. The more understood, accepted, and cohesive the culture of a school, the better the school is able to move in concert toward ideals it holds and objectives it wishes to pursue. A lower cultural match may indicate that the individual is drained of important resources by having to continuously adjust to the workplace environment. A higher cultural match suggests the potential for a more satisfying interaction for both the 
individual and the organization (Tableman \& Herron, 2004). Some more popularly used instruments are described in APPENDIX E.

Schools are characterized by three types of professional cultures or subcultures: veteran-oriènted cultures, novice-oriented cultures, and integrated cultures (Kardos \& Others, 2001; $N=80$; scales - Riggs \& Knight, 1994; Michigan Organizational Assessment Questionnaire, Cammann et al., 1979; Mobley et al., 1978; See APPENDIX E for scale psychometrics). In veteran-oriented cultures, new teachers described norms of professional interaction determined, in large part, by the veterans, with little attention to the particular needs of beginning teachers. In novice-oriented cultures, on the other hand, new teachers described norms of professional interaction determined by novices, thus leaving them with little experienced guidance about how to teach. However, in integrated professional cultures, new teachers described being provided with sustained support and having frequent exchanges with colleagues across experience levels. Principals proved to be important in developing and maintaining integrated professional cultures where the particular needs of new teachers were both recognized and addressed (Kardos \& Others, 2001).

Morris \& Others (1995: $N=30$; Hoover-Dempsey, Bassler, Bentley \& Rempel, 1980, Brissie, 1992) reported that a weak negative cultural relationship exists between teacher self-efficacy and parental involvement and a weak positive relationship between teacher perceptions of parent efficacy and parental involvement. The researchers examined the relationship between parent efficacy, teacher efficacy, and parental involvement in nine selected schools. Questionnaires and interviews were used to solicit information from principals, teachers, and 100 randomly selected parents. Demographics 
indicated that twenty-one parents who responded lived in relatively low-income households where both parents worked outside the home. Of the fifteen teaching faculty in one school that responded, most were experienced teachers, female, and primary teachers. Findings indicated that parents and teachers agreed a culture of parental participation in volunteering at schools, telephoning teachers, and attending conferences was low. Parents did report high levels of parental involvement in helping with homework and spending time on other educational activities, but teachers did not report the same findings. The reported findings of all nine schools indicated that neither parents' self-efficacy not parents' perceptions of teacher efficacy were significantly correlated with parent involvement. Two demographic variables, family structure and family income, seemed to be moderately and consistently related to parents' and teachers' perceptions of teacher efficacy, parent efficacy and parental involvement. Teachers'selfefficacy scores were significantly and negatively correlated with several indicators of parent involvement.

Reames \& Spencer $(1998 ; N=275)$ explored the relationship between the culture of the middle school and teachers' sense of efficacy and commitment. Middle-school teachers in Georgia, representing 40 schools divided equally among rural and urban contexts, completed a four-part instrument designed to measure perceptions of schoolwork culture, organizational commitment, and teacher efficacy. School-work culture was operationalized by The School Work Culture Profile (Snyder, 1988: $N=144 ; \alpha=.95$ ) and above) which consists of four subscales: organizational planning, staff development, program development, and school assessment. Teacher efficacy was measured by Gibson and Dembo's (1984) Teacher Efficacy Scale, while teacher commitment was assessed 
with the Organizational Commitment Questionnaire (Porter, Steers, Mowday \& Boulian, $1974 ; \alpha=.90$ ). Teacher responses were analyzed to investigate the relationship between school-work culture process and structure variables on the one hand and teacher beliefs of efficacy and commitment on the other. The results revealed that all four dimensions of school-work culture were approximately equally important in explaining differences in teacher commitment and efficacy. However, these dimensions were more strongly related to the level of organizational commitment than they were to personal efficacy. General teaching efficacy was determined as not being related to the work-culture dimensions. Analysis of focus groups supported the statistical results (Reames \& Spencer, 1998). Vail (2005; $N=88)$ reports that culture affects morale enormously, but it is not always easy to read. In fact, schools may have a toxic culture to which staff is unaware. In toxic cultures, there is hostility between teachers and conflict on an ongoing basis. Rumormongers pass on negative information and talk about things that do not work rather than strategies that work. Vail (2005) states the first step to correct a toxic culture is for the principal to find a core group of staff members who believe in the school and build from there. By confronting negativity and hostility, it is possible to restore hope over time. But if negative cultures are allowed to exist for long periods, they become part of the school's reputation and are more difficult to change and retain teachers (Vail, 2005). School culture can turn from positive to toxic quickly if leaders are not vigilant. Vail uses the example of a school where staff members were very collegial; they had fun and could joke with one another. This atmosphere made it easy to share best practices and discuss important issues openly. Vail (2005) reported that a new principal came in and made decisions behind closed doors. Certain teachers gained more influence, which 
increased hostility and eroded collegiality. The staff stopped working together and began to use hidden agendas to get more resources. Some teachers requested transfers while others left the profession (Vail, 2005).

\section{School Culture in the Context of the YRE Calendar}

Teachers in YRE schools favor the YRE calendar and believe it substantially enhances the professional environment (Speck, 2002). The YRE calendar can enhance teacher professionalism in several ways (Curry, Washington \& Zsiray, 1997; Pittman \& Herzog, 1998; Shields \& Oberg, 2000; Worthen \& Zsiray, 1993). The opportunity for extended contracts and higher pay can reduce the need for teachers to moonlight in other jobs to earn extra money (Worthen \& Zsiray, 1993). Due to the frequency of breaks on the YRE calendar, teachers exhibit improved morale and motivation (Minnesota, 1999; North Carolina Insight, 1997; Polidor, 1996; Quinlan, George \& Emmett, 1987; Worthen et al., 1993). The YRE calendar fosters a culture of achievement for both students and teachers (Speck, 2002). Single-track YRE calendar schedules can potentially meet the interests of teachers who want to enhance income by working extra days and, alternatively, those who wish to keep their vacation days intact (Stenvall, 2000). In single-track schedules, teachers may choose to teach intersession classes for additional pay. Adams (2001) reported data analysis conducted for Phases 1 and 2 consisted of a comparison of each pair of yearly results, 1996, 1997, 1999, 2000 and 2001 indicated that satisfaction levels increased after the implementation of YRE. The score for teacher satisfaction for administration was significantly higher in 2001 than in 1996 (33.88 to 30.18), and there were no statistically significant differences between mean satisfaction scores in 1996 and those in 1997 in any category. In the category of compensation, the 
scores in 1996 were significantly higher than those in 2000 and 2001 (15.50 vs. 13.44 and 13.17 respectively) and the score in 1997 was significantly larger than those in 1999 , 2000 , and 2001 (17.82 vs. $14.47,13.44$ and 13.17 respectively). A second category, opportunity for advancement, showed 1997 significantly greater than those in 1999, 2000 and 2001 ( 14.50 to $11.50,10.74$ and 11.16 respectively). The teachers' satisfaction level for student responsibility and discipline was significantly greater in 2001 than in 1997 (17.16 to 14.22; Adams, 2001). Phase 2 surveys indicated stakeholders were more positive in their beliefs about teaching and learning and opinions of YRE after implementation. The results of the personal interviews reinforced the beliefs and opinions reported in Phases 1 and 2.

\section{Summary}

The purpose of the literature review on school culture is to: (a) briefly review the theoretical foundation of school culture and critically evaluate historical attempts to measure school culture, (b) discuss important substantive implications stemming from school culture research that may advance the field, (c) present recent measurement advances, (d) highlight several methodologies that have been utilized in development of school culture instruments, and (e) explore school culture in the context of YRE calendars. School culture is a subject that is receiving more and more attention in the debate over effective schools and school improvement. School culture can be described as the values, beliefs and stories of a school. School culture includes values, symbols, beliefs, and shared meanings of parents, students, teachers, and others conceived as a group or community. Culture governs what is worthy of pursuit for the school and how members should think, feel, and behave toward achieving the schools' goals. Saphier \& 
King (1985) found significant correlations were found between School Culture Survey subscales (teacher professionalism and administrator professional treatment of teachers $(r$ $=.71, p<.001)$, teacher collaboration and administrator professional treatment of teachers $(r=.51, p<.001)$, teacher professionalism and teacher collaboration $(r=.61, p$ $<.001)$ and teacher efficacy, empowerment, and conceptual level. Reames and Spenser (1998) revealed that all four dimensions of school-work culture (organizational planning, staff development, program development, and school assessment) were approximately equally important in explaining differences in teacher commitment and efficacy. Vail (2005) reports that culture affects morale enormously, but it is not always easy to read. In fact, schools may have a toxic culture to which staff is unaware. In toxic cultures, there is hostility between teachers and conflict on an ongoing basis leading to intent to lurnover. Washington \& Zsiray (1997), Pittman \& Herzog (1998), Shields \& Oberg (2000), and Worthen \& Zsiray (1993) report that the YRE calendar can enhance teacher professionalism.

\section{Teacher Efficacy}

Teacher efficacy, usually defined as teachers' expectations that they will be able to perform the actions that lead to student learning, is both a contributor to and consequence of school reform (Ross \& Others, $1999 ; N=359$ ). In addition, the empirical foundation for this claim rests on studies that treat teacher efficacy as a stable, unitary trait, despite evidence that within teachers it fluctuates over time and varies with teacher tasks. Wyatt (1998) reported that the most effective teachers produce as much as six times the learning gains as the least effective teachers. Sanders $(2000)$ reports the difference in teacher effectiveness is the single largest factor affecting academic growth 
of populations of students. A student who is taught by an ineffective teacher for two years in a row can never recover the learning lost during those years (Sanders, 2000). Students who have several effective teachers in a row make dramatic achievement gains, while those who have even two ineffective teachers in a row lose significant ground (Sack, 1999). Researchers have used a variety of teacher efficacy instruments to measure efficacy. Some more popularly used instruments are described in APPENDIX F. Soodak \& Podell (1996; $N=310$; Teacher Efficacy Scale; See APPENDIX F for psychometrics) explored the dimensions of teacher efficacy. Results indicated teacher efficacy comprises three uncorrelated factors (personal efficacy, outcome efficacy, and teaching efficacy), underscoring the need for ongoing exploration of the dimensions of this construct. People "enter work organizations with expectations and values, and if these expectations and values are met, they will likely remain a member of the organization" (Dee, 2002, p.4; $N=149$ ). Teachers with high efficacy attitudes tended to maintain high academic standards, concentrate on academic instruction, monitor classroom environment, develop a warm, supportive classroom environment, and their students had higher achievement test scores than did students of teachers with low efficacy attitudes (Smith \& Rowley, 2005; National Center for Educational Statistics, Schools and Staffing Survey (SASS); Ashton \& Webb, 1983). Significant relationships among teacher efficacy, student-teacher interaction, and student achievement were found. Current conditions in the school - isolation, uncertainty, powerlessness, and lack of economic rewards and social recognition - appeared to be factors that contribute to a low sense of efficacy in teachers. School organizational structures of teaming, multi-age 
grouping, and collegial decision-making among teachers appeared to be school factors that may increase teacher efficacy (Ashton \& Webb, 1983).

Professional efficacy is described as the beliefs teachers have about the positive effects they can have on student learning and that schools can make a difference regardless of factors of home and community (Bandura, 1977). Professional efficacy supports the belief that all children can learn and, based on the Kentucky Education Reform Act of 1990, learn at high levels. Hoy \& Woolfolk (1990) state that teachers' sense of efficacy includes two independent dimensions: personal efficacy and professional efficacy. The first dimension is teachers' assessment of their own competence or their personal efficacy. Teachers with high personal efficacy have the ability to teach or to know how to adjust their teaching to reach all kids. On the other hand, teachers with low personal efficacy doubt their abilities to reach all kids and tend to have higher intent to turnover. Hoy \& Woolfolk's second dimension is professional efficacy which reflects teachers' expectation that teaching and schools can influence student learning. Teachers with high professional efficacy believe that students are capable of learning. Teachers with low professional efficacy believe that some children can not learn and that home and community influences are too great to overcome. Teachers with low professional efficacy tend to have a high intent to turnover (Hoy \& Woofolk, 1990). Teachers' sense of efficacy, the extent to which teachers believe that they have the capacity to affect pupil performance, is related to both teaching behaviors and pupil performances (Pigge \& Marso, 1993).

Teacher efficacy, both personal and teaching, is influenced by the context of the workplace (Moore \& Esselman, 1994; $N=1,500$ ). This study hypothesizes that a sense 
of personal and teaching efficacy can be explained, in part, by a historical pattern of student achievement performance and workplace context. To measure perceptions of efficacy, power, and school climate, a questionnaire was completed by approximately 1,500 elementary school teachers in the spring terms of 1991, 1992, and 1993.

Achievement scores on the Iowa Tests of Basic Skills for 5 years preceding the first survey determined students' historical achievement performance. Findings indicated that context was an important influence on teaching efficacy; that a positive school atmosphere (focused on instruction), the reduction of barriers to effective teaching, and classroom-based decision-making each contributed to teachers' sense of teaching efficacy; and that schools with historically poor achievement tended to have teachers who reported a poorer image of school atmosphere which contributed to poorer perceptions of teaching effectiveness. Results suggest opportunities for improving the self-view of teachers and their profession. Specific recommendations include improvement of the instructional focus and climate of schools and provision of greater opportunity for teachers to participate and be influential in instructional and curricular decisions.

Collective efficacy has a moderating effect on teachers' self-efficacy (Hongyun et al., 2005; $N=1299)$. The important effects of collective efficacy as a school context characteristic variable were examined, including both as a predictor to explain the teachers' mean difference among schools and as a moderator moderating the relations of self-efficacy and teachers' characteristic variables, such as teachers' job satisfaction, work devotion, internal motivation and the satisfaction of colleague relationship and demission tendency. Using the Hierarchical Linear Model (HLM), study results revealed that: (1) Teachers' self-efficacy significantly predicted teachers' job satisfaction, work devotion, 
internal motivation and the satisfaction of colleague relationship and teacher turnover tendency; however, there were school level variations among these effects; (2) The higher the school's collective efficacy, the school means of teachers' job satisfaction, work devotion, internal motivation and the satisfaction of colleague relationship were, the lower teacher turnover tendency was; (3) The relationships between self-efficacy, other teachers' characteristics and teachers in higher collective efficacy schools was moderated by school-level collective efficacy. In addition, teachers' self efficacy was more positively related to teachers' job satisfaction, work devotion, internal motivation and the satisfaction of colleagues than did those in lower collective efficacy schools, but more negatively related to teacher turnover tendency (Hongyun et al., 2005).

Gresham (2002) explored the relationships between thirty-nine schools' fifth grade teachers' perceptions of their own teaching efficacy, teacher job satisfaction, student socioeconomic status and the academic success of students. The researcher used The Teacher Efficacy Scale (Gibson \& Dembo, 1984, the Teacher Job Satisfaction Questionnaire (Lester, 1987; See APPENDIX G for scale psychometrics) and student achievement scores on state assessment. Results indicated correlations between teachers' perception of their self-efficacy, both personal and teaching efficacy, and job satisfaction were found to be not significant at the $p=.05$ alpha level, $r=.02$. The correlations between teacher job satisfaction and academic success were found to be not significant at the $p=.05$ alpha level, $r=.22, .22, .21, .26$, respectively for English, math, science and social studies. The correlations between teacher self-efficacy perceptions, both personal teaching efficacy and teaching efficacy, and the socioeconomic status of a school's students were found to not be significant at the $p=.05, r=.08$ and .12 respectively. 
Correlations between job satisfaction and socioeconomic status of students showed no significance $(r=-.20)$.

Chambers (2003; $N=55$; Attitudes and Beliefs on Classroom Control Inventory, Martin, Yin \& Baldwin, 1998 and the Teacher Efficacy Scale, Gibson \& Dembo, 1984; See APPENDIX F for psychometrics) reports the length of a teacher education program will affect student teachers' self-efficacy and classroom management beliefs. Also noted was whether there were differences between students who had been in one semester versus two semesters of student teaching in regard to classroom management and selfefficacy beliefs. During the spring and fall semesters, Chambers had secondary teacher education students from a mid-sized Texas university to participate in the study. Participants included twenty-eight who were completing the traditional two-semester student teaching program and twenty-seven who were completing the one-semester program. Students in the one-semester program had the same experiences as those in the two-semester program through an intensified semester. Near the end of the student teaching semesters, teacher education students completed two questionnaires, the Attitudes and Beliefs on Classroom Control Inventory (ABCC) and the Teacher Efficacy Scale. Three subscale scores in the ABCC, instructional management, people management, and behavioral management, were examined as predictors of teacher selfefficacy as indicated on the TES. The researchers used a simple regression procedure and found that only one $\mathrm{ABCC}$ subscale, instructional management, would enter the regression equation and accounted for sixteen percent $(16 \%)$ of the variance in teacher self-efficacy $\left(r^{2}=.16\right)$ a moderate predictor. Data analysis indicated that there were no 
differences in the belief systems of students who participated in the two-semester versus one-semester programs (Chambers, 2003).

Hoy (2000; $N=27$ and 28 in two cohorts) reports that the greater the support and buffering during student teaching experience, the greater the increase in efficacy and the fewer the sick days. The study used a longitudinal investigation that assessed the efficacy of prospective and novice teachers at the beginning of their preparation program, the end of student teaching, and after their first year of employment. Students were randomly assigned to the cohorts after being accepted in the teacher preparation program. Students completed three instruments to assess efficacy: Woolfolk and Hoy's Teacher Efficacy Scale, Bandura's Teacher Self-Efficacy Scale, and Teaching Confidence Scale. T-tests for paired samples indicated that the changes from the beginning of the program to the end of student teaching (Phase 1 to Phase 2) represented significant increases in efficacy. From the end of student teaching to the end of the first year of teaching, the decreases in the Bandura, general teaching efficacy (GTE), and personal teaching efficacy (PTE) scales were significant, but the slight decrease in the Teaching Confidence Scale was not significant. From entry into the program to the end of the first year of teaching (Phrase 1 - Phase 3), the increases in efficacy indicated by the PTE and Teaching Confidence Scale measures were significant. Some general conclusions from the study report efficacy rose during teacher preparation, but it fell with the actual experience as a teacher. As teacher efficacy increased, sick days decreased. The samples probably had more support and buffering during their student teaching experience and when this support is withdrawn, efficacy falls. Confident new teachers gave higher ratings to the adequacy of support they 
had received than those who ended their first year with a shakier sense of their own competence (Joffres \& Haughney, 2001).

Participants in the Joffres \& Haughney (2001) study expressed that they felt unprepared to teach up to the completion of their degree, which fostered low feelings of efficacy. Depending on their circumstances, the teachers felt inadequately trained to deal with the many different curricula, classroom behavior management, large classes, age specific children, children's diverse learning styles, and/or the preparation and presentation of lessons (Joffres \& Haughney, 2001; $N=14$ elementary teachers; case study on teacher commitment; semi-structured interviews). Participants' feelings of inadequacy increased with administrators' failure to provide teachers with "constructive, regular, and specific" performance feedback, to promote cultural norms such as collaboration, mentorship, and professional development fostering information exchange and teachers' learning, and/or to implement and enforce school-wide behavioral policies fostering orderly class environments, a prerequisite for student learning. Teachers felt unreasonable workloads also precipitated the informants' feeling of failure. Role overload was fostered by teachers' already low feelings of adequacy, too high expectations for self, classes with students at very different levels or disorderly classes, felt helplessness and/or school administrators' unreasonable demands or expectations. Demands were deemed "unrealistic" when teachers did not have enough time/resources/training to adequately facilitate student learning and/or via comparisons with previous assignments and/or actual assignments of comparable others. Teachers felt a lack of time, tired (caused by the extra hours that teachers put in to try and keep up with their workload and their sense of inefficacy), the guilt resulting from the teachers' 
perceived inability to address the students' needs, and the informants' concerns for their professional reputation caused many teachers to decrease their contacts with their peers and the children's parents, two potential sources of social rewards (Joffres \& Haughney, 2001). Participants reported they temporarily sacrificed their leisure time and familial activities to their work, which increased their anger and feelings of guilt toward their family. However, teachers report, in spite of these sacrifices, they were often unable to catch up with their work and developed feelings of panic, anxiety, and /or symptoms of ill health. Participants also experienced a great deal of dissatisfaction when they become emotionally and intellectually unable to experience teaching as challenging, that is, when they experienced a growing sense of boredom. Boredom and the resulting sense of meaninglessness it facilitated among the informants was often bolstered by a combination of factors, such as little or no professional growth, often increased by the schools' lack of emphasis on collaboration and innovation, and felt inadequate professional development), a sense of task routinization, a lack of upward and lateral mobility, and/or a growing disinterest in students. Teachers also felt very unsuccessful when they realized that valued goals (e.g., a permanent contract, a principalship would probably never be met, or would not be met within the time limits that they had initially hoped (Joffres \& Haughney, 2001)..

\section{Teacher Efficacy in the Context of the YRE Calendar}

Teachers in YRE calendar programs generally believe that the quality of instruction is better than the quality of instruction in traditional agrarian school calendar programs due to the continuity of instruction (Kneese, 2000; Quinlan, George \& Emmett, 1987; Shields \& Oberg, 2000; VanderHooven, 1994). Students forget less over the 
shorter vacations and teachers review less previously learned material and concepts. Intersession intervals give teachers time to think and plan for their next units and that the units were more interesting for students (Kneese, 2000). The organization of instructional time allows teachers to be more reflective practitioners because they are able to plan at regular intervals during the academic year when planning is needed the most (Cooper, 1996; Shields \& Oberg., 2000). YRE teachers find planning more efficient and productive when planning curriculum for shorter blocks of time and feel the YRE calendar provides ample time segments for instruction (Haser \& Nasser, 2005; Nasser \& Haser, 2002; National Commission on Time and Learning, 1993). Teachers report increased effectiveness in the use of time (Bradford, 1993; Dossett \& Munoz, 2000; Elsberry, 1993; Warrick-Harris, 1995; Wildavsky, 1997). During interviews, Donahue (1997) report that YRE teachers have a high sense of efficacy and tend to be more open to new ideas, more willing to experiment with new methods to better meet the needs of their students, and more committed to teaching. They persist when things do not go smoothly and are more resilient in the face of setbacks (Donahue, 1997). YRE teachers report that they tend to be less critical of students who make errors and to work longer with a student who is struggling (Boyles, 1993).

Boyles (1993) reported that when YRE teachers and traditional calendar matched pair schools were asked about satisfaction with teaching in the past, present and expected future in their current schools, YRE teachers were more positive about their teaching in the present and expected future with $86 \%$ rating their satisfaction high with the present and expected future while traditional calendar teachers were more positive about their satisfaction with $71 \%$ rating high satisfaction with their past teaching and $38 \%$ and $71 \%$ 
for satisfaction with present and expected future teaching, respectively. When asked about satisfaction with their teaching effectiveness, YRE teachers were more positive about their teaching effectiveness in the past, present and expected future with 50\% rating their satisfaction high with the past, $72 \%$ for the present and $86 \%$ for the expected future while traditional calendar teachers were less positive about their teaching effectiveness with $42 \%$ rating high satisfaction with their past teaching effectiveness and $46 \%$ and $75 \%$ for satisfaction with present and expected future teaching effectiveness, respectively.

\section{Summary}

The purpose of the literature review on teacher efficacy is to: (a) briefly review the theoretical foundation of teacher efficacy and critically evaluate historical attempts to measure teacher efficacy, (b) discuss important substantive implications stemming from efficacy research that may advance the field, (c) present recent measurement advances, (d) highlight several methodologies that have been underutilized in development of teacher efficacy instruments, and (e) explore teacher efficacy in the context of the YRE calendars. Teacher efficacy examines the factors that contribute to the confidence teachers have to successfully achieve their goals related to classroom instruction, reflective teaching, classroom management, engaging students, motivating students and other stakeholders in the educational process, as well as other related areas that contribute to the growing understanding of what influences teacher efficacy. This literature review on school teacher efficacy research explored the relationship of teacher efficacy with school climate, school culture, collective efficacy, teacher job satisfaction and teacher intent to turnover in the context of YRE calendars. Tucker (2003) reported teacher efficacy and school climate correlation results that indicated the relationships between 
teacher morale and job satisfaction $(r=.16, p=.0001)$; morale and robustness $(r=.44, p$ $=.0001)$; morale and general teaching $(r=.19, p=.0001)$; morale and personal efficacy $(r=.17, p=.0001)$; satisfaction and both teaching $(r=.14, p=.0001)$; satisfaction and personal $(r=.17, p=.0001)$; total efficacy and morale $(r=.23, p=.0001)$ and total efficacy and satisfaction $(r=.22, p=.0001)$. When exploring teacher efficacy and school culture, Morris \& Others (1995) found that two demographic variables, family structure and family income, seemed to be moderately and consistently related to parents' and teachers' perceptions of teacher efficacy, parent efficacy and parental involvement. Chambers (2003) reported found that instructional management accounted for sixteen percent $(16 \%)$ of the variance in teacher self-efficacy $\left(r^{2}=.16\right)$ a moderate predictor. The Southeast Center of Teaching Quality (2004) found results indicating teachers' working conditions are significant and strong predictors of student achievement; Leadership is critical to improving working conditions, but principals and teachers perceive these conditions very differently; Teachers are generally satisfied with most aspects of their working conditions, and since 2002, their views about them are improving across North Carolina; and Teachers, regardless of their background and experience, view working conditions similarly. Further, Weiss (1999) found that teachers highly involved in their work attributed their decision to stay in teaching more the result of supportive workplace conditions than to pay. Finally, Boyles (1993) reported that when YRE teachers and traditional calendar matched pair schools were asked about satisfaction with teaching in the past, present and expected future in their current schools, YRE teachers were more positive about their teaching in the past, present and expected future. 


\section{Collective Efficacy}

Collective efficacy refers to an individual's assessments of his or her group's competency and likelihood for success. Collective efficacy has been measured as an aggregate of individual members' self-efficacy or as an agreed-upon amount derived from group discussions (Zellars \& Others, 2001; $N=380$ nurses; Collective Efficacy Scale, Riggs \& Knight (1992; See APPENDIX G for scale psychometrics ). Perceived collective efficacy was negatively correlated with role conflict $(p<.01)$ and intent to turnover $(p<.01)$, and positively correlated with job satisfaction $(p<.01)$. The researchers used hierarchical moderated regression to test Hypothesis 2 by entering the interaction term (role conflict x perceived collective efficacy) in step 3. Results of the regression analysis indicated the overall models for job satisfaction $(F=15.19, p<.01)$, exhaustion $(F=15.77, p<.01)$, and intent to turnover $(F=15.38, p<.01)$ were significant and explained $35-36 \%$ of the variance in each outcome. Age and gender failed to significantly predict any of the work outcomes. As expected, self-efficacy positively predicted job satisfaction $(p<.05)$ and negatively predicted exhaustion $(p<.01)$. Direct effects were also found for role conflict on job satisfaction $(p<.01)$, exhaustion $(p<$ .01$.$) , and intent to turnover (p<.01)$. Consistent with previous studies, perceived collective efficacy directly and positively predicted job satisfaction $(p<.01)$ and negatively predicted intent to turnover $(p<.01)$, explaining an additional $3-4 \%$ of the variance in these outcomes. However, collective efficacy did not explain additional variance in exhaustion beyond self-efficacy scores. Perceived collective efficacy will moderate the relationship between role conflict and job satisfaction, exhaustion, and intent to turnover. Specifically, perceptions of greater collective efficacy will reduce the 
negative relationship between role conflict and job satisfaction and reduce the positive relationship between role conflict and emotional exhaustion and intent to turnover. Recent research in the area of collective efficacy has utilized several scales. The more popularly used scales are described below in APPENDIX G. Some of the more wellknow collective efficacy scales include: Collective Teacher Efficacy Scale (Goddard, 2002a; correlation between personal teaching efficacy and collective teacher efficacy $(r=$ $.54, p<.01)$, and between faculty trust in colleagues and collective teacher efficacy $(r=$ $.62, p<.01$ ); Collective Teacher Self-Efficacy Scale (Schwarzer, Schmitz \& Daytner, 1999; $N=300$ ), and Sense of Powerlessness Scale (Zielinski \& Hoy, 1983; APPENDIX G for scale psychometrics).

Failure to influence students' learning resulted from inadequate training and/or experience, role conflict and role overload, disorderly classroom environments, and normlessness regarding the school behavioral policies or little support from school staff (particularly the principal) to enforce existing discipline policies. In addition, teachers also placed influence on principals' failure to develop cultural norms facilitating teachers' learning (Joffres \& Haughney, 2001; $N=14$ ). The researchers explored factors influencing teachers' feelings of collective efficacy. When the teachers in the study reported they could not influence children's learning, they also reported a feeling of being profoundly unsuccessful to the point of uselessness. When teachers were asked, in semistructured interviews, to relate what they felt attributed their failure to influence students' learning, teachers reported a combination of factors: inadequate training and/or experience, role conflict and role overload, disorderly classroom environments, normlessness regarding the school behavioral policies or little support from school staff 
(particularly the principal) to enforce existing discipline policies, principals' failure to develop cultural norms facilitating teachers' learning (e.g., collaboration, mentorship, professional development), minorities' relentless and angry interferences (e.g., parents opposing the implementation of a program), and/or personal characteristics such as a poor self-concept or too high expectations for self (Ray, 2005).

Collective efficacy is the extension of Bandura's (1977) self-efficacy concept to groups (Bandura, 1982, 1986). Self-efficacy refers to judgments people make about their personal or individual competency. Collective efficacy concerns judgments that people make about a group's level of competency (Parker, 1994). Parker explored elementary school teachers' collective efficacy and self-efficacy. The academic domain is well suited to the study of collective efficacy because the organizational units (schools) all perform the same function (education). The findings supported the hypothesis that, in at least some domains, self-efficacy and collective efficacy are related, but independent constructs. It was also found that the socioeconomic composition of a school's student body was a strong predictor of teachers' collective efficacy. Although teachers' collective efficacy was associated with school level achievement, this relationship was no longer significant when prior achievement levels were controlled. Correlations between teachers' self-reported years of experience in teaching, years of experience in the school, gender, and perceptions about collective efficacy were also calculated. The correlation between collective efficacy and gender was small but statistically significant $(r=.12, p<$ .001 ), suggesting that females perceive higher levels of collective efficacy than males. The correlations between perceived collective efficacy and teaching experience, however, was negligible $(r=.05, p<.01)$, suggesting that individuals who remain in the profession 
longer are no more likely than novice teachers to perceive their colleagues as effective. Additionally, teachers who remained in the same school for a long period of time were no more likely than new teachers to perceive their colleagues (in that school) as effective ( $r$ $=.03, p<.01)$. Teachers' perceptions of collective efficacy and their perceptions of professional learning community readiness are moderately related. This suggests that teachers who perceive their school to be characterized by shared leadership, focused vision, collaborative work, shared observation, and supportive conditions also perceive their colleagues to be effective in bringing about student learning.

Mawhinney et al. (2005; $N=2,448)$ attempted to increase knowledge about select relationships of teachers' perceptions of collective efficacy and conditions for professional learning communities at the school level. Results of the subscales indicated the following: principal's facilitative leadership (.86); shared visions for improvement (.88); collective creativity and learning (.88); classroom observations and feedback (.82); school conditions and capabilities (.83); and collective efficacy beliefs (.75). In general, elementary teachers appear to perceive higher collective efficacy and a more positive school culture for professional learning communities to develop than do middle and high school teachers. Furthermore, results indicated perceptions generally tend to be more diverse among secondary compared to elementary school teachers. Mawhinney et al. (2005) reported that the correlation between collective efficacy and gender was small but statistically significant $(r=.12, p<.001)$, suggesting that females perceive higher levels of collective efficacy than males. The correlations between perceived collective efficacy and teaching experience, however, was negligible $(r=.05, p<.01)$, suggesting that individuals who remain in the profession longer are no more likely than novice teachers 
to perceive their colleagues as effective. However, these dimensions were more strongly related to the level of organizational commitment than they were to personal efficacy. General teaching efficacy was determined as not being related to the work-culture dimensions.

Goddard et al.(2004) examined the relationship between collective efficacy and high school student achievement in ninety-six schools a state with an accountability system heavily focused on achievement, measured by mandatory assessment in multiple content areas (e.g., like Kentucky). The study used the social cognitive theory; the researchers developed a theoretical model developed linking school context and collective efficacy to difference among schools in $12^{\text {th }}$ grade achievement. To test the fit of the model to data drawn from the students and teachers in the selected ninety-six state high schools, the researchers used a structural equation. The results indicated collective efficacy was positively influenced by past mastery experience and negatively related to school socioeconomic disadvantage (Gresham, 2002).

Collective teacher efficacy has significant affect on student achievement (Goddard et al., 2000). In the study, $N=452$ teachers at 47 urban elementary schools completed the twenty-one item CTES; 7,016 of their 2nd, 3rd, and 5th grade students completed mathematics and reading achievement. All items loaded strongly on a single factor and explained $57-9 \%$ of the variance. The strength of correlation between these factors $(r=.75, p<.001)$ provided further evidence that collective teacher efficacy is the common unobserved factor operationalized by the revised collective efficacy scale. More over, the collective teacher efficacy measure directly assesses perceptions of both perceived competence and task whereas the personal teacher efficacy measure includes 
on items about competence. As predicted, there was a moderate and positive ( $r=35, p<$ $.01)$ correlation between personal teacher efficacy aggregated at the school level and collective teacher efficacy. Results indicated that the CTES demonstrated adequate validity and reliability. The theoretical elements of collective teacher efficacy, group competence, and task analysis were highly related in schools. Collective teacher efficacy was positively associated with differences between schools in student level achievement in both reading and mathematics. Collective teacher efficacy perceptions were predictive of student achievement (Goddard et al., 2000).

Teacher collaboration has a causal effect on trust and collective teacher efficacy (Dale, 2005; $N=545$ ). The purpose of this study was to examine the causal relationships between collaboration, trust, and collective teacher efficacy. Participants in the study were teachers selected from 79 schools throughout the northeastern quadrant of Oklahoma. School level data was obtained from an $N=545$ teachers and $N=79$ administrators. A theoretical causal model was developed consisting of eight variablessocioeconomic status, teacher trust of teacher, teacher trust of principal, teacher-teacher collaboration, teacher principal collaboration, prior academic skill, school level, and collective teacher efficacy-to test the direct and indirect relationships among the variables. Teacher-teacher collaboration was found to be a significant predictor of both teacher-teacher trust and collective teacher efficacy. Prior academic skill and teacherteacher trust were found to be significant predictors of collective teacher efficacy. Teacher-principal collaboration was not found to be a significant predictor of either teacher-principal trust or collective teacher efficacy. Teacher-principal trust was also not a significant predictor of collective teacher efficacy. Findings from this research highlight 
the importance of collaboration in building trusting relationships and in raising the level of collective efficacy within a school. Dale (2005) highlighted the importance of collaboration in building trusting relationships and in raising the level of collective efficacy within a school.

Teacher efficacy, collective efficacy, and collaborative climate have relationships and are statistically significant regarding school effectiveness (Mackenzie, 2001; $N=$ 384; Collective Efficacy Scale, (See APPENDIX G for scale psychometrics). $N=792$ teachers at 21 Maine high schools received the Teacher Survey. Three hundred eightyfour teachers completed and returned the survey within the month, a response rate of $48 \%$. The researcher found a significant correlation between teacher efficacy and collective efficacy $(r=.44, p<.01, N=384)$ at the teacher level. Another way of thinking about the strength of the teacher efficacy /collective efficacy relationship is to look at the coefficient of determination of the relationship $\left(r^{2}=.19\right)$. It indicates roughly $20 \%$ of the variation in perceptions regarding collective efficacy and teacher efficacy was shared or common variation (Mackenzie, 2001).

Collective efficacy is related to collaborative climate, specifically, shared goals and collegiality (Guskey, 1998) and a potentially powerful influence of organizational variables such as support, resources, peer relations, and collaboration. The policy implications of collective efficacy for school leaders can be a powerful concept for heightening awareness of a school's capacity for organizing and implementing effective actions to meet goals because collective efficacy is strongly associated with teachers' having shared goals and the dynamics of collaborative climate are clearly connected to teachers' assessments of their school's collective efficacy (Guskey, 1998). Reformers 
contend a collaborative climate is conducive to effective schools. Collective efficacy is logically connected to collaborative climate and has the potential to enhance understanding of the psychosocial aspects of schools as organizations. After analysis by examining descriptive statistics and correlations among variables at teacher and school levels and within schools, findings at the teacher level indicated a moderate correlation between collective efficacy and teacher efficacy. The variables of collegiality and shared goals were strongly correlated, but both were moderately correlated with collaborative work. Further, collective efficacy was moderately correlated with collegiality and shared goals but less so with collaborative work. School level correlations were strong except for a weak correlation between collective efficacy and collaborative work. Collective efficacy and collaborative climate were school level phenomena, so teachers at the schools had more common perceptions of these variables than did individual teachers. The individual nature of teacher efficacy was reinforced by the negligible associations between teacher efficacy and any of the collaborative climate variables at both the teacher and school levels. The within school analyses proved problematic for the researcher because of the small number of respondents and lack of linear relationships in some instances. The researcher concludes that this study of high school teachers supported the notion that teacher and collective efficacy are related concepts but that they function differently and have different correlates (Guskey, 1998).

Goal consensus/vision has a relationship with individual teacher efficacy and collective teacher efficacy (Kurz \& Knight, 2004: $N=113$; Teacher Efficacy Scale, Gibson \& Dembo, 1984; See APPENDIX G for scale psychometrics). This study explored the relationships among individual teacher efficacy, collective teacher efficacy, 
and goal consensus/vision. Participants included 113 teachers of a high school located in the southwestern USA. Teachers completed three surveys that would measure the variables under study. Correlational and regression analyses to examine the relationships among individual teacher efficacy, collective teacher efficacy, and goal consensus/vision were performed. Findings indicated that collective teacher efficacy correlated with all of the other variables examined, but was most highly correlated with goal consensus/vision. In addition, individual teacher efficacy, while related to collective teacher efficacy, was not found to be related to goal consensus/vision. In conclusion, the researchers suggest that, because individual teacher efficacy, collective teacher efficacy and goal consensus/vision are related, changing one could have an impact on the others (Kurz \& Knight, 20(14).

The socio-economic composition of a school's student body is a strong predictor of teachers' collective efficacy (Parker, 1994). Schools with historically poor achievement tended to have teachers who reported a poorer image of school atmosphere which contributed to poorer perceptions of teaching effectiveness (Moore \& Esselman, 1994). This study contributes to the theory and measurement of collective efficacy and to an understanding of its relationship to self-efficacy and performance. Elementary school teachers' collective- and self-efficacy were measured. The researcher concluded that the academic domain is well suited to the study of collective-efficacy because the organizational units (schools) all perform the same function (education). The results supported the hypothesis that, in at least some domains, self-efficacy and collectiveefficacy are related but independent constructs. As hypothesized, it was also found that the socioeconomic composition of a school's student body was a strong predictor of 
teachers' collective-efficacy. Although teachers' collective-efficacy was associated with school level achievement, this relationship was no longer significant when prior achievement levels were controlled. It was suggested that future research should examine individual behaviors, attitudes, and beliefs that might serve as mediators between efficacy and performance. It was also suggested that the relationship between collective efficacy and organizational culture should be explored. After accounting for the influence of several aspects of school context, the results showed that collective efficacy remained a significant positive predictor of student performance across the mandated content areas (Parker, 1994).

A positive relationship exists between teacher and collective efficacy and student achievement in urban schools (Goddard \& Goddard, 2001; $N=438$; Collective Teacher Efficacy Scale, See APPENDIX G for scale psychometrics). Teacher responses to the teacher efficacy scale items were submitted to a principal axis factor analysis. One factor was extracted with an eigenvalue of 2.106 explaining $42.12 \%$ of the variance. Factor loadings ranged from .62 to .70 . The alpha coefficient of reliability for the teacher efficacy scale was .79. The teacher efficacy score for each teacher was constructed as the mean of the teacher's responses to all items in the teacher efficacy scale. Researchers developed a model and an operational measure of collective teacher efficacy. An unconditional analysis (one-way ANOVA with random effects) indicated significant variation among schools in teacher efficacy. Specifically, variation among the school means for teacher efficacy $\left(\operatorname{Var}\left(\mathrm{B}_{0 \mathrm{j}}\right)\right)$ was $.05\left(r^{2}=67.33, d f=46, p<.05\right)$. This finding confirmed that teacher efficacy does indeed vary systematically with school characteristics. Given that teacher efficacy varied significantly among schools, we 
continued our analysis by building a school-level model to explain that variation. Results showed collective teacher efficacy to be positively associated with student-level achievement in reading and mathematics Results indicated that collective efficacy predicted variation in teacher efficacy above and beyond the variance explained by the number of school contextual factors, including socioeconomic status and student achievement. The theoretical elements of collective teacher efficacy, group competence, and task analysis were highly related in schools. Collective teacher efficacy was positively associated with differences between schools in student-level achievement in both reading and mathematics (Gresham, 2002; Goddard, Hoy \& Hoy, 2000, Goddard et al., 2001 \& Goddard, 2001).

Hongyun, Lei \& Oingmao $(2005 ; N=1299)$ explored the effects of collective efficacy as a school context characteristic variable, including both as a predictor to explain the teachers' mean difference among schools and as a moderator moderating the relations of self-efficacy and teachers' characteristic variables, such as teachers' job satisfaction, work devotion, internal motivation and the satisfaction of colleague relationship. Based on survey data collected from 1299 teachers representing 28 elementary schools, the important effects of collective efficacy as a school context characteristic variable were examined, including both as a predictor to explain the teachers' mean difference among schools and as a moderator moderating the relations of self-efficacy and teachers' characteristic variables, such as teachers' job satisfaction, work devotion, internal motivation and the satisfaction of colleague relationship and demission tendency. Hierarchical Linear Model (HLM) results revealed that (1) teachers' selfefficacy significantly predicted teachers' job satisfaction, work devotion, internal 
motivation and the satisfaction of colleague relationship and demission tendency, but there were school level variations among these effects; (2) the higher the school's collective efficacy, the school means of teachers' job satisfaction, work devotion, internal motivation and the satisfaction of colleague relationship were higher, and demission tendency was lower: (3) School-level collective efficacy moderated the relationships between self-efficacy and other teachers' characteristics, teachers in higher collective efficacy schools, the teachers' self efficacy was more positively related to teachers' job satisfaction, work devotion, internal motivation and the satisfaction of colleagues than did those in lower collective efficacy schools, but more negatively related to demission tendency (Hongyun, Lei \& Oingmao, 2005).

\section{Collective Efficacy in the Context of the YRE Calendar}

When YRE teachers took the Elements of Quality survey covering such topics as school management, community confidence in school, and organization of school, YRE teachers scored higher than teachers on a traditional calendar in all areas, though not at significant levels (Costa, 1987). Another study asking about the ease of scheduling personal and family activities showed that YRE teachers expressed significantly higher satisfaction in this area than traditional calendar teachers (Elsberry, 1992). The Purdue Teacher Opinionnaire was utilized in one study that found no difference in staff morale between teachers on YRE and traditional schedules (Nygaard, 1974). Finally, when queried about school climate and collective effectiveness, YRE staff had a more positive attitude than did those on a traditional calendar in fifteen out of eighteen survey questions, although no significance tests were conducted (Prohm and Baenen, 1996). 


\section{Summary}

The purpose of the literature review on collective efficacy is to: (a) briefly review the theoretical foundation of collective efficacy and critically evaluate historical attempts to measure collective efficacy, (b) discuss important substantive implications stemming from collective efficacy research that may advance the field, (c) present recent measurement advances, (d) highlight several methodologies that have been underutilized in development of collective efficacy instruments, and (e) explore collective efficacy in the context of the YRE calendars. Collective efficacy examines the factors that contribute to the collective confidence teachers have to successfully achieve their goals related to school-wide instruction, reflective teaching, classroom management, engaging students, motivating students and other stakeholders in the educational process, as well as other related areas that contribute to the growing understanding of what influences collective efficacy. This literature review on school collective efficacy research explored the relationship of collective efficacy with school climate, school culture, teacher efficacy, teacher job satisfaction and teacher intent to turnover in the context of YRE calendars. Mackenzie (2001) found a significant correlation between teacher efficacy and collective efficacy $(r=.44, p<.01, N=384)$ at the teacher level. Another way of thinking about the strength of the teacher efficacy/collective efficacy relationship is to look at the coefficient of determination of the relationship $\left(r^{2}=.19\right)$. Teacher efficacy accounts for roughly $20 \%$ of the variation in perceptions regarding collective efficacy. Kurz \& Knight (2004) found that collective teacher efficacy correlated with all of the other variables examined (individual teacher efficacy, collective teacher efficacy, and goal consensus/vision), but was most highly correlated with goal consensus/vision. Goddard \& 
Goddard (2001) indicated significant variation among schools in teacher efficacy. Specifically, variation among the school means for teacher efficacy $\left(\operatorname{Var}\left(\mathrm{B}_{0_{\mathrm{j}}}\right)\right)$ was .05 $\left(r^{2}=67.33, d f=46, p<.05\right)$. This finding confirmed that teacher efficacy does indeed vary systematically with school characteristics. Gresham (2002) found results that indicated correlations between teachers' perception of their self-efficacy, both personal and teaching efficacy, and job satisfaction were found to be not significant at the $p=.05$ alpha level, $r=.02$. Finally, when queried about school climate and collective effectiveness, YRE staff had a more positive attitude than did those on a traditional calendar in fifteen out of eighteen survey questions, although no significance tests were conducted (Prohm and Baenen, 1996).

\section{Teacher Job Satisfaction}

Some researchers believe satisfaction is a stable trait condition, yet others believe it to be a more transitory state existing for a short period of time. Satisfaction is defined as 'the pleasurable emotional state resulting from the appraisal of one's job as achieving or facilitating one's job values' (Locke, 1981). Overall, satisfaction is a person's affective reaction to his work role. Across the research literature on education, teachers reported dissatisfaction with lack of administrative support, school policy, time, money and student discipline (Kenyeri, 2002).

Hall \& Hord (1987) reports teachers, like members of any organization, are concerned about the environment in which they work. Teachers express concern about class control, their own content adequacy, the situation in which they teach and about evaluations by their supervisors, by their pupils, and of their pupils by themselves. These concerns appear to focus around four major areas: control, rigidity, individualism, and 
professionalism (Smith \& Rowley, 2005; $N=52,000$; Schools and Staffing Survey, NCES, 1999-2000; $\alpha=.77)$. Teacher concern is defined as the "composite representation of the feelings, preoccupation, thought, and consideration given to a particular issue or task" (Hall \& Hall 1987, p. 58). Teachers who are mature in their careers mention different kinds of problems and satisfactions than teachers who have less experience. Significant among all teachers is concern resulting from reform. School reforms can fall under several categories: administrative, curricular, instructional, class scheduling and, in the case of this study, calendar reform. The more personally involved a teacher is with reform, the more likely the teacher response will be intense, cause increased mental activity, thought, worry, analysis, and anticipation (Hall \& Hord, 1987).

Recent research in teacher job satisfaction has utilized several scales. Some of the better known scales include: Teacher Job Satisfaction Questionnaire (TJSQ; Lester, 1987; $N=620$ ), Job Diagnostic Survey (Hackman \& Oldham, 1974; $N=658$; Job Description Index (Balzer et al., 1997), Job Satisfaction Scale (Brayfield \& Rothe, 1951; $N=231$ ), and School and Staffing Survey (NCES, 2000; $N=52,000 ;$ See APPENDIX H for scale psychometrics).

Autonomy, experienced meaningfulness, knowledge of results, and experienced responsibility are predictors of job satisfaction (Brenner, 2004). Brenner administered a modified version of Job Diagnostic Survey (JDS; Hackman \& Oldham, 1974). Hackman \& Oldham (1974) obtained data from 658 employees working on 62 different jobs in 7 organizations. The jobs were highly heterogeneous, including blue-collar, white-collar, and professional work. Both industrial and service organizations were included in the sample, but all were businesses. The organizations were located in the east, southeast, 
and midwest, in both urban and rural settings. Fifty-nine percent of the respondents were male; their median age was twenty-nine, and their education ranged from grade school only to a graduate degree. The other variables measured by the JDS which are predicted to be affected by the job characteristics, including the three critical psychological states, general satisfaction, growth satisfaction, and internal work motivation. For behavioral measures of absenteeism and supervisory ratings of work performance effectiveness, the motivating potential score has a median correlation of -.25 with absenteeism, and of .24 with a summary measure of performance effectiveness. Both relationships are statistically reliable at $p<.05$. The researcher expressed the importance of assessing job satisfaction because literary research maintains satisfaction impacts productivity, work behaviors, turnover, and life satisfaction. Job satisfaction was the dependent variable while teacher characteristics, school characteristics, job dimensions, and psychological states comprised the four classes of independent variables. A correlation design, with hierarchical multiple regression as the primary statistical procedure using the Hulin, Roznowski \& Hachiya (1985) heuristic model provided a comprehensive framework. Components factor analysis was used to construct validate the instrument and reduce the survey items down to a smaller number of predictor variables examined in the multiple regression analysis. Test-retest and coefficient alpha computations assessed the stability and internal consistency of the survey items across seventeen school locations throughout the state to collect data in person. Sampled sites included all school levels and diverse communities. Results indicated the most significant predictors of satisfaction were autonomy and three psychological states: experienced meaningfulness, knowledge of 
results, and experienced responsibility. The researchers suggested implications for practice and future research (Brenner, 2004).

Perceived security, affiliation, self esteem, autonomy, and self actualization as reported by urban high school teachers have high correlation with teachers' degree of job satisfaction (Kreis, 1983; $N=900$ ). This study conducted to explore the relationship between perceived security, affiliation, self esteem, autonomy, and self actualization, and degree of job satisfaction as reported by urban high school teachers. The control variables used include teachers' age, gender, marital status, length of service, salary, family income, school size, absences due to personal and family illness, and perception of available resources. Questionnaires were sent to 900 high school teachers in a major city school district in the northeastern United States. It was found that: (1) the basic relationship between job satisfaction and needs fulfillment was confirmed; (2) a large number (38.4 percent) of the respondents did not fit into the hierarchical arrangement of needs; (3) availability of resources and length of service contributed independently to and served as mild predictors of job satisfaction; and (4) marital status and perception of availability of resources had significant relationships with needs fulfillment. It is concluded that needs are not necessarily fulfilled hierarchically, and that job satisfaction depends upon the fulfillment of individually perceived needs (Kreis, 1983).

Predictors of job satisfaction include several variables (McCaskill \& Others, 1979; $N=682 ;$ Job Descriptive Index (JDI; See APPENDIX H for scale psychometrics). This study investigated teachers' perceived job satisfaction in relation to their work and the supervision they received from the principal. Surveys were collected from 682 teachers from 41 central Texas school districts. The instrument used to evaluate teacher 
perception of job satisfaction and supervision was the Job Descriptive Index (JDI). The canonical correlation was chosen as the most informative and expedient statistical test to select the variables in the JDI that would be the best predictors of teacher satisfaction. Results indicated that job satisfaction was related to having an annual contract rather than a probationary or continuing contract. Teachers working in grades $\mathrm{K}-6$ in the fields of special education, vocational education, and elementary education were more satisfied educators. As class size and total student load increased, teacher work satisfaction decreased. Low salaries caused job satisfaction to decline. Teachers surveyed felt that the principal was a knowledgeable, helpful, and well informed supervisor, but that the principal did not offer enough individual assistance and was not available often enough (McCaskill \& Others, 1979).

Approximately one of every four students who complete a teacher training program never enters teaching or leaves a teaching position within the first five years (Kim \& Loadman, 1994; $N=2,054$; See APPENDIX H for scale psychometrics). Investigating predictors of teacher job satisfaction, the study used survey data from the base year (1988) and follow-ups from 1988 to 1992 of the National Database for Preservice Teacher Education. The 2,054 volunteer respondents were baccalaureate teacher education graduates from ten universities in five states employed in teaching positions. Using multiple regressions to identify statistically significant predictors of job satisfaction, the analysis produced a model with seven statistically significant variables: salary, opportunities for advancement, professional challenge, professional autonomy, working conditions, interaction with colleagues, and interaction with students. The overall feeling of satisfaction with teaching among the respondents was a mean of 4.82 
on the seven-point scale. Generally, only coefficients with a probability of $(p=.05)$ or smaller were considered to be statistically significant. Multiple regression analysis results indicated that the model explained $74 \%$ of the variance in job satisfaction $\left(r^{2}=.72\right)$. All seven independent variables were statistically significant (Kim \& Loadman, 1994). These predictors of teacher job satisfaction are associated with both intrinsic and extrinsic rewards. Extrinsic sources of satisfaction are generally perceived as the factors salary and opportunities for advancement, often controlled by or granted by others. Intrinsic satisfiers are generally classified as professional challenge, professional autonomy, working conditions, interaction with colleagues, and interaction with students. Intrinsic satisfiers refer to factors that make certain activities rewarding in themselves (Kim \& Loadman, 1994).

Curtis (2005) examined the relationship that job satisfaction, collaborative relationships with mentors, general self-efficacy, teacher self-efficacy and organizational efficacy have on new teacher retention. For a quantitative and qualitative study, the researcher used four survey instruments to gather data from respondents: General Perceived Self-Efficacy Scale (Jerusalem and Schwarzer, 2001; Cronbach's alphas ranged from .76 to .90 ), Teachers' Sense of Efficacy Scale-Short Form (Tschanen-Moran and Woolfolk-Hoy, 2001b); School Culture Survey (Gruenert \&Valentines, 1998), and the General Information Questionnaire (Smith \& Ingersoll, 2004). Respectively, the instruments were to: measure an individual's beliefs in his/her ability to organize and execute a course of action with an intended goal in mind, measure teacher self-efficacy in the three areas of student engagement, instructional strategies and classroom management, measure organizational efficacy by examining the shared values/beliefs, the 
patterns of behavior, and relationships in the school, and measure the teachers' intentions to remain in the teaching profession along with independent item measures such as relationships with mentors and job satisfaction. The fourth instrument, General Information Questionnaire, also collected demographic data such as: certification, education level, teaching assignment, and personal information to be use to be used to generally describe the respondents. Using a multiple linear regression tests and collection of minimal qualitative data collected, the researcher concluded that job satisfaction is the most significant predictor of new teacher retention after the first year of teaching (Colgan, 2004). Further, after five years of teaching, teacher self-efficacy, organizational efficacy, collaborative relationships and general self-efficacy were most significant predictors of teachers remaining in the profession. However, general self-efficacy and collaboration with a mentor were not significant predictors of retention of teachers after five years (Curtis, 2005; The Southeast Center of Teaching Quality, 2004).

Interactions among teacher background characteristics and workplace conditions predict significant job satisfaction (Mertler, 2001; $N=969$; Teacher Motivation and Job Satisfaction Survey). The study attempted to explain the current state of affairs with respect to teacher motivation and job satisfaction. The data for this study were collected through the administration of a web-based survey of teacher motivation to 969 elementary, middle and high school teachers during the fall of 2000 . When asked, "What is your overall level of satisfaction with your job as a teacher?" slightly more than $77 \%$ of the teachers responding indicated satisfaction with their jobs. There was no significant difference in the reported levels of satisfaction between females and males, $x^{2}(1, N=$ $951)=3.65, p=.06$ and no significant differences in the distribution of responses for job 
satisfaction based on ethnicity of respondent, $x^{2}(4, N=940)=4.15, p=.39$. There was a statistically significant difference in the distribution of responses for job satisfaction based on age of the respondent, $x^{2}(7, N=957)=24.63, p=.01$. There was statistically significant difference in the distribution for job satisfaction based on the number of years of teaching experience, $x^{2}(4, N=963)=20.31, p=.01$. There was no statistically significant difference in reported job satisfaction between teachers in different school settings and between teachers at different school levels with $x^{2}(2, N=955)=4.24, p=$ .12 and $x^{2}(2, N=955)=5.03, p=.08$ respectively. Mertler expands on a study by the National Education Association that revealed the twenty-five percent (25\%) of teacher respondents expressed dissatisfaction with their current jobs. Given the opportunity to choose a carcer again, thirty-four percent (34\%) of teachers in the study would not choose to enter the teaching profession (Mertler, 1992).

When considering teacher demographics, Ma's $(1999 ; N=2,202)$ research revealed that female teachers were more satisfied with their professional role as a teacher than their male counterparts. Results showed the correlation of job satisfaction and three composite variables: teaching competence $(r=.30)$, administrative control $(r=.37)$ and organizational culture $(r=.29)$. Teachers who stayed in the profession longer were less satisfied with their professional role. Workplace conditions positively affected teacher satisfaction; administration control was the most important, followed by teaching competence and organizational culture. School culture also correlates with teachers' attitudes toward their work. The study that profiled effective and ineffective organizational cultures found stronger school cultures had better motivated teachers. In an environment with strong organizational ideology, shared participation, charismatic 
leadership, and intimacy, teachers experienced higher job satisfaction and increased productivity. Significant interactions between teacher background characteristics and workplace conditions occurred. The gender gap in professional satisfaction grew with increased teaching competence (Ma, 1999).

Correlations exist between teacher quality mentoring, professional development and support, other quantifiable characteristics, scheduled interaction with other teachers in the school and larger community, and formal assessments for new teachers during at least their first two years of teaching and teacher turnover (Alliance for Excellent Education, 2005; MetLife Survey of the American Teacher and National Center for Education Statistics' Public School Teacher Survey). Further, Alliance for Excellent Education (2005) reports that comprehensive induction programs are designed to address the roots of teacher dissalisfaction by providing new teachers with the supports and tools they need to succed (Alliance for Excellent Education, 2005). The findings showed that the time it takes for new teachers to perform at the same level as an experienced teacher on the average of three to seven years - can be shortened when new teachers participate in a comprehensive induction program (Provasnik \& Dorfman, 2005).

\section{Teacher Job Satisfaction in the Context of the YRE Calendar}

Current research reveals generally positive attitudes about the YRE calendar by teachers experienced in YRE calendar reform (Ballinger, 1999; Boyles, 1993; Donahue, 1997; McFadden, 2004; Sanders, 2001). Donahue (1997) surveyed a cluster sample of 163 teachers in a large district of 3000 teachers. The teachers completed a Likert-scaled questionnaire. Results showed an $F$ Value of 19.65 was statistically significant $(p<.01)$ with teachers perceiving YRE program to be a better academic program for students. The 
$F$ Value of 43.47 was statistically significant $(p=.01)$ with teachers stating the YRE program is a better program for at-risk students. However, an $F$ Value of .02 was not statistically significant $(p=.01)$ with no evidence to support the hypothesis that there would be a difference when comparing teacher with more than ten years of experience and teachers with less then ten years experience (Donahue, 1997). Boyles (1993) surveyed teachers in the Mooresville Graded School District. The teacher population consisted of thirty-five teachers, seventeen who worked in the Year-Round Education Program and eighteen teachers who worked in the traditional school calendar classrooms within the school. The questionnaire consisted of four retrospective and prospective questions with fixed responses and a fifth open-ended question.

When asked about satisfaction with teaching - YRE teachers reported 50\% high satisfaction and $50 \%$ medium satisfaction in the past; $86 \%$ high satisfaction and $7 \%$ medium satisfaction with the present; and $86 \%$ high satisfaction and $14 \%$ medium satisfaction in the expected future satisfaction with teaching. Traditional calendar teachers reported $71 \%$ high satisfaction and $29 \%$ medium satisfaction in the past; $38 \%$ high satisfaction and $50 \%$ medium satisfaction with the present; and $71 \%$ high satisfaction and $17 \%$ medium satisfaction in the expected future satisfaction. YRE teachers expressed greater optimism about present and future satisfaction with teaching. Overall, YRE teachers' responses indicated an increased optimism regarding satisfaction with teaching.

When asked about satisfaction with student achievement - YRE teachers repotted $21 \%$ high satisfaction and $79 \%$ medium satisfaction in the past; $64 \%$ high satisfaction and $29 \%$ medium satisfaction with the present; and $93 \%$ high satisfaction and $7 \%$ 
medium satisfaction in the expected future satisfaction. Traditional calendar teachers reported $29 \%$ high satisfaction and $71 \%$ medium satisfaction in the past; $21 \%$ high satisfaction and $71 \%$ medium satisfaction with the present; and 50\% high satisfaction and $46 \%$ medium satisfaction in the expected future satisfaction. YRE teachers expressed greater optimism about present and future satisfaction.

When asked about satisfaction with instructional flexibility - YRE teachers reported $43 \%$ high satisfaction and $50 \%$ medium satisfaction in the past; $72 \%$ high satisfaction and $21 \%$ medium satisfaction with the present; and 79\% high satisfaction and $21 \%$ medium satisfaction in the expected future satisfaction. Traditional calendar teachers reported - $29 \%$ high satisfaction and $67 \%$ medium satisfaction in the past; $25 \%$ high satisfaction and $63 \%$ medium satisfaction with the present; and $33 \%$ high satisfaction and 50\% medium satisfaction in the expected future satisfaction. Overall YRE teachers responses indicated greater past, present, and future optimism with instructional flexibility.

When asked about satisfaction with their teaching effectiveness - YRE teachers reported - 50\% high satisfaction and 50\% medium satisfaction in the past; $72 \%$ high satisfaction and $21 \%$ medium satisfaction with the present; and $86 \%$ high satisfaction and $14 \%$ medium satisfaction in the expected future satisfaction. Traditional calendar teachers reported - $42 \%$ high satisfaction and 58\% medium satisfaction in the past; $46 \%$ high satisfaction and 54\% medium satisfaction with the present; and $75 \%$ high satisfaction and $21 \%$ medium satisfaction in the expected future satisfaction. The expression of optimism by the YRE teachers continues to be consistently conveyed in the final question regarding satisfaction with teaching effectiveness (Boyles, 1993). 
YRE teachers cite advantages as more salary potential, frequent breaks, varied educational opportunities, and a flexible work year. Further, Dejarnett (1994) reviewed nineteen studies focused on teacher satisfaction concerning YRE calendar reform. Fourteen of nineteen studies rated teacher satisfaction as positive. Four studies indicated a neutral teacher response while only one study generated negative teacher response. Approximately seventy-one percent ( $71 \%$ ) felt satisfaction about YRE calendars. Overall, the YRE approach was considered by the school and community to be very positive (Greenfield, 1994).

The working conditions in a YRE calendar school have become more favorable (Elder, 1989). Teachers in YRE schools report greater job satisfaction than teachers in traditional school calendars. This finding is consistent with other studies that show, through surveys and site visits, that teachers demonstrate enthusiasm for YRE calendar reform (Quinlan et al., 1987; White, 1988). Russell (1992) reports that a staff survey shows eighty-two percent $(82.6 \%)$ of the teachers, one hundred percent $(100 \%)$ of the administrators, one hundred percent (100\%) of other certified personnel, and ninety percent $(90 \%)$ of the clerical staff had positive satisfaction about the YRE. Of all staff, seventy-seven percent $(77 \%)$ preferred to work in a YRE school. Sixty-two percent (62\%) of the staff expressed that student attendance was better (though in fact it did not change significantly) and sixty percent (60\%) expressed that student behavior was better (it did improve measurably). Fifty-two percent (52\%) of the staff felt community attitudes are more positive and fifty-seven (57\%) feel that teacher attitudes have improved. 
When asked satisfaction levels about their experiences in YRE, teachers report the following: 1) Allows more time to plan lessons/units; 2) More parent participation; 3) Less stress for teachers and students; 4) School interest is higher; 5) Even breaks mean less stress on students and teachers; 6) Kids are more active, less boredom at home and at school - energy level is higher; 7) Possible availability of different focus on learning; 8) Less stress in term two - envision April break as a good time for a break; 9) Permitted teachers to complete university degrees more easily; 10) Children more ready to return to school after break; and 11) Teachers are more rested after break (Kemp, 1995).

YRE teachers express overwhelmingly positive satisfaction about the YRE (McCasland, 1992). Ninety-eight percent (98\%) agreed or strongly agreed the YRE calendar should be recommended district-wide. Ninety percent $(90 \%)$ felt they made more educational progress with their students on the YRE calendar. Ninety-five percent (95\%) felt a more positive attitude about their work in the new calendar. Ninety-four percent (94\%) felt student and staff morale was higher as a result of the new YRE calendar. Ninety-four percent (94\%) felt less review of previously covered material was necessary under the new YRE calendar. Eighty-six percent ( $86 \%$ ) felt students profited from the enrichment and reinforcement courses taught during the inter-sessions (McCasland, 1992).

Teacher absenteeism is often considered an outgrowth of teacher burnout and satisfaction (Ballinger, 1993; Brekke, 1993; Glines, 1994; Mutchler, 1993; Venable, 1997; Worthen \& Zsiray, 1994). Several YRE studies do indeed show a decrease in teacher absenteeism, although these differences tend not to be statistically significant. Several studies (Barron, 1993; Fardig, 1992; Gandara \& Fish, 1994; Goldman, 1990; 
Kocek, 1996; Loyd; Serifs, 1990; White, 1993) report positive data regarding teacher absenteeism comparisons in YRE calendar and traditional schools. When conducting tests of statistical significance, two studies (Barron, 1993; Kocek, 1996) showed no statistical differences. However, no studies found a greater percentage of teacher absenteeism on YRE schedules than on traditional calendars (Worthen \& Zsiray, 1994; Grotjohn \& Banks, 1993). In fact, it was reported that teachers were absent considerably less on YRE schedules (Brekke, 1984) and research by Quinlan el al. (1987) substantiated the findings that YRE decreased teacher absences considerably (Worthen \& Zsiray, 1994).

Research shows that giving teachers shorter, more frequent breaks benefits them, as well as students, mentally and physically (Natale, 1992). When teachers have less stress and less tension, the results of absenteeism rates drop and enthusiasm rises. The three-week break in YRE allows teachers to regroup, replan, and refresh themselves. They can come into the classroom and start with a new burst of enthusiasm, energy, and exciting lessons (Kocek, 1996). The breaks seem to come right when teachers need them at a YRE school. At the time when teachers' and children's minds have been exhausted to the point of shut down, intersessions are taken and allow for all involved to relax and rejuvenate themselves. Frequent breaks allow teachers more flexibility in planning and more chances to visit other programs, such as conferences and workshops that are customarily held during the school day.

Teacher attendance and absenteeism have a correlation with job satisfaction in a YRE calendar. Based on earlier reviewed research, if teachers become satisfied with the change/innovation to YRE, the less likely they are to miss work, leave or transfer (Kocek, 
1996). The study included teachers from forty-five elementary schools (Chicago Public

Schools, Illinois) located in a predominately low socioeconomic neighborhood comprised of ninety-eight percent (98\%) Hispanic students. These teachers had been employed in the schools when the schools were on traditional calendars and remained in the schools after conversion to the YRE calendar. As a data collection instrument, the researcher used payroll records to determine the number of days teachers were absent from school. After Chi-Square Test on the data, the findings indicated that there was no significant change in teacher attendance after the YRE calendar was implemented in the schools. However, teachers' absenteeism in the YRE calendar schools did not increase (Kocek, 1996).

\section{Teacher Resiliency}

A new and interesting field of study is teacher resiliency. Patterson et al. (2004) defines resilience as "using encrgy productively to achieve school goals in the face of adverse conditions." Patterson et al. used a descriptive research project to build on the conceptual framework that described resilience in school leaders, recently reported by Patterson and colleagues, to examine strategies used by classroom teachers and teacher leaders in building resilience in large urban environments. Further, teacher and teacher leaders identified as successful and resilient by their peers or supervisors were asked what holds them in urban schools. The researchers used a three cycle interview process included pre-interview, interview and review by the respondent for accuracy. Standard qualitative methods were used in the analysis. Results revealed four key findings reported in this paper. Resilient teachers act from a set of values that guides their professional decision-making. They also place a high premium on professional development and find ways, often outside the school district, to get what they need. They provide mentoring to 
others and stay focused on students and their learning. A teacher candidate who gives evidence of resilience, of taking charge to solve problems and find opportunities may add to the school in important ways that bolster student achievement and school success. Selfreports reveal that these teachers believe that the strategies they used to maintain their resilience contributed to their continuing work in urban schools. 1). Resilient teachers have a set of personal values that guides their decision-making; 2). Resilient teachers place a high premium on professional development and find ways to get it; 3). Resilient teachers provide mentoring to others; 4). Resilient teachers are not victims - they take charge and solve problems; 5). Resilient teachers stay focused on the children and their learning; 6). Resilient teachers do whatever it takes to help children be successful; 7) Resilient teachers have friends and colleagues who support their work emotionally and intellectually; 8). Resilient teachers are not wedded to one best way of teaching and are interested in exploring new ideas; and 9). Resilient teachers know when to get involved and when to let go (Patterson et al., 2004).

Tuettemann (1991; $N=574)$ reports that the stressfulness of teaching as an occupation is widely recognized and several studies have been initiated to address its causes. Teachers completed the Teacher Stress Survey, a 360-item questionnaire addressing the following areas: biographical and demographic factors such as age, sex, qualifications and school type; contextual factors such as school classification and subject areas taught; environmental factors such as time and role pressures, student factors, staffstaff and staff-administration relations and professional conditions; teachers' perceptions of their own competence and compensation; and two open-ended questions, the first regarding aspects of work found most stressful and the second inviting teachers' own 
suggestions for alleviating work-related stress. In addition, teachers completed the 30item version of the General Health Questionnaire (GHQ), a measure of stress. When exploring factors rated by secondary teachers as important to their job satisfaction, results indicated that the vast majority of secondary teachers considered classroom success, acknowledgment and appreciation to be important factors associated with job satisfaction. With respect to the tangible rewards of teaching, an interesting male-female difference was apparent. Salary was important to most teachers of both sexes, but statistically more so for the males (Chi-square $(d f 1)=5.2, p<.03$ ) The difference was even more noticeable when promotion was considered. Over half of the male teachers considered this important to their satisfaction but only one-third of the female teachers reported this to be the case. (Chi-square $(d f \mathrm{I})=6.3, p=.03$ ). When exploring the incidence of psychological distress among these teachers, results indicated that nearly half of the teachers are at least moderately stressed. Among these teachers, half again have scored in the "high stress" range, with an overall 23 per cent of males having a GHQ score of ten or more, and a corresponding 20 per cent of females also scoring in this "high stress" category. At the other end of the scale, 59 per cent of female teachers have scored in the "low stress" category - comparing with only 53 per cent of males. However, these differences are not statistically significant (Chi-square $=2.08 ; .30, p<.50$; Tuettemann, 1991).

Kyriacou \& Chien $(2004 ; N=204)$ explored teacher stress amongst teachers in primary schools in Taiwan. The researchers sought to explore the general level of teacher stress, the sources of teacher stress, the coping actions used by teachers, and what actions the teachers think could be taken by schools and the government to reduce teacher stress. 
Researchers designed a questionnaire to explore the general level of teacher stress, the sources of teacher stress, the coping actions used by teachers, and what actions teachers think that could be taken by schools and the government to reduce teacher stress. A total of 203 questionnaires were received. The sample comprised $72 \%$ females and $28 \%$ males. Six percent had less than one year's teaching experience; $30 \%$ : $1-5$ years; 20\%: 6-10 years; and 44\%: over 10 years. Seventy-two percent were classroom teachers, $14 \%$ were subject teachers, and $14 \%$ administrative teachers. Fourteen percent taught in schools with less than 20 classes, $40 \%$ in schools with $21-40$ classes, $8 \%$ in schools with 41-60 classes, and 37\% in schools with over 60 classes. Significant differences between groups were identified using a t-test to compare two groups, and a one-way analysis of variance to compare three or more groups, for $p<.05$. Results indicated about $26 \%$ of the sample reported that being a teacher was very or extremely stressful. This proportion is in line with previous research, which typically report figures between $20 \%$ and $30 \%$ using this scale. No significant differences were obtained on this scale in terms of gender, length of teaching experience, or position held in the school, but those teachers based in large schools (i.e. with over 60 classes) did report a higher level of stress than other teachers. Large schools are often located in cities, tend to be over-crowded and have a lower level of resources per teacher, which may explain this difference (Kyriacou \& Chien, 2004).

\section{Summary}

The purpose of the literature review on teacher job satisfaction is to: (a) briefly review the theoretical foundation of teacher job satisfaction and critically evaluate historical attempts to measure teacher job satisfaction, (b) discuss important substantive 
implications stemming from teacher job satisfaction research that may advance the field, (c) present recent measurement advances, (d) highlight several methodologies that have been utilized in development of teacher job satisfaction instruments and (e) explore teacher job satisfaction in the context of the YRE calendars. Teacher job satisfaction includes consideration of many different student, teacher, administrator, and community variables. Some of the job satisfaction variables reviewed are as follows: control, productivity, work behavior, security, affiliation, self-efficacy, collective efficacy, support, preservice, inservice, motivation, leadership, collaboration, student achievement, and teacher turnover. Brenner (2004) reported on the relationship behavioral measures of absenteeism and supervisory ratings of work performance effectiveness. The motivating potential score had a median correlation of -.25 with absenteeism, and of .24 with a summary measure of performance effectiveness. Duke \& Gansneder (1990) reported that teachers who were involved more frequently in either managerial $(r=.50)$ or technical decisions $(r=.72)$ are more likely to think that they should be involved. Conversely, those who are less frequently involved in either type of decision want to be less frequently involved. While correlations are lower, teachers who are more involved in one type of decision making tend to be involved in the other type $(r=.19)$. Kim \& Loadman (1994) produced a model with seven statistically significant variables: salary, opportunities for advancement, professional challenge, professional autonomy, working conditions, interaction with colleagues, and interaction with students with a probability of $(p=.05)$ or smaller were considered to be statistically significant. Multiple regression analysis results indicated that the model explained $74 \%$ of the variance in job satisfaction $\left(r^{2}=.74\right)$. Hongyun, Lei \& Oingmao (2005) reported that teachers' self-efficacy 
significantly predicted teachers' job satisfaction, work devotion, internal motivation and the satisfaction of colleague relationship and demission tendency. The higher the school's collective efficacy, the school means of teachers' job satisfaction, work devotion, internal motivation and the satisfaction of colleague relationship were higher, and demission tendency was lower. School-level collective efficacy moderated the relationships between self-efficacy and other teachers' characteristics, teachers in higher collective efficacy schools, the teachers' self efficacy was more positively related to teachers' job satisfaction, work devotion, internal motivation and the satisfaction of colleagues than did those in lower collective efficacy schools, but more negatively related to demission tendency. Mertler (2001) found a statistically significant difference in the distribution of responses for job satisfaction based on age of the respondent, $x^{2}(7, N=957)=24.63, p=$ .01 . There was statistically significant difference in the distribution for job satisfaction based on the number of years of teaching experience, $x^{2}(4, N=963)=20.31, p=.01$. Finally, teacher attendance and absenteeism have a correlation with job satisfaction in a YRE calendar. Based on earlier reviewed research, if teachers become satisfied with the change/innovation to YRE, the less likely they are to miss work, leave or transfer (Kocek, 1996). This literature review on teacher job satisfaction research explored the relationship of teacher job satisfaction with school climate, school culture, collective efficacy, and teacher job satisfaction in the context of YRE calendars.

\section{Teacher Intent to Turnover}

Intent to turnover is define as an intent to leave one's present position in an organization within a given time period (Dore, $2005 ; N=325$ ). The study reports that software developer turnover can have disastrous effects on an organization due to the loss 
of business process knowledge, as well as acquired technical skills. Annual rates of turnover in information technology (IT) departments have been estimated at $20 \%$ or more with the cost of replacing technology workers ranging from 1.5 to 2.5 times annual salaries. This study purposely focused only on software developers as opposed to IT employees in general due to the critical nature of their work. The factors leading to turnover intention in this field are poorly understood; therefore, this study was designed to further understand the relationships between job characteristics, job satisfaction, and turnover intention among software developers. 326 web surveys were completed that contained questions relating to job characteristics, job satisfaction, turnover intention, and demographic information. The first four job characteristics are specific to software developers while the last five job characteristics and the job satisfaction scales are from the Hackman and Oldham Job Diagnostic Survey (JDS). Two research questions, sixteen hypotheses, and a theoretical path model were developed to understand which job characteristic variables contribute to the various dimensions of job satisfaction and which job satisfaction dimensions contribute to turnover intention. Additionally, the indirect effects of job characteristics through job satisfaction on turnover intention were also determined. The statistical testing consisted of descriptive and inferential statistical analysis. Bivariate correlations are presented, as well as path analysis, an extension of multiple regression analysis. The results of the study uncovered several factors that can influence turnover intention among software developers. Identified in the study as statistically significant job characteristics that can be influenced by management are training, autonomy, feedback, number of developers, task significance, and skill variety. With the results of this study, management can better understand the unique needs of 
software developers and design development jobs to ensure that these needs are met (Dore, 2005).

When expectations are met, people tend to report higher levels of intent to stay, and organizations are likely to experience lower levels of turnover (Dee, 2002; $N=226$ ). The study utilized a four-item turnover intent measure developed by Price and Mueller (1986). Cronbach's alpha coefficients for the Price and Mueller turnover intent measure have ranged from .85 to .90 (Dee, 2002). Results indicated turnover intent was low to moderate. Respondents reported high levels of autonomy and communication openness. Support for innovation scores was moderate to high. Correlations coefficients indicated a strong, negative relationship $(r=.69)$ between organizational support for innovation and faculty turnover intent. Faculty who perceived high levels of support for innovation reported lower levels of turnover intent. Moderate, negative relationships were found for collegial communication $(r=.60)$ and work autonomy $(r=.44)$. Faculty who perceived high levels of communication openness tended to report lower levels of turnover intent. Faculty who reported high levels of autonomy also tended to report lower levels of turnover intent. One-way analysis of variance (ANOVA) reveled that faculty with 11 or more years of teaching experience had higher levels of turnover intent than faculty with seven or more years of experience $(F=3.52, p=.03)$. Additional one-way ANOVA's showed that turnover intent did not differ significantly on the basis of gender, age, educational level, academic division (workforce development vs. general education), or years at current institution. A block-wise linear regression analysis was used to examine the effects of variables simultaneously. Structural variables entered the model first, 
followed by demographic control variables. The final model explained $54 \%$ of the variation in turnover intent (Dee, 2002).

Watlington et al. (2004: $N=2,129)$ designed a multi-year study covering four South Florida School Districts that examined variables associated with the demographics of teachers who were hired during the 2000-2001 academic year. The purpose was to assess the relationship between teacher retention and various demographic variables. Variables associated with teacher demographics and the relationship of teacher retention to variables such as age, gender, race, preparation and assignment were analyzed. $T$ tests were performed on the quantitative variables and chi-square was used to analyze the nominal variables. The researchers used an alpha of .05 , adjusted for multiple hypothesis testing via Bonferonni's inequality, which left a per hypothesis "testing" alpha of .05/7 = .007. Results indicated very little attrition took place the first year of employment in the four school districts. The overall retention was $95.6 \%$. However, early trends began to show related to retention. Results showed that out-of-state hires were less likely to be retained than in-state hires, $X^{2}(1)=7.91, p<.007$, Contingency Coefficient $=.06$. Older new hires were less likely to be retained than younger new hires, $t(2110)=2.96, p<.007$, $d=.29$. Teachers placed out-of-field were less likely to be retained than teachers placed in-field, $X^{2}(1)=21.90, p<.007$, Contingency Coefficient $=.10$.

Following the second year of teaching, there was a significant drop in the retention rate $(79.8 \%)$. Out-of-state hires were less likely to be retained than in-state hires, $X^{2}(1)=15.57, p<.007$, Contingency Coefficient $=.09$. Males were less likely to be retained than females. $X^{2}(1)=12.25, p<.007$, Contingency Coefficient $=.08$. Teachers placed out-of-field were less likely to be retained than teachers placed in-field, $X^{2}(1)=$ 
$13.63, p<.007$, Contingency Coefficient $=.08$. Alternative preparation teachers were more likely to be retained than approved program and teachers that had no preparation, $X^{2}(1)=23.93, p<.007$, Contingency Coefficient $=.111$. Finally, the overall retention rate for the third year continued a steady decline $(72.8 \%)$. Males were less likely to be retained than females, $X^{2}(1)=13.03, p<.007$, Contingency Coefficient $=.08$. Alternative preparation teachers were more likely to be retained than approved program and teachers that had no preparation, $X^{2}(1)=9.93 . p<.007$, Contingency Coefficient $=.07$. Out-offield teachers continued to be less likely to be retained, compared to in-field teachers, Alternative preparation teachers were more likely to be retained than approved program and teachers that had no preparation, $X^{2}(1)=7.81, p<.007$, Contingency Coefficient $=$ .06. Out-of-state hires were less likely to be retained than in-state hires, Out-of-state hires were less likely to be retained than local teacher hires, $X^{2}(1)=15.17, p<.007$, Contingency Coefficient $=.09$ (Watlington et al., 2004). Much less attention has been given to those who stay, especially the best. Recent research on intent to turnover has utilized several scales. Some of the better know intent to turnover scales are described in APPENDIX I.

Teacher turnover creates a shortage of high quality teachers in low-achieving schools suggests that these teachers are either quitting the profession or transferring to higher performing schools (Guin, 2004). This study examines the characteristics of elementary schools that experience chronic teacher turnover and the impacts of turnover on a school's working climate and ability to effectively function. A purposive sample of fifteen schools, based on their geographic location, demographic characteristics and seven-year average rate of turnover, was used. The school district in this study was a 
large urban district in which many schools are divided by income and race. The school district serves nearly 47,000 students in 97 schools; 70 are elementary and K-8 schools. The district employs 4,500 certified staff, 3,200 of which are currently in teaching positions. The study explored the relationship between teacher turnover rates and other quantifiable characteristics of elementary schools within the district, correlations between teacher turnover rates and student demographics and achievement were examined. Results indicate several significant relationships. With regard to student demographics, there is a significant and positive correlation between teacher turnover rates and the percentage of minority students within a school. Correlations between student performance and turnover rates were also significant, but negative. Schools with higher rates of turnover had fewer students meeting standard on statewide assessments in both reading (Pearson Correlation: -.31, Sig. (2-tailed): .001, $N=418$ ) and math (Pearson Correlation: -.28, Sig. (2-tailed): .001). While these correlations are important to acknowledge, additional statistical analyses beyond the scope of this study are necessary in order to determine the causal effects, if any, between turnover and student performance. In addition to demographic variables and student performance, the correlation between turnover rates and measures of organizational climate from the Staff Climate Survey were also examined, using three years of data (2001-2003). All of the correlations between teacher turnover rates and the six school climate concepts were negative. More importantly, all correlations, with the exception of "Teacher Interactions" were found to be significant. The correlations were as follows: school climate -.17, teacher climate -.16 , principal leadership -.17, teacher influence -.14 , feeling respected .16 , and teacher Interactions -.09. The correlation was significant at the .01 level; Guin, 
2004). With regard to student demographics, there is a significant and positive correlation between teacher turnover rates and the percentage of minority students within a school. Schools that have higher percentages of minority students experience higher levels of teacher turnover. These findings corroborate findings from previous research, indicating that schools with predominately minority students are disproportionately impacted by teacher turnover (Guin, 2004).

Job satisfaction is the most significant predictor of new teacher retention (Curtis, 2005; Smith \& Ingersoll, 2004; $N=52,000$; Teacher Follow-up Survey, National Center for Education Statistics, 1999-2000). The data source was the National Center for Education Statistics' (NCES) Schools and Staffing Survey (SASS). To date, four independent cycles of SASS have been completed: 1987- 88; 1990-91; 1993-94; 19992000. The 1999-2000 SASS comprised about 52,000 elementary and secondary teachers. The study analysis focused on beginning teachers, which we define as those in their first year of teaching in 1999-2000 - a sample of 3,235. New teachers who started their career as regular, fulltime teachers ( 88 percent of the new teachers in 1999-2000) were less likely to turnover than those who began their first teaching job as regular part-time teachers, itinerant teachers, or long-term substitutes. The relative risk of regular full-time teachers leaving as opposed to staying at the end of their first year was about half that of those whose status was as a part-time, itinerant or substitute teacher (relative risk ratio $(r r r=.56, p=.01)$. Teaching status has a similar impact on the relative risk that a teacher will switch schools at the end of the first year as opposed to staying $(r r r=.53, p=.01)$. This makes sense, given new teachers with part-time or irregular status might be likely to be looking for more stable positions either inside or outside their current school. The 
impact of a new teacher's age on their likelihood of leaving or moving is small and statistically insignificant. Prior studies of turnover have found both younger and older teachers more likely to turnover (Smith \& Ingersoll, 2004).

Low achieving schools often end up the least qualified teachers and experience the teacher gap (Quality Counts, 2003). Provasnik \& Dorfman $(2005 ; N=50,000)$. This study states that the most recent national data on public and private school teachers come from two surveys sponsored by the U.S. Department of Education's National Center for Education Statistics (NCES): the 1999-2000 Schools and Staffing Survey (SASS) and the related 2000-01 Teacher Follow-up Survey (TFS). During the 1999-2000 school year, a total of about 3,450,000 teachers worked in public and private elementary and secondary schools across the country—representing about 2.7 percent of the overall U.S. workforce that year. The majority of teachers ( 90 percent) worked full time, four percent worked part time, three percent were itinerant teachers, and less than 0.5 percent worked as long-term substitutes. Eighty-seven percent (3,000,000 teachers) worked in public schools, and 13 percent $(450,000$ teachers) worked in private schools. The 1999-2000 SASS, administered between September 1999 and June 2000, asked a nationally representative sample of over 50,000 public and private school teachers about their work environment, classroom teaching, teaching qualifications, and other individual characteristics. The 2000-01 TFS, administered between January and May 2001, asked a representative sample of over 5,000 SASS participants a series of follow-up questions about how their job had changed since the previous year. Respondents included those who continued teaching the year after completing the initial SASS and those who left the 
profession. Unless otherwise noted, the data presented in this special analysis come from the 1999-2000 SASS or the 2000-01 TFS (Provasnik \& Dorfman, 2005).

Provasnik \& Dorfman (2005) reported that at the end of the 1999-2000 school year, public and private schools lost a total of about 550,000 teachers (or 16 percent of the teacher workforce) due to teacher turnover. Roughly 270,000 of these teachers $(8 \%)$ transferred to a different school, and the other 280,000 ( 8 percent) left teaching for various reasons. The teachers who left teaching_or "leavers" for the purpose of this analysis - consisted of teachers who retired ( 2 percent), took a job other than elementary or secondary teaching (4 percent), returned to school for further education (.3\%), left for family reasons (e.g., to raise children or take care of other family members) (1\%), and left for miscellaneous other reasons (1\%). At the end of 1999-2000, leavers who retired, naturally, tended to be older teachers, who, on average, had taught for 29 years in elementary, middle, or high school. The average age of retirees was 58, though 25 percent were 50-54 years old when they retired, 38 percent were 55-59 years old, and 36 percent were 60 or older 30 . The apparent difference between the proportion of females among retirees and continuing teachers was not statistically significant. Likewise, there was no measurable difference between the percentages of retirees and continuing teachers who were highly qualified and were teaching out-of-field due to the small sample size and large standard errors. Leavers who took another job other than elementary and secondary teaching were disproportionately male when compared with continuing teachers ( $32 \%$ vs. $25 \%$ ). On average, these leavers were 39 years old and had 10 years of teaching experience before they left. These leavers were less likely to be highly qualified than teachers who continued to teach in the same school (50\% vs. $63 \%)$ and were twice 
as likely to have been teaching out-of-field ( $24 \%$ vs. $11 \%)$. Teachers who transferred, as noted earlier, tended to be younger and less experienced than continuing teachers. In particular, beginning teachers (those with 3 or fewer years of teaching experience) were more likely to transfer than teachers with 10 or more years of experience (data not shown). Transfers were less likely to be highly qualified than teachers who continued to teach in the same school (55\% vs.63\%) and were more likely to have been teaching outof-field before they transferred (15\% vs.11\%; Provasnik \& Dorfman, 2005).

Hellman (1997) reported voluntary turnover of desirable employees is generally considered detrimental to the organization, both in replacement costs and work disruption. The study look at two issues: First, across levels of job satisfaction, would older employees would be less inclined than younger ones to leave federal service. Second, would employees with higher levels of tenure be less inclined than employees with lower levels of tenure to leave federal service across levels of job satisfaction. Intent to leave, though not a perfect predictor of turnover, is an important precursor to turnover across many types of occupation (Steel \& Ovalle, 1984). Results indicated that at least two of the age category correlations were different, $x^{2}(4, N=5,525)=69.50 ; p<.01$. Next, the researcher applied the chi-square test to two sets of correlations. Set 1 included the age categories 29 and younger, 30 through 39, and 40 through 49 . Set 2 included the age categories 50 through 59 and 60 and over. The correlations in Set 1 were not significantly different, $x^{2}(2, N=4,525)=3.16 ; p>.05$. Similarly, for Set 2 , these correlations were not significantly different, $x^{2}(1, N=1,328)=0.15 ; \mathrm{p}>.01$. Thus, with increasing age, federal employees were less likely to leave the organization across levels of job satisfaction (Helman, 1997). 
To test the hypothesis that employees with more tenure would be less likely to leave the organization regardless of their level of job satisfaction, the researcher calculated a breakdown of correlations by years worked in the federal agency. At least two of the correlations were different, $x^{2}(5, N=5,571)=78.43 ; p<.01$. The correlations suggested that the correlations could be initially grouped into two sets: Set $1=$ those employees with less than 10 years with the federal agency; Set $2=$ those employees with 10 or more years with the federal agency. Subsequent chi-square analysis could then be implemented. For Set 1, the correlations did not differ significantly, $x^{2}(2, N=2,915)=$ 1.72; $p>.05$. However, for Set 2, at least two of the correlations were different, $x^{2}(2, N$ $=2,656)=14.96 ; p<.05$. The correlations in also suggested that employees with 10 to 20) years' service with the U.S. federal agency might be grouped as a set and subjected to subsequent analysis. For this set, the correlations were not significantly different, $x^{2}(1, N$ $=1,497)=.72 ; p<.05$. Overall, for the three sets of tenure categories (Set $1=$ less than 10 years; Set $2=10$ through 20 years; and Set $3=$ more than 20 years), U.S. federal employees with higher levels of tenure were less likely than their counterparts in the private sector to consider leaving the organization across levels of job satisfaction (Helman, 1997).

In an effort to promote clarity, theorists have sought to explain factors that predict turnover. Intent to stay in current employment is the variable with the greatest influence on turnover (Gauci \& Norman, 1997). This review of the nursing literature aims to identify the factors with the greatest influence on turnover and absence of qualified nurses, possible common factors influencing both, and the relationship between absence and turnover. A hypothetical model grounded in the literature which depicts the expected 
relationships between these variables is presented for testing in an empirical study. The review identifies intent to stay in current employment as the variable with the greatest influence on turnover. Intent to stay is in turn most strongly associated with job satisfaction. However, job satisfaction is identified as possibly influencing both absence and intent to stay and kinship responsibility is identified as a common antecedent of absence and intent to stay. Similarly, absence is identified as an antecedent to turnover. Thus, it is expected that absence would be positively related to turnover and negatively related to intent to stay. Understanding such relationships should allow identification of management strategies to reduce both turnover and absence. Intent to stay is in turn most strongly associated with job satisfaction. Other variables are identified by studies as having an influence on intent to stay, but are not supported by the results of other studies; exceptions are pay and opportunity for alternative employment. The relationship between job satisfaction and absence is unclear and requires further investigation (Gauci \& Norman, 1997). However, job satisfaction is identified as possibly influencing both intent to stay and absence are identified as a common precursor of absence and intent to stay. Similarly, absence is identified as a precursor to turnover. Thus it is expected that absence would be positively related to turnover and negatively related to intent to stay. Understanding such relationships should allow identification of management strategies to reduce both turnover and absence (Gauci \& Norman, 1997).

Flowers $(2004 ; N=500)$ reported the rate of teacher attrition has reached epidemic proportions in some states and both federal and state governments are researching reasons as to why so many public school teachers are leaving their profession and what can be done to retain them. It is estimated that thirty to fifty percent (30-50\%) 
of public school teachers leave their profession within three to five years for better paying jobs. The purpose of this research is to identify some of the main reasons that teachers are leaving their profession so early in their careers in the state of Texas and what can be done to influence them to remain. A validated survey questionnaire was used to collect the data from a population sample of public school teachers that left the teaching profession at the end of the 2000-2001 school year. The cost of teachers, like the cost of everything else in our society, follows the economics of supply and demand. Texas colleges and universities annually graduate only about half the number of teachers required to replace the 60,000 Texas teachers who leave each year through attrition. The Alliance for Excellence in Education (2005) research identifies what state, county, Education Service Center regions, and school districts in the state of Texas can do to influence teachers to remain in the teaching profession. This study indicates that the most significant factors that caused teachers to leave the teaching profession were (a) inadequate salary, (b) too much paperwork, (c) too much emphasis on preparing students for standardized tests, (d) class size too large, (e) too many hours after school day working on lesson plans and grading papers, (f) lack of guardian and parental support, (g) salary opportunities in other careers, (h) amount of classroom planning and preparation time required for class, (i) too much job stress, (j) frequency of discipline problems in the classroom and school, (k) amount of no-teaching duties required, (l) burnout, and (m) lack of respect from students. This study indicates there should be more focus on retaining teachers rather than recruiting additional teachers (Flowers, 2004).

Neighborhood schools with high poverty rates experienced greater rates of teacher turnover (Chicago Acorn, 2003; $N=2,377$; Teacher Service Record/Certification 2000- 
2001 and Teacher Service Record/ Certification 2001-2002). The study examines the cost of teacher turnover more in the terms of loss of continuity and coherence in a school's curriculum and a disruption of on-going efforts at school reform. School districts are experiencing the added burden of cost and inefficiency that result in threat to the fiscal health of districts that are already financially strapped. Chicago Acorn (2003) found that the greatest burden of turnover fell on low-performing and economically challenged schools where student performance may suffer the greatest. Funds that are drained by turnover-related costs could be used in more productive way to benefit students, teachers, schools, and communities. The cost of turnover to the Chicago Public Schools (CPS), based on the CPS salary schedule, was estimated using three models employed in teacher turnover research: Model I: (20\% of the leaving teacher's salary - $\$ 10,329.40$, Model 2: (150\% of leaving teacher's salary - $\$ 77,470.50$, and Model 3: (2 1/2 times the teacher preparation cost - $\$ 63,689.00)$. Total estimates of turnover cost for CPS 545 leaving teachers for 2001-2002 was $\$ 5,629,523$ based on Model 1, $\$ 42,221,422$ based on Model 2, and $\$ 34,710,505$ based on Model 3 (Chicago Acorn, 2003).

Teachers experience difficult times and adverse situations that could cause them to leave the profession almost daily (Grant, 2006). Teachers are faced with uncomfortable situations each day. Teachers may feel unprepared to deal with stressful situations that arise out of classroom management issues. They may not have the will and commitment to teaching in order to overcome these obstacles. Therefore, teachers may feel that they cannot make a difference in the success of students. Efficacy influences teachers' persistence when things do not go smoothly in the classroom and their resilience in the face of setbacks. Teachers who return year after year feel that they can overcome 
setbacks. They learn from their experiences when a lesson or the handling of a discipline issue does not go smoothly (Tschammen-Moran, Woolfolk \& Hoy, 1998).

Career-related self-efficacy, assertiveness, stress, and gender are among the variables which affect career choice and development (Troutman \& Others, 2000). Increased stress, burnout, and lack of job satisfaction may contribute to a decline in work performance, absenteeism, and intent to leave one's job or field. Researchers have undertaken to determine organizational, job-specific, and personal predictors of level of burnout among employees. Employees, who are highly committed to the organization work harder, are absent less often and are less likely to leave the organization. When an employee leaves, the organization incurs significant costs to recruit and train a successor. The new employee is often less productive initially and co-workers may be negatively affected as well. Thus earning employees' commitment and reducing turnover are important organizational goals. Significant correlation was found between student performance and turnover where schools with higher rates of turnover had fewer students meeting on statewide assessment in both reading and math (Guin, 2004). Smith \& Rowley (2005) explored the extent of the relationship between teacher participation in decision making and their participation in professional development mediated by school environments with more teacher collaboration, administrative support, and a positive social climate. The data for this study come from National Center for Educational Statistics' (NCES) nationally representative SASS, along with its supplement, the Teacher Follow-Up Survey (TFS). Although individual teachers' role in setting school policy was more important than their level of control over classroom practices in predicting how much content-related professional development (PD) was taken, increases 
in both forms of decision making were associated with a reduced likelihood of turnover. For example, a one standard-deviation increase in teachers' perceptions of their level of control over classroom practices is associated with a $8 \%$ reduction in the odds that they turn over $(\beta=-.02, p<.001)$; a one standard-deviation increase in teachers' perceptions of their level of influence over school policies is associated with an $13 \%$ reduction in the odds that they turn over $(\beta=-.02, p<.001) .10$ At the school level, school mean control over classroom practices was associated with an $10 \%$ reduction in the odds of turnover ( $\beta$ $=-.04, p<.001)$, while the coefficients for school mean teacher influence over policy and the ratio of teachers' influence on policy to that of others were small and not statistically significant. Among other school organization variables, administrative support had the strongest association with reduced turnover-a standard deviation increase was associated with an $8 \%$ education in the odds of turnover $(\beta=-.03, p<$ $.001)$, while an atmosphere of collaboration $(\beta=-.04, p<.001)$ and an improved social climate $(\beta=-.008, p<.05)$ were associated with a smaller reduction in the odds of turnover (6\% and $7 \%$, respectively). Although a standard deviation increase in participation in PD (36.5 hours) is associated with a $9 \%$ reduction in the odds of turnover $(\beta=-.003, p<.001)$, the coefficient on school average participation in PD is not significant. As researchers had grand-mean-centered hours of PD at the teacher level, they could interpret the coefficient on school mean participation in PD as a contextual effect (the between-school effect minus the within-school effect. Furthermore, adding PD has little impact on the decision-making and school organization variables reported, suggesting that these variables have a direct effect on turnover and an indirect effect through their impact on participation in PD. The researchers explored the degree to which 
organizational context influences the strength of the relationship between PD participation and the likelihood of turnover (i.e., a cross-level interaction), holding constant all of the variables included in the prior model. Among these school organization variables, school mean teacher influence over policy has a statistically significant interaction with hours of participation in $\mathrm{PD}(\beta=-.0005, p<.05)$, although the effect is sizable. For example, the main effects of a one standard-deviation increase in PD participation and a one standard deviation increase in school mean teacher influence over policy are associated with a reduction in the odds of turnover of $12 \%$-adding in the interaction term increases the reduction in the odds of turnover to $47 \%$. In other words, the impact of taking PD on reducing the likelihood of turnover is considerably stronger in schools where teachers have greater influence over setting school policy. Interactions between participation in PD and school mean teacher control over classroom practices, school mean teacher influence over school policy, relative teacher influence compared to other decision makers, level of administrative support in the school, and the level of collaboration in the school were not statistically significant (Smith \& Rowley, 2005).

Additional findings indicated that several schools exceeded the seven year district average turnover of nineteen percent. Additionally, when considering student demographics, there was a significant and positive correlation between teacher turnover and the percentage of minority students within a school (Provasnik \& Dorfman, 2005). In clearer terms, schools with higher percentages of minority students experience higher levels of teacher turnover thereby disproportionately impacting schools with predominately minority student base. Provasnik \& Dorfman (2005) found that schools 
with fifty percent $(50 \%)$ of more minority students experience turnover rates at twice the rate of schools with lower minority populations.

Smith \& Ingersoll $(2004 ; N=52,000)$ addressed the need for empirical evaluation of the effects of induction on beginning teacher turnover. Researchers examined whether first-year teachers who participated in or received induction activities and supports, such as mentoring, collaborative activities with other teachers, and additional resources, were more or less likely to stay with their teaching jobs the following year. Unlike most previous empirical research, our sample was drawn from a cohort including all beginning teachers, thus allowing us to compare the retention of both those who did and those who did not participate in various induction activities. Moreover, unlike most previous empirical research, we utilize nationally representative data and control for a wide range of teacher and school factors. The data source for the study was the National Center for Education Statistics' (NCES) Schools and Staffing Survey (SASS). To date, four independent cycles of SASS have been completed: 1987- 88; 1990-91; 1993-94; 19992000. The study questions were as follows: How widespread are induction programs across the nation, and has their prevalence increased over the past decade? How many beginning teachers participate in different kinds of induction and mentoring activities? What are the rates of turnover among beginning teachers? What are the effects of different kinds of mentoring and induction activities on the likelihood that beginning teachers depart their jobs? Results indicated that new teachers who started their career as regular, fulltime teachers ( 88 percent of the new teachers in 1999-2000) were less likely to turnover than those who began their first teaching job as regular part-time teachers, itinerant teachers, or long term substitutes. The relative risk of regular full-time teachers 
leaving as opposed to staying at the end of their first year was about half that of those whose status was as a part-time, itinerant or substitute teacher (relative risk ratio $(r r r=$ $.56, p=.01$ ). Teaching status has a similar impact on the relative risk that a teacher will switch schools at the end of the first year as opposed to staying $(r r r=.53, p=.01)$. This makes sense, given new teachers with part-time or irregular status might be likely to be looking for more stable positions either inside or outside their current school. The impact of a new teacher's age on their likelihood of leaving or moving is small and statistically insignificant. Teachers whose main assignment field was special education were far more likely than other teachers to turnover. For instance, the odds that a special education teacher left as opposed to stayed were about $21 / 2$ times higher than for other teachers $(r r r=2.67, p=.001)$. Across models, new teachers whose main assignment field was math or science were about 10 percent more likely than other teachers to leave, although the coefficients are not statistically significant. Conversely, teachers whose main assignment fields were bilingual education or English as a second language were less likely to leave, although again these differences were not statistically significant. Neither gender nor minority status were statistically significant in predicting turnover, although in the SASS sample males were slightly less likely than females to leave and more likely to move; minority teachers were more likely than white teachers to leave and less likely to move. Higher earnings from all school-related jobs were also negatively associated with both moving and leaving, although, again, the impact was not statistically significant. School-level poverty was also associated with an increased risk of beginning teachers leaving at the end of their first year $(r r r=1.01, p=.02)$. For example, a 50 percent increase in the percentage of students approved to receive free or reduced price lunches 
(e.g., the difference between a school where a quarter of the students are poor versus a school where three quarters of the children are poor) increased the risk of a new teacher leaving by about 50 percent. School poverty was not associated with the risk that a beginning teacher switched schools, however. Beginning teachers in middle schools were nearly twice as likely as their counterparts in elementary schools to leave after the first year $(r r r=2.33, p=.001)$, while teachers in high schools were about 50 percent more likely to leave $(r r r=1.57, p=.06)$. Differences in the risk of leaving were not statistically significant for teachers in combined schools compared to elementary school teachers, however. As with the descriptive statistics above, there was little difference in the likelihood of turnover by school location (urban, suburban, small/town rural). School size also did not have a statistically significant impact (Smith \& Ingersoll, 2004).

Three structural variables (level of faculty autonomy, amount of support for faculty innovation, and degree of communication openness in the college) have significant relationships with faculty turnover intent in an urban community college (Dee, 2002; $N=149 ;$ Measure of Turnover Intent. Price \& Mueller, 1986; See APPENDIX I for scale psychometrics). The researcher identified numerous career stressors that are particularly prevalent among urban college faculty (increasing external demands, difficulty establishing a college community) and highlights the potential influence of these factors on faculty turnover. High rates of faculty dissatisfaction and turnover can be costly to the reputation of any institution and to the quality of instruction. "Under conditions of high turnover, faculty morale is likely to suffer, and the quality of studentfaculty interactions - a key factor in college student retention- will be affected" (Dee, 
p.3). The reputation of a school, especially K-12 schools, is very important given the difficulties schools experience trying to meet NCLB requirements.

Many new teachers find that they are unprepared for the reality of the classroom. Croasmun, Hampton \& Hermann (2006) found that beginning teachers leave the teaching field because of the inability to cope with teaching problems. Discipline, difficulties with parents and lack of sufficient or appropriate teaching materials are among the problems experienced by beginning teachers. In addition, beginners are often given the most difficult teaching assignments. Once they leave the university classroom setting, novice teachers often receive little or no support and find that their teacher education programs ill-prepared them for the realities of teaching. University graduates who are satisfied with their teacher preparation programs are more likely to stay in teaching (Croasmun et al., 2006).

Another reason many new teachers leave is that teaching, as a profession, has been slow to develop a systematic way to induct beginners gradually into the complexities of a job that demands hundreds of management decisions every day. Terms like intern and trainee are used in other professions to identify a beginner who has received training in the profession and who earns a stipend by participation in limited experiences under expert supervision. However, in the teaching profession, these terms are often used to identify interns and trainees who have full teaching responsibilities. To retain new teachers, particularly those teaching in inner-city schools, teachers must be introduced to the profession humanely, in ways that engender self-esteem, competence, collegiality, and professional stature (Croasmun et al., 2006). Another possible factor involved in the higher attrition rate for beginning teachers is the initial level of 
commitment to the teaching profession. Some prospective teachers enter the profession with a positive attraction for teaching and plan to make it a long-term career. Others enter the profession with the intent of staying only a few years and plan to quit working altogether, or to use the skills gained from their education to pursue interests in other fields. Further, Weiss (1999) found that teachers highly involved in their work attributed their decision to stay in teaching more the result of supportive workplace conditions than to pay. Supportive workplace conditions included appropriate workload, opportunities for collegial interaction, professional development, participation in decision making, and support for student discipline (Weiss, 1999).

Demographic, structural, and perceptional variables predict the intent of administrative staff to stay or leave their current positions (Johnsrud \& Rosser, 1997; $N=$ 1, 293; See APPENDIX I for scale psychometrics). The study tried to identify what combinations of demographic, structural, and perceptual variables best discriminates mid-level administrators who intend to stay from those administrators who intend to leave their current positions in higher education. Researchers used discriminant analysis to find a minimum number of variables need to predict the intent of mid-level administrators staying or leaving their current positions. The first test of the data was the priori classification to examine the underlying structure. On discriminant analysis function was calculated with chi square $(8 d f)=85.97(p<.001)$. The canonical correlation of .41 indicated the Discriminant function provided a moderate degree of association. The group centroids (means) of -.64 and .31 suggests that the discriminant function separates nicely those individuals who intend to stay from those who intend to leave. The strength of the standardized coefficients suggests the importance of each 
predictor in classifying mid-level administrators after controlling for the effects of other predictors. The coefficients indicate that level of morale (.65), opportunity to advance (.51), and the years employed (.37) contribute most strongly to the classification of group membership. Administrators who intend to leave have a lower mean on morale $(x=8.67)$ than those who intent to stay $(x=10.00)$. People who feel stuck are more likely to intend to leave $(x=1.29)$ rather than stay $(x=1.57)$ in the positions (Johnsrud \& Rosser, 1997).

Data analyses summary indicated: “(1) administrators intending to leave had lower mean morale than those intending to stay; (2) people who felt stuck were more likely to intend to leave than intend to stay; (3) mid-level administrators intending to leave were more likely to work in student affairs divisions than in academic, business, or external affairs; (4) age and recognition of competence contributed moderately to predictions of who would stay or who would leave; (5) among demographic variables, only age and years of employment discriminated stayers from leavers, with older administrators being more likely to stay; (6) gender, race/ethnicity, and minority status did not predict leaving or staying behavior; (7) working at a research institution, as opposed to a community college or baccalaureate institution, was associated with intentions to leave the university; and (8) perceptions regarding the opportunity for advancement, working conditions, recognition for competence, and morale clearly discriminated administrators intending to stay and those intending to leave" (Johsrud \& Rosser, 1997, Abstract).

\section{Teacher Intent to Turnover in the Context of the YRE Calendar}

Kenyeri (2002), Lui \& Li (2005) and VanderHooven (1994) report a relationship between job burnout, absenteeism and intent to turnover for teachers. Results from the 
Maslach Burnout Inventory and Intention to Quit Questionnaire on teachers (Lui et al., 2005, $N=717$; VanderHooven, $N=293 ;$ Kenyeri; $N=200$ ) indicated twenty-five percent $(25 \%)$ teachers had moderate intention to quit and six percent $(6 \%)$ had high intention to quit respectively. The teachers in middle school had higher intent to turnover than those in elementary school; teachers with higher education had higher intention than those with lower education level; the intention of teachers with teaching experience less than fifteen years was higher than those with over twenty-one years; the intention of teachers below thirty-four years old was higher than those over forty-six years. The more serious emotion exhaustion and depersonalization were, the higher intention to quit would be; the less personal accomplishment was, the lower intention to quit would be. Burnout predicted $16.5 \%$ of intention to quit. Burnout has significant effect on intent to turnover. Young, competent teachers tend to turnover (Lui \& Li, 2005). Survey results measuring burnout subscales emotional exhaustion, depersonalization, and personal accomplishment from 141 teachers in the YRE calendar program and 152 teachers in the traditional agrarian program concluded that there was a significant difference in the burnout subscale for emotional exhaustion between the $45 / 15$ calendar teachers and traditional agrarian calendar teachers. Teachers in traditional schools report higher levels of emotional exhaustion (VanderHooven, 2002). Further, Kenyeri (2002) indicated that the subscale of personal accomplishment was significant with teacher burnout and socioeconomical status schools.

\section{Costs of Turnover}

Several basic methods for estimating the cost of turnover define the cost as a percentage of annual salary plus the cost of benefits. Hauenstein (1999) of Advantage 
Hiring postulates the turnover cost per employee is equal to roughly twenty-five percent $(25 \%)$ of the annual salary of the leaver plus the amount the company invests in benefits for the leaver (Hauenstein, 1999). In this model, the cost for benefits averages thirty-five percent (35\%) of the leaver's annual salary. The author notes that this is considered a conservative estimate. In Gately (2005) the cost of employee turnover model asserts that turnover costs equal roughly twenty-five percent ( $25 \%)$ of the leaver's annual salary plus the cost of benefits. Gately suggests that the company's investment in employee benefits is approximately 30 percent (30\%) of the leaver's annual salary. Ettorre (1997) estimates turnover cost as twenty-five percent (25\%) of the leaver's annual salary. The United States Department of Labor estimates that costs to replace an employee average thirtythree percent (33\%) of the new hire's salary (Brannick, 1999). These estimates represent one way in which employee turnover costs may be calculated, but due to the lack of itemization of costs, the estimates serve as a rough "best guess" at the cost of turnover for an organization. The estimates are conservative because they do not calculate the actual costs an organization invests in termination, recruitment and hiring, substitutes, learning curve loss, and training. Once all of these costs are calculated, the actual cost of turnover can reach as much as double the annual salary and benefits of the leaver (Fitz-enz, 1997).

In 2007, Texas (like many other states) is facing a serious teacher shortage as a result of increased enrollment coupled with decreasing retention of teachers. Texas has attempted to calculate costs when teachers leave the profession or change school districts. This cost of teacher turnover represents financial cost to public education beyond the cost mostly associated with operating schools (The Texas State Board for Educator Certification, 2000). These turnover costs result in loss of resources that might otherwise 
have been used to improve the effectiveness of instruction. Additionally, turnover has a personal cost for teachers who have invested significant time and resources to prepare for the teaching profession. This state report reports the following key findings:

- In, the 1998-99 school year, Texas school districts struggled to fill over 63,000 teaching positions, 24 percent of Texas teaching positions;

- The attrition rate for 1998-99 is estimated to be 19 percent for beginning teachers;

- The current teacher shortage presents a significant challenge for Texas schools (p. 1).

Contributors to the cost of teacher turnover include separation costs, hiring costs, training and support costs. Conservative industry models for calculating turnover cost estimate the cost of one employee to be twenty-five percent $(25 \%)$ of the individual's salary. Other models estimate the cost as high as two hundred percent (200\%; Texas State Board for Educator Certification, 2000). Further conclusions in this study include (1) resource allocations change when turnover increases, (2) teacher turnover presents an enormous expense to Texas school districts that cannot be recouped, and (3) costs associated with teacher turnover in Texas school districts can be reduced but not eliminated with practices that encourage teachers to remain in the profession such as strong support systems, stipends for participating in professional development support and advanced certificates (p. III).

Five different turnover cost models were discussed by Texas State Board for Educator Certification (2000). See APPENDIX J for the five models and descriptions of the calculating process. 


\section{Summary}

The purpose of the literature review on teacher intent to turnover is to: (a) briefly review the theoretical foundation of teacher intent to turnover and critically evaluate historical attempts to measure teacher intent to turnover, (b) discuss important substantive implications stemming from teacher intent to turnover research that may advance the field, (c) present recent measurement advances, (d) highlight several methodologies that have been utilized in development of teacher intent to turnover instruments and (e) explore teacher intent to turnover in the context of the YRE calendars. This chapter has explored the workplace conditions that surround and impact teachers' decisions to leave or remain in the profession. It has discussed the social cognitive theory of self-efficacy and the relationship of school climate, school culture, teacher efficacy, collective efficacy, teacher job satisfaction and teacher intent to turnover in the context of the YRE school calendar. Dee (2002) correlations of intent to turnover and school climate indicated correlations coefficients indicated a strong, negative relationship $(r=.69)$ between organizational support for innovation and faculty turnover intent. Faculty who perceived high levels of support for innovation reported lower levels of turnover intent. Moderate, negative relationships were found for collegial communication $(r=.60)$ and work autonomy $(r=.44)$. Additionally, Dee (2002) reveled that faculty with 11 or more years of teaching experience had higher levels of turnover intent than faculty with seven or more years of experience $(F=3.52, p=.03$ ). Daugherty et al. (2005) found correlations that suggest school climate is directly linked to teachers' perceptions of a principal's effectiveness. For example, if teachers perceived that principals "used the most appropriate response for each situation" (high EFF scores) then they characterized the 
school to have good communications (.37), participatory decision-making (.37) and high levels of advocacy for teachers (.41). Rowley (2005) reported that a one standarddeviation increase in teachers' perceptions of their level of control over classroom practices is associated with a $8 \%$ reduction in the odds that they turn over $(\beta=-.02, p<$ $.001)$; a one standard-deviation increase in teachers' perceptions of their level of influence over school policies is associated with an $13 \%$ reduction in the odds that they turn over $(\beta=-.02, p<.001)$. Smith \& Ingersoll (2004) indicated that new teachers who started their career as regular, fulltime teachers (88 percent of the new teachers in 19992000) were less likely to turnover than those who began their first teaching job as regular part-time teachers, itinerant teachers, or long term substitutes. The relative risk of regular full-time teachers leaving as opposed to staying at the end of their first year was about half that of those whose status was as a part-time, itinerant or substitute teacher (relative risk ratio $(r r r=.56, p=.01)$. Teaching status has a similar impact on the relative risk that a teacher will switch schools at the end of the first year as opposed to staying $(r r r=.53, p$ $=.01$ ). Johnsrud \& Rosser (1997) found that the coefficients (demographic, structural, and perceptual variables) indicate that level of morale (.65), opportunity to advance (.51), and the years employed (.37) contribute most strongly to the classification of group membership. Administrators who intend to leave have a lower mean on morale $(x=8.67)$ than those who intent to stay $(x=10.00)$. People who feel stuck are more likely to intend to leave $(x=1.29)$ rather than stay $(x=1.57)$ in the positions (Johnsrud \& Rosser, 1997).

While the current literature review provides valuable insight into the relationship between teacher turnover and other variables, it is only a brief snapshot to gain a better understanding of teacher intent to turnover in the context of YRE, it would be necessary 
to have more data from teachers who have worked in the context of YRE over multiple years. Such data would allow researchers to devote more attention to the effects of teacher turnover, both on a school's ability to function effectively and on student outcomes. It is critical for school district leadership and school boards to recognize high rates of teacher turnover may result in significant financial and instructional costs at the state, community, district, and school level. While turnover is normally associated with questions of a new incoming teacher supply and quality, it is important to acknowledge that teacher turnover may have a negative impact on schools as organizations through the loss of teachers already in their early years of the profession. Based on the review of literature, schools with high rates of turnover face serious organizational challenges, including the failure to establish effective and consistent instructional programs and a lack of commitment among teachers to continue in the profession resulting from perceptions of school climate, school culture, teacher efficacy, collective efficacy and teacher job satisfaction. Unfortunately, high teacher turnover schools, especially urban schools, are most likely to serve the students in most need of help and have the greatest problem in retaining qualified and experienced teachers. District and school level educational policies, including monitoring teacher turnover and providing workplace inducements for teachers, will help identify and aid these turbulent schools in establishing the stable teaching staff necessary for building the personal relationships with students and teachers and organizational competence needed for retaining staff, school improvement and student achievement gains. When policymakers consider time as a workplace condition and retaining teachers, further study of the teacher workplace in the YRE context should be pursued. Teachers perceived the YRE calendar as more 
professional, giving them a better public image (Shields \& Oberg, 1999), found that flexible, creative school administrators, part-time employment possibilities, and intersessions or breaks spaced throughout the year have added up to a positive workplace for teacher retention and job satisfaction. The YRE calendar model has the potential to help schools attract and retain top-notch teachers. 


\section{CHAPTER III}

\section{METHOD}

This chapter describes the research design used in this study including the participants, survey instruments, research procedures, data collection procedures, and data analyses employed to investigate data on the relationships between school climate, school culture, teacher efficacy, collective efficacy, job satisfaction and intent to turnover in select Kentucky school districts operating on YRE calendar reform. The purpose of this chapter is to provide the reader with enough detail to replicate this study in other school calendar environments. The research questions that guided this study are as follows:

1. To what extent do school climate, school culture, teacher efficacy, collective efficacy, teacher job satisfaction relate to teacher intent to turnover in the YRE context?

2. To what extent does teachers' length of employment in a YRE calendar relate to teacher intent to turnover?

3. After controlling for the background variables (i.e., gender, age, years of YRE teaching experience), what is the unique contribution of school climate, school culture, teacher efficacy, collective efficacy, teacher job satisfaction and intent to turnover? 


\section{Participants}

The target population included teachers working in year-round education calendar schools. A national sample would have been ideal for the study; however given time and cost constraints, the participants of this study will consist of a convenience sample of approximately 1000 teachers (Kentucky School Directory, 2007) employed in the following select YRE calendar school districts in Kentucky: School District A, School

District B, School District C, School District D, School District E, School District F, School District G, School District H, and School District I. Convenience sampling of participants was used and participation was voluntary. This nonprobability method is often used in social science research not because such samples are necessarily easy to recruit, but because the researcher uses whatever individuals are available without incurring the cost or time required to select a random sample (Schaefer \& Dillman, 1998). Due to the personal nature of the responses, every effort to maintain confidentiality and anonymity was followed in this study. To maintain confidentiality, data was only accessed by the researcher. Maintaining anonymity was accomplished by excluding any questions that revealed identifying information about teachers. The pool of possible participants was generated from school staff rosters acquired from school principals or their designees (Chief Information Officers) that denote to which school a teacher is assigned. Generating the pool of participants in this manner decreased the chance that a participant, who works in more than one school, was e-mailed more than one invitation to participate. 


\section{School District Study Support}

To gain school district participation, district superintendents were contacted first by e-mail and then by a follow-up personal phone call requesting (a) permission to pursue survey research in each superintendent's respective districts and (b) to request a letter of support to local school principals (APPENDIX M). After superintendents gave permission for the study and expressed their support to local school principals, all school principals were contacted by e-mail for collaboration in this study within their respective schools (APPENDIX N). With the collaboration of school principals, the researcher requested a teacher roster for each school and assigned random survey identification numbers coded for confidentiality. Teacher participants were contacted by a pre-notice email informing them of the study, purpose, researchers and significance (APPENDIX O). Three days afterward, a follow-up e-mail was sent to request their participation in an internet-based questionnaire (APPENDIX P).

\section{Survey Research}

Survey research, the method of gathering data from respondents thought to be representative of a specific population, uses an instrument composed of closed or openended items (questions). Surveying is perhaps the dominant form of data collection in the social sciences, providing for efficient collection of data over broad populations, amenable to administration in person, by telephone, and over the internet. Some forms of survey research by telephone or the internet may be completely automated (Dillman et al., 1998) and data that had once been collected by other survey modes is now being collected with Web surveys (Dillman \& Bowker, 2001). Dillman et al. (1998) and Zanutto (2001) explained the relationship between principles of design and traditional 
sources of error in sampling, coverage, measurement, and non-response. Suggestions for increasing the response return rate (Zanutto, 2001) are as follows:

- Use a cover letter with the questionnaire;

- Make the survey simple;

- Provide an estimated time that it will take to complete the survey; no longer than twenty minutes;

- Design the first question so it is interesting, easy to answer, and related to the topic of the survey;

- Express concern about privacy issues for the respondents and the data that is collected.

\section{Web-based Questionnaires}

Whether to implement a personal interview survey or an internet-based questionnaire relies largely on the target population in the research effort. Internet-based surveys are directed through e-mail contact to internet users. Dillman, Best \& Krueger (2002) report that surveying via the internet is rapidly gaining popularity for data collection and offers the possibility of very rapid surveying (Schaefer \& Dillman, 1998) at a substantially reduced cost, and increased respondent flexibility (Dillman et al., 1998). Internet-based surveys can be done faster than telephone surveys, especially for large samples, where the number of telephones and trained interviewers limit the number of completions per day. A survey posted on a controlled Web site can collect thousands of responses in just a few hours (Dillman, Phelps, Tortora, Swift, Kohrell, \& Berck, 2001). Further, once setup is completed, virtually no cost is associated with an internet-based survey. 


\section{Self-report Web-based Questionnaire Return Rates}

Research in e-mail, mail, telephone, and face-to-face interviewing has universally found that the biggest predictor of response rates is the number of times a respondent is contacted (Schaefer \& Dillman, 1998). The greater the number of attempts made to reach participants, the greater the chance of increasing returns rates. Schaefer \& Dillman (1998) reports the average response rate for e-mail surveys with a single contact is $28.5 \%$, compared with $41 \%$ for two contacts and $57 \%$ for three or more contacts. Thus, this finding leads to the assertion that multiple contacts are an effective practice for increasing response rates.

Collecting data by two different modes of communication, i.e., oral and visual, raises the concern about measurement differences (Dillman et al., 2001). However, Carini et al. (2001) demonstrated that the responses of first-year and senior college students to traditional paper surveys versus Web surveys did not show substantial differences even after controlling for student and school characteristics. In the Carini et al. (2001) study, data were collected using Dillman et al. (2001) tailored design method. The Ohio State University researchers collected the data using a mailed survey technique. The Ohio State University research response rate was $96 \%(N=22)$ on the pretest and $100 \%(N=23)$ on the posttest. The University of Illinois research team conducted the small survey using a mailed survey technique for the pretest and administering the posttest questionnaire in person at a student teaching seminar. The University of Illinois research response rate was $92 \%(N=12)$ on the pretest and posttest. 


\section{Web-based Questionnaire Design}

The first step in internet-based surveying is to utilize a reliable survey (Dillman, 2000). The internet-based questionnaire used in this study was composed of validated scales used in previous studies to explore sclect background demographics, school climate, school culture, teacher efficacy, collective efficacy, job satisfaction, and teacher intent to turnover. Taking the time to create a questionnaire that appears professional, more personalized, or more attractive will have some positive effect on response rates (Fowler, 1993). The instrument should be easy to complete; task should be clear; questions should be attractively spaced, easy to read, and uncluttered; and the response task itself should include a check box, circle a number, or some other equally simple task. As recommended by Schafer \& Dillman (2002), a pilot study was conducted using twenty (20) subject matter experts to evaluate the content validity of the questionnaire.

Dillman et al. (1998) suggests that personalization is an important element in increasing response rates in mail surveys. When prospective respondents receive personalized letters addressed to them, they tend to feel important. This personalization technique can be applied to both e-mail and web-based questionnaires. This information is visible when the potential respondents open e-mail messages and see their own name as a salutation on a personal letter rather than listserv designations. An added benefit to personalized e-mail messages is that respondents are prevented from responding to other recipients of the survey, thus maintaining confidentiality.

Although interviews and observations could have provided rich and expansive data for use in research, this study used an e-mail request for participation in an internetbased questionnaire that had a uniform resource locator (URL) embedded in the message. 
The specifically targeted respondents simply clicked on this hypertext link, which then evoked their web browser, and presented the reader with the internet-based questionnaire. The internet-based questionnaire was electronically completed by the respondents in one twenty-minute session (Zanutto, 2001). The use of e-mail surveys has been restricted by the tendency of researchers to apply it only to such populations with nearly universal email access (Best \& Krueger, 2002).

\section{Web-based Questionnaire Data Collection}

The risk of non-coverage error, those who do not have access to e-mail and/or the internet, has prevented researchers from applying an e-mail method to other groups (Dillman, 2000; Dillman et al., 1998). However, an e-mail method with an internet-based questionnaire was utilized in this research because potential respondents for this study had universal e-mail and internet access provided to all Kentucky teachers through the Kentucky Educational Technology System.

Survey response rate is always a major concern when performing any research soliciting information from participants. Response rates for all types of surveys have been on the decline since 1990 (Dillman et al., 2001). Although response rates for Web surveys tend to be lower than mail surveys, Dillman reported several ways to improve web-based questionnaire response rates which have been verified in research studies. Solomon (2001) stated that personalized e-mail cover letters, follow-up reminders by email, pre-notification of the intent of the survey, simpler formats, and plain design have all been shown to improve response rates for Web-based surveys. The relationship the researcher or organization has with potential respondents may dramatically influence the response rate. Because all possible participants in this study work within YRE school 
calendars, respondents may have tended to get more involved with an issue to which they feel strongly, either positively or negatively (Solomon, 2001).

\section{Rationale for Measures}

Cronbach \& Shavelson (2004) report that rreliability is an essential characteristic of a good test, because if a test does not measure consistently (reliably), then one could not count on the scores resulting from a particular administration to be an accurate index of teacher's attitudes and beliefs. The alpha formula is one of several analyses that may be used to gauge the reliability (i.e., accuracy) of psychological and educational measurements (Cronbach et al., 2004). The most common test of reliability is Cronbach's alpha (i.e. "the reliability coefficient"), popularized in a 1951 article by Cronbach based on work in the 1940s by Guttman and others.

The variables of interest were listed in Table 1 along with the selected instruments and subscales. The variables were as follows: school climate, school culture, teacher efficacy, collective efficacy, teacher job satisfaction, and teacher intent to turnover. Due to interest in predicting the relationship of school climate, school culture, teacher efficacy, collective efficacy, and teacher job satisfaction on teacher's intent to leave the profession or transfer to other schools, intent to turnover was designated as the dependent variable. The other remaining variables were designated as independent variables. 


\section{Table 1}

Study Variables, Measures and Subscales

\begin{tabular}{|c|c|c|}
\hline Variables & Measures & Measure Subscales \\
\hline Dependent & Measures & Measure Subscales \\
\hline $\begin{array}{l}\text { Teacher Intent to } \\
\text { Turnover: }\end{array}$ & $\begin{array}{l}\text { Michigan Organizational } \\
\text { Assessment } \\
\text { Questionnaire }\end{array}$ & intent to turnover \\
\hline \multicolumn{3}{|l|}{ Independent } \\
\hline Background Data & $\begin{array}{l}\text { Background Information } \\
\text { Sheet }\end{array}$ & $\begin{array}{l}\text { ethnicity, gender, age, number of } \\
\text { years YRE teaching experience }\end{array}$ \\
\hline School Climate & $\begin{array}{l}\text { Organizational Climate } \\
\text { Index }\end{array}$ & $\begin{array}{l}\text { collegial leadership } \\
\text { professional teacher behavior } \\
\text { achievement press } \\
\text { institutional vulnerability }\end{array}$ \\
\hline School Culture & $\begin{array}{l}\text { School Culture Indicators } \\
\text { Scale }\end{array}$ & $\begin{array}{l}\text { inquiry practices } \\
\text { teacher learning community } \\
\text { collective problem solving }\end{array}$ \\
\hline Teacher Efficacy & $\begin{array}{l}\text { Teacher Sense of } \\
\text { Efficacy Scale }\end{array}$ & $\begin{array}{l}\text { efficacy in student engagement } \\
\text { instructional strategies } \\
\text { classroom management }\end{array}$ \\
\hline Collective efficacy & $\begin{array}{l}\text { Collective Teacher } \\
\text { Efficacy Scale }\end{array}$ & $\begin{array}{l}\text { task analysis } \\
\text { general competence }\end{array}$ \\
\hline $\begin{array}{l}\text { Teacher Job } \\
\text { Satisfaction }\end{array}$ & $\begin{array}{l}\text { Teacher Job Satisfaction } \\
\text { Scale }\end{array}$ & $\begin{array}{l}\text { global job satisfaction } \\
\text { teacher YRE satisfaction }\end{array}$ \\
\hline
\end{tabular}

School Climate. The Organizational Climate Index (OCI) is an organizational climate descriptive measure for schools. Building on early organizational climate research such as pioneers Halpin \& Croft (1963), who developed the Organizational Climate Description Questionnaire (OCDQ), Hoy, Smith \& Sweetland developed the 
$O C I$. The measure is a combination of the Organizational Health Index and Organizational Climate Descriptive Questionnaire (OCDQ). The OCI is a revision of the earlier School Climate Index. The OCI has four subscales - 1) collegial leadership (7 items, e.g. - "The principal explores all sides of the topics and admits that other opinions exist.") 2) professional teacher behavior (7 items, e.g. - "Teachers help and support each other.") 3) achievement press (8 items, e.g.-"The school set high standards for academic performance.") and, 4) institutional vulnerability (5 items, e.g.- "A few vocal parents can change school policy.") All participants were asked to rate each item on a four-point Likert-type scale ranging from: (1) "Very Frequently Occurs" to (4) "Rarely Occurs." Items 4, 14 and 30 are filler items and are not scored in the analysis. Lower rating scores reflect higher levels of school climate. Higher rating scores reflect lesser levels of school climate. Hoy et al. (2001) reported alpha coefficients of .94 (collegial principal behavior), .88 (professional teacher behavior), .92 (achievement press), and .87 (institutional vulnerability), respectively.

School Culture Indicators. The School Culture Indicators Scale is measure of school culture indicators by McLaughlin \& Talbert (2003) that explores the subscales of inquiry practice, teacher learning community and collective problem solving. The School Culture Indictors scale was designed for assessment for Reforming districts: How districts support school reform: A research report by McLaughlin \& Talbert (2003). The scale has three subscales: inquiry practices (5 items, e.g. - "My school has a clear vision of reform that is linked to standards for student learning and growth".), teacher learning community (4 items, e.g. - "I feel supported by colleagues to try out new ideas.") and collective problem solving (4 items, e.g. - "In this school we take steps to solve problems, 
we don't just talk about them."). All participants were asked to rate each item on a fivepoint Likert-type scale ranging from: (1) "Strongly Agree" to (5) "Strongly Disagree." Lower rating scores reflect higher levels of school culture. Higher rating scores reflect lesser levels of school culture. The character and strength of teachers' professional community within the school emerges as a significant factor in most all accounts of educational improvement (Riffle, Howley \& Ermolov, 2004). The coefficients of reliability for the School Culture Indicators Scale subscales are .86 (inquiry practice), .84 (teacher learning community), and .83 (collective problem solving), respectively.

Teacher Sense of Efficacy. The Teachers' Sense of Teacher Efficacy Scale was developed by Tschannen-Moran \& Woolfolk Hoy (2001). The researchers found three moderately correlated factors: efficacy in student engagement, efficacy in instructional practices, and efficacy in classroom management, but at times the researchers reported the make up of the scales varies slightly. The scale uses three subscales: 1) efficacy in student engagement (4 items, e.g. - "How much can you do to motivate students who show low interest in school work?") 2) instructional strategies (4 items, e.g. - "To what extent can you craft good questions for your students?"), and 3) Classroom Management (4 items, e.g. - "How much can you do to control disruptive behavior in the classroom?") Participants were directed to indicate on the questionnaire the extent to which they endorsed each statement. The scale used score of "1" for an item that means "A Great Deal", a score of "3" means "Quite a Bit", a score of "5" means "Some Influence", a score of "7" means "Very Little" and a score of "9" means "Nothing." Lower rating scores reflect higher levels of teacher efficacy. Higher rating scores reflect lesser levels of teacher efficacy. Between each scale unit there existed a partial rating for further 
clarification of degree of agreement. The reported reliabilities of the subscales were .81 (efficacy in student engagement), .86 (efficacy in instructional practices), and .86 (efficacy in classroom management).

Collective Efficacy. The Collective Teacher Efficacy Scale created by Goddard (2002a) was developed initially by modifying items from the original Gibson and Dembo (1984) teacher efficacy scale to reflect collective efficacy (i.e., changing the object of the efficacy items from "'l" to "We"(Goddard, 2002). Added items were written in response to a review by a panel of experts with experience in teacher efficacy research. The Collective Efficacy Scale has two dimensions: general competence (6 items, items 2, 4, and 5 are reverse of items 6, 9, and 21, e.g. - "Teachers in the school are able to get through to the most difficult students." and "If a child doesn't want to learn, teachers here give up.") and task analysis (6 items, items 12, 13, and 16 are reverse of items 14 , 19, and 20, e.g. - "These students come to school ready to learn." and "Students here just aren't motivated to learn."). All participants were asked to rate each item on a six point Likert-type scale ranging from (1) "Strongly Agree" to (6) "Strongly Disagree." Lower rating scores reflect higher levels of collective efficacy. Higher rating scores reflect lesser levels of collective efficacy. Criterion-related validity was assessed by correlating the scores from the 12-item instrument with those from the previous 21 -item instrument used in prior research on the construct (Goddard, 2001; Goddard et al., 2000). The results of this analysis yielded a high correlation $(r=.98)$ suggesting the shortened form was measuring the same construct as the longer form. In fact, the 12-item instrument was a true subset of the 21 -item instrument. Results from the pilot study suggested that the 12 items did indeed offer a valid and reliable measure of collective 
efficacy (Goddard, Hoy, Woolfolk \& Hoy, 2000). The alpha coefficient of reliability for the total scale was .96. A subsequent alpha correlation coefficient of .71, $(p<.001)$ showed there was a strong relationship between task analysis and group competence. An additional analysis found all the items loaded on one factor but were separated enough to represent two differing dimensions. $51 \%$ of the variance of all the items could be explained by one factor, 17 items loaded between .71 and .87 on a lone factor while the additional four items loaded between .47 and .70 on this same factor, offering further support for that collective efficacy was one construct made up of two separate but related dimensions (Goddard and Hoy, 2001).

Job Satisfaction. The Job Satisfaction Survey was adapted from the Teacher Job Satisfaction Questionnaire (TJSQ; Lester, 1987). Lester (1987) explained that the TJSQ was designed to measure teacher job satisfaction using randomly selected elementary, junior high school, and senior high school teachers in New York City, Nassau, Suffolk, and Westchester Counties. The Teacher Job Satisfaction Measure for this study used seventeen-items to measure teacher job satisfaction. Factor analysis of the TJSQ yielded nine factors: 1) supervision (e.g., "I am satisfied with the support I receive at my school.") 2) colleagues (e.g., "I am satisfied with the way I am treated by the people with whom I work.") 3) working conditions (e.g., "I do not like the climate where I work.") 4) pay (e.g., "I am satisfied with my pay.") 5) responsibility (e.g., "All in all, I am satisfied with my job as a teacher.") 6) advancement (e.g., "I am not satisfied with the promotional opportunities where I work.") 8) security (e.g., “In general, I like working at my school.") and 9) recognition (e.g., "I am satisfied with the feedback I have received about my performance at my job."). The Teacher Job Satisfaction Measure had two 
subscales: teacher global satisfaction (13 items) and teacher YRE satisfaction (4 items). Following Boyles (1993), McFadden (2004), Polidor (1996) and Worthen \& Zsiray (1994), the final four questions of the Teacher Job Satisfaction Measure asked participants to rate their agreement on satisfaction with: teaching in the YRE calendar (e.g., "All in all, I am satisfied with teaching in the year-round education calendar."); with student achievement in the YRE calendar (e.g., "In general, I am satisfied with student achievement in the year-round calendar."); with instructional flexibility in the YRE calendar (e.g., "All in all, I am satisfied with instructional flexibility in the yearround calendar."); and with their teaching effectiveness in the YRE calendar (e.g., "Overall, I am satisfied with my teaching effectiveness in the year-round calendar."). The participants were asked to rate the items on five-point Likert-type scale ranging from (1) "Strongly Agree" to (5) "Strongly Disagree." The internal consistency of the scale scores was .93 .

Boyles (1993) used a survey previously pilot tested with YRE calendar teachers and traditional calendar teachers in the Henderson City Schools in North Carolina, 1992. Teachers reported on the item concerning satisfaction with teaching: 86\% of YRE teachers rated high satisfaction with teaching; $38 \%$ of teachers on the traditional calendar rated high satisfaction. On satisfaction with student achievement, YRE teachers rated high satisfaction at $64 \%$ and traditional calendar teacher rated high satisfaction at $21 \%$. On instructional flexibility, YRE teachers rated high satisfaction at $72 \%$ while traditional calendar teachers rated high satisfaction at $25 \%$. On satisfaction with their teaching effectiveness YRE teachers rated high satisfaction at $72 \%$ while traditional calendar teachers rated their high satisfaction at 46\% (Boyles, 1993). 
Intent to Turnover. The Intent to Turnover Measure, adapted from the Michigan Organizational Assessment Questionnaire (MOAQ; Cammann, Fichman, Jenkins, \& Klesh, 1983) measured teachers' intent to turnover from their jobs or school. The fouritem Intent to Turnover Measure, adapted from the Michigan Organizational Assessment Questionnaire (MOAQ; Cammann, Fichman, Jenkins, \& Klesh, 1983), measured teachers' intent to turnover. The MOAQ assessed the individual's intention to continue to be an organizational member and the individual's desire to move to another job in the same organization (in this study, desire to seek a job in a school district with a traditional calendar). The specific items are as follows: 1) "I will probably look for a new job in the next year."; 2) "I often think about quitting."; 3) "How likely is it that you could find a job with another employer with about the same pay and benefits you now have?"; and 4) slightly modified), "If I had the chance, I would take the same job with a traditional calendar school district." (Likert-type Scale ranging from (1) "Not Likely at All" to (7) "Extremely Likely". Teachers indicated the degree to which they thought about looking for a job in the next year or thought about quitting their job. Lower scores reflect lower levels of intent to turnover. Higher scores reflect greater levels of intent to turnover. Three items $(1,2$, and 3$)$ were taken directly from the MOAQ. The fourth item is a modification of the third MOAQ item for purposes of measuring teachers' intent to turnover in the YRE work environment ("If I had the chance, I would take a same job with a traditional calendar school district."). The MOAQ was designed to assess the attitudes and perceptions of organizational members about a wide range of organizational attributes. The four-item measure was used to assess teachers' overall commitment to their jobs. Assessing teachers' intent to turnover is important when considering teachers' 
intention to continue to work in an alternative calendar school, to seek transfer to a school within the school district that continues the traditional agrarian calendar or to seek employment in another school district or leave the field of education. The internal consistency of the scale scores was .77. Moynihan \& Pandey (2003) report the coefficient alpha as cited in Cook, Hepworth, Wall \& Warr (1981) is given as .87.

Background information. Zey-Ferrell (1982) reports that age, gender, and selected measures of professionalism are major predictors of intent to turnover. Participants were asked to report individual demographic and background variables on a nine-item background demographic scale. Based on previous research on intent to turnover, the following variables were important: ethnicity, age, gender, and years teaching experience in a YRE calendar school.

\section{Procedures}

\section{Pre-notice E-mail}

According to Schaefer \& Dillman (1998) an introductory pre-notice e-mail requesting support and written permission for this study was sent to the superintendents in the targeted school districts. The researcher informed the superintendents of the value the school-based and district data might have for future district/school planning purposes. The pre-notice e-mail (APPENDIX M) to the superintendent explained the focus of the study and the importance of having access to teachers who have had teaching experience on a YRE calendar. To enlist further support, the researcher asked the superintendents to send a letter or e-mail of support for the research to the principals and teachers in their respective districts. 
With the permission and support of the superintendents, the researcher sent a prenotice e-mail (APPENDIX N) to each school principal in the target school districts to discuss the internet study and requested their cooperation in the study. Respective teacher rosters identified all possible participants who were assigned to particular schools for the school year. To assure confidentiality of respondents in schools with fewer than ten teachers, the researcher re-assigned these teachers to al larger, same district, school placement pool.

The researcher used the Leedy \& Ormrod (2005) Survey Random Number Table from Practical Research: Planning and Design for participant identification numbers. Recognized district codes and school codes might be easily interpreted by participants and/or district personnel. To maintain the confidentiality of the participants, the researcher assigned a data submission number (DSN) for each participant by selecting at random a two-digit number for each of the nine participating school districts, for each of the participating school within the school districts and finally for each potential participating teacher within schools for research tracking purposes. The DSN made it almost impossible for anyone, other than the researcher, to identify participants, districts, or schools. The DSN was provided to each appropriate participant in the second emailing (APPENDIX P) with the invitation to participate in the study. The researcher used Microsoft Exchange e-mail program, the standard e-mail program throughout schools and districts in Kentucky. By using the global e-mail address book for all Kentucky schools, the researcher designed e-mail distribution lists for each school in the target school districts. Participants were instructed to record the six-digit survey identification number in the DSN field on the internet-based survey. Follow-up e-mails 
were sent to participants thanking them for participation and secondly, encouraging those who has not completed the internet-based survey, to do so. The following steps (See Appendix L) were guided by Dillman (2001):

Step 1: The first participant contact pre-notice e-mail was sent to each teacher in the target schools on a Tuesday. Research has demonstrated that Monday and Friday are not the best days for requesting e-mail responses (Dillman). The pre-notice introduced the researchers, described the study goals, explained their importance to the research, and how valuable the results might be for teachers, schools and school districts in other communities considering the change to an alternative calendar -- which encouraged them to respond (Dillman \& Others, 1998). The pre-notice e-mail prepared the teachers for a follow-up survey e-mail in three days (See APPENDIX O).

Step 2: After two days (Thursday) the survey request for participation e-mail was sent containing the consent to participate, their rights and their reasonable expectation for confidentiality, instructions on how to enter the survey identification number, an embedded internet link to the survey questionnaire, how to complete the rating of items in the questionnaire, and how to successfully submit their completed questionnaire. The participants were assured confidentiality that the likelihood of risk through participation is minimal. Additionally, the researcher explained that participants could stop at any time or omit any question that may cause them discomfort without penalty (See APPENDIX P).

Step 3: After four days (Tuesday) the researcher sent a third e-mail to thank target teachers for their cooperation in reading previous e-mail contacts, thank them for 
participating, and encouraged teachers who had not completed the surveys to complete and submit their responses to enrich the results of the study (APPENDIX T).

Step 4: The researcher analyzed the Questionnaire Download Database for completed questionnaire data and calculated the return rate.

Step 5: The researchers sent final thank-you e-mail notices to all participant teachers and encouraged teachers who had not completed the surveys to complete and submit their responses to enrich the results of the study (APPENDIX W).

Step 6: The researchers sent final thank-you e-mail notices to all principals for their support and assistance in the data collection phase and encouraged principals to encourage teachers who had not completed the surveys to complete and submit their responses to enrich the results of the study (APPENDIX V).

Step 7: The researchers sent final thank-you e-mail notices to all superintendents for their support and assistance in the data collection phase and encouraged superintendents to remind principals to encourage teachers who had not completed the surveys to complete and submit their responses to enrich the results of the study (APPENDIX U).

\section{Data Analyses}

Step 8: The researchers exported participants' responses data from the Microsoft Access database to a Microsoft Excel spreadsheet for importing into the Statistical Package for the Social Sciences (SPSS; Norusis, 2006), for data analyses.

Step 9: The researchers performed statistical analyses of data for entry in Chapter IV of this study. 


\section{Answering the Research Questions}

To answer the three research questions, statistical procedural steps were followed. To answer the first research question, Pearson's $r$ correlations were utilized to examine the strength and direction of relationships between school climate, school culture, teacher efficacy, collective efficacy, teacher job satisfaction, and intent to turnover. To answer the second research question, Pearson's $r$ correlations were used to investigate the strength and direction of relationships between teachers' length of employment in a YRE calendar and intent to turnover. Finally, to answer the third research question, hierarchical regression analysis was employed to test the conceptual model developed from theory and research to guide this study (Cohen \& Cohen, 1983). Guided by the aforementioned theory and research, the separate steps in the analysis were as follows: (1) background variables, (2) school climate and school culture, (3) teacher efficacy, (4) collective efficacy, and (5) job satisfaction; each predicting the dependent variable (intent to turnover). The hierarchical regression analysis demonstrated the unique contribution of each variable (expressed as $R^{2}$ ) to predicting teacher intent to turnover. 


\section{CHAPTER IV}

\section{RESULTS}

The current study examined the extent to which school climate, school culture, teacher efficacy, collective efficacy, and teacher job satisfaction are related to teacher intent to turnover in a year-round education (YRE) callendar school context. The results from the open-ended question were explored as well to help in the interpretation of the quantitative data. This chapter includes the results of the study obtained through the quantitative and qualitative analyses of the questionnaire data. The independent variables included the demographic variables (ethnicity, age, gender, number of years teaching experience in a YRE calendar), school climate, school culture, teacher efficacy, collective efficacy, and teacher job satisfaction.

The dependent variable was teacher intent to turnover. A hierarchical regression analysis was employed to find the unique variance explained by school climate, school culture, teacher efficacy, collective efficacy, teacher job satisfaction and teacher intent to turnover in the context of a year-round education (YRE) calendar school after statistically controlling for the demographic variables. Data analysis was performed by using the 15.0 Statistical Package for Social Sciences (SPSS).

The three research questions that guided this study were: 
1. To what extent do school climate, school culture, teacher efficacy, collective efficacy, teacher job satisfaction relate to teacher intent to turnover in the YRE context?

2. To what extent does teachers' length of employment in a YRE calendar relate to teacher intent to turnover?

3. After controlling for the background variables (i.e., gender, age, years of YRE teaching experience), what is the unique contribution of school climate, school culture, teacher efficacy, collective efficacy, teacher job satisfaction and intent to turnover?

The results are presented include descriptive statistics of the sample and demographic variables, reliability statistics for each measure, and description of data analysis as it relates to answering the research questions. The discussion and implications of these results are presented in Chapter $\mathrm{V}$.

\section{Descriptive Variables}

The participants in this research study were certified (preschool-12) teachers employed by nine Kentucky school districts operating on a YRE calendar. The internetbased questionnaire used in this study collected: (a) demographic characteristics of participants (ethnicity, age, gender, highest educational degree, school level placement, content area, number of years teaching experience, number of years teaching experience in a YRE calendar, and salary range), (b) respondent level of agreement with rated items on scales measuring (school climate, school culture, teacher efficacy, collective efficacy, teacher job satisfaction, and teacher intent to turnover) and (c) respondent comments to an open-ended statement ("For me, personally and professionally, the year-round education 
calendar has ...." The items in the demographic and background section were derived from the literature and were later validated by a content validity analysis. APPENDIX X summarizes the characteristics of the sample by school districts.

The variable data were coded for analyses. The data coding used was the following:

- Age $(1=21-29$ years; $2=30-39$ years; $3=40-49$ years; $4=50-59$ years; $5=60 \&$ greater years of age).

- Gender $(0=$ Female; 1 = Male $)$.

- Number of years YRE teaching experience $(1=1-3$ years; $2=4-6$ years; $3=7$ -9 years; $4=10-11$ years).

Of the 748 respondents, $592(79 \%)$ were female. The percentage of female respondents is consistent with similar studies involving teachers within $\mathrm{K}-12$ teaching environments (Hoy \& Woolfolk, 1993). Out of the 748 respondents, 28 (4\%) were African-American, $2(.3 \%)$ were Asian-American, 709 (95\%) were white/Caucasian, 2 (.3\%) were Hispanic-American, and $7(.9 \%)$ individuals either did not identify their ethnicity or selected a unique label for their ethnicity (e.g., Other Ethnicity). The percentages of Caucasian and African-American respondents are representative of most of the rural school districts in Kentucky (Kentucky State Department of Education, 2006).

Participants entered chronological age across five categories: 21-29 (19.6\%), 30$39(29.8 \%), 40-49(26.8 \%), 50-49(21.4 \%)$, or 60 and greater $(2.4 \%)$. Respondents were relatively evenly spread among four of the five age categories with the highest participant rate among the 31-39 age category. The smallest participant category was the 60 or greater age category with only $2.4 \%$ of the study total. 


\section{Questionnaire Distribution and Response Rate}

The questionnaire was sent to the potential respondents in the nine Kentucky school districts. Part I of the internet-based questionnaire asked participants to provide demographic (ethnicity, age, gender, years of experience in YRE school calendar). Part II of the questionnaire asked participants to rate items on two school context scales of school climate (30 items) and school culture (13 items). Ratings ranged from (1) Strongly Agree to (5) Strongly Discagree. Part III of the questionnaire asked teacher to rate items on four scales measuring teacher efficacy (12 items), collective efficacy (12 items), teacher job satisfaction (17 items), and teacher intent to turnover (4 items). On teacher efficacy, the ratings ranged from (1) for "A Great Deal" to (9) for "Nothing"; on collective efficacy and teacher job satisfaction, the rating ranged from (1) Strongly Agree to (5) Strongly Disagree. For teacher intent to turnover, ratings ranged from (1) for "Not at all Likely" to (7) for "Extremely Likely."

The internet-based questionnaire was delivered to 1,254 respondents. After following multiple email contacts (Schaefer \& Dillman, 1998), the study resulted in 748 useable surveys, with a response rate of $59.6 \%$. These response rates are consistent with previous similar research with Internet surveys (Simsek \& Veiga, 2001). Out of 748 useable surveys, 345 questionnaires were returned after the first invitation to participate email; 210 were returned after the second email invitation; 188 were returned after a third email invitation. Table 2 shows number of respondents and percentages from each participating school district after the first email invitation, second email invitation and third email invitation. The second and third email invitation yielded $55.6 \%$ of the total surveys for the study. The return rate was consistent with previous internet survey 
research (Schaefer \& Dillman, 1998). The $N$ increased from 345 surveys after the first email invitation to a total $N$ of 748 surveys after the second and third email invitations. In addition, results indicate that School District $\mathrm{C}$ and School District contributed $22.3 \%$ and $22.2 \%$ of the total surveys respectively. School District A and School District D followed with $18.3 \%$ and $16.7 \%$ respectively. Table 12 indicates that potential respondents in School Districts A, E, and G responded at a return rate over $90 \%$ with $99.3 \%, 94.7 \%$ and $91.7 \%$, respectively. School Districts H, C, D, C had return rates of $70.5 \%, 60.0 \%, 56.4 \%$, and $55.1 \%$.

\section{Table 2}

Study Response Rate by Participating School Districts across Multiple Email Invitations (Three) to Participate

\begin{tabular}{|c|r|r|r|r|r|r|r|r|}
\hline $\begin{array}{c}\text { School } \\
\text { Districts }\end{array}$ & $\begin{array}{c}\text { First } \\
\text { Email }\end{array}$ & $\begin{array}{c}\text { \% of } \\
\text { District } \\
\text { Total }\end{array}$ & $\begin{array}{c}\text { Second } \\
\text { Email }\end{array}$ & $\begin{array}{c}\text { \% of } \\
\text { District } \\
\text { Total }\end{array}$ & $\begin{array}{c}\text { Third } \\
\text { Email }\end{array}$ & $\begin{array}{c}\text { \% of } \\
\text { District } \\
\text { Total }\end{array}$ & $\begin{array}{c}\text { Total } \\
\text { Surveys }\end{array}$ & $\begin{array}{c}\text { \% of Total } \\
\text { Surveys } \\
\text { Submitted }\end{array}$ \\
\hline A & 69 & 50.7 & 42 & 30.9 & 25 & 18.4 & 136 & 18.3 \\
\hline B & 8 & 33.3 & 6 & 25.0 & 10 & 41.7 & 24 & 3.2 \\
\hline C & 91 & 54.8 & 35 & 21.1 & 40 & 24.1 & 166 & 22.3 \\
\hline D & 42 & 33.9 & 43 & 34.7 & 39 & 31.5 & 124 & 16.7 \\
\hline E & 7 & 38.9 & 5 & 27.8 & 6 & 33.3 & 18 & 2.4 \\
\hline F & 23 & 53.5 & 9 & 20.9 & 11 & 25.6 & 43 & 5.8 \\
\hline G & 10 & 30.3 & 8 & 24.2 & 15 & 45.5 & 33 & 4.4 \\
\hline H & 75 & 45.5 & 52 & 31.5 & 38 & 23.0 & 165 & 22.2 \\
\hline I & 20 & 58.8 & 10 & 29.4 & 4 & 11.8 & 34 & 4.6 \\
\hline Totals & 345 & 44.4 & 210 & 27.3 & 188 & 28.3 & 743 & 100.0 \\
\hline
\end{tabular}

Note. $\mathrm{N}=743$ (5) submitted surveys did not list a data submission number which was coded by participating districts.

Survey Instrument: A Study of the Relationships between School Climate, School Culture, Teacher Efficacy, Collective Efficacy, Teacher Job Satisfaction and Intent to Turnover in the Context of Year-Round Education Calendars. 
To test the three emailing survey waves of responses for possible systematic bias, a wave analysis using an ANOVA investigated possible differences in the three wave groups (Rogelberg \& Luong, 1998). A two-way between groups analysis was conducted to explore the impact of wave group on the dependent variable intent to turnover. Respondents were divided into three groups according to the emailing wave in which they responded (Wave Group 1 - responded after the invitation to participate emailing and before the first reminder emailing; Wave Group 2 - responded after the first reminder and before the third emailing; and Wave Group 3 - responded after the third emailing contact and before the web-based questionnaire access was removed). See Table 3. 


\section{Table 3}

Two-Way ANOVA between E-mail Wave Groups Analysis

\begin{tabular}{ccccc}
\hline $\begin{array}{r}\text { Sum of } \\
\text { Squares }\end{array}$ & $d f$ & $\begin{array}{r}\text { Mean } \\
\text { Square }\end{array}$ & F & Sig. \\
\hline
\end{tabular}

Climate Total

$\begin{array}{rrrrrr}\text { Between Groups } & 209.553 & 2 & 104.777 & .959 & .384 \\ \text { Within Groups } & 60750.189 & 556 & 109.263 & & \\ \text { Total } & 60959.742 & 558 & & & \end{array}$

Climate Total

$\begin{array}{rrrrrr}\text { Between Groups } & 83.804 & 2 & 41.902 & .503 & .605 \\ \text { Within Groups } & 60333.995 & 724 & 83.334 & & \\ \text { Total } & 60417.799 & 726 & & & \end{array}$

Teaching Efficacy Total

$\begin{array}{rrrrrr}\text { Between Groups } & 91.852 & 2 & 45.926 & .312 & .732 \\ \text { Within Groups } & 104993.674 & 714 & 147.050 & & \\ \text { Total } & 105085.526 & 716 & & & \end{array}$

Collective Efficacy Total

$\begin{array}{rrrrr}\text { Between Groups } & 131.727 & 2 & 65.864 & .841 \\ \text { Within Groups } & 55543.329 & 709 & 78.340 & \\ \text { Total } & 55675.056 & 711 & \end{array}$

Teacher YRE Satisfaction

$\begin{array}{rrrrrr}\text { Between Groups } & 19.711 & 2 & 9.856 & .841 & .432 \\ \text { Within Groups } & 8371.912 & 714 & 11.725 & & \\ \text { Total } & 8391.623 & 716 & & \end{array}$

ITT2items

$\begin{array}{rrrrrr}\text { Between Groups } & .858 & 2 & .429 & .055 & .946 \\ \text { Within Groups } & 5651.390 & 727 & 7.774 & & \\ \text { Total } & 5652.248 & 729 & & \end{array}$

$p s<.05$

There was no statistically significant main effect for email wave $F(2,724)<.97, p s>$ $.05)$ and the effect size was very small $\left(n^{2}=.00\right)$. Thus, there were no statistically significant differences on the research variables by email mailing. 


\section{Content Validity}

To establish content validity before issuing the research measure, a panel of 20 subject matter experts (SME) involved in public PK-12 education, educational consulting and researching/promoting Year-Round Education was asked to review the internet-based questionnaire to determine if the research measure's items were indeed appropriate for this study. The use of a SME to determine content validity of surveys follows validity research by Sireci \& Geisinger (1993). The SME panel included (4) Superintendents, (2) Central Office Directors, (11) Principals/Assistant Principals, (2) Educational Consultants and (1) National Association for Year-Round Education Director. The SME panel members were asked to take the web-based questionnaire and provide feedback on the research measure's directions, the ease or lack of ease using the online surveying instrument, how to analyze the appropriateness of survey questions, design, response ratings, and any other suggestions that map improve the functionality and participation rate of the questionnaire. The web-based questionnaire address/link was embedded in an e-mail message to each subject matter expert. The questionnaire was accompanied with detailed requests on how to analyze instrument design, items and participant response methods. Some of subject matter experts responded by e-mail; others called and gave their feedback. Overall, the SMEs indicated the web-questionnaire was well organized and possessed appropriate content validity.

Among the SME comments were several on how to improve the appearance of the research instrument. The SME suggested background, font and response box colors, using a consistent format and font along the whole questionnaire, and keeping the column headings at right centered. Another group of suggestions involved navigation through the 
multiple scales and are consistent with previous internet survey research (Dillman et al., 2001; Schafer \& Dillman, 2002; Solomon 2001). Some of the suggestions were as follows:

- Place a certain number of items per page - avoid participants having to scroll down for more items.

- Use a "Next" button at the bottom of the page to take participants to next page of items to avoid participants from having to scroll to next page.

- Use a "Go Back" button for participants who might want to review their responses.

- Make response agreement ratings consistent - positive to negative ratings from one scale to another.

- Change the selections for year of teaching experience and year of teaching experience in a YRE calendar to input box rather than toggle selection buttons.

Overall, the SME feedback was subsequently incorporated as appropriate to the revised version of the web-based questionnaire.

\section{Research Measure Reliability}

The purpose of any reliability analysis is to determine if items in each scale are measuring the same construct (Cronbach \& Shavelson, 2004). Internal consistency reliability coefficients were calculated for the six research scales: School Climate, School Culture, Teacher Efficacy, Collective Efficacy, Teacher Job Satisfaction, and Teacher Intent to Turnover and subsequent subscales. School climate was measured by the Organizational Climate Index (OCI; Hoy et al., 2002) and school culture was assessed by 
the School Culture Indicators Scale (SCI; McLaughlin \& Talbert, 2003). Teacher efficacy was measured by the Teachers' Sense of Teacher Efficacy Scale, (TSTES; TschannenMoran \& Woolfolk Hoy, 2001) and collective efficacy was assessed by the Collective Efficacy Scale (CES; Goddard, 2002a). Teacher job satisfaction scale items were adapted from the Teacher Job Satisfaction Questionnaire (TJSQ; Lester, 1987). Following Boyles (1993), the final four questions of the Job Satisfaction Measure were modified to ask participants to rate their satisfaction teaching in the YRE calendar. The teacher intent to turnover scale items were adapted from the Michigan Organizational Assessment Questionnaire (MOAQ; Cammann et al., 1983), a measure of intent to turnover from current jobs. The total scale Cronbach's alphas ranging from .67 to .93 is presented in Table 3.

Reliability coefficients were calculated also for the four school climate subscales (collegial leadership, professional teacher behavior, achievement press, institutional vulnerability); the three school culture subscales (inquiry practice, teacher learning community, collective problem solving); the three teacher efficacy subscales (efficacy in student engagement, efficacy in instructional practices, efficacy in classroom management); the two teacher Job Satisfaction subscales (teacher global satisfaction, teacher YRE satisfaction) and two intent to turnover scales (intent to turnover- three items, intent to turnover - two items). Coefficient alpha is extensively used in empirical research to estimate the reliability of a test consisting of parallel items (Bandura, 1996). The Cronbach's alphas for the school climate subscales were as follows, .90 for the collegial leadership, .90 for professional teacher behavior, .80 for achievement press, and .75 for institutional vulnerability. The Cronbach's alphas for the school culture subscales 
were as follows: .88 for inquiry practice, .83 for teacher learning community, and .88 for collective problem solving. Additionally, the Cronbach's alphas for the teacher efficacy subscales were as follows: .87 for efficacy in student engagement, .85 for efficacy in instructional practices, and .91 for efficacy in classroom management.

Cronbach's alphas for the collective efficacy subscales were calculated as follows: group competence .81 and task analysis .72 . The teacher job satisfaction subscale teacher global job satisfaction was .86, while the Cronbach's alpha for teacher YRE satisfaction was .93. As can be seen in Table 3, reliabilities met the minimum or higher level (.70) considered acceptable for social science research (Nunnally \& Bernstein, 1994; Tabachnick \& Fidell, 2007).

Reliability analysis of the Intent to Turnover Scale (four items) yielded a low Cronbach's alpha of .54. Further reliability analysis indicated that Item \#1 had little relationship with items $2-4$. Thus, item \# 1 was removed from the intent to turnover scale. The result was an increase of reliability of the Intent to Turnover - Three Item Scale to .64. Additional analysis showed that removing item \#4 would increase the Intent to Turnover - Two Item Scale reliability to a more acceptable .67, consistent with Cronbach $\&$ Shavelson (2004). For the purpose of this study, then, the Intent to Turnover - Two Item Scale with a Cronbach's alpha of .67 was used for the subsequent statistical analyses. 


\section{Table 3}

Cronbach's Alphas for Research Scales and Subscales

\begin{tabular}{|c|c|c|}
\hline Scale & Cronbach's Alpha & \# of Items \\
\hline School Climate Total Scale & .86 & 30 \\
\hline collegial leadership & .90 & 7 \\
\hline professional teacher behavior & .90 & 7 \\
\hline achievement press & .80 & 8 \\
\hline institutional vulnerability & .75 & 4 \\
\hline School Culture Total Scale & .93 & 13 \\
\hline inquiry practice & .88 & 5 \\
\hline teacher learning community & .83 & 4 \\
\hline collective problem solving & .88 & 4 \\
\hline Teacher Efficacy Total Scale & .93 & 12 \\
\hline efficacy in student engagement & .87 & 4 \\
\hline efficacy in instructional practices & .85 & 4 \\
\hline efficacy in classroom management & .91 & 4 \\
\hline Collective Elficacy Total Scale & .83 & 12 \\
\hline group competence & .81 & 7 \\
\hline task analysis & .72 & 5 \\
\hline Teacher Job Satisfaction Total Scale & .90 & 17 \\
\hline teacher global satisfaction & .89 & 13 \\
\hline teacher YRE satisfaction & .93 & 4 \\
\hline \multicolumn{3}{|l|}{ Intent to Turnover Total Scale } \\
\hline Intent to Turnover - Two Items & .67 & 2 \\
\hline Intent to Turnover - Three Items & .64 & 3 \\
\hline
\end{tabular}

\section{Intercorrelations among Demographic and Research Variables}

Table 4 presents the correlations among the demographic and research variables (school climate, school culture, teacher efficacy, collective efficacy, teacher job satisfaction, and teacher intent to turnover). Hinkle, Wiersma \& Jurs (1988) suggest that, 
when discussing the strength of a correlation, a good rule of thumb would be to use the following descriptors:

- " $r$ " ranging from zero to about 20 may be regarded as indicating no or negligible correlation.

- " $r$ " ranging from about .20 to .40 may be regarded as indicating a low degree of correlation.

- " $r$ " ranging from about .40 to .60 may be regarded as indicating a moderate degree of correlation.

- " $r$ " ranging from about 60 to .80 may be regarded as indicating a marked degree of correlation.

- " $r$ " ranging from about 80 to 1.00 may be regarded as indicating high or strong correlation.

\section{Ethnicity}

Correlation analysis indicated that ethnicity had negligible statistically significant correlations with any of the demographic or research variables $(r s<.07 ; p s>.05)$.

\section{Age}

Respondents' age had a marked degree of statistically significant positive correlation with years of teaching experience $(.75 ; p<.01)$, a moderate degree of correlation with salary $(.53 ; p<.01)$, years of experience teaching in a YRE school calendar $(.50 ; p<.01)$, and highest degree $(.45 ; p<.01)$. The correlations suggest that as the years of experience teaching, years experience in a YRE calendar, degree, and salary increase, so does the age of respondent, which is of course logical.

Age had negligible or low statistically significant negative correlations with school climate total $(-.13 ; p<.01)$, school culture $(-.15 ; p<.01)$, teacher efficacy total $(-$ $.08 ; p<.05)$, and collective efficacy total $(-.10 ; p<.05)$. Age had negligible statistically significant correlation with teacher job satisfaction total (but exhibited a relationship with global job satisfaction $-.08, p<.05$ ) or teacher intent to turnover. These correlations are 
consistent with other previous research on the relation of teacher age to school climate (e.g., Brown, 2001; Gaziel, 2004). The low degree of relation between age and job satisfaction is consistent with previous research on age's relationship to job satisfaction (Williamson, 2006). Overall, the older participants were more likely to perceive themselves as having higher teaching and collective efficacy and global job satisfaction. Further, the older participants were more likely to perceive that their school climate was one where positive professional teacher behavior and achievement press exist. Likewise, the older participants were more likely to perceive that their school culture was one where inquiry practice, a teaching learning community, and collective problem solving were present. Interestingly, respondent age had no significant correlation with the dependent variable, i.e., intent to turnover and was consistent with previous research on the relationship between age and teacher intent to turnover (Gaziel, 2004). 
Table 4

Means, Standard Deviations and Correlations of Demographic and Research Variables

\begin{tabular}{|c|c|c|c|c|c|}
\hline Variable & $M$ & $S D$ & Age & Gender & $\begin{array}{c}\text { Years } \\
\text { Experience } \\
\text { YRE }\end{array}$ \\
\hline \multicolumn{6}{|l|}{ Demographic Variables } \\
\hline Age & 2.57 & 1.101 & & & \\
\hline Gender & .20 & .401 & -.03 & & \\
\hline Years Experience YRE & 3.38 & 3.239 & $.50 * *$ & -.06 & \\
\hline \multicolumn{6}{|l|}{ School Climate Scale } \\
\hline Collegial Leadership & 12.483 & 4.682 & -.06 & .00 & -.04 \\
\hline Professional Teacher Behavior & 12.017 & 4.023 & $-.14 * *$ & $.12 * *$ & -.07 \\
\hline Achievement Press & 17.881 & 3.890 & $-.10^{*}$ & .10 & -.07 \\
\hline Institutional Vulnerability & 14.098 & 3.991 & .02 & .00 & .01 \\
\hline Climate Total & 56.560 & 10.452 & $-.13 * *$ & .08 & -.07 \\
\hline \multicolumn{6}{|l|}{ School Culture Scale } \\
\hline Inquiry Practice & 8.855 & 3.531 & $-.14 * *$ & $.07 *$ & $-.09 *$ \\
\hline Teaching Learning Community & 8.126 & 3.048 & $-.12 * *$ & .03 & -.04 \\
\hline Collective Problem Solving & 9.095 & 3.648 & $-.13 * *$ & .00 & -.05 \\
\hline Culture Total & 26.055 & 9.123 & $-.15^{* * *}$ & .05 & -.07 \\
\hline \multicolumn{6}{|l|}{ Teacher Efficacy Scale } \\
\hline Student Engagement & 11.154 & 5.301 & -.06 & $.17 * *$ & -.02 \\
\hline Instructional Practices & 8.261 & 3.822 & -.03 & $.09 *$ & -.06 \\
\hline Classroom Management & 8.868 & 4.734 & $-.11 * *$ & .06 & $-.09 *$ \\
\hline Teacher Efficacy Total & 28.283 & 12.115 & $-.08^{*}$ & $.12 * *$ & -.06 \\
\hline \multicolumn{6}{|l|}{ Collective Efficacy Scale } \\
\hline Group Competence & .000 & 1.000 & -.03 & $.11 * *$ & -.00 \\
\hline Task Analysis & .000 & 1.000 & $-.11 * *$ & .05 & -.07 \\
\hline Collective Efficacy Total & 32.634 & 8.849 & $-.33 * *$ & $.11^{* *}$ & -.06 \\
\hline \multicolumn{6}{|l|}{ Teacher Job Satisfaction Scale } \\
\hline Teacher Job Satisfaction Global & 25.644 & 8.137 & $-.08 *$ & -.01 & -.02 \\
\hline Teacher YRE Satisfaction & 7.146 & 3.423 & .05 & $.09 *$ & $-.08 *$ \\
\hline Teacher Satisfaction Total & 32.771 & 10.056 & -.05 & .02 & -.05 \\
\hline \multicolumn{6}{|l|}{ Teacher Intent to Turnover Scale } \\
\hline Intent to Turnover Two Items & 3.840 & 2.785 & -.02 & .02 & -.05 \\
\hline Note: $\quad \begin{array}{r}N=748 * \text { Correlation } \\
* \text { Correlation }\end{array}$ & $\begin{array}{l}\text { ignifical } \\
\text { ignifical }\end{array}$ & $\begin{array}{l}\text { t the } p< \\
\text { t the } p<\end{array}$ & $\begin{array}{l}.01 \text { leve } \\
.05 \text { leve }\end{array}$ & $\begin{array}{l}2 \text {-tailed) } \\
\text { 2-tailed) }\end{array}$ & \\
\hline
\end{tabular}




\section{Gender}

Gender demonstrated negligible statistically significant positive correlations with school teacher efficacy total $(.12 ; p<.05)$ and collective efficacy total $(.12 ; p<.05)$. No statistically significant correlation existed with climate total $(.08 ; p>.05)$, school culture total $(.05 ; p>.05)$, teacher job satisfaction total $(.02 ; p>.05)$, and intent to turnover $(.04$; $p>.05)$. Further examination of the subscale correlations suggest that gender exhibited negligible positive relationships with professional teacher behavior (school climate), inquiry practice (school culture), student engagement and instructional practices (teacher efficacy), and group competence (collective efficacy). These correlations suggest that although the correlations were weak, males were more likely to perceive a school climate with professional teacher behavior and a school culture of inquiry practice. In addition, the male teachers were more likely to perceive higher teaching efficacy with regards to school engagement and instructional practices. Finally, males were more likely to rate group competence (collective efficacy) higher, as well as their YRE job satisfaction.

\section{Years YRE Teaching Experience}

Years of YRE teaching experience demonstrated a moderate degree of statistically significant positive correlations with age $(.50 ; p<.05)$. These moderate statistically significant positive correlations suggest that respondents with more YRE teaching experience were more likely to have taught more, be older, and have higher salaries. . Those participants with greater YRE experience were more likely to perceive their school climate as one where inquiry practice exists, and perceive they had higher classroom management efficacy. Years of YRE teaching experience had negligible statistically significant correlations with school climate total, school culture total, teacher 
efficacy total, collective efficacy total, teacher job satisfaction total, and teacher intent to turnover.

\section{Answering the Research Questions}

To answer the three research questions, a number of statistical procedural steps were followed. To answer the first research question, Pearson's $r$ correlations were utilized to examine the strength and direction of relationships between school climate, school culture, teacher efficacy, collective efficacy, teacher job satisfaction, and intent to turnover. To answer the second research question, Pearson's $r$ correlations were used to investigate the strength and direction of relationships between teachers' length of employment in a YRE calendar and teacher intent to turnover. Finally, to answer the third research question, hicrarchical regression analysis was employed to test the conceptual model developed from theory and research to guide this study (Cohen \& Cohen, 1983). Guided by the aforementioned theory and research, the separate steps in the analysis were as follows: (1) background variables, (2) school climate and school culture, (3) teacher efficacy, (4) collective efficacy, and (5) job satisfaction; each predicting the dependent variable (intent to turnover). The hierarchical regression analysis demonstrates the unique contribution of each variable (expressed as $R^{2}$ ) to predicting teacher intent to turnover. Table 5 lists the research questions and statistical measures for answering the research questions. 


\section{Table 5}

$\underline{\text { Research Questions and Statistical Measures }}$

\begin{tabular}{llll}
\hline Questions & Variables & Survey Items & $\begin{array}{l}\text { Statistical } \\
\text { Procedures }\end{array}$ \\
\hline
\end{tabular}

To what extent do Predictor Variables:

school climate, school climate: $1-30$

school culture, teacher efficacy, collective efficacy, teacher job

- collegial leadership

$1,3,5,10,13,20,27$

Pearson's $r$

- professional teacher behavior

$8,18,21,23,25,28$, 29

satisfaction relate to teacher intent to

- achievement press turnover in the YRE context?

- institutional vulnerability

$7,11,15,16,17,19$, 22,24

$2,6,9,12,26$

$4,14,30$ are filler items

school culture: $\quad 1-13$

- inquiry practice $1-5$

- teaching learning community 6-9

- collective problem solving 10-13

teacher efficacy:

- student engagement

- instructional practices

- classroom management

1-12

$2,3,4,11$

$5,9,10,12$

$1,6,7,8$

collective efficacy:

1-13

- group competence

Reverse scoring for

$1,2,5$

$3,4,9$

- task analysis

$6,7,10$

Reverse scoring for

$8,11,12$

teacher job satisfaction

1-17

- teacher job satisfaction global

- teacher YRE satisfaction

14-17

DV: intent to turnover: 
Table 5 continued

\begin{tabular}{|c|c|c|c|}
\hline Questions & Variables & $\underline{\text { Survey Items }}$ & $\begin{array}{l}\text { Statistical } \\
\text { Procedures }\end{array}$ \\
\hline $\begin{array}{l}\text { To what extent } \\
\text { does teachers' } \\
\text { length of } \\
\text { employment in a } \\
\text { YRE calendar } \\
\text { relate to teacher } \\
\text { intent to turnover? }\end{array}$ & $\begin{array}{l}\text { Predictor Variables; } \\
\text { Teachers' length of employment } \\
\text { in YRE calendar; } \\
\text { teacher job satisfaction }\end{array}$ & $\begin{array}{l}\text { Demographic } \\
\text { Variables }\end{array}$ & $\begin{array}{l}\text { Pearson's } r \\
\text { correlations }\end{array}$ \\
\hline $\begin{array}{l}\text { After controlling } \\
\text { for the background } \\
\text { variables (i.e., } \\
\text { gender, age, years } \\
\text { of YRE teaching } \\
\text { experience), what } \\
\text { is the unique } \\
\text { contribution of } \\
\text { school climate, } \\
\text { school culture, } \\
\text { teacher efficacy, } \\
\text { collective efficacy, } \\
\text { teacher job } \\
\text { satisfaction and } \\
\text { intent to turnover? }\end{array}$ & $\begin{array}{l}\text { Predictor Variables: } \\
\text { Background variables: } \\
\text { - gender } \\
\text { - age } \\
\text { - years of YRE teaching } \\
\text { school climate: } \\
\text { - collegial leadership } \\
\text { - professional teacher behavior } \\
\text { - } \text { achievement press } \\
\text { - institutional vulnerability } \\
\text { - Filler items } \\
\text { school culture: } \\
\text { - inquiry practice } \\
\text { - teaching learning } \\
\text { - community } \\
\text { teallective problem solving } \\
\text { - } \quad \text { student engagement } \\
\text { - instructional practices } \\
\text { - classroom management } \\
\text { collective efficacy: } \\
\text { - group competence } \\
\text { - task analysis } \\
\text { Reverse scoring for }\end{array}$ & $\begin{array}{l}1-30 \\
1,3,5,10,13,20,27 \\
8,18,21,23,25,28, \\
29 \\
7,11,15,16,17,19 \\
22,24 \\
2,6,9,12,26 \\
4,14,30 \\
1-13 \\
1-5 \\
6-9 \\
10-13 \\
1-12 \\
2,3,4,11 \\
5,9,10,12 \\
1,6,7,8 \\
1-13 \\
1,2,5 \\
\text { Reverse scoring for } \\
3,4,9 \\
6,7,10 \\
8,11,12\end{array}$ & $\begin{array}{c}\text { Hierarchical } \\
\text { regression } \\
\text { analysis }\end{array}$ \\
\hline
\end{tabular}


teacher job satisfaction

- teacher job satisfaction global

- teacher YRE satisfaction

DV: intent to turnover:
$1-17$

$1-13$

14- 17

2,3

\section{Answering Research Question One}

To what extent do school climate, school culture, teacher efficacy, collective efficacy. teacher job satisfaction relate to teacher intent to turnover in the YRE context?

To answer the first research question, Pearson's $r$ correlation coefficients (Pearson's $r$ ) were used to examine the correlations between the independent variables and the dependent variable intent to turnover (Vogt, 1999). Correlations were statistically significant at the .05 and .01 levels (2-tailed). All assumptions of normality, linearity, and homogeneity were met. The means, standard deviations, and correlations among the variables are presented in APPENDIX Y. Overall, there were multiple study variables with positive statistically significant correlations with other study variables.

\section{School Climate}

Respondents rated school climate scale items on the questionnaire by selecting from the following ratings: 1 = "Very Frequently Occurs," 2 = "Often Occurs," $3=$ "Sometimes Occurs," and $4=$ "Rarely Occurs." Low scores on the school climate scale items indicated respondents' overall positive view of the climate of their schools. Higher rating scores indicated a less positive or negative view of school climate. During correlational analyses, the variable school climate total yielded low statistically significant positive correlations with intent to turnover $(.28 ; p<.0)$. This correlation is consistent with previous research on school climate and teacher intent to turnover 
(Adams, 2001; Turan, 1998). Importantly too, a positive school climate was related to lower intent to turnover among this study's participants.

\section{School Culture}

Respondents rated school climate scale items on the questionnaire by selecting from the following ratings: $1=$ "Strongly Agree," $2=$ "Agree," $3=$ "Undecided," $4=$ "Disagree," and 5 = "Strongly Disagree." Low scores on school culture scale items indicated respondents' overall positive view of the culture of their schools. Higher rating scores indicated a less positive or negative view of school culture. During correlational analysis, school culture total yielded low statistically significant positive correlations with teacher intent to turnover $(.31 ; p<.01)$. These correlations are consistent with previous research on school culture teacher intent to turnover (Reames \& Spencer, 1998). In sum, a positive school culture was positively associated with lower intent to turnover.

\section{Teacher Efficacy}

Respondents rated teacher efficacy scale items on the questionnaire by selecting from the following range of ratings: "A Great Deal," "Quite a Bit," "Some Influence," "Very Little," and "Nothing." Respondents rated what how much they can do on the teacher efficacy scale items 1 = "A Great Deal," through $9=$ "Nothing." Low scores on teacher efficacy scale items indicated respondents' overall positive view of their teacher efficacy with regards to how much they can do regarding student engagement, instructional practices, and classroom management. Higher rating scores indicated a lesser positive or negative view of their teacher efficacy. During correlational analysis, teacher efficacy total yielded strong to moderate statistically significant positive correlations with teacher intent to turnover $(.23 ; p<.01)$. This correlation is consistent 
with previous research on the relation of teacher efficacy and teacher intent to turnover (Dee, 2002; Hoy \& Woolfolk, 1990). Hoy \& Woolfolk (1990) reported that teachers with high personal efficacy have the ability to teach or to know how to adjust their teaching to reach all kids. On the other hand, teachers with low personal efficacy doubt their abilities to reach all kids and tend to have higher intent to turnover. Hongyun et al. (2005) found that teachers' self-efficacy significantly predicted teachers' job satisfaction, work devotion, internal motivation and the satisfaction of colleague relationship and teacher turnover tendency; however, there were school level variations among these effects. Dee (2002) reported that teachers enter work organizations with expectations and values, and if these expectations and values are met, they will likely remain a member of the organization. Further, those with higher teaching efficacy were more likely to demonstrate higher collective efficacy and job satisfaction, and were less likely intending to turnover.

\section{Collective Efficacy}

\section{Rationale for Two-Factor Collective Efficacy}

A Principal Components Analysis (PCA) with varimax rotation was performed. Using the Kaiser Criterion, where all factors with eigenvalues greater than one are retained and usually rotated for the final solution, only two factors were extracted, as shown in Table 6.

The items loaded similarly on two factors identified in Goddard et al. (2000) on the 21-item Teacher Collective Efficacy Scale. The two factors identified in Goddard et al. (2000) were group competence (items 1, 2, 3, 4, 5, 9, and 11) and task analysis (items $6,7,8,10,12)$. Based on item content, two conceptually distinct factors were identified: 
1 pertaining to group competence scale items ( 7 items) and 1 pertaining to task analysis (5 items). Two factors consistently explained the highest proportion of the variance.

These were group competence (34\%) and task analysis $(21 \%)$, respectively. This finding is similar to other factor-analytic research on measures of collective efficacy (e.g.

Goddard et al., 2000; Goddard, 2002a) where the authors claimed, in the interest of clarity and parsimony, that two collective efficacy factors would be sufficient to describe the collective efficacy construct. The two factors' factor scores were used for subsequent analyses in this study.

\section{Table 6}

\section{PCA with Varimax Rotation of the Collective Efficacy Scale}

\begin{tabular}{ccc}
\hline ITEMS & Factor 1 & Factor 2 \\
\hline CE01 & .558 & .449 \\
CE02 & .686 & .396 \\
CE03 & .740 & .163 \\
CE04 & .780 & -.177 \\
CE05 & .692 & .207 \\
CE06 & .168 & .767 \\
CE07 & -.111 & .775 \\
CE08 & .349 & .535 \\
CE09 & .679 & .058 \\
CE10 & .077 & .716 \\
CE11 & .467 & .114 \\
CE12 & .306 & .458 \\
$(N=715)$ & &
\end{tabular}


Respondents rated collective efficacy scale items on the questionnaire by selecting from the following range of ratings: $1-6$ with $1=$ "Strongly Agree" and $6=$ "Strongly Disagree." Low scores on collective efficacy scale items indicated respondents' overall positive view of their collective efficacy in their school regarding group competence and task analysis. Higher rating scores indicated a less positive or negative view of their collective efficacy. During correlational analysis, both collective efficacy factors yielded low statistically significant positive correlations with teacher intent to turnover. These correlations are consistent with previous research on the relationship between collective efficacy, and teacher intent to turnover (Mawhinney et al., 2005).

The results support Mawhinney et al. (2005) that reported collective efficacy and teaching experience were more strongly related to the level of organizational commitment and less to intent to turnover. The statistically significant positive correlations suggest that study respondents who had higher group competence and task analysis collective efficacy were less likely to intend to turnover.

\section{Teacher Job Satisfaction}

Respondents rated teacher job satisfaction scale items on the questionnaire by selecting from the following range of ratings: $1-5$ with $1=$ "Strongly Agree" and $5=$ "Strongly Disagree." Low scores on teacher job satisfaction scale items indicated respondents' overall positive view of their teacher job satisfaction in their job. Higher rating scores indicated a less positive or negative view of their teacher job satisfaction. During correlational analysis, teacher job satisfaction global yielded strong to moderate statistically significant positive correlations with intent to turnover $(.54 ; p<.01)$. This correlation is consistent with previous research on the relation of teacher job satisfaction 
and teacher intent to turnover (Smith \& Ingersoll, 2004). The correlation of school climate and YRE satisfaction is consistent with previous research on satisfaction with the YRE school calendar (Tucker, 2003).

The results of this moderate degree of statistically significant positive correlational analysis supports Smith \& Ingersoll (2004) that reported job satisfaction is the most significant predictor of new teacher retention. The moderate statistically significant positive correlations suggest that study respondents who possess high teaching and collective efficacy are more likely to be satisfied with their jobs as teachers, and less likely to turnover.

\section{Intent to Turnover}

Respondents rated teacher intent to turnover scale items on the questionnaire by selecting from the following range of ratings: "Not At All Likely," "Somewhat Likely," "Quite Likely," and "Extremely Likely." Respondents rated teacher intent turnover scale items on a numeric range using $1=$ "Not At All Likely" through $7=$ "Extremely Likely." Low scores on teacher intent to turnover scale items indicated respondents' overall positive view of their overall intent to remain in their current job and school. Higher rating scores indicated an overall lesser intent to remain in their current job and school. As mentioned previously, teacher intent to turnover yielded strong to moderate statistically significant positive correlations with teacher job satisfaction, school culture, collective efficacy, school climate, and teacher efficacy. These correlations are consistent with previous research on the relation of teacher intent to turnover and school climate (Turan, 1998); teacher intent to turnover and school culture (Reames \& Spencer, 1998); teacher intent to turnover and teacher efficacy (Dee, 2002; Hoy \& Woolfolk, 1990); 
teacher intent to turnover and collective efficacy (Mawhinney et al., 2005); and finally, teacher job satisfaction and teacher intent to turnover (Smith \& Ingersoll, 2004).

The results of this strong to moderate statistically significant positive correlational analysis support Turan (1998) who found the existence of a statistically significant relationship between overall organizational climate of schools and teachers' organizational commitment and low interest in intent to turnover.

Overall, the statistically significant positive correlations suggest that school climate and culture, high teaching and collective efficacy and job satisfaction are positively associated with intent to turnover. Teacher intent to turnover correlational results are consistent with previous research on the relation of teacher intent to turnover and school climate (Adams, 2001; Turan, 1998); teacher intent to turnover and school culture (Reames \& Spencer, 1998); teacher intent to turnover and teacher efficacy (Dee, 2002; Hoy \& Woolfolk, 1990); teacher intent to turnover and collective efficacy (Mawhinney et al., 2005); and finally, teacher job satisfaction and teacher intent to turnover (Smith \& Ingersoll, 2004). Low scores on teacher intent to turnover scale items indicated respondents' overall positive view of their overall intent to remain in their current job and school. Higher rating scores indicated an overall lesser intent to remain in their current job and school. During correlational analysis, teacher intent to turnover total yielded strong to moderate statistically significant positive correlations with teacher job satisfaction total $(.55 ; p<.01)$ school culture total $(.31 ; p<.01)$, collective efficacy total $(.29 ; p<.01)$, school climate total $(.28 ; p<.01)$, and teacher efficacy total $(.23 ; p<.01)$. Results from the correlational analysis of teacher intent to turnover and other research variables indicates that teachers in this study spend little time thinking about 
quitting, transferring from their YRE schools to traditional calendar schools, or leaving the profession for other employment. Schools can expect continuity in professional teacher behaviors, instructional practices, student engagement, and group competence. As respondents indicate as satisfaction with school climate, school culture, teaching efficacy, collective efficacy and job satisfaction, teacher intent to turnover lowers and respondents are less likely to intend to turnover.

\section{Answering Research Question Two}

To what extent does teachers' length of employment in a YRE calendar relate to teacher intent to turnover?

Research Question \#2 explored to what extent does teachers' length of employment in a YRE calendar relate to teacher intent to turnover. To answer the second research question, Pearson's $r$ correlations were used to investigate the strength and direction of relationships between teachers' length of employment in a YRE calendar and intent to turnover. The dependent variable of teacher intent to turnover was measured by two seven-point Likert scale items $(1=$ Not At All Likely to $7=$ Extremely Likely). The Cronbach's alpha for the teacher intent to turnover - two items measure was .67. Teachers' years of YRE experience (length of employment in a YRE calendar) was self reported in Part I of the study questionnaire - "How many years have you taught in a Year-round Education calendar?" For analysis, the reported years of experience were broken down into four experience categories $(1=1-3$ years experience, $2=4-6$ years of experience, $3=7$ - 9 years of experience, and $4=10-11$ years of experience). Table 8 shows the descriptive statistics for the YRE experience categories. 
Mean scores and correlations for the research variables of teachers' years of YRE experience and intent to turnover were computed. The findings for the variables are shown in Table 8. An examination of the results indicates that the mean score for years employment in a YRE calendar was $6.38(S D=3.239)$ and teacher intent to turnover $10.58(S D=4.633)$.

Table 7 shows that Pearson's $r$ statistical analyses yielded a negligible significant correlation between years YRE experience and intent to turnover. The lack of a relationship between years of YRE teaching experience and teacher intent to turnover suggests that, based on the respondents' perceptions, that teachers with fewer years of YRE experience are no more likely to have higher intent to turnover levels than do respondents who had more years of YRE teaching experience. This result is in contrast to the years of teaching experience results discussed previously (years of teaching experience demonstrates a weak relationship with global and total job satisfactiongreater experience was related to greater satisfaction (Kneese, 2000) and does not support the previous research on the relation of years of YRE teaching experience and teacher intent to turnover (Minnesota Department of Children, Families \& Learning, 1999).

\section{Table 7}

Means, Standard Deviations, and Correlations for Demographic Items with Teachers' Years of Employment in a YRE calendar and Teacher Job Satisfaction with Teacher Intent to Turnover

\begin{tabular}{lccc}
\hline Variable & M & SD & Years Experience in YRE \\
\hline Years Experience in YRE & 6.38 & 3.239 & -.05 \\
Teacher Intent to Turnover & 3.84 & 2.785 & -
\end{tabular}

Note: ** Correlation is significant at the $p<.01$ level (2-tailed)

* Correlation is significant at the $p<.05$ level (2-tailed) 


\section{Answering Research Question Three}

After controlling for the background variables (i.e., gender, age, years of YRE teaching experience), what is the unique contribution of school climate, school culture, teacher efficacy, collective efficacy, teacher job satisfaction and intent to turnover?

Based on theory and empirical research, hierarchical regression was used to test the Conceptual Framework Model for Teacher Intent to Turnover in a YRE calendar context. This statistical procedure was employed to determine how much variance in the dependent variable, intent to turnover, can be explained by a combination of independent variables (Sweet \& Grace-Martin, 2003). Theory and research determined the order of entry of the variables (i.e., blocks) into the regression equation. In this study, the first block consisted of the demographic variables. The second block consisted of school climate and school culture variables. The third block consisted of the teacher efficacy variables. The fourth block consisted of collective efficacy (group competence and task analysis) and the fifth block consisted of teacher job satisfaction (i.e., teacher global satisfaction-global satisfaction was selected to represent the satisfaction variable because it demonstrated the strongest correlations overall with the other research variables). The regression analyses were completed to examine the extent to which the combinations of demographic variables, school climate, school culture, teacher efficacy, collective efficacy, and teacher job satisfaction contributed unique variance to teacher intent to turnover.

The results of the hierarchical regression analysis for the Intent to Turnover Conceptual Model are presented in Table 8. With gender, age, ethnicity, and years experience in YRE in the first block of the regression equation, $1.0 \%$ of the variance 
was explained in the regression equation. Only years YRE experience reached statistical significance in the block.

After statistically controlling for the demographic variables, the addition of school climate subscales scores (collegial leadership, professional teacher behavior, achievement press, and institutional vulnerability), and school culture subscale scores (inquiry practice, teaching learning community and collective problem solving) into the second block of the regression equation resulted in an additional $14 \%(p<.001)$ of the variance being predicted in the regression equation; a large size effect (Cohen, 1988). Of the separate variables, school climate subscale scores (collegial leadership) and school culture subscale scores (inquiry practice and collective problem solving) positively contributed to the intent to turnover regression equation (less likely to turnover).

After statistically controlling for the demographic variables (gender, age and years experience in YRE, school climate subscales scores (collegial leadership, professional teacher behavior, achievement press), school culture subscales scores (institutional vulnerability, inquiry practice, teaching learning community and collective problem solving), the addition of teacher efficacy subscales scores (student engagement, teacher instructional practices and classroom management) into the third block of the regression equation resulted in an additional $2 \%(p=.01)$ of the variance being predicted in the regression equation. Only the teacher efficacy subscale student engagement variable contributed significantly to predicting the dependent variable.

After statistically controlling for the prior three blocks of variables, the addition of collective efficacy subscales scores (group competence and task analysis) into the fourth block of the regression equation resulted in an additional $1 \%(p=.05)$ of the variance 
being predicted in the regression equation. The collective efficacy subscale score (task analysis) was the only variable that contributed unique variance to the regression equation.

Finally, after statistically controlling for the prior four blocks of variables, the addition of teacher job satisfaction subscale scores (teacher global satisfaction) into the fifth block of the regression equation resulted in an additional 19\% of variance being predicted in the regression equation; a large size effect (Cohen, 1988). Overall, the regression model predicted $38.0 \%$ of the variance in teacher intent to turnover. It should be noted that these results were obtained for one particular order of entry of the independent variables, based on the conceptual model that guided this study presented in Figure 5 .

\section{Conceptual Model}

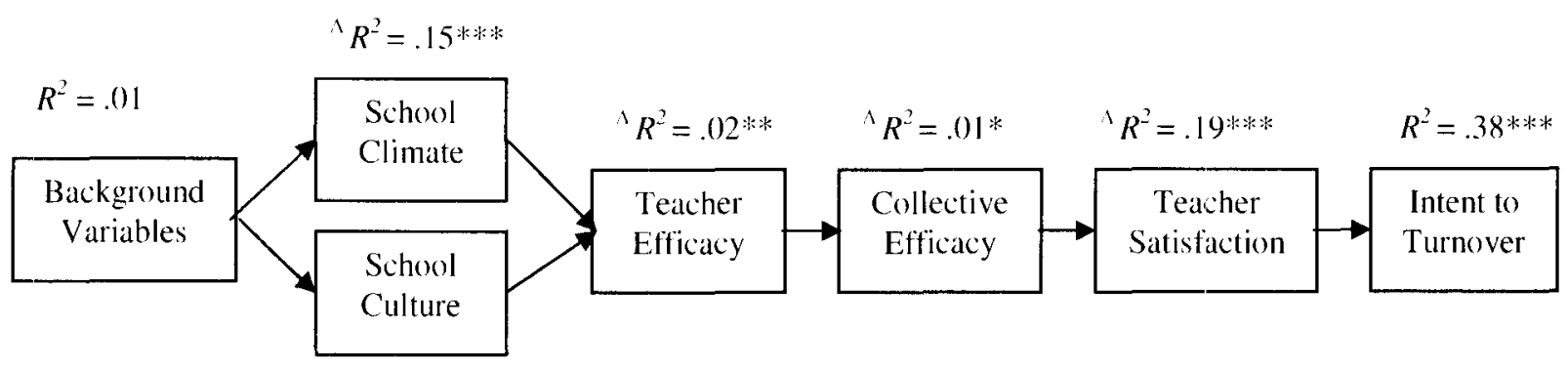

Figure 5. Conceptual Model for Teacher Intent to Turnover in a YRE Context 


\section{Table 8}

Summary of Hierarchical Regression Analysis for Variables Predicting

Teacher Intent to Turnover (Intent to Turnover) Predicted by five Variables

\begin{tabular}{|c|c|c|c|c|}
\hline Step and Predictor Variable & Beta & $R^{2}$ & ${ }^{\wedge} R^{2}$ & $p$ \\
\hline \multicolumn{5}{|l|}{ Step 1} \\
\hline $\begin{array}{l}\text { Age } \\
\text { Gender } \\
\text { Ethnicity } \\
\text { Years Experience } \\
\text { Years Experience YRE } \\
\text { Block }\end{array}$ & $\begin{array}{r}.03 \\
-.02 \\
-.02 \\
.08 \\
-.15\end{array}$ & .01 & .01 & $\begin{array}{l}.56 \\
.54 \\
.61 \\
.17 \\
.00 \\
.33\end{array}$ \\
\hline \multicolumn{5}{|l|}{ Step 2} \\
\hline $\begin{array}{l}\text { Climate-Collegial Leadership } \\
\text { Climate-Professional Teacher Behavior } \\
\text { Climate-Achievement Press } \\
\text { Climate-Institutional Vulnerability } \\
\text { Culture-Inquiry Practice } \\
\text { Culture-Teaching Learning Community } \\
\text { Culture-Collective Problem Solving } \\
\text { Block }\end{array}$ & $\begin{array}{r}-.12 \\
-.02 \\
.03 \\
.00 \\
-.12 \\
.14 \\
.03\end{array}$ & .16 & .15 & $\begin{array}{l}.03 \\
.78 \\
.59 \\
.99 \\
.05 \\
.05 \\
.67 \\
.00\end{array}$ \\
\hline \multicolumn{5}{|l|}{ Step 3} \\
\hline $\begin{array}{l}\text { Teacher Efficacy-Student Engagement } \\
\text { Teacher Efficacy-Instructional Practices } \\
\text { Teacher Efficacy-Classroom Management } \\
\text { Block }\end{array}$ & $\begin{array}{l}-.14 \\
-.07 \\
-.05\end{array}$ & .18 & .02 & $\begin{array}{l}.02 \\
.15 \\
.34 \\
.01\end{array}$ \\
\hline \multicolumn{5}{|l|}{ Step 4} \\
\hline $\begin{array}{l}\text { Collective Efficacy - Group Competence } \\
\text { Collective Efficacy - Task Analysis } \\
\text { Block }\end{array}$ & $\begin{array}{l}-.02 \\
-.06\end{array}$ & .19 & .01 & $\begin{array}{l}.69 \\
.04 \\
.05\end{array}$ \\
\hline \multicolumn{5}{|l|}{ Step 5} \\
\hline $\begin{array}{l}\text { Teacher Job Satisfaction-Teacher Global } \\
\text { Satisfaction } \\
\text { Block }\end{array}$ & -.65 & .38 & .19 & .00 \\
\hline $\begin{array}{l}\text { Total } R^{2} \\
\text { Note. } \\
N=472\end{array}$ & & .38 & .38 & .00 \\
\hline
\end{tabular}


The largest beta coefficient in Step 1 was $-.15(p<.05)$ which is for teachers' years of YRE teaching experience. This variable makes the strongest unique contribution to explain the dependent variable, when the variance explained by all the other variables in Step 1, are controlled. Analyzing Step 2, results indicate that (teaching learning community) school culture makes the strongest unique contribution to explain the dependent variable $(-.14, p<.05)$, followed by (collegial leadership) school climate and (inquiry practice) school culture. Teacher efficacy subscale (student engagement) made the strongest unique contribution $(-.14, p<.05)$ in Step 3. Step 4 results show that (task analysis) collective efficacy $(-.04, p<.05)$ makes the strongest predictors in Step 4 . The strongest unique contribution to explain the dependent variable intent to turnover was (teacher global satisfaction) teacher job satisfaction. Overall, the conceptual model tested in this study explained $38.0 \%$ of the variance in intent to turnover in a YRE context.

\section{Qualitative Data}

The qualitative part of the internet-based questionnaire was comprised of one open-ended question that participants answered pertaining to their perceptions of benefits of the YRE calendar, both personal and professional. Refer to Appendix Q for this survey question. Refer to Appendix $Z$ for responses to this survey question. The results from the open-ended question were examined to provide further depth and breadth to the quantitative research findings.

\section{"For me, personally and professionally, the year-round education calendar has ...."}

The open-ended question stem queried respondents about what they feel working in the YRE school calendar did for them from a personal and professional perspective.

Presented in Table 10 are the frequencies and percentages collected from respondents 
indications of the impacts of the YRE school calendar they found most beneficial to them.

Results of the open-ended survey question indicated that 562 of 748 respondents for this study chose to respond to the open-ended statement. 509 of the $562(90.6 \%)$ responded favorably toward YRE while $53(9.4 \%)$ responded negatively toward YRE in their comments. With further comment analysis, respondents' comments clustered around positive and negative comments in two categories. These categories are as follows:

Positive Comments

- Advantages for Teachers

- Advantages for Students
Negative Comments

- Disadvantages for Teachers

- Disadvantages for Students

All respondent comments were analyzed for positive and negative responses to the openended question. Comments that used positive descriptors (e.g. "like," "wonderful," "enjoy") were coded as a reported positive advantage for teachers or students. Comments that used negative descriptors (e.g. "do not like," "hate," "disadvantage") were coded as negative and as disadvantages for teachers and students. See Table 9 for presentation of the participant responses. 


\section{Table 9}

Frequencies and Percentages of Participants' Responses Regarding Teacher and Student Benefits Resulting from the YRE Calendar

Demographic Categories

\begin{tabular}{|c|c|c|c|c|c|}
\hline & $\begin{array}{r}\text { Total } \\
\text { Surveys } \\
\text { with } \\
\text { comments }\end{array}$ & $\begin{array}{l}\text { Total } \\
\text { Surveys } \\
\text { Comments } \\
\text { Positive } \\
\text { Toward } \\
\text { YRE }\end{array}$ & $\begin{array}{l}\text { Percent } \\
\text { Survey } \\
\text { Comments } \\
\text { Positive } \\
\text { Toward } \\
\text { YRE }\end{array}$ & $\begin{array}{r}\text { Total } \\
\text { Surveys } \\
\text { Comments } \\
\text { Negative } \\
\text { Toward } \\
\text { YRE } \\
\end{array}$ & $\begin{array}{r}\text { Percent } \\
\text { Survey } \\
\text { Comments } \\
\text { Negative } \\
\text { Toward } \\
\text { YRE }\end{array}$ \\
\hline \multicolumn{6}{|l|}{ Ethnicity } \\
\hline African-American & 19 & 18 & $94.7 \%$ & 1 & $5.3 \%$ \\
\hline Asian-American & 2 & 2 & $100.0 \%$ & 0 & $0.0 \%$ \\
\hline White/Caucasian & 534 & 482 & $90.3 \%$ & 52 & $9.7 \%$ \\
\hline Hispanic- & & 2 & & & \\
\hline American & 2 & & $100.0 \%$ & 0 & $0.0 \%$ \\
\hline Other & 3 & 3 & $100.0 \%$ & 0 & $0.0 \%$ \\
\hline Total & 560 & 507 & & 53 & \\
\hline Missing System & 2 & 2 & $100.0 \%$ & 0 & $0.0 \%$ \\
\hline Total & 562 & 509 & & 53 & \\
\hline \multicolumn{6}{|l|}{ Age Categories } \\
\hline $21-29$ & 103 & 97 & $94 \%$ & 6 & $6 \%$ \\
\hline $30-39$ & 183 & 166 & $91 \%$ & 16 & $9 \%$ \\
\hline $40-49$ & 148 & 132 & $89 \%$ & 17 & $11 \%$ \\
\hline $50-59$ & 115 & 102 & $89 \%$ & 13 & $11 \%$ \\
\hline 60 and greater & 13 & 12 & $92 \%$ & 1 & $8 \%$ \\
\hline Total & 562 & 509 & & 53 & \\
\hline Missing System & 0 & 0 & $0.0 \%$ & 0 & $0.0 \%$ \\
\hline Total & 562 & 509 & & 53 & \\
\hline \multicolumn{6}{|l|}{ Gender } \\
\hline Female & 444 & 402 & $90.5 \%$ & 41 & $9.5 \%$ \\
\hline Male & 116 & 105 & $90.5 \%$ & 12 & $10.5 \%$ \\
\hline Total & 560 & 507 & & 53 & \\
\hline Missing System & 2 & 2 & $100.0 \%$ & 0 & $0.0 \%$ \\
\hline Total & 562 & 509 & & 53 & \\
\hline
\end{tabular}




\begin{tabular}{|c|c|c|c|c|c|}
\hline & $\begin{array}{r}\text { Total } \\
\text { Surveys } \\
\text { with } \\
\text { comments }\end{array}$ & $\begin{array}{l}\text { Total } \\
\text { Surveys } \\
\text { Comments } \\
\text { Positive } \\
\text { Toward } \\
\text { YRE }\end{array}$ & $\begin{array}{l}\text { Percent } \\
\text { Survey } \\
\text { Comments } \\
\text { Positive } \\
\text { Toward } \\
\text { YRE }\end{array}$ & $\begin{array}{r}\text { Total } \\
\text { Surveys } \\
\text { Comments } \\
\text { Negative } \\
\text { Toward } \\
\text { YRE }\end{array}$ & $\begin{array}{r}\text { Percent } \\
\text { Survey } \\
\text { Comments } \\
\text { Negative } \\
\text { Toward } \\
\text { YRE }\end{array}$ \\
\hline \multicolumn{6}{|l|}{ Age Categories } \\
\hline $21-29$ & 103 & 97 & $94.2 \%$ & 6 & $5.8 \%$ \\
\hline $30-39$ & 183 & 166 & $90.7 \%$ & 16 & $8.7 \%$ \\
\hline $40-49$ & 148 & 132 & $89.2 \%$ & 17 & $11.5 \%$ \\
\hline $50-59$ & 115 & 102 & $88.7 \%$ & 13 & $11.3 \%$ \\
\hline 60 or Greater & 13 & 12 & $92.3 \%$ & 1 & $7.7 \%$ \\
\hline Total & 562 & 509 & & 53 & \\
\hline Missing System & 0 & 0 & $0.0 \%$ & 0 & $0.0 \%$ \\
\hline Total & 562 & 509 & & 53 & \\
\hline \multicolumn{6}{|c|}{ Highest Educational Degree } \\
\hline Bachelors Degree & 95 & 84 & $88.4 \%$ & 11 & $11.6 \%$ \\
\hline Masters Degree & 285 & 257 & $90.2 \%$ & 28 & $9.8 \%$ \\
\hline Rank I Degree & 175 & 161 & $92.0 \%$ & 14 & $8.0 \%$ \\
\hline Doctoral Degree & 5 & 5 & $100.0 \%$ & 0 & $0.0 \%$ \\
\hline Total & 560 & 507 & & 53 & \\
\hline Missing System & 2 & 2 & $0.0 \%$ & 0 & $0.0 \%$ \\
\hline Total & 562 & 509 & & 53 & \\
\hline \multicolumn{6}{|c|}{ School Level Placement } \\
\hline $\begin{array}{l}\text { Preschool } \\
\text { Elementary }\end{array}$ & 7 & 7 & $100.0 \%$ & 0 & $0.0 \%$ \\
\hline School & 285 & 265 & $93.0 \%$ & 20 & $7.0 \%$ \\
\hline Middle School & 99 & 91 & $91.9 \%$ & 8 & $8.1 \%$ \\
\hline High School & 169 & 144 & $85.2 \%$ & 25 & $14.8 \%$ \\
\hline Total & 560 & 507 & & 53 & \\
\hline Missing System & 2 & 2 & $0.0 \%$ & 0 & $0.0 \%$ \\
\hline Total & 562 & 509 & & 53 & \\
\hline
\end{tabular}




\begin{tabular}{|c|c|c|c|c|c|}
\hline & $\begin{array}{r}\text { Total } \\
\text { Surveys } \\
\text { with } \\
\text { comments }\end{array}$ & $\begin{array}{l}\text { Total } \\
\text { Surveys } \\
\text { Comments } \\
\text { Positive } \\
\text { Toward } \\
\text { YRE }\end{array}$ & $\begin{array}{l}\text { Percent } \\
\text { Survey } \\
\text { Comments } \\
\text { Positive } \\
\text { Toward } \\
\text { YRE }\end{array}$ & $\begin{array}{r}\text { Total } \\
\text { Surveys } \\
\text { Comments } \\
\text { Negative } \\
\text { Toward } \\
\text { YRE }\end{array}$ & $\begin{array}{r}\text { Percent } \\
\text { Survey } \\
\text { Comments } \\
\text { Negative } \\
\text { Toward } \\
\text { YRE }\end{array}$ \\
\hline \multicolumn{6}{|c|}{ Content Instructional Area } \\
\hline $\begin{array}{l}\text { Self-Contained } \\
\text { Classroom } \\
\text { Reading }\end{array}$ & 227 & 165 & $92.7 \%$ & 12 & $7 \%$ \\
\hline $\begin{array}{l}\text { Instruction } \\
\text { Language Arts }\end{array}$ & 54 & 41 & $82.9 \%$ & 7 & $17 \%$ \\
\hline $\begin{array}{l}\text { Instruction } \\
\text { Mathematics }\end{array}$ & 53 & 44 & $88.6 \%$ & 5 & $11 \%$ \\
\hline $\begin{array}{l}\text { Instruction } \\
\text { Science }\end{array}$ & 58 & 48 & $91.7 \%$ & 4 & $8 \%$ \\
\hline $\begin{array}{l}\text { Instruction } \\
\text { Social Studies }\end{array}$ & 72 & 53 & $88.7 \%$ & 6 & $11 \%$ \\
\hline $\begin{array}{l}\text { Instruction } \\
\text { Arts \& } \\
\text { Humanities }\end{array}$ & 60 & 48 & $91.7 \%$ & 4 & $8 \%$ \\
\hline $\begin{array}{l}\text { Instruction } \\
\text { Practical Living }\end{array}$ & 29 & 24 & $87.5 \%$ & 3 & $13 \%$ \\
\hline Instruction & 37 & 29 & $86.2 \%$ & 4 & $14 \%$ \\
\hline Other Instruction & 151 & 107 & $92.5 \%$ & 8 & $7 \%$ \\
\hline Total & 741 & 559 & & 53 & \\
\hline Missing System & 7 & 3 & $100.0 \%$ & 0 & $0.0 \%$ \\
\hline Total & 748 & 562 & & 53 & \\
\hline \multicolumn{6}{|c|}{ Years of Teaching Experience } \\
\hline 1- 5 Years & 133 & 122 & $91.7 \%$ & 11 & $8.3 \%$ \\
\hline $6-10$ Years & 128 & 116 & $90.6 \%$ & 12 & $9.4 \%$ \\
\hline 11-15 Years & 94 & 83 & $88.3 \%$ & 11 & $11.7 \%$ \\
\hline $16-20$ Years & 65 & 59 & $90.8 \%$ & 6 & $9.2 \%$ \\
\hline $21-25$ Years & 46 & 40 & $87.0 \%$ & 6 & $13.0 \%$ \\
\hline $\begin{array}{l}26-30 \text { Years } \\
30 \text { and Greater }\end{array}$ & 49 & 46 & $93.9 \%$ & 3 & $6.1 \%$ \\
\hline Years & 29 & 26 & $89.7 \%$ & 3 & $10.3 \%$ \\
\hline Total & 544 & 17 & & 52 & \\
\hline Missing System & 18 & 17 & $94.4 \%$ & 1 & $5.6 \%$ \\
\hline Total & 562 & 509 & & 53 & \\
\hline
\end{tabular}


Table 9, continued

\begin{tabular}{rllrr}
\hline Total & Total & Percent & Total & Percent \\
Surveys & Surveys & Survey & Surveys & Survey \\
with & Comments & Comments & Comments & Comments \\
comments & Positive & Positive & Negative & Negative \\
& Toward & Toward & Toward & Toward \\
& YRE & YRE & YRE & YRE \\
\hline
\end{tabular}

Years of Teaching Experience in YRE

$1-3$ Years of

YRE

Experience

4-6 Years of

139

123

$88.5 \%$

16

$11.5 \%$

YRE

Experience

$7-9$ Years of

114

110

$96.5 \%$

4

$3.5 \%$

YRE

Experience

164

147

$89.6 \%$

17

$10.4 \%$

10 - 11 Years

of YRE

Experience

128

113

$88.3 \%$

15

$88.5 \%$

Total

493

$88.9 \%$

51

Missing

System

18

16

2

Total

562

509

$11.1 \%$

53

Salary Range

$\$ 20,001$ -

$\$ 30,000$

23

18

$78.3 \%$

5

$21.7 \%$

$\$ 30,001$ -

$\$ 40,000$

174

161

$92.5 \%$

13

$7.5 \%$

$\$ 40,001$ -

$\$ 50,000$

216

197

$91.2 \%$

19

$8.8 \%$

$\$ 50,001$ -

$\$ 60,000$

122

109

$89.3 \%$

13

$10.7 \%$

$\$ 60,001$ -

$\$ 70,000$

$\$ 70,000 \&>$

17

Total

14

$82.4 \%$

3

$17.6 \%$

$3 \quad 100.0 \%$

0

$0.0 \%$

555

502

53

$98.5 \%$

Missing

System

Total

7

7

$100.0 \%$

$0.0 \%$

562

509

0

53 
Initially, the most notable observation of participant responses was the percentage of all participants who chose to respond (75\%) to the open-ended statement. Participant responses ranged one-word responses like "Wonderful" to more detailed responses that list personal and professional benefits from the YRE calendar. The length, specificity, and details found in the responses indicated that there may be some differences in the level of advocacy for the YRE calendar across schools, districts, and teacher demographics, at the very least a difference in the enthusiasm level of teachers concerning the YRE calendar. Results indicated that 509 of the $562(90.6 \%)$ participants who responded to the qualitative question reported overall satisfaction with the YRE calendar in which they were working. The beneficial timing of the breaks and intersession was reported by $157(30.8 \%)$ participants. $146(28.7 \%)$ of the participants on the qualitative question reported that the YRE calendar provided them personal and professional advantages, benefits, and opportunities. Many participants (124, 24.4\%) reported the YRE calendar breaks "rejuvenated," "renewed," "refreshed," "re-energized," and "recharged" them in their personal and professional lives. See Table 10 for further response breakdowns and Appendix $\mathrm{Z}$ for respondent comments. 


\section{Table 10}

Frequencies and Percentages of Participants' Responses Regarding Teacher and Student Benefits of the YRE Calendar - Positive and Negative

\begin{tabular}{lccccc}
\hline $\begin{array}{l}\text { Comment } \\
\text { Breakdowns }\end{array}$ & $\begin{array}{c}\text { Total } \\
\text { Comments }\end{array}$ & $\begin{array}{c}\text { Frequencies } \\
\text { of Positive } \\
\text { Comments }\end{array}$ & $\begin{array}{c}\% \text { of } \\
\text { Positive } \\
\text { Comments }\end{array}$ & $\begin{array}{c}\text { Frequencies } \\
\text { of Negative } \\
\text { Comments }\end{array}$ & $\begin{array}{c}\% \text { of } \\
\text { Negative } \\
\text { Comments }\end{array}$ \\
\hline $\begin{array}{l}\text { Benefits for } \\
\text { Teachers }\end{array}$ & 503 & 454 & $90.3 \%$ & 49 & $9.7 \%$ \\
$\begin{array}{l}\text { Benefits for } \\
\text { Students }\end{array}$ & 332 & 312 & $93.9 \%$ & 20 & $6.1 \%$ \\
Totals & 835 & 776 & $91.7 \%$ & 69 & $8.3 \%$
\end{tabular}

Note: $N=562$ Respondent making comments

509 of 562 Respondents made Positive Comments on YRE Impacts

53 of the Respondents made Negative Comments on YRE Impacts

Respondents' multiple comments were broken down across two categories:

Benefits of Teachers and Benefits for Students - totaling 835 comments

Teacher responses consistently communicated two categories: Benefits for

Teachers and Benefits for students. Benefits for Teachers included teacher beliefs in what the YRE calendar does for them personally and professionally. Benefits for Students included teacher beliefs in what the YRE calendar does for students directly through frequent vacations/breaks and indirectly through added-value instruction and learning provided through the Benefits to Teachers. Perceived teacher benefits were described as facets of the YRE calendar that teachers enjoyed, loved, appreciated, liked or with which they were pleased. Within the participant responses were statements conveying, "I believe" and "Ifeel." Perceived student benefits were described as student characteristics (e.g. rested, relaxed, re-charged, motivated) resulting from frequent vacations/breaks and increased instructional and learning opportunities resulting from the effect of the YRE frequent vacations/breaks on teachers (e.g. intersessions, better planned lessons, creative 
activities). Table 10 indicates that respondents made a total of 503 comments on YRE calendar teacher benefits. A total of $454(90.3 \%)$ of the comments on teacher benefits were positive. A total of $49(9.7 \%)$ of the comments on teacher benefits were negative. Respondent comments regarding teacher benefits support this research on school climate, school culture, teacher efficacy, collective efficacy, teacher job satisfaction, and teacher intent to turnover in the context of the YRE school calendar. Positive comments regarding professional and personal teacher benefits are as follows:

- (YRE) made my performance as an educator better. I especially like the 2 week breaks that are an essential component of the alternative calendar in my school district. I am convinced that I am healthier (mentally and physically) due to the alternative calendar

- I feel it (YRE) has aided in my grow(th) as a teacher and enabled me to make success a possibility for my students.

- (YRE) helped me to plan my year more effectively.

- (YRE) enabled me to teach in a school with a staff that for the most part are all positive. Both the teachers and the students do not seem to get "burned out" as fast due to the frequent breaks.

- (YRE) allowed me to teach more years. No long periods of time in school without a break.

- The year-round calendar is wonderful! I would NOT under any circumstances want to return to a traditional calendar!

- I would not work in a district that did not offer the year-round calendar. I can not imagine not having the breaks to plan. The students come back refreshed and ready to work. I recommend the year-round calendar.

- It has given me the opportunity to take a much needed break from the hectic schedule of teaching second grade. I return after the break refreshed and excited about teaching. It will help me stay in the classroom. It gives me the time to reflect on what I have taught.

- I have only taught in a year-round calendar, but when I think of how exhausted I am by break and how refreshed I feel after break; I can't imagine teaching in a traditional calendar. 
- Given me greater flexibility. It reduces teacher 'burn-out' and fosters positive attitudes among teachers. Spirits are more even throughout the year and teachers' tempers are better, attitudes more positive. I would not want to consider teaching in the traditional calendar.

- (YRE) boosted morale. I love it and would not work at a school without it.

- (YRE) has been wonderful. There are so many advantages to this calendar, both professionally and personally. I would not be happy to go back to a traditional calendar.

- I don't know how we made it with the traditional calendar. Teachers need breaks ..., so why not spread out the school year to include breaks? Planning is easier.

- I love the 2 week break. It seems to hit just when I'm running out of energy.

- This calendar keeps me from reaching burn-out, just when I am getting to the point where energy is beginning to flag.

- (YRE) has been a great opportunity. I would definitely be upset if we thought about changing it. I think teachers need the break in the Fall. It is overall a win win situation for everyone involved. I have nothing negative to say about the calendar

- The YRE calendar allows me time to rest and recoup as a teacher and mom, but I still have time during the breaks to come into my classroom and make some changes that benefit the students learning

- I think the year-round calendar is great. It helps break up the school year a little bit better, and now that I'm used to it, I don't think I'd want to work on a traditional calendar.

- Without the breaks, I think my satisfaction would be much less and would have to look for a new position.

- Personally (YRE) kept me from having to take "mental health days" around October, November, and February, due to the wonderful fall, winter, and spring breaks.

- (YRE) provided valuable opportunity to network with staff in order to improve upon my professional knowledge as well as create plans of action in the ongoing school year instead of just in the summer breaks. The year-round calendar provides a much needed respite.

- (YRE) has given me more flexibility to manage my personal and professional life. 
- (YRE) enabled me to more effectively plan and provide instruction that has a focus and motivates my students.

- The Year-round calendar makes teachers more motivated to teach because they are getting lots of little breaks to rejuvenate themselves.

Negative comments regarding professional and personal teacher benefits were as follows:

- My negative feeling toward year-round education stem from the hardships that I think it brings on students and teachers that are involved in extra-curricular activities that force them to lose portions of their break.

- I would rather have a traditional calendar and have a longer summer break hecause I usually take classes or staff development over the summer and then there is no time left. Also, the two week breaks are a little to long, especially during the time of year.

- I have not had the opportunity to get employment in the traditional summer vacation time. This limits my ability to create income when I am unemployed. Teaching is a wonderful job and I would hate to have to leave it and go to the private sector.

- (YRE) caused me to have to work during the time we have off. Some of our outside events take place at a time that causes me to have to prepare students while they're on the break.

- It can be difficult to take college classes in the summer, because of the schedule.

- The only major concern I have with the year-round education calendar is CATS testing in the Spring. We (year-round districts) have the same testing window as those who follow the traditional school calendar.

- (YRE) created conflict with my family. I teach at a different district than my children. The school calendars sometimes do not match, and planning a vacation can be difficult.

Teachers' negative comments regarding teacher benefits of the YRE school calendar cluster concerned extra-curricular activities, traditional summer professional development and pursuing advanced degrees from Universities operating on a different calendar, 
summer employment opportunities and achievement press of state and national accountability assessment.

Overall, these responses concerning teacher benefits are consistent with previous research on the effect of YRE school calendars by Adams, (2001), Donohue, (1997), and Boyles (1993). Teachers made references to a number of the variables included in the quantitative part of the study: school climate and school culture (environment), teacher effectiveness (teacher efficacy and collective efficacy), job satisfaction (teacher global and YRE job satisfaction) and teachers' intent to turnover if schools go away from the YRE calendar. These rich, qualitative comments corroborated many of the findings the quantitative study. From comment analyses, teachers in this study were more likely to value working in a school and a school calendar that fosters high student engagement, effective instructional practices and sound classroom management. Based on the summary of comments on teacher benefits, teachers in this study demonstrated strong satisfaction with school climate, school culture, teacher efficacy, and collective efficacy. Given the frequency and percentage of positive comments regarding teacher benefits of the YRE calendar, teachers in this study were less likely to experience intent to turnover.

Table 11 indicates that respondents made a total of 332 comments on YRE calendar student benefits. A total of $312(93.9 \%)$ of the comments on student benefits were positive. A total of $20(6.1 \%)$ of the comments on student benefits were negative. Respondent comments regarding teacher perceptions of benefits for students support this research on school climate, school culture, teacher efficacy, collective efficacy, teacher job satisfaction, and teacher intent to turnover in the context of the YRE school calendar. Respondent comments regarding student benefits are as follows: 
- Children remain in a constant positive environment and the calendar also helps us to not have to set up a new school routine every year.

- I believe that the learning environment, including teacher morale, makes the greatest impact on student achievement.

- (YRE) provides a good learning environment for special education students.

- (YRE) provides a better teaching environment. I see a positive affect on the student and teacher morale with the extended breaks throughout the year.

- Students who need additional instruction are given more in depth assistance in small groups.

- The burn-out-rate for students is not as high as it would be if we were following a traditional calendar year.

- The Year-round calendar allows students to "rest and refresh" between chunks of intense learning. I feel that helps them learn to the best of their personal abilities over-all!

- The year-round calendar's breaks lessen my job stress, give me opportunities to plan more interesting and effective units of study, and refresh my outlook with challenging students.

- The calendar is great; provides small periodic breaks throughout the year and students get a better education.

- (YRE) provided me with the opportunity to relax, refresh, and develop new, creative ideas and strategies to meet the needs of my students.

- The break gives me an opportunity to plan fun, innovative lessons for my students.

Negative comments regarding teacher perceive negative student benefits are as

follows:

- The only drawback is when you have young children (preschool) it is sometimes hard for them to adjust after being off for a while.

- My negative feeling toward year-round education stem from the hardships that I think it brings on students that are involved in extra-curricular activities that force them to lose portions of their break. 
- I do often notice a decrease in skills and performance ability among students (in practice, application, and assessment) upon returning.

- When there are extended periods of time without school throughout the school year, I have to re-teach so many things to get everyone back to the level I expect of them.

- In fact, the periodic breaks we get with YRE don't supplement retention at all. I find myself retracing my steps with classes to ensure they don't forget.

Teacher negative comments on student benefits from the YRE school calendar cluster around young students (e.g. preschool and kindergarten), extra-curricular activities, student retention, and teacher re-teaching previously learned concepts.

Overall, respondents indicate that the YRE school calendar yields enhancement to their school environment, instructional practices and the teaching learning community. These comments add support to the quantitative research on school climate and school culture. These rich, qualitative comments provide added value to the quantitative study. From comment analyses, teachers in this study were more likely to value working in a school and a school calendar that fosters high student engagement, effective instructional practices and sound classroom management. Based on the summary of comments on student benefits, teachers in this study demonstrate strong satisfaction with school climate and school culture for students affected by the YRE school calendar and perceived enhanced teacher efficacy and collective efficacy resulting from the personal and profession impacts of the YRE school calendar on teachers. Given the frequency and percentage of positive comments regarding student benefits of the YRE calendar, teachers in this study are likely to experience increased teacher efficacy, collective efficacy and job satisfaction. Respondents' comments indicate overall positive global job satisfaction and especially teacher YRE job satisfaction. Respondents expressed satisfaction with 
employment as a teacher, working in their schools, the school climate, their treatment by colleagues and schools, and finally, their opportunities to develop skills and abilities for the job. Teachers in this study were less likely probably to experience intent to turnover.

Although not variables under study in this research, teachers provided an extensive list of possible future study variables within the context of the YRE school calendar. Some of these new variables we found are as follows: teacher and student burnout, teaching and learning pace, teacher and student attendance patterns, school calendar flexibility, teacher resilience, creativity, reflection, and rejuvenation. These responses concerning teacher and student benefits are consistent with Adams (2001), Donohue, (1997), and Boyles (1993). These respondent comments supplement the quantitative research data on the variables of student engagement, inquiry practices, instructional practices and school climate.

\section{Summary}

The purpose of this chapter was to present the results of the statistical tests for this study in the form of descriptive statistics, reliability coefficients and statistics to answer research questions 1,2, and 3. According to the theoretical literature, school climate collegial leadership, school climate - institutional vulnerability, teacher efficacy instructional practices, collective efficacy and teacher YRE job satisfaction exert significant influence on teacher intent to turnover. The results of this research suggested a strong, positive relationship between teacher intent to turnover and specific variables of school climate, school culture, teacher efficacy, collective efficacy and teacher job satisfaction. The hierarchical regression analysis demonstrated that participants perceived that collegial leadership, inquiry practice, teaching learning community, task analysis 
collective efficacy, and teacher job satisfaction were indicators of teacher decisions to remain in their current employment position.

In addition, the analyses of the qualitative data suggest that respondents perceived working within a YRE school calendar to provide benefits and advantages to both teachers and students. The relevance of these findings further indicates that respondent perceptions of school climate, school culture, teacher efficacy, collective efficacy and teacher job satisfaction are effective indicators of their overall intent to turnover in working in a YRE school calendar.

Chapter V will present the implications of this study, recommendations for future research, and make conclusions. 


\section{CHAPTER V}

\section{DISCUSSION, IMPLICATIONS, RECOMMENDATIONS AND SUMMARY}

\section{Introduction}

Chapter V discusses the results of the current study obtained through the quantitative analyses of the internet-based questionnaire items and the qualitative data resulting from an open-ended question. The chapter is structured to interpret the findings by analyzing and drawing conclusions on the three research questions that guided this study. The primary purpose of this study was to identify the unique relationship of school climate, school culture, teacher efficacy, collective efficacy, and teacher job satisfaction and teacher intent to turnover. The three research questions that guided this study were:

1. To what extent do school climate, school culture, teacher efficacy, collective efficacy, teacher job satisfaction relate to teacher intent to turnover in the YRE context?

2. To what extent does teachers' length of employment in a YRE calendar relate to teacher intent to turnover?

3. After controlling for the background variables (i.e., gender, age, years of YRE teaching experience), what is the unique contribution of school climate, school culture, teacher efficacy, collective efficacy, teacher job satisfaction and intent to turnover 


\section{Summary of Results}

The first research question investigated the relationship school climate, school culture, teacher efficacy, collective efficacy, teacher job satisfaction relate to teacher intent to turnover in the YRE context. The second research question investigated the extent to which teachers' length of employment in a YRE calendar relates to teacher intent to turnover. The third research question, after controlling for the background variables, investigated the unique contribution of school climate, school culture, teacher efficacy, collective efficacy, teacher job satisfaction made on teacher intent to turnover. The findings have several implications for different stakeholders such as school boards, school administrators, teachers, students and community. An internet-based questionnaire was administered to a convenience sample of teachers who worked in school districts that had implemented a YRE calendar in the state of Kentucky $(N=748)$. This study also sought to determine which blocks of variables (i.e., demographic) predicted teacher intent to turnover with statistical significance.

The results of the analyses of quantitative and qualitative data presented in the previous chapter illustrated the unique relationship of school climate, school culture, teacher efficacy, collective efficacy, and teacher job satisfaction to the dependent variable of teacher intent to turnover. The results of this research suggested a moderate, positive relationship between teacher intent to turnover and specific variables of school climate, school culture, teacher efficacy, collective efficacy and teacher job satisfaction. The hierarchical regression analysis demonstrated that respondents perceived that collegial leadership and institutional vulnerability (school climate), instructional practices (school culture), collective efficacy and teacher YRE job satisfaction were predictors on teachers' 
decisions to remain in their current employment position, seek a similar job in another school district on a traditional school calendar or leave the teaching professional altogether. Recommendations and implications for implementation and future research concerning teacher intent to turnover in the context of YRE calendars were made.

The following sections are summarized below: statement of the problem, review of the method, summary of the results, and discussion of the results according to the three research questions, limitations, significance of the study, suggestions for additional research and summary.

\section{Statement of the Problem}

Although there is considerable empirical research on school climate (Hoy \& Sabo, 1998); Hoy et al., 2002), school culture (Kardos \& Others, 2001; Reames \& Spencer, 1998; Vail, 2005), teacher efficacy (Gresham, 2002; Hoy \& Woolfolk, 1990; Moore \& Esselman, 1994), collective efficacy (Joffres \& Haughney, 2001; Mawhinney et al., 2005; Parker, 1994), and teacher job satisfaction (Kim \& Loadman, 1994; Smith \& Ingersoll, 2004; Smith \& Rowley, 2005), little is known about teachers' intent to turnover in the context of YRE education. The literature on YRE reports positive effects of the calendar reform on teacher stress and burnout, and other common workplace descriptors (Adams 2001). Although the implementation of YRE to improve teaching and learning in school districts continues, empirical research to guide and understand YRE is lagging. A conceptual model, Conceptual Framework Model for Teacher Intent to Turnover in a YRE Context, was designed and conducted using a hierarchical regression analysis to examine the unique contribution of school climate, school culture, teacher efficacy, 
collective efficacy and teacher job satisfaction on teacher intent to turnover in YRE schools.

\section{Review of the Method}

This study used a target population that included classroom teachers working in year-round education calendar schools in nine school districts in Kentucky. Participants were sent an invitation to participate via e-mail and asked to complete an internet-based questionnaire with an embedded web-address. Respondents' data automatically downloaded to a server database and subsequently analyzed. The independent variables were demographic variables (ethnicity, age, gender, years teaching experience in YRE) school climate, school culture, teacher efficacy, collective efficacy, and teacher job satisfaction. The dependent variable was teacher intent to turnover. The two main statistical procedures were Pearson's $r$ correlations and hierarchical regression.

\section{Summary of the Results}

The internet-based study questionnaire was delivered to 1,254 respondents. After following multiple e-mail contacts (Schaefer \& Dillman, 1998), the study resulted in 748 useable surveys, with a response rate of $59.6 \%$. Out of 748 useable surveys, 345 questionnaires were returned after the first invitation to participate e-mail; 210 were returned after the second e-mail invitation; 188 were returned after a third e-mail invitation. The second and third e-mail invitation yielded $55.6 \%$ of the total surveys for the study. 


\section{Respondent Demographics}

Of the 748 respondents, $79 \%$ were female. Out of the 748 respondents, $4 \%$ were AfricanAmerican and 95\% were white/Caucasian. Respondents' age varied with (20\%) 21-29, (30\%) 30-39, (27\%) 40-49, (21\%) 50-49, and (2\%) 60 and greater.

\section{Measures Used}

School climate items on the questionnaire were the complete scale of the Organizational Climate Index (OCI), an organizational climate descriptive measure for schools developed by Hoy et al. (2002). School culture items on the questionnaire were the complete scale of the School Culture Indicators Scale, a measure of school culture indicators by McLaughlin \& Talbert (2003). Teacher efficacy items on the questionnaire were the complete scale of the Teachers' Sense of Teacher Efficacy Scale, developed by Tschannen-Moran \& Woolfolk Hoy (2001). Collective efficacy items on the questionnaire were the complete scale of the Collective Efficacy Scale developed by Goddard (2002a). Teacher job satisfaction items on the questionnaire were adapted from the Teacher Job Satisfaction Questionnaire (Lester, 1987). Following Boyles (1993), the final four questions of the Job Satisfaction Measure asked participants to rate their agreement on satisfaction with teaching in the YRE calendar. Teacher intent to turnover items on the questionnaire were adapted from the Michigan Organizational Assessment Questionnaire (MOAQ; Cammann et al., 1983), a measure of intent to turnover from current jobs or schools. Reliability coefficients on the scales were calculated, ranging from .67 to .93 for the scales measuring school climate, school culture, teacher efficacy, collective efficacy, teacher job satisfaction, and teacher intent to turnover. These reliabilities are consistent with previous on school climate (Hoy et al., 2002), school 
culture (McLaughlin \& Talbert, 2003), teacher efficacy (Tschannen-Moran \& Woolfolk Hoy, 2001), collective efficacy (Goddard, 2002a), teacher job satisfaction (Boyles, 1993; Lester, 1987), and teacher intent to turnover (Cammann et al., 1983).

\section{Discussion of the results according to the three research questions}

Based on the results of this study, this chapter will discuss the findings in relation to the literature and the relationship between school climate, school culture, teacher efficacy, collective efficacy, teacher job satisfaction and teacher intent to turnover in the YRE context, the relationship of the length of teachers' employment in a YRE calendar and teacher intent to turnover, and the unique contribution of school climate, school culture, teacher efficacy, collective efficacy, teacher job satisfaction to teacher intent to turnover, limitations of the study, and recommendations for future research. Relationship between School Climate, School Culture. Teacher Efficacy. Collective Efficacy, Teacher Job Satisfaction and Teacher Intent to Turnover in a YRE Context.

\section{School Climate}

To answer the first research question, Pearson product-moment correlation coefficients (Pearson's $r$ ) were used to examine the correlations between study variables (Vogt, 1999). The correlations were statistically significant at the .01 and .05 levels (2tailed). School climate total correlations with other study variables are consistent with previous research on school climate and teacher intent to turnover (Turan, 1998). Lower rating scores on school climate scale items indicated respondents' overall positive view of the climate of their schools. Higher school climate rating scores indicated a lesser positive or negative view of school climate. During correlational analyses, the variable 
school climate total yielded statistically significant positive correlation with intent to turnover $(.28 ; p<.01)$.

Results from the correlational analysis of school climate and intent to turnover indicated that teachers in these select YRE schools are less to turnover if the school climate is positive. Teachers in these schools are less likely to experience intent to turnover.

\section{School Culture}

School culture correlational results are consistent with previous research on school culture and teacher intent to turnover (Reames \& Spencer, 1998). Low scores on school culture scale items indicated respondents' overall positive view of the culture of their schools. Higher rating scores indicated a lesser positive or negative view of school culture. During correlational analysis, school culture total yielded strong statistically significant positive correlation with teacher intent to turnover $(.31 ; p<.01)$. Results from the correlational analysis of school culture and intent to turnover indicate a similar relationship with intent to turnover and school climate. Teachers in this study reported positive a school culture and were less likely to intend to turnover.

\section{Teacher Efficacy}

The teacher efficacy correlational results are consistent with previous research on the relation of teacher efficacy and teacher intent to turnover (Dee, 2002; Hoy \& Woolfolk, 1990). Low scores on teacher efficacy scale items indicated respondents' overall positive view of their teacher efficacy on how much they can do regarding student engagement, instructional practices, and classroom management. Higher rating scores indicated a lesser positive or negative view of their teacher efficacy. During correlational 
analysis, teacher efficacy total yielded strong to moderate statistically significant positive correlation with teacher intent to turnover $(.23 ; p<.01)$. Moderate correlation with teacher intent to turnover demonstrates that teachers in this study are less likely to experience teacher intent to turnover.

\section{Collective Efficacy}

Collective efficacy correlational results are consistent with previous research on the relation of collective efficacy and teacher intent to turnover (Mawhinney et al., 2005). Low scores on collective efficacy scale items indicated respondents' overall positive view of their collective efficacy in their school regarding group competence and task analysis. Higher rating scores indicated a lesser positive or negative view of their collective efficacy. During correlational analysis, collective efficacy total yielded strong to moderate statistically significant positive correlation with teacher intent to turnover $(.29 ; p<.01)$.

Results from the correlational analysis of collective efficacy and intent to turnover indicate teachers in this study are more likely to have more confidence in group competence and their abilities in task analysis and are less likely to intend to turnover.

\section{Teacher Job Satisfaction}

The teacher job satisfaction correlational results are consistent with previous research on the relation of teacher job satisfaction and teacher intent to turnover (Smith \& Ingersoll, 2004). Low scores on teacher job satisfaction scale items indicated respondents' overall positive view of their teacher job satisfaction in their job. Higher rating scores indicated a lesser positive or negative view of their teacher job satisfaction. During correlational analysis, teacher job satisfaction total yielded strong statistically 
significant positive correlation with intent to turnover $(.54 ; p<.01)$. Results from the correlational analysis of teacher job satisfaction and intent to turnover indicate that teachers in this study are less likely to experience intent to turnover.

\section{Teacher Intent to Turnover}

The teacher intent to turnover correlational results are consistent with previous research on the relation of teacher intent to turnover and school climate (Turan, 1998); teacher intent to turnover and school culture (Reames \& Spencer, 1998); teacher intent to turnover and teacher efficacy (Dee, 2002; Hoy \& Woolfolk, 1990); teacher intent to turnover and collective efficacy (Mawhinney et al., 2005); and finally, teacher job satisfaction and teacher intent to turnover (Smith \& Ingersoll, 2004). Low scores on teacher intent to turnover scale items indicated respondents' overall positive view of their overall intent to remain in their current job and school. Higher rating scores indicated an overall lesser intent to remain in their current job and school. During correlational analysis, teacher intent to turnover yielded strong to moderate statistically significant positive correlations with teacher job satisfaction total $(.55 ; p<.01)$ school culture total $(.31 ; p<.01)$, collective efficacy total $(.29 ; p<.01)$, school climate total $(.28 ; p<.01)$, and teacher efficacy total $(.23 ; p<.01)$. The positive correlations with these variables indicated that teachers in this study are less likely to intend to turnover.

Relationship of the Length of Teachers' Employment in a YRE Calendar and Teacher

\section{Intent to Turnover.}

Research Question \#2 explored to what extent does teachers' length of employment in a YRE calendar relates to teacher intent to turnover. To answer the research question, Pearson's $r$ correlations were used to investigate the strength and 
direction of relationships between teachers' length of employment in a YRE calendar and teacher intent to turnover. However, there was no significant correlation between years of YRE experience with teacher intent to turnover. This result does not support the literature on length of employment in the YRE school calendar and intent to turnover in Hasser \& Naser (2003) and Minnesota Department of Children, Families \& Learning (1999). Unique Contribution of School Climate, School Culture, Teacher Efficacy, Collective Efficacy. Teacher Job Satisfaction to Teacher Intent to Turnover

Based on theory and empirical research, hierarchical regression was used to test the Conceptual Framework Model for Teacher Intent to Turnover in a YRE calendar context. This statistical procedure was employed to determine how much variance in the dependent variable, intent to turnover, can be explained by a set of independent variables (Sweet \& Grace-Martin, 2003). The researcher determined the order of entry of the variables (i.e., blocks) into the regression equation, guided by theory and research. The quantitative results indicated that with gender, age, and years experience in YRE in the first block of the regression equation, $1.0 \%(p<.05)$ of the variance was explained in the regression equation (small effect size; Cohen, 1988). Only years of YRE teaching experience contributed unique variance to teacher intent to turnover.

After statistically controlling for demographic variables, the addition of school climate subscale scores (collegial leadership, professional teacher behavior, achievement press, institutional vulnerability), school culture subscale scores (inquiry practice, teaching learning community and collective problem solving) into the second block of the regression equation resulted in an additional 14\% $(p<.001)$ of the variance being predicted in the regression equation; a large size effect (Cohen, 1988). Of the separate 
variables, collegial leadership (school climate), inquiry practice, and collective problem solving (school culture) positively contributed to the intent to turnover regression equation (less likely to turnover).

After statistically controlling for the demographic variables, school climate subscale scores (collegial leadership, professional teacher behavior, achievement press, institutional vulnerability), school culture subscales scores (inquiry practice, teaching learning community and collective problem solving), the addition teacher efficacy subscale scores (student engagement, teacher instructional practices and classroom management) into the third block of the regression equation resulted in an additional $2 \%$ $(p=.01)$ of the variance being predicted in the regression equation; a large size effect (Cohen, 1988).

After statistically controlling for the prior three blocks of variables, the addition of collective efficacy subscale scores (group competence and task analysis) into the fourth block of the regression equation resulted in an additional $1 \%(p=.05)$ of the variance being predicted in the regression equation; a large size effect (Cohen, 1988).

Finally, after statistically controlling for the prior four blocks of variables, the addition of teacher job satisfaction subscale score (teacher job satisfaction global) into the fifth block of the regression equation resulted in 19\% variance being predicted in the regression equation; a large size effect (Cohen, 1988). Overall, the regression model predicted $38.0 \%$ of the variance in teacher intent to turnover. However, it should be noted that these results were obtained for one particular order of entry of the independent variables, based on the conceptual model in Figure 6 . 


\section{Conceptual Model}

\section{Conceptual Model}

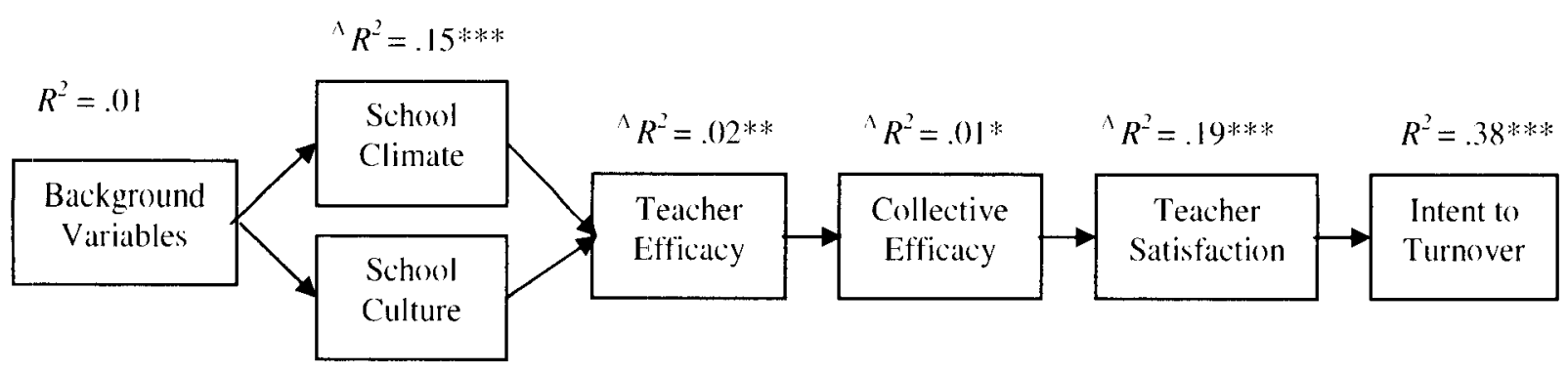

Figure 6. Conceptual Framework Model for Teacher Intent to Turnover in a YRE Context.

The beta coefficients at each step gave the relative importance of the predictor variables within that block. The largest beta coefficient in Step 1 was teachers' years of YRE teaching experience $(\beta=-.15)$. This demographic variable makes the strongest unique contribution to explain the dependent variable, when the variance explained by all the other variables in Step 1, are controlled. Analyzing Step 2, results indicate that teaching learning community (school culture subscale; $\beta=-.14$ ) makes the strongest unique contribution to explain the dependent variable followed by collegial leadership (school climate subscale) and inquiry practice (school culture subscale). Student engagement (teacher efficacy; $\beta=.14$ ) made the strongest unique contribution in Step 3 followed by instructional practices (collective efficacy). Step 4 results show that task analysis (collective efficacy; $\beta=.06$ ) makes the strongest predictors in Step 4 . The strongest unique contribution to explain the dependent variable intent to turnover was teacher job satisfaction global (teacher job satisfaction subscale; $\beta=.65$ ).

Hierarchical regression results support previous YRE research on school climate and school culture (Adams, 2001; Boyles, 1993; Donahue, 1997; Kennedy \& Wilcher, 
1998), teacher efficacy (Curry et al., 1997; Pittman \& Herzog, 1998; Shields \& Oberg, 2000; Worthen \& Zsiray, 1993), collective efficacy (Prohm and Baenen, 1996), teacher job satisfaction (Ballinger, 1999; Boyles, 1993; Donahue, 1997; McFadden, 2004; Sanders, 2001) and teacher intent to turnover (Kenyeri, 2002); Lui \& Li, 2005); VanderHooven, 1994). Respondents in this study had positive school climate, school culture, teacher efficacy, collective efficacy scores and are less likely to intend to turnover.

\section{Qualitative Data}

The qualitative part of the internet-based questionnaire was one open-ended question that asked participants: "For me, personally and professionally, the year round education calendar has ......" Results indicated that 562 of 748 respondents for this study chose to respond to the open-ended statement (75\%; See APPENDIX Z). The results from the open-ended question were examined for positive and negative responses to the open-ended question to provide further depth and breadth to the quantitative research findings. Comments that used positive descriptors (e.g. "like," "wonderful," "enjoy") were coded as a reported positive advantage for teachers or students. Comments that used negative descriptors (e.g. "do not like," "hate," "disadvantage") were coded as negative and as disadvantages for teachers and students. 509 of the $562(90.6 \%)$ responded favorably toward YRE while $53(9.4 \%)$ responded negatively toward YRE in their comments. Analyses indicated that teacher responses consistently communicated two categories: Benefits for Teachers and Benefits for students. The length, specificity, and details found in the responses indicated that there may be some differences in the level of advocacy for the YRE calendar across schools, districts, and teacher 
demographics, at the very least a difference in the enthusiasm level of teachers concerning the YRE calendar (See Table 12).

Respondents made a total of 503 comments on YRE calendar teacher benefits. A total of $454(90.3 \%)$ of the comments on teacher benefits were positive. These rich, qualitative comments corroborated many of the findings the quantitative study. From comment analyses, teachers in this study were more likely to value working in a school and a school calendar that fosters high student engagement, effective instructional practices and sound classroom management. A total of $49(9.7 \%)$ of the comments on teacher benefits were negative clustering around concern for extra-curricular activities, traditional summer professional development and pursuing advanced degrees from Universities operating on a different calendar, summer employment opportunities and achievement press of state and national accountability assessment. Respondent comments regarding teacher benefits support this research on school climate, school culture, teacher efficacy, collective efficacy, teacher job satisfaction, and teacher intent to turnover in the context of the YRE school calendar. Given the frequency and percentage of positive comments regarding teacher benefits of the YRE calendar, teachers in this study were less likely to intend to turnover.

Respondents made a total of 332 comments on YRE calendar student benefits. A total of $312(93.9 \%)$ of the comments on student benefits were positive. Respondent comments regarding teacher perceptions of benefits for students support this research on school climate, school culture, teacher efficacy, collective efficacy, teacher job satisfaction, and teacher intent to turnover in the context of the YRE school calendar. A total of $20(6.1 \%)$ of the comments on student benefits were negative clustering around 
young students (e.g. preschool and kindergarten), extra-curricular activities, student retention, and teacher re-teaching previously learned concepts.

Overall, respondents indicated that the YRE school calendar yields enhancement to their school environment, instructional practices and the teaching learning community. These comments add support to the quantitative research on school climate and school culture and added value to the quantitative study. Teachers demonstrated strong satisfaction with school climate and school culture for students affected by the YRE school calendar and perceived enhanced teacher efficacy and collective efficacy resulting from the personal and profession impacts of the YRE school calendar on teachers. The frequency and percentage of positive comments regarding student benefits of the YRE calendar indicates teachers in this study are likely to experience increased teacher efficacy, collective efficacy and job satisfaction. Respondents demonstrated overall positive global job satisfaction and were less likely to intent to turnover.

\section{Limitations}

Although the current study makes a significant to understanding the unique relationship of school climate, school culture, teacher efficacy, collective efficacy, teacher job satisfaction to teacher intent to turnover theories, there are a number of limitations that should be addressed.

First, the lack of randomization among schools, school districts and teachers would prevent larger generalizations of the results. As this was an exploratory study, generalization of the results beyond the present study are not valid because the participants were drawn from an "intact group," teachers from schools in nine school districts that were operating on YRE school calendars and were able to provide the 
requested data via an online questionnaire. An internet-based instrument was appropriate for this study because the participants were available online and had, through their participation in a YRE school calendar, demonstrated at least a basic level of understanding of the impacts of a YRE school calendar.

Previous studies on teacher intent to turnover have indicated a need for an investigation of a multiple variables that may influence teachers' decisions to transfer schools or leave the profession altogether (e.g., job satisfaction, workload, student behavior, lack of planning time, lack of influence on policy decisions, time with family; Alliance for Excellent Education., 2005). This study drew from research on school climate, school culture, teacher efficacy, collective efficacy, and teacher job satisfaction and what unique correlation exits between these variables and teacher intent to turnover. Due to the nature of influence of these variables on teacher intent to turnover, this study may be an important contribution to the empirical research in the field study of teacher attrition and turnover. However, the school calendar design, leadership, and instructional staff might have influenced the study results. As such, these results may not be representative of other school and school district settings operating on a YRE calendar schedule.

The selection and size of the population used for this study also may limit the generalization of the findings. Future studies are warranted to investigate the variables of this study with different populations - more diverse sample of teachers - and in different school calendar - YRE and traditional school calendars, to further validate the findings of this study. 
The response rate to an internet-based questionnaire is another limitation. Electronic response rates tend to be lower when compared to mailed surveys. Low response rates may result in a biased estimate of the characteristics of the population (Bean \& Roszkowski, 1995). This study achieved approximately a $60 \%$ response rate from the respondent pool. Although this response rate is consistent with previous research on internet surveys, (Crawford et al., 2001; Simsek \& Veiga, 2001), additional studies using this research methodology should be done to further test the conceptual model proposed in this study. The demographic profile of the participants in this study was congruent with previous studies about YRE school calendars as females comprised more than $80 \%$ of the sample and the majority were teachers between the ages of $30-49$.

Another limitation is that the results from this research are reliant on teachers' self-reports and perceptions to the items on the questionnaire. Perceptions can be left open to interpretation and can involve attitudes, feelings, and beliefs that are subject to change over time. However, this does not discount the benefit that self-report questionnaires can provide as it is evident from reviewing other studies that there is value in measuring perceptions (Picciano, 2002). Ultimately, it is teachers' perceptions of their working experience that may be the catalyst for a teacher to continue employment in a particular school or school district.

Another limitation of this study is that it takes into consideration only the perceptions of the teachers who responded to the questionnaire. The participants who chose to participate (748) may represent a distinct population with characteristics that differ from the population as a whole (1254). The viewpoints of the teachers who either did not respond (506) were not included in the data. Future studies should attempt to 
explore the viewpoints from those teachers who chose not to complete the questionnaire to more clearly understand what factors may have played a stronger role in their decision not to participate.

Another possible limitation of the study could be that the present study used only an internet-based questionnaire. The results obtained by the self-report scales may have been inflated by common method bias (Noe \& Wilk, 1993). The general demographic composition of the sample might also prevent generalizing the results, as more than $80 \%$ of the sample was female, and, $95 \%$ of the respondents were Caucasian.

\section{Significance of the Study}

The results of this study may be important to a number of educational stakeholders such as school boards, administrators, teachers, parents and local policy makers. Acknowledging and addressing intent to turnover is particularly important, given the costs associated with teacher turnover and the disproportionate impact teacher turnover has on low-income and minority students. Increasing teachers' intent to remain in the teaching profession is vital to local, state and national educational accountability.

The Conceptual Framework Model for Teacher Intent to Turnover in a YRE Context below demonstrated that $38 \%$ of the variance on intent to turnover can be accounted by the study variables. This amount of variance from the model magnifies the importance that schools should place on the teacher workplace.

Results of this study can provide useful for policymaker interested in implementing the YRE school calendar in schools to create the school climate and school culture described the teachers in this study. In the qualitative research in particular, teachers described a school climate that is characterized by positive collegial leadership, 
professional teacher behavior, achievement press and institutional vulnerability. They described school culture that emphasizes inquiry practices, a teaching and learning community and collective problem solving. Teachers reported positive student engagement, effective instructional practices and classroom management. Teachers described school staffs as having positive group competence and task analysis. Teachers indicated positive satisfaction with teaching and especially with their YRE school calendar. Most importantly, teachers reported very low interest in intent to turnover. This low level of intent to turnover is potentially_powerful for policymakers, teachers and communities that may be considering implementing a YRE school calendar. Teacher turnover is extremely costly not only in funds spent replacing the teacher who leaves but in the loss or drain on instructional expertise.

Teachers who work within an environment that fosters these characteristics are more likely to be successful with student and school accountability measures. Students are more likely to benefit from teachers' positive teacher efficacy and collective efficacy. Schools are less likely to experience the loss of instructional expertise as teachers in this study are less likely to experience intent to turnover. Teachers reported that, with the frequent vacations/breaks, students are more likely to experience teachers who are rested, relaxed, rejuvenated, and more effective instructional practices.

\section{Suggestions for Additional Research and Summary}

1. Because the results of this research should only be generalized to a similar population, more research is needed with teacher populations to validate the results of this study. Thus, a test of generalizability would be to determine if the independent study variables had similar influences on teacher intent to turnover. Further research is 
needed that helps develop a better understanding of conditions or factors that may be both related and not related to teacher intent to turnover. For example, $87 \%$ of the participants in this study reported they were, "All in all, satisfied with their experience teaching in a YRE calendar." and 64\% reported that it is "Not At All Likely," if they had the chance to take a similar job in a traditional school calendar, that they would do so. Research is needed to determine the extent that perception of intent to turnover in the context of YRE and traditional school calendars.

2. A study should compare the school climate of YRE calendar schools with school climate of traditional calendar schools. The number of YRE calendar schools is increasing. Public school leaders may want to consider a YRE school calendar that, according to teachers in this study, may create a positive, open, healthy school climate for teaching and learning for those who attend public schools.

3. A study could be designed to test the Conceptual Model in a different context that would have more balanced ethnicity populations, more male teachers and more balance among teacher school instruction levels.

4. A longitudinal cohort comparison of two groups of teachers over time - one group working in a YRE school calendar and one group working in a traditional school calendar.

5. Replication of this study in the context of urban school districts would provide intent to turnover data from school districts comprised of different variations in teacher demographics. Additional studies would provide comparative information as well as a larger area for which findings can be generalized. 
6. Replication of this study in the context of YRE schools that operate on a YRE calendar while other schools in the school district operate on a traditional school calendar would provide interesting data on study variables.

7. Replication of this study in the context of school districts that do not operate with school-based decision making councils could provide interesting data on study variables and school leadership.

8. Replication of this study in the context of specialized instructional staff (e.g. special education teachers, English as a second language teacher, limited English language teachers, and vocational education teachers) could provide interesting data on study variables.

9. A study could be designed to explore variables that teachers reported in the qualitative data analysis. The variables of study could be: teacher and student burnout, teaching and learning pace, teacher and student attendance patterns, school calendar flexibility, teacher resilience, creativity, reflection, and rejuvenation.

\section{Summary}

This study was designed to provide a better understanding of the relationship of the independent variables of school climate, school culture, teacher efficacy, collective efficacy, and teacher job satisfaction with the dependent variable of teacher intent to turnover in the context of a YRE school calendar. Three research questions were designed to guide the study. A quantitative internet-based questionnaire research design was selected and described in Chapter III. Guided by the literature, a Conceptual Framework Model for Teacher Intent to Turnover was designed to test the variance the independent variables contributed to intent to turnover in the context of the YRE school 
calendar. Chapter IV presented research results. This chapter presented a discussion of the results of the relationships between the variables explored in this study. The results suggested that, although there was strong positive correlation all independent study variables, teacher job satisfaction accounted for the greatest variance of teacher intent to turnover. Furthermore, the results demonstrated weak statistically correlation existed between intent to turnover and other study variables. A conceptual model related to this relationship was presented and discussed.

In summary, school calendar designers may want to address the requirements of effective educational environments that are of high quality, effective, and best meet the needs of the teachers and students. The ability to express and share ideas among learners and with the instructor is more likely to promote collaboration and deepens the learning experience. It is important to design school calendars that provide a satisfying and effective working environment. The results of this study suggested that positive levels of perceived collegial leadership, institutional vulnerability, instructional practices, collective efficacy and YRE job satisfaction, have a direct influence on both teacher job satisfaction and teacher intent to turnover. Furthermore, viable exploratory strategies were provided to address the variables in this study that could influence teacher intent to turnover.

By fostering a teacher working environment that will demonstrate a conducive school climate, school culture, collegial leadership, controlled sense of institutional pressure from outside, instructional practices, collective sense of efficacy and teacher YRE job satisfaction, teachers' levels of job satisfaction, perceived quality of the working environment, and ultimately, teachers' motivation to persist with the school will be 
improved. The facilitation of teacher motivation and commitment may positively influence learning outcomes by improving students' involvement in the learning process, thus, contributing to potentially more student interest in the content matter and enhancing collaboration during learning activities to construct knowledge and negotiate meaning through critical thinking.

It is hoped that the outcomes of this study, in examining the issues of teacher intent to turnover in the context of a YRE school calendar and its possible usefulness in teacher retention, may help to provide some valuable information and increase the understanding that teacher needs, experiences, and perspectives may influence an optimal learning environment for students. 


\section{REFERENCES}

Adams, R. L. (2001). Stakeholder Beliefs, Satisfaction, and Assessments of School Climate after Implementation of a Year-Round Calendar. Unpublished Dissertation Electronic Theses and Dissertation, East Tennessee State University.

Alavi, H. R. (2005). The organizational climate of Kerman Shahid Bahonar University: Its comparison with the desired organizational climate from the viewpoints of the personnel (staff) of the university. Public Personnel Management. Retrieved October 23, 2006, from http://www.findarticles.com/p/articles/mi_qa3779/is_200510/ai_n15704779

Alliance for Excellent Education. (2005). Teacher attrition: A costly loss to the nation and the states. Issue Brief. Alliance for Excellent Education.

Anderson, L. W. \& Walberg, H. J. (Eds.). (1993). Timepiece: Extending and enhancing learning time. San Diego, CA: National Association for Secondary Principals.

Anderson, L. W. (1990). Likert scales. In H. Walberg \& G. Haertel (Eds.). The International Encyclopedia of Educational Evaluation. Elmsford, NY: Pergamon Press.

Anderson, W. M. (2002). Factors that motivate teachers to remain in the teaching profession in North Carolina. Unpublished Dissertation. Dissertation Abstracts International Section A: Humanities and Social Sciences, 62(9-A), 2929.

Anoff, P. (1999). Growing the year-round education movement. Paper presented at the annual conference of the National Association for Year-Round Education, San Diego, CA.

Ashton, P. T. \& Webb, R. B. (1983). A study of teachers' sense of efficacy. (No. 400-790075, Final Report, Executive summary, National Institute of Education Contract). Gainesville, FL: University of Florida, (ERIC Document Reproduction Service No. ED 213834 ).

August, L. \& Waltman, J. (2004). Culture, climate, and contribution: career satisfaction among female faculty. Research in Higher Education 45(2), 177-192.

Baird, D. (2005). Year-round leader. District Administration 41(10), 24-24. 
Ballinger, C. E. (1999). A bridge to better learning: Annual report to the association on the status of year-round education. Paper presented at the annual conference of the National Association for Year-Round Education, San Diego, CA.

Ballinger, C. (1997). Unleashing the school calendar. Thrust for Educational Leadership, 16, 16-18.

Ballinger, C. E. (1989). The case for year-round education: An idea whose time has come. San Diego, CA: National Association for Year-Round Education.

Ballinger, C. E. (1988). Rethinking the school calendar. Educational Leadership, 45, 5761.

Ballinger, C. E., Kirschenbaum, N., \& Poinbeauf, R. P. (1987). The various year-round plans. The Year-Round School: Where Learning Never Stops. Bloomington, IND: Phi Delta Kappa, 16-24.

Bandura, A. (1977). Self-efficacy: Toward a unifying theory of behavioral change. Psychological Review, 84, 191-215.

Bandura, A. (1982). Self-efficacy mechanism in human agency. American Psychologist, $37,122-147$.

Bandura, A. (1993). Perceived self-efficacy in cognitive development and functioning. Educational Psychologist, 28, 117-148.

Bandura, A. (1996). Self-efficacy in changing societies. New York: Cambridge University Press.

Bandura, A. (1997). Self-efficacy: The exercise of control. New York: W. H. Freeman.

Bandura, A. (1998). Self-efficacy: The exercise of control New York: W.H. Freeman and Company.

Bandura, A. (2001). Guide for constructing self-efficacy scales (revised March 2001). Stanford University. http://www.emory.edu/EDUCATION/mfp/effguide.PDF

Barrett, M. J. (1990, September). The case for more school days. The Atlantic Monthly, 78-106.

Barron, R. 1993. The effects of year-round education on achievement, attendance and teacher attendance in bilingual school. Doctoral Dissertation, Northern Arizona University. Dissertation Abstracts International, 54, 3935.

Barth, R. S. (2006). Improving relationships within the schoolhouse. Educational Leadership 63(6), 29-33. 
Bean, A.G., \& Roszkowski, M.J. (1995). The long and short of it. Marketing Research, 7, 20-26.

Berliner, D. C. (2001). Improving the quality of the teaching force: A conversation with David C. Berliner. Educational Leadership, 58, 6-10.

Best, S. J., \& Krueger, B. (2002). New approaches to assessing opinion: The prospects for electronic mail surveys. International Journal of Public Opinion Research, 14, 73-92.

Boyles, B. W. (1993). Year-round education: Implementing the first two years in the elementary grades. Unpublished Doctoral Dissertation, University of North Carolina at Greensboro, Greensboro.

Bradford, J. C. (1993). Making year-round education (YRE) work in your district: A nationally recognized single track high school model. Paper presented at the Annual Meeting of the National Association for Year-Round Education, Anaheim, CA. Washington, D.C.: U.S. Department of Education, Office of Educational Research and Improvement (ERIC Document Reproduction Service No. Ed 358 559).

Bradford, J. (1992b). Year-round education: A national perspective. Paper presented at the National Commission on Time and Learning, Mufreesboro, TN.

Bradford, J. C. (1991). Year-round schools: A national perspective. Buena Vista, VA: Buena Vista School District. (ERIC Document Reproduction Services No. 343259).

Bradford, J. D. (1990). Year-round schooling: A school for all seasons at the secondary level. Buena Vista, VA: Buena Vista School District. (ERIC Document Reproduction Services No. 343 260).

Branham, F. L. (2006). Six truths about employee turnover. American Management Association.

(http://www.collegecertificates.com/books/catalog/0814405975_s.htm)

Brannick, J. (1999). Decreasing the staggering costs of turnover in your organization. Retrieved December12, 2005, from http://www.brannickhr.com/ArticleRetention.html.

Bray, S. \& Roellke, C. (1988). The evidence on year-round education. Kappa Delta Pi Record, 15, 84-88.

Brekke, N. (1992). "Year-round schools: An efficient and effective use of resources." School Business Affairs, pp. 27-37, cited in Kneese, C.C. 1996. "Review of Research on Student Learning in Year-Round Education." Journal of Research and Development in Education, 29, 60-72. 
Brekke, N. R. (1992c). You're really efficient and effective. Oxnard, CA: Oxnard School District.

Brekke, N. R. (1990). YRE: A break from traditional that makes educational and economic sense. Washington, D.C.: U.S. Department of Education, Office of Educational Research and Improvement (ERIC Document Reproduction Service No. Ed 324 818).

Brekke, N. R. (1984). Year-round education: Cost saving and educationally effective. ERS Spectrum, 2, 25-30.

Brenner, D. B. (2004). Teacher job satisfaction in a reform environment: The influence of job dimensions, psychological states, school characteristics, and teacher characteristics. Unpublished Dissertation, Dissertation Abstracts International Section A, 65(1-A), 27.

Brown, O. M. (2001). The relationship between organizational climate and job satisfaction of selected urban middle school teachers in the Clark County School District (Nevada). Dissertation Abstracts International Section A: Humanities and Social Sciences, 62(5-A), 1644.

Bulach, C. R. \& Berry, J. (2001). The Impact of Demographic Factors on School Culture and Climate. Paper presented at the Annual Meeting of the Southern Regional Council on Educational Administration (Jacksonville, FL, November 1-4, 2001).

Camman, C., Fichman, M., Jenkins, G., \& Klesh, J. (1983). Assessing attitudes and perceptions of organizational members. In S. Seashore, E. Lawler, P. Mirvis, \& C. Cammann (Eds.). Assessing organizational change: A guide to methods, measures, and practices (pp. 71-138). New York: John Wiley.

Caprara, G. V., Barbaranelli, C., Steca, P., \& Malone, P. S. (2006). Teachers' selfefficacy beliefs as determinants of job satisfaction and students' academic achievement: A study at school level, Journal of School Psychology, 44(6), 473490.

Carini, R. M., Hayek, J. C., Kuh, G. D. \& Ouimet, J. A. (2001). College Student Responses to Web and Paper Surveys: Does Mode Matter. at http://www.indiana.edu/ nsse/acrobat/mode.pdf, November 2002.

Cavanagh, S. (2005). Teacher turnover cost. Education Week, 25(2), 16-16.

Chambers, S. M. (2003). The impact of length of student teaching on the self-efficacy and classroom orientation of pre-service teachers. Paper presented at the annual meeting of the Southwest Educational Research Association, San Antonio, TX. (ERIC Document Reproduction Service No. ED 477 509). 
Cheng, Y. C. (1996). Relation between teachers' professionalism and job attitudes, educational outcomes, and organizational factors. Journal of Educational Research, 89, 163-171.

Chicago Acorn. (2003). "Where have all the teachers gone? The cost of teacher turnover in ACORN neighborhood schools in Chicago. Association of Community Organizations for Reform Now. Retrieved January 6, 2007 from http://www.acorn.org/index.php?id=8369

Christie, S. (1989). Report on opinion surveys of parents, students, and staff on four-track year-round schools in Cajon Valley, 1987-1988. Washington, D.C.: U.S. Department of Education, Office of Educational Research and Improvement (ERIC Document Reproduction Service No. Ed 303 888).

Colgan, C. (2004b, September). Losing teachers proves costly to schools. American School Board Journal, 9-10, 12.

Colgan, C. (2004a, August). Is there a teacher retention crisis? American School Board Journal, 191, 22-25.

Cook, C., Heath, F. \& Thompson, B. (2000, August). A new culture assessment: Preliminary report on the ARL SERVQUAL Survey. Proceedings of the IFLA Council and General Conference, Israel. Retrieved August 2, 2005, from the ERIC database.

Cookson, Jr., P. W. (2005, April). A community of teachers. Teacher PreK-8 35(7), 12 14. Retrieved on January 7 from http://www.teachingk8.com/archives/your_first_year/a_community_of_teachers_by_peter_w_cookson_ jr.html

Cooper, H., Valentine, J., Charlton, K., \& Melson, S. (2003). The Effects of Modified School Calendars on Student Achievement and on School and Community Attitudes." Review of Educational Research, 73, 1-52).

Cooper, H., Nye, B., Charlton, K., Lindsay, J. \& Greathouse, S. (1996). The effects of summer vacation on achievement test scores: A narrative and meta-analytic review. Review of Educational Research, 66, 227-268.

Costa, J. S. (1987). Comparative outcomes of the Clark County School District Yearround and nine-month schools (Nevada). (Unpublished Doctoral Dissertation. University of Nevada, Las Vegas). Dissertation Abstracts International 48/10A. 2495.

Couper, M. P. (2000). Web surveys: A review of issues and approaches. Public Opinion Quarterly, 64, 464-481. 
Couper, M. P. (2001). The promises and perils of Web surveys. In A. Westlake et al. (Eds.), The Challenge of the Internet, (pp.35-56). London: Association for Survey Computing.

Crawford, S.D., Couper, M.P., \& Lamias, M.J. (2001). Web surveys: Perceptions of burden. Social Science Computer Review, 19, 146-162.

Croasmun, J., Hampton, D. \& Hermann, S. (2006). Teacher Attrition: Is Time Running Out? Issues Challenging Education. Teacher Attrition Issue. The University of North Carolina at Chapel Hill. Retrieved from http://horizon.unc.edu/projects/issues/papers/Hampton.asp on December 18, 2006.

Cronbach, L. J. \& Shavelson, R. J. (2004). My current thoughts on coefficient alpha and successor procedures. Educational \& Psychological Measurement, 64, 391-418.

Crosby, E. A. (1999). Urban school: Forced to fail. Phi Delta Kappan, 81, 298-303.

Cross, C. T. \& Goldberg, M. (2005). Time is a Resource We Still Haven't Figured Out How to Use Wisely. Prisoners of Time. Education Commission of the States at www.ecs.org. Retrieved January 7, 2006 from http://www.edutopia.org/magazine/ed larticle.php?id=art_1333\&issue=sep_05

Curry, J. Washington, W. \& Zyskowski, G. (1997). Year-round schools evaluation, 199697. Austin Independent School District, Austin, TX: Department of Accountability, Student Services and Research. (ERIC Document Reproduction Service No. ED 414 326).

Curtis, K. C. (2005). An analysis of the construct of efficacy on new teacher retention. (Unpublished Doctoral Dissertation, George Mason University. UMI ProQuest Digital Dissertations.

Dale, R. L. (2005). The effects of teacher collaboration and school trust on collective efficacy. Unpublished doctoral dissertation, Oklahoma State University. Dissertation Abstracts International.

Darling Hammond, L. (2001). The challenge of staffing our schools. Educational Leadership, 58, 12-17.

Darling Hammond, L. (1996). The quiet revolution: Rethinking teacher development. Educational Leadership, 53, 4-10.

Daugherty, R., Kelley, R. C. \& Thornton, B. (2005). Relationships between measures of leadership and school climate. Education,126, 17-25. 
Dee, J. R. (2002). Turnover intent in an urban community college: Strategies for faculty retention. Paper presented at the annual meeting of the Association for the Study of Higher Education, Sacramento, CA. (ERIC Document Reproduction Service No. ED 471 151).

Dembo, M. H. \& Gibson, S. (1985). Teachers' sense of efficacy: An important factor in school improvement. Elementary School Journal, 86, 173-184.

Dejarnett, S. W. (1994). Year-round education: A synthesis of research. Unpublished Doctoral Dissertation, University of Georgia, Athens.

Deever, R. M. \& Shockley, H.A. (1975). Job satisfaction of teachers in selected extended school year program. Edacational Administration, 45, 57-61. (ERIC Document Reproduction Service No. ED 181583 ).

Dillman, D. A. (2000). Mail and Internet Surveys: The Tailored Design Methods. Second edition. New York: Wiley.

Dillman, D. A. \& Bowker, D. K. (2001). The Web Questionnaire Challenge to Survey Methodologists. Retrieved November 5, 2002 from http://survey.sesrc.wsu.edu/dillman/zuma paper dillman bowker.pdf.

Dillman, D. A., Tortora, R. D. \& Bowker, D. (1998). Principles for Constructing Web Surveys. Pullman, Washington. SESRC Technical Report 98-50. Retrieved November 5, 2006 from http://survey.sesrc.wsu.edu/dillman/papers/websurveyppr.pdf, November 2002.

Dillman, D. A., Tortora, R. D. \& Bowker, D. (1999). Principles for constructing web surveys. Social and Economic Research Center Technical Report 98-50, Pullman, WA.

Dillman, D. A., Phelps, G., Tortora, R. D., Swift, K., Kohrell, J. K. \& Berck, J. (2001). Response Rate and Measurement Differences in Mixed Mode Surveys Using Mail, Telephone, Interactive Voice Response and the Internet. Retrieved November 5 , 2002 from http://survey.sesrc.wsu.edu/dillman/papers/Mixed\%20Mode\%20ppr\%20_with\%2 OGallup \%20POQ.pdf.

Dillman, D. A., Tortora, R. D., Conradt, J. \& Bowker, D. (1998). Influence of Plain vs. Fancy Design on Response Rates for Web Surveys. Presented at Joint Statistical Meetings, Dallas, Texas. Retrieved November 5, 2002 from http://survey.sesrc.wsu.edu/dillman/papers/asa98ppr.pdf.

Donahue, S. L. (1997). Year-round education through the eyes of teachers. Unpublished Doctoral Dissertation, Florida State University, Tallahassee. 
Dore, T. L. (2005). The Relationships Between Job Characteristics, Job Satisfaction, and Turnover Intention Among Software Developers. Boca Roton, FL: Universal Publishers.

Dossett, D. \& Munoz, M. (2000). Year-round education in a reform environment: The impact on student achievement and cost-effectiveness analysis. Washington, D.C.: U.S. Department of Education, Office of Educational Research and Improvement. (ERIC Document Reproduction Service No. ED464424).

Dramstad, S. A. (2004). Job satisfaction and organizational commitment among teachers in Norway: A comparative study of selected school from public and private educational systems. Unpublished Dissertation. Dissertation Abstracts International Section A: Humanities and Social Sciences, 65(2-A), 409.

Dubay, T. L. (2001). Impact of a verbal persuasion treatment on teacher education students' attitudes and self-efficacy for computer technology. Dissertation Abstracts International Section A: Humanities and Social Sciences, 62(5-A), pp. 1799.

Duke, D. L. \& Gansneder, B. (1990). Teacher empowerment: The view from the classroom. Educational Policy, 4, 145-160.

Dunn-Wisner, K. A. (2004). The relationship among self-efficacy, perceived school climate, and stress in middle school teachers. Dissertation Abstracts International Section A: Humanities and Social Sciences, 65(4-A), 2004, 1247.

Elsberry, J. S. (1992). An evaluation of the implementation of year-round education. Unpublished Doctoral Dissertation, Dissertation Abstracts International, Vol 53(12-A), 4146-4147. The University of Texas at Austin, Austin.

Emerick, S., Hirsch, E. \& Berry, B. (2005, October). Teacher working conditions as catalysts for student learning. Association for Supervision and Curriculum Development: InfoBrief 43. Retrieved January 7, 2007 from http://www.ascd.org/portal/site/ascd/template.MAXIMIZE/menuitem.1f7f8e4alb 4bac58dd lb21 10d3108a0c/?javax.portlet.tpst=d5b9c0fa la493266805516f762108 a0c_ws_MX\&javax.portlet.prp_d5b9c0fa 1a493266805516f762108a0c_viewID=i ssue_view\&javax.portlet.prp_d5b9c0fa l a493266805516f762 108a0c_journalmoid $=\mathrm{f} 50885038 \mathrm{~b} 327010 \mathrm{VgnVCM} 1000003 \mathrm{~d} 01 \mathrm{a} 8 \mathrm{c} 0 \mathrm{RCRD} \&$ javax.portlet.begCacheT ok=token\&javax.portlet.endCacheTok=token.

Ettorre, B. (1997). Employee retention: Keeping the cream. HR Focus, 74, 1-3.

Falch, T. \& Strom, B. (2005). Teacher turnover and non-pecuniary factors. Economics of Education Review, 24, 611-631. 
Fardig, D. (1991). Year-round education program evaluation. Report of results. Washington, D.C.: U.S. Department of Education, Office of Educational Research and Improvement. (ERIC Document Reproduction Service No. ED 344 310).

Fitz-enz, J. (1997). It's costly to lose good employees. Workforce, 76, 50-51

Flowers, T. T. (2004). Why do public school teachers leave their professionals? Unpublished Dissertation, Dissertation Abstracts International Section A: Humanities and Social Sciences, 64(8-A), 2718.

Fowler, F. J. (1995). Improving survey questions: Design and evaluation. Thousand Oaks, CA: Sage Publications, Inc.

Fritts-Scott, K. A. (2005). The impact of year-round schooling on student achievement in Arkansas public elementary schools. Unpublished Dissertation, UMI ProQuest Digital Dissertation.

Gandara, P. \& Fish, J. (1994). Year-round schooling as an avenue to major structural reform, Educational Evaluation and Policy Analysis, 16, 67-85.

Gately, R. F. Cost of Employee Turnover [On-linel. Retrieved December 12, 2005, from http://ourworld.compuserve.com/homepages/gately/pp06trno.html

Gauci, B. R. \& Norman, I.J. (1997) Factors influencing turnover and absence of nurses: a research review. International Journal of Nursing Studies, 34, 385-394.

Gautham, T., Van Dick, R., and Wagner, U. (2004). Organizational identification and organizational commitment: Distinct aspects of two related concepts. Asian Journal of Social Psychology, 7, 301-315.

Gaziel, H. H. (2004). Predictors of absenteeism among primary school teachers. Social Psychology of Education, 7(4), 421-434.

Gibson, S. \& Dembo, M. (1984). Teacher efficacy: A construct validation. Journal of Educational Psychology, 76, 560-582.

Glines, D. (1995). Year-round education: History, philosophy, future. San Diego, CA: National Association for Year-Round Education.

Glines, D. (1994). Philosophical rationale for year-round education. Paper presented at the Annual Meeting of the National Association for Year-Round Education, San Diego, CA. Washington, D.C.: U.S. Department of Education, Office of Educational Research and Improvement. (ERIC Document Reproduction Service No. ED 368 075). 
Glines, D. \& Bingle, J. (1993). National Association for Year-Round Education: A historical perspective. California: National Association for Year-Round Education.

Glines, D. (1992). What lies ahead? Thrust for Educational Leadership, 44, 25-29.

Glines, D. (1988). YRE in California: 67 and counting. Thrust for Educational Leadership, 19, 31-34.

Glines, D. (1987). Year-Round education: A philosophy. Thrust for Educational Leadership, 16, 14-17.

Goddard, R. D. (2001). Collective efficacy: A neglected construct in the study of schools and student achievement. Journal of Educational Psychology, 93, 467-476.

Goddard, R. D. \& Goodard, Y. L. (2001, January). A multilevel analysis of the relationship between teacher and collective efficacy in urban schools. Teaching and Teacher Education, 17, 807.

Goddard, R. D., Hoy, W. K. \& Hoy, A. W. (2000). Collective teacher efficacy: Its meaning, measure, and impact on student achievement. American Educational Research Journal, 37, 479-507.

Goddard, R. D., LoGerfo, L. \& Hoy, W. K. (2004). High school accountability: The role of perceived collective efficacy. Educational Policy, 18, 403-425.

Goldman, J. P. (1990). Life's a non-stop carousel for year-round principals. The School Administrator, 74, 25-28.

Goldman, J. P. (1990). Is there really an academic payoff in year-round schooling? The School Administrator, 74, 23-25.

Goren, P. \& Carriedo, R. (1998). Policy analysis on the implementation of an expanded multi-track year-round school program. San Diego, CA: Planning Department Report of the San Diego City Schools. (ERIC Document Reproduction Service No. ED 282 328).

Grant, L. (2006) Persistence and Self-Efficacy: A Key to Understanding Teacher Turnover. Delta Kappa Gamma Bulletin, 72, 50-54.

Gresham, D. E. (2002). The relationship between teacher self-efficacy beliefs, teacher job satisfactions, socioeconomic status and student academic success. Unpublished Dissertation, Dissertation Abstracts International Section A: Humanities and Social Sciences. 
Grotjohn, D. K. \& Banks, K. (1993). An evaluation synthesis: Year-round schools and achievement. A paper presented at the Annual Meeting of the American Educational Research Association, Atlanta, GA.

Gruenert, S. \& Valentine, J. (1998). The School culture survey. Middle Level Leadership Center, University of Missouri, Columbia, MO.

Guin, K. (2004). Chronic teacher turnover in urban elementary schools. Education Policy Analysis Archive, 12(, 1-29.

Guskey, T. R. (1998). Teacher efficacy measurement and change. Paper presented at the Annual Meeting of the American Education Research Association, San Diego, CA.

Guskey, T. (1981, May). Measurement of responsibility teachers assume for academic successes and failures in the classroom. Journal of Teacher Education, 32, 44-51.

Guskey, T. R. \& Passaro, P. (1993). Teacher efficacy: A study of construct dimensions. (Paper presented at the Annual Meeting of the American Educational Research Association, Atlanta, GA. (ERIC Document Reproduction Service No. 359202.

Hackman, J. R. \& Oldham, G. R. (1974). The Job Diagnostic Survey: An instrument for the diagnosis of jobs and the evaluation of job redesign projects (Tech. Rep. No. 4). New Haven, CT: Yale University, Department of Administrative Sciences.

Hagen, K. M., Gutkin, T. B., Wilson, C. P. \& Oats, R. G. (1998). Using vicarious experience and verbal persuasion to enhance self-efficacy in pre-service teachers: 'Priming the pump' for consultation. School Psychology Quarterly, 13(2), 169 178.

Hall, D. S. (2004). Factors characterizing supportive nursing care units for registered nurses. University of Kentucky, Lexington, Kentucky. Unpublished Dissertation. UMI ProQuest Digital Dissertations.

Hall, D. T. \& Lawler, E. E. (1969). Unused potential of R. and D. labs. Research Management, 12, 339-354.

Hall, G. E., Archie, A. G. \& Rutherford, W. L. (1977). Measuring stages of concern about innovation: A manual for the use of the SOC Questionnaire. ( Report No. TM 006 654). Austin, TX: Research and Development Center for Teacher Education. ERIC Reproduction Document Service No. ED 147 342).

Hall, G. E. \& Hord, S. H. (1987). Change in schools: Facilitating the process. Albany, NY: State University of New York. 
Haser, S. G. \& Nasser, I. (2003). Teacher satisfaction in a year-round school. Educational Leadership, 60, 65-67.

Hauenstein, P. (1999). Understanding turnover. Advantage Hiring Newsletter. Retrieved December 12, 2005, from http://www.advantagehiring.com/newsletter/ n99Q4 1.html.

Haycock, I. L. (1998). Good teaching matters...a lot. Thinking K-16, 3(, 3-14. .

Hellman, C. M. (1997). Job satisfaction and intent to leave. The Journal of Social Psychology, 137, 677-689.

Hermansen, K. L. \& Gove, J. R. (1971). The year-round school: The 45 - 15 breakthrough. Connecticut: Linnet Books.

Hinkle, Wiersma \& Jurs (1988), Applied Statistics for the Behavioral Sciences, 2nd ed., Houghton Mifflin Co.

Hoi-Yan, C. (2006). The measurement of teacher efficacy: Hong Kong primary in-service teachers. Journal of Education for Teaching. 32(4), 435-451.

Hongyun, L., Lei, C. \& Quingmao, M. (2005). Teacher collective efficacy and its moderating effects on the function of teachers' self-efficacy. Acta Psychologica Sinica, 37, 79-86.

Hoy, A. W. (2000) Changes in teacher efficacy during the early years of teaching. Paper present at the annual meeting of the American Educational Research Association, New Orleans, LA, April 28, 2000.

Hoy, A. W. \& Spero, R. B. (May). Changes in teaching efficacy during the early years of teaching: A comparison of four measures. Teaching and Teacher Education, 21, 343-356.

Hoy, W. K., \& Feldman, J. A. (1987). Organizational health: The concept and its measure. Journal of Research and Development in Education, 20, 30-38.

Hoy, W. K. \& Kupersmith, W. J. (1985). The meaning and measure of faculty trust. Educational \& Psychological Research, 5, 1-10.

Hoy, W. K., \& Sabo, D. J. (1998). Quality middle schools: Open and healthy. Thousand Oaks, CA: Corwin Press, Inc.

Hoy, W. K., Smith, P. A., \& Sweetland, S. R. (2002). The development of the organizational climate index for high schools: Its measure and relationship to faculty trust. The High School Journal, 86, 38-49. 
Hoy, W. K., Smith, P. A. \& Sweetland, S. R. (2003). The development of the organizational climate index for high schools: Its measure and relationship to faculty trust. High School Journal, 86, 38.

Hoy, W. K., Tarter, C. J., \& Kottkamp, R. B. (1991). Open schools/healthy schools: Measuring organizational climate. Newbury Park, CA: Sage.

Hoy, W. K.; Smith, P. A.; Sweetland, S. R. (2002). The development of the organizational climate index for high schools: Its measure and relationship to faculty trust. High School Journal, 86, 38-39.

Hoy, W. K., \& Woolfolk, A. E. (1990). Organizational socialization of student teachers. American Educational Research Journal, 27, 279-300.

Hoy, W. K. \& Woolfolk, A.E. (1993). Teachers' sense of efficacy and the organizational health of schools. The Elementary School Journal, 93, 355-372.

Hulin, C. L., Roznowski, M., \& Hachiya, D. (1985). Alternative opportunities and withdrawal decisions: Empirical and theoretical discrepancies and an integration. Psychological Bulletin, 97, 233-250.

Ilieva, J., Baron, S., \& Healey, N. M. (2002). Online surveys in marketing research: Pros and cons. International Journal of Market Research, 44, 361-382.

Institute for Dynamic Educational Advancement. (2006). Social Cognitive Theory. Retrieved September 27, 2006, from http://www.i-d-e-a.org/pagel 10.html

Ingersol, R. M. (2005). Why do disadvantaged schools have difficulty staffing their classrooms with qualified teachers? Closing the Teacher Gap: Center for American Progress. 1-4.

Ingersoll, R. M. (2001). Teacher turnover, teacher shortages, and the organization of schools. Center for the Study of Teaching and Policy. WA: University of Washington.

Institute for Organizational Performance. (2005). Organizational climate helps predict performance. Institute for Organizational Performance.

Joffres, C. \& Haghney. (2001, March). Elementary teachers' commitment declines: Antecedents, Processes, and outcomes. The Qualitative Report, 6, 1-20.

Johnsrud, L. K. \& Rosser, V. J. (1997). Administrative staff turnover: Predicting the intention of stayers and leavers. Paper presented at the annual meeting of the Association for the Study of Higher Education, Albuquerque, NM. (ERIC Document Reproduction Service No. ED 421 936). 
Jones, L. K. (1998). Culture and conflict: A comparative study of organizations (Organizational culture, management strategies). (Doctoral Dissertation, Yeshiva University, 1998). Dissertation Abstracts International 59, 05A.

Kardos, S. M., Johnson, S. M., Peske, H. G., Kauffman, D. \& Liu, E. (2001). Counting on colleagues: New teachers encounter the professional cultures of their schools," Educational Administration Quarterly, 37, 250-290.

Keller, B. (2001). August openings public schools on hot seat. Education Week, 20, $1-25$.

Kennedy, R. L. \& Wilcher, A. E. (1998). Time and learning: Scheduling for success. Hot topics series. Phi Delta Kappan. Bloomington, IN. Center on Evaluation, Development, and Research. (ED461913).

Kenyeri, N. T. (2002). Teacher attrition: A comparison study between teacher burnout and low and high socio-economic status schools. Unpublished Dissertation. Dissertation Abstracts International Section A: Humanities and Social Sciences, 63(6-A), 2131.

Kim, I. \& Loadman, W. E. (1994). Predicting teacher job satisfaction. (ERIC Document Reproduction Service No. ED 383 707).

Klinker, J. M., Riley, D. \& Roach, M. A. (2005). Organizational climate as a tool for child care staff retention. Young Children, 60, 90-95.

Kneese, C. C. (2000). Year-round learning: A research synthesis relating to student achievement. A paper presented at the annual meeting of National Association for Year-Round Education, San Diego, CA.

Kneese, C. C. (1996). Review of research on student learning in year-round education. Journal of Research and Development in Education, 29, 60-72.

Kneese, C. C. \& Knight, S. (1995). Evaluating the achievement of at-risk students in year-round education. Planning and Changing, 26, 1-19.

Kneese, C. C. (1994). Impact of year-round education on student achievement. Unpublished Doctoral Dissertation, University of Houston, Houston, TX.

Kocek, J. 1996. The effect of year round school on teacher attendance. ERIC Document Reproduction Service No. ED 398181.

Kreis, K. (1983). The relationship between job satisfaction and needs fulfillment among urban high school teachers. (ERIC Document Reproduction Service No. ED 230 656). 
Kurz, T. B. \& Knight, S. L. (2004). An exploration of the relationship among teacher efficacy, collective teacher efficacy, and goal consensus. Learning Environments Research, 7, 111-128.

Kyriacou, C. \& Chien, P. (2004). Teacher stress in Taiwanese primary schools Journal of Educational Enquiry, 5(2), 86-104.

Lawler, E. E. III, Nadler, D. A. \& Cammann, C. (Eds.). (1980). Organizational assessment: Perspectives on the measurement of organizational behavior and the quality of work life. New York: John Wiley \& Sons.

Lee, V. E., Dedrick, R. E. \& Smith, J. B. (1991). Teacher efficacy and satisfaction. Sociology of Education, 64, 190-208.

Lester, P. E. (1987). Development and factor analysis of the Teacher Job Satisfaction Questionnaire (TJSQ). Educational and Psychological Measurement, 47, 223233.

Leung, D. Y. P. (2006). Predicting intention to quit among Chinese teachers: Differential predictability of the components of burnout. Anxiety, Stress \& Coping, 19, 129141.

Litwin, G. H. \& Stringer, R. A. Jr. (1968). Motivation and Organizational Climate. Boston: Harvard University Press.

Liu, X. S. \& Meyer, J. P. (2005). Teachers' perceptions of their jobs: A multilevel analysis of the teacher follow-up survey of 1994-95. Teachers College Record 107(5), 985-1003.

Locke, E. A. (1981). Comment on Neider: The issue of interpretation of experiments. Organizational Behavior \& Human Performance, 28, 425-430.

Loeb, S. Darling-Hammond, L. \& Luczak, J. (2005). How teaching conditions predict teacher turnover in California schools. Peabody Journal of Education, 80, 44-70.

Ma, X. \& MacMillan, R. B. (1999). Influences of workplace conditions on teachers' job satisfaction. Journal of Educational Research, 93, 39-47.

Mackenzie, S. V. (2001, April). Collective efficacy and collaborative climate in Maine high schools. Unpublished dissertation, University of Maine. Dissertation Abstracts International.

Maddox, G. H. (1998). Factors affecting teacher turnover and retention. Unpublished Dissertation, University of Colorado at Denver Graduate School of Public Affairs, Denver. 
Margolis, H. \& McCabe, P. P. (2006). Improving self-efficacy and motivation: What to do, what to say. Intervention in School \& Clinic, 41(4), 218-227.

McCaskill, E. O. \& Others. (1996). A research study about teachers' perceptions of job satisfaction. (ERIC Document Reproduction Service No. ED 184 205).

Marlow, B. H. (1993). Year-Round Education in the Harlandale Independent School District: Impact on teachers' and parents' satisfaction in selected schools. Unpublished Doctoral Dissertation, Texas A \& M University, College Station, Texas.

McCasland, C. (1992). Evaluation of H.B. Carlisle Elementary School's year-round education program: The first year. Plano, TX: Plano Independent School District.

McFadden, T. (2004). The benefits of year round education. Points of View: Year-Round School, 1-5.

(http://search.epnet.com/login.aspx?direct=true \&db=tth\&an=12462325).

McLaughlin, M. W. \& Talbert, J. E. (2003). Reforming districts: How districts support school reform: A research report. Center for the Study of Teaching and Policy, University of Washington.

Merino, B. J. (1983). The impact of year-round schooling: A review. Urban Education, 18, 299-315.

Mertler, C. A. (2001). Teacher motivation and job satisfaction in the new millennium. Paper presented at the annual meeting of the Mid-Western Educational Research Association, Chicago, IL. (ERIC Document Reproduction Service No. ED 461 649).

Minnesota Department of Children, Families \& Learning, 1999. Working group on alternative calendars: report to the legislature. National Association for YearRound Education (1999). Twenty-sixth reference directory of year-round education programs for the 1999-2000 school year. San Diego, CA.

Mizwicki, M. (1990). An analysis of the perceptions of principals, superintendents and board members in Florida towards year-round education. Unpublished Doctoral Dissertation, University of Central Florida, Orlando.

Moynihan, D. \& Pandey, S. K. (2003). Testing a Model of Public Sector Performance: How Does Management Matter? Paper prepared for the National Public Management Research Conference, October 9-11, 2003, Georgetown University.

Moore, W. P. \& Esselman, M. E. (1994). Exploring the context of teacher efficacy: The role of achievement and climate. Paper presented at the annual meeting of the 
American Educational Research Association, New Orleans, LA. (ERIC Document Reproduction Service No. ED 370 919).

Morris, V. G. and Others (1995). Parent efficacy, teacher efficacy, and parental involvement in professional development schools. Research report for Frayser Elementary School. Center for Research in Educational Policy, The University of Memphis, College of Education, Memphis, TN. (ERIC Document Reproduction Service No. ED 399 219).

Morse, S. C. (1992). The value of remembering. Thrust for Educational Leadership, 21, 35-37.

Nadler, D.A. \& Lawler, E. E. (1983). Quality of work life: perspectives and directions. Organizational Dynamics, 11, 20-30.

Norušis, M. J. (2005). SPSS 13.0 Statistical Procedures Companion. Chicago: SPSS, Inc.

Natale, J.A. (1992). Understanding home schooling. American School Board Journal, $179,26-27,29$.

National Association of Secondary School Principals. (1996). Organization and time: restructuring space and time for a more flexible education. In Breaking ranks: Changing an American Institution (pp. 45-51). VA: Reston.

National Association for Year-Round Education. (2005) Twenty-sixth reference directory of year-round education programs for the 2004-2005 school year. San Diego, California: National Association for Year-Round Education.

National Association for Year-Round Education. (1995) Year-round education: Status and possibilities. San Diego, CA: National Association for Year-Round Education.

National Center for Education Statistics. (2005, 1997). Teacher professionalism and teacher commitment: A multilevel analysis. U.S. Department of Education Office of Educational Research and Improvement, Washington, D.C.

National Education Association. (1987). What research says about: Year-round schools. Monographs of the Professional and Organizational Development/Research Division, (Number 8).

National Education Association. (1987). What research says about: Extending the school day/year: Proposals and results. Monographs of the Professional and Organizational Development/Research Division, (Number 9). 
National Education Association. (1985). A longer school day and year: Over three decades of public opinion polling. Monographs of the Professional and Organizational Development/Research Division.

Noe. R. A and Wilk S. L. 1993. Investigation of the factors that influence employees' participation in development activities, Journal of Applied Psychology, 78(2), 291-302.

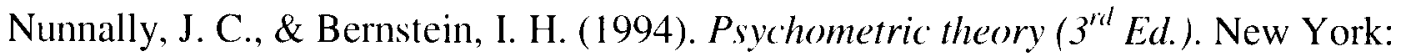
McGraw-Hill.

Nye, Konstantopoulos \& Hedges. (2004). How large are teacher effects? Educational Evaluation and Policy Analysis, 26, 237-257.

Pajares, F. (2002). Overview of social cognitive theory and of self-efficacy.

Retrieved September 27, 2006, from

http://www.emory.edu/EDUCATION/mfp/eff.html

Palmer, D. (2006). Durability of changes in self-efficacy of preservice primary teachers. International Journal of Science Education, 28(6), 655-671.

Papin, L. M. (2006). Teacher retention and satisfaction among inner-city edacators. Unpublished Dissertation. Dissertation Abstracts International Section A: Humanities and Social Sciences, 66(11-A), 3884.

Parker, L. E. (1994). Working together: Perceived self and collective efficacy at the workplace. Journal of Applied Social Psychology, 24, 43-59.

Patterson, J. H., Collins, L. \& Abbott, G. (2004). A study of teacher resilience in urban schools. Journal of Instructional Psychology. Retrieved from http://www.findarticles.com/p/articles/mi_m0FCG/is_1_31/ai_n6073183 on March, 15, 2007.

Pelavin, S. H. (1978). A study of year-round schools. Executive summary. Washington, D.C.: U.S. Department of Education, Office of Research and Improvement. (ERIC Document Reproduction Service No. ED 170 925).

Peterson, K .D. \& Brietzke, R. (2006). Reduce the cost of employee turnover and hire top performers with Profiles International. Profiles International. (http://www.profilesinternational.com/).

Picciano, A.G. (2002). Beyond student perceptions: Issues of interaction, presence, and performance in an online course. Journal of Asynchronous Learning Networks, 6, 2-19. 
Pigge, F. L. \& Marso, R. N. (1993). Outstanding teachers: Sense of teacher efficacy at four stages of career development. Paper presented at the annual meeting of the Association of Teacher Education, Los Angeles, CA. (ERIC Document Reproduction Service No. ED 356 206).

Pinkovitz, W. H. Moskal, J. \& Green, G. (2006). How much does your employee turnover cost? WI: University of Wisconsin Extension Cooperative Extension. (http://www.uwex.edu/ces/cced/publicat/turn.html).

Pittman, R. B. \& Herzog, M. J. R. (1998). Evaluation of a year-round schedule in a rural school district. Journal of Research in Rural Education, 14, 15-25. (EJ574236).

Podsakoff, P. M., MacKenzie, S. B., Lee, J., \& Podsakoff, N. P. (2003). Common method bias in behavioral research: A critical review of the literature and recommended remedies. Journal of Applied Psychology, 88, 879-903.

Poole, M. S. \& McPhee, R. D. 1983. A structurational analysis of organizational climates. In L. Putnam \& M. Pacanowsky (Eds.), Communication and organizations: An interpretive approach: 195-220. Beverly Hills, CA: Sage.

Polidor, T. P. (1996). The relation of year-round education to classroom teacher burnout. Unpublished Dissertation. Dissertation Abstracts International Section A: Humanities and Social Sciences. 56(12-A), 4628.

Prohm, B. \& Baenen, N. (1996). Are WCPSS multi-track elementary schools effective? Raleigh, NC: Wake County Public School System. ERIC Document Reproduction Service No. ED 395983.

Provasnik, S. \& Dorfman, S. (2005). Mobility in the teacher workforce NCES 204-114). U.S. Department of Education, National Center for Education statistics. Washington, D.C.: U.S. Government Printing Office.

Quality Counts. (2003). If I can't learn from you: Ensuring a highly qualified teacher for every classroom. Education Week, 22(, 459.

Quinlan, C. G. \& Emmett, T. (1987). Year-round education: Year-round opportunities A study of year-round education in California. Washington, D.C.: U.S. Department of Education, Office of Research and Improvement. (ERIC Document Reproduction Service No. ED 285 272).

Rasmussen, B. K. (1996). Perceptions of school climate, job satisfaction, stress, burnout, and their relationship to teacher absenteeism. Unpublished Dissertation. Dissertation Abstracts International Section A: Humanities and Social Sciences, 56,3402 . 
Ray, D. S. (2005). Antecedents of teacher turnover: An empirical study of the Texas public school system. Unpublished Dissertation, Dissertation Abstracts International, Nova Southeastern University.

Reese, R. (2004). The bottom line. American School Board Journal, 19, 26-27.

Riffle, M. J. S., Howley, C. W. \& Ermolov, L. D. (2004). Measure of school capacity for improvement (msci): Early field test findings. Paper presented April 14, 2004 at the 85th Annual Meeting of the American Educational Research Association, San Diego, CA.

Riggs, M. L. \& Knight, P. A. (1994). The impact of perceived group success-failure on motivational beliefs and attitudes: a causal model. Journal of Applied Psychology, 79, 755-66.

Ross, J. A., Cousins, J. B., Gadalla, T. \& Tahany, H. (1999). The effects of course assignment on teacher efficacy in restructuring secondary schools. Paper presented at the annual meeting of the American Educational Research Association, Montreal, Quebec, Canada. (ERIC Document Reproduction Service No. ED 430 280).

Ross, J. \& Gray, P. (2006). Transformational leadership and teacher commitment to organizational values: The mediating effects of collective teacher efficacy. School Effectiveness \& School Improvement, 17(2), 179-199.

Ruscoe, G. C. and Miller, S. K. (1989). Implementing a Data-Based School Effectiveness Improvement Model: Dilemmas and Lessons Learnt. Paper presented at the annual meeting of the national council of States on inservice education. San Antonio.

Russell, C. (1992). College Park Elementary School: Year-round school evaluation. Atlanta, GA: Fulton County (GA) Board of Education, Office of Planning, Research \& Development.

Sanders, P. (2001). Parent, teacher, and student satisfaction with year-round school intersession. Washington, D.C: U.S. Department of Education, Office of Educational Research and Improvement. (ERIC Document Reproduction Service No. ED458696).

Saphier, J. \& King, M. (1985). Good seeds grow in strong cultures. Educational Leadership, 42, 67-74.

Schuyler, G. (1997). A paradigm shift from instruction to learning. Washington, D.C.: U.S. Department Education, Office of Research and Improvement (ERIC Document Reproduction Service No. ED 414961). 
Schwarz, N. (1999). Self-reports: How the questions shape the answers. American Psychologist, 54, 93-105.

Serifs, D. (1990). Year-round education: A closer look. Washington, D.C: U.S. Department of Education, Office of Educational Research and Improvement. (ERIC Document Reproduction Service No. ED 329 008).

Shannon, D. M., \& Bradshaw, C. C. (2002). A comparison of response rate, response time, and costs of mail and electronic surveys. The Journal of Experimental Education, $70(2), 179-192$.

Shannon, D. M., Johnson, T. E., Searcy, S., \& Lott, A. (2002). Using electronic surveys: Advice from survey professionals. Practical Assessment. Research \& Evaluation, $8(1)$. Retrieved August 2, 2005 from http://www.unm.edu/fv3003/515Web/Shannon\%20USING\%20ELECTRONIC $\%$ 20SURVEYS.doc

Sheane, K. E. \& Others. (1994). Year-round education: Breaking the bonds of tradition. Washington, D.C.: U.S. Department of Education, Office of Educational Research and Improvement (ERIC Document Reproduction Service No. Ed 375 518).

Shields, C. (1996). Year-round education: Is it worth the hassle? Paper present at the University of British Columbia Robson Square Lecture Series. Vancouver, British Columbia, January 1996.

Shields, C. \& Oberg, S. (2000). Year-Round Schooling: Promises and Pitfalls. London: The Scarecrow Press, Inc.

Simsek, Z., \& Veiga, J. F. (2001). A primer on internet organizational surveys. Organizational Research Methods, 4, 218-235.

Smith, T. M. \& Ingersoll, R. M. (2004). What are the effects of induction and mentoring on beginning teacher turnover? American Educational Research Journal, 41, 681714.

Smith, T. M. \& Rowley, K. J. (2005). Enhancing commitment or tightening control: The function of teacher professional development in an era of accountability. Educational Policy, 19, 126-154.

Solomon, D. J. (2001). Conducting Web-based surveys, Practical Assessment, Research \& Evaluation 7(19). Retrieved on November 2002 from http://pareonline.net/getvn.asp?v=7\&n=19.

Soodak, L. C. \& Podell, D. M. (1996). Teacher efficacy: Toward the understanding of a multi-faceted construct. Teaching and Teacher Education, 12, 401-411. (ERIC Document Reproduction Service No. ED 334 434). 
Speck, M. (1996). A handbook for implementing year-round education in the high school. San Diego, CA: National Association for Year-Round Education.

Speck, M. (2002). Professional development increasing student achievement year-round education: "Why can't we get it right?" Washington, D.C.: U.S. Department of Education Office of Educational Research and Improvement. (ERIC Document Reproduction Service No. ED465709).

Steel, R. P. \& Ovalle, N. K. (1984). A review and meta-analysis of research on the relationship between behavioral intentions and employee turnover. Journal of Applied Psychology, 69, 673-686.

Stenvall, M. (1997). A checklist for success: A guide to implementing year-round schools. San Diego, CA: The National Association for Year-Round Education.

Stevens, J. (1996). Applied multivariate statistics for the social sciences. ( $3^{\text {rd }}$ Edition). Mahwah, New Jersey: Lawrence Erlbaum. Chapter 11.

Stimson, J. \& Labelle, T. J. (1971). The organizational climate of Paraguayan elementary schools: Rural-urban differentiations. Education and Urban Society, 3, 333-349.

Stolp, S. (1994, June). Leadership for School Culture. ERIC Digest (91). Retrieved December 17, 2006, from http://eric.uoregon.edu/publicutions/digests/digest091.html

Sweet, Stephen A. and Karen Grace-Martin. 2003. Data analysis with SPSS. A first course in applied Statistics. Boston, MA: Allyn and Bacon (Ch. 9 \& 10).

Tabachnick, B. G. \& Fidell, L. S. (1996). Using multivariate statistics. ( $3^{\text {rd }}$ Edition) New York: HarperCollins. Chapter 13.

Tableman, B. \& Herron, A. (2004). School climate and learning. Best Practice Briefs. University Community Partnerships, Michigan State University.

Talbert, J. M. (2002, August). "Professional communities and the artisan model of teaching." Teachers and Teaching, 8, 325-343.

Taylor, D. L. \& Tashakkori, A. (1995). Decision participation and school climate as predictors of job satisfaction and teachers' sense of efficacy. Journal of Experimental Education, 63(3), 217-203.

Tschannen-Moran, M. (2001). The effects of a state-wide conflict management initiative in schools. American Secondary Education, 29(2), 2, 31. 
Theobald, N. D. \& Michael, R. S. (2001). Teacher turnover in the Midwest: Who stays, leaves, and moves? Policy issues. Office of Educational Research and Improvement, Washington, DC.

The Southeast Center for Teaching Quality. (2004). Teacher working conditions are student learning conditions. A report to Mike Easley on the 2004 North Carolina teacher working conditions survey. Southeast Center for Teaching Quality, Chapel Hill, NC.

Thompson, D. R. (2004). Organizational learning in action: Becoming an inviting school. Journal of Invitational Theory \& Practice, 10, 52-72.

Troutman, C. S., Burke, K. G. \& Beeler, J. D. (2000). The effect of self-efficacy, assertiveness, stress, and gender on intention to turnover in public accounting. Journal of Applied Business Research, 16, 63-74.

Tschammen-Moran, M. (2000). Developing a quantitative means for assessing efficacy. Paper presented at the American Education Association, New Orleans, LA.

Tschammen-Moran, M., Woolfolk, A., \& Hoy, W.K. (1998). Teacher efficacy: Its meaning and measure. Review of Educational Research, 68, 202-248.

Tucker, E. R. (2003). The relationship between teacher morale and satisfaction, environmental robustness and sense of efficacy in an urban school district. Unpublished Dissertation, University of Kansas, Kansas.

Tuettemann, E. (1991). Teaching: Stress and satisfaction. Issues in Educational Research, 1(1), 31-42

Turan, S. (1998). A study of organizational climate and organizational commitment in human organizations. Unpublished Dissertation. Dissertation Abstracts International Section A: Humanities and Social Sciences, 59, 1038.

Vail, K. (2005). Climate control. American School Board Journal, 192, 16-19.

Venable, B. P. (1997). Year-round urban school makes news. Educational Digest, 62, 27.

Viadero, D. (2005). Teacher burnout tracked in city district. Education Week, 24, 3-16.

VanderHooven, K. (1994). Opinions regarding year-round education: A survey among the public. Washington, D.C.: U.S. Department of Education, Office of Research and Improvement. (ERIC Document Reproduction Service No. ED 376603).

Wade, M. \& Schneberger, S. (2006). Social Cognitive Theory. Retrieved September 27, 2006, from http://www.istheory.yorku.ca/socialcognitivetheory.htm 
Warrick-Harris, E. (1995). Year-round school: The best thing since sliced bread. Childhood Education, 71, 282-288.

Weiss, E. M. (1999). Perceived workplace conditions and first-year teachers' morale, career choice commitment, and planned retention: A secondary analysis. Teaching and Teacher Education, 15, 861-879. (ERIC Document Reproduction Service No. ED 598 302).

Weiss, S. (2005). The progress of education reform 2005: Teacher supply and demand. Educational Commission of the States. CO: Denver.

White, B. J. (2005). Teacher job satisfaction: A comparative study of restructured and nonrestructured elementary schools in the Philadelphia public schools. Unpublished Dissertation, Dissertation Abstracts International, St. Joseph University.

White, W. D. (1998). Year-round high schools: Benefits to students, parents, and teachers. NASSP Bulletin 72(504),103-106.

White, W. D. (1993). Educational benefits in year-round high schools. Paper presented at the Annual Meeting of the National Association for Year-Round Education, Las Vega, NV.

White, W. D. (1987). Effects of the year-round calendar on school attendance. A paper presented at the annual meeting of the National Council on Year-Round Education, Jefferson, CO.

White, W.D. (1985). Watching a district change over a decade with year-round education. Washington, D.C.: U.S. Department of Education, Office of Research and Improvement. (ERIC Document Reproduction Service No. ED 295 340).

Wildavsky, B. (1999). Scholars of summer. U.S. News \& World Report 127(5), 52-54.

Williamson, J. N. (2006). The relationship between teachers' level of social interest and teacher efficacy and their degree of job satisfaction: An exploratory study. Unpublished Dissertation. Dissertation Abstracts International Section A: Humanities and Social Sciences, 66(7-A), 2495.

Wolters, C. A. \& Daugherty, S. G. (2007). Goal structures and teachers' sense of efficacy: their relation and association to teaching experience and academic level. Journal of Educational Psychology, 99(1), 181-193.

Worthen, B. R. \& Zsiray, S. W. (1993). What twenty years of educational studies reveal about year-round education. Chapel Hill, NC: N.C. Educational Policy Center. 
Yoo, Y. (1987). Where We Stand: Comparative Study of Japanese and Kentucky Educational Requirements. Washington, D.C.: U.S. Department of Education, Office of Research and Improvement. (ERIC Document Reproduction Service No. ED280796).

Zellars, K. I., Hochwarter, W. A., Perrewe, P. I., Miles, A. K. \& Kiewitz, C. (2001). Beyond self-efficacy: Interactive effects of role conflict and perceived collective efficacy. Journal of Managerial Issues 13(4), 483-499.

Zanutto, E. (2001). Web \& E-mail Surveys. Retrieved on November 2006 from http://wwwstat.wharton.upenn.edu/ zanutto/Annenberg2001/docs/websurveys01.pdf.

Zey-Ferrell, M. (1982). Predictors of faculty intent to exit the organization: Potential turnover in a large university. Human Relations 35(5), 349-372.

Zielinski, A. E. \& Hoy, W. K. (1983). Isolation and Alienation in Elementary Schools. Educational Administration Quarterly, 19, 27-45. 


\section{APPENDICES}

\section{APPENDIX A}

Cost of Teacher Turnover and Transfer Estimates by the Alliance for Excellent Education

\begin{tabular}{rrrrrrrr}
\hline State & $\begin{array}{r}\text { Number of } \\
\text { Teachers* }\end{array}$ & $\begin{array}{r}\text { Leaving } \\
\text { Teachers }\end{array}$ & $\begin{array}{c}\text { Teacher Leaving } \\
\text { Cost }\end{array}$ & $\begin{array}{r}\text { Transferring } \\
\text { Teachers** }\end{array}$ & $\begin{array}{c}\text { Teacher } \\
\text { Transferring } \\
\text { Cost** }\end{array}$ & $\begin{array}{c}\text { Teacher } \\
\text { Turnover Cost }\end{array}$ \\
\cline { 1 - 5 } AL & 50,577 & 2,632 & $\$ 28,969,359$ & 3,815 & $\$ 41,987,258$ & $\$ 70,956,618$ \\
AK & 8,318 & 568 & $\$ 7,920,331$ & 761 & $\$ 10,611,317$ & $\$ 18,531,647$ \\
AZ & 48,088 & 3,977 & $\$ 44,026,392$ & 4,009 & $\$ 44,379,821$ & $\$ 88,406,214$ \\
AR & 30,191 & 1,434 & $\$ 14,361,155$ & 2,369 & $\$ 23,725,427$ & $\$ 38,086,582$ \\
CA & 279,945 & 14,417 & $\$ 206,213,616$ & 17,444 & $\$ 249,518,976$ & $\$ 455,732,592$ \\
CO & 42,345 & 3,637 & $\$ 41,635,928$ & 3,050 & $\$ 34,919,145$ & $\$ 76,555,073$ \\
CT & 42,122 & 2,019 & $\$ 31,359,651$ & 2,315 & $\$ 35,965,870$ & $\$ 67,325,521$ \\
DE & 7,528 & 363 & $\$ 4,841,971$ & 687 & $\$ 9,162,186$ & $\$ 14,004,157$ \\
DC & 5,708 & 426 & $\$ 6,017,796$ & 487 & $\$ 6,871,872$ & $\$ 12,889,668$ \\
FL & 128,436 & 7,152 & $\$ 78,790,723$ & 10,244 & $\$ 112,854,050$ & $\$ 191,644,744$ \\
GA & 87,839 & 6,642 & $\$ 81,736,892$ & 8,419 & $\$ 103,609,330$ & $\$ 185,346,221$ \\
HI & 12,057 & 1,282 & $\$ 15,607,820$ & 681 & $\$ 8,287,407$ & $\$ 23,895,228$ \\
ID & 14,451 & 800 & $\$ 8,530,747$ & 1,360 & $\$ 14,507,442$ & $\$ 23,038,188$ \\
IL & 137,204 & 5,662 & $\$ 78,961,817$ & 10,405 & $\$ 145,106,049$ & $\$ 224,067,866$ \\
IN & 61,135 & 2,138 & $\$ 26,843,846$ & 3,781 & $\$ 47,469,200$ & $\$ 74,313,045$ \\
IA & 38,116 & 1,882 & $\$ 20,144,334$ & 2,804 & $\$ 30,013,404$ & $\$ 50,157,738$ \\
KS & 34,134 & 2,158 & $\$ 22,649,585$ & 2,732 & $\$ 28,669,378$ & $\$ 51,318,964$ \\
KY & 42,842 & 1,650 & $\$ 18,010,556$ & 4,080 & $\$ 44,526,937$ & $\$ 62,537,493$ \\
LA & 50,806 & 3,099 & $\$ 30,776,968$ & 4,638 & $\$ 46,065,876$ & $\$ 76,842,844$ \\
ME & 17,508 & 994 & $\$ 10,606,424$ & 967 & $\$ 10,318,166$ & $\$ 20,924,590$ \\
MD & 54,553 & 3,378 & $\$ 44,644,190$ & 5,249 & $\$ 69,365,028$ & $\$ 114,009,218$ \\
MA & 78,199 & 4,011 & $\$ 56,049,714$ & 4,277 & $\$ 59,762,606$ & $\$ 115,812,320$ \\
MI & 100,221 & 4,558 & $\$ 67,056,880$ & 7,610 & $\$ 111,971,866$ & $\$ 179,028,746$ \\
MN & 57,791 & 3,315 & $\$ 39,579,507$ & 4,454 & $\$ 53,188,209$ & $\$ 92,767,715$ \\
MS & 33,009 & 1,935 & $\$ 18,492,272$ & 2,109 & $\$ 20,159,747$ & $\$ 38,652,018$ \\
MO & 64,094 & 4,036 & $\$ 43,169,611$ & 6,401 & $\$ 68,474,496$ & $\$ 111,644,106$ \\
MT & 11,921 & 573 & $\$ 5,525,286$ & 911 & $\$ 8,780,211$ & $\$ 14,305,497$ \\
NE & 23,086 & 1,120 & $\$ 11,166,635$ & 1,570 & $\$ 15,654,627$ & $\$ 26,821,262$ \\
& & & & & &
\end{tabular}




$\begin{array}{lrrrrrr}\text { NV } & 17,253 & 1,086 & \$ 12,830,603 & 2,341 & \$ 27,660,052 & \$ 40,490,655 \\ \text { NH } & 14,957 & 645 & \$ 7,299,916 & 903 & \$ 10,220,329 & \$ 17,520,245\end{array}$

APPENDIX A Continued

\begin{tabular}{|c|c|c|c|c|c|c|}
\hline State & $\begin{array}{l}\text { Number of } \\
\text { Teachers* }\end{array}$ & $\begin{array}{l}\text { Leaving } \\
\text { Teachers }\end{array}$ & $\begin{array}{c}\text { Teacher Leaving } \\
\text { Cost }\end{array}$ & $\begin{array}{l}\text { Transferring } \\
\text { Teachers** }\end{array}$ & $\begin{array}{c}\text { Teacher } \\
\text { Transferring } \\
\text { Cost** }\end{array}$ & $\begin{array}{c}\text { Teacher } \\
\text { Turnover Cost }\end{array}$ \\
\hline $\mathrm{NJ}$ & 98,310 & 4,655 & $\$ 72,633,486$ & 4,994 & $\$ 77,928,873$ & $\$ 150,562,359$ \\
\hline $\mathrm{NM}$ & 21,086 & 1,255 & $\$ 12,254,139$ & 1,601 & $\$ 15,632,756$ & $\$ 27,886,896$ \\
\hline NY & 208,278 & 13,760 & $\$ 210,614,387$ & 9,999 & $\$ 153,046,225$ & $\$ 363,660,611$ \\
\hline $\mathrm{NC}$ & 85,573 & 7,148 & $\$ 84,497,347$ & 8,804 & $\$ 104,067,934$ & $\$ 188,565,281$ \\
\hline ND & 9,246 & 398 & $\$ 3,563,447$ & 554 & $\$ 4,965,650$ & $\$ 8,529,097$ \\
\hline $\mathrm{OH}$ & 123,370 & 8,900 & $\$ 110,627,905$ & 7,708 & $\$ 95,816,606$ & $\$ 206,444,511$ \\
\hline OK & 45,739 & 2,455 & $\$ 23,047,221$ & 3,542 & $\$ 33,258,194$ & $\$ 56,305,415$ \\
\hline OR & 28,361 & 1,524 & $\$ 19,354,114$ & 2,140 & $\$ 27,179,712$ & $\$ 46,533,826$ \\
\hline PA & 126,915 & 6,100 & $\$ 88,432,504$ & 6,233 & $\$ 90,358,337$ & $\$ 178,790,841$ \\
\hline RI & 11,582 & 396 & $\$ 5,592,175$ & 772 & $\$ 10,898,365$ & $\$ 16,490,540$ \\
\hline $\mathrm{SC}$ & 43,723 & 2,822 & $\$ 30,551,316$ & 4,067 & $\$ 44,026,758$ & $\$ 74,578,074$ \\
\hline $\mathrm{SD}$ & 11,538 & 611 & $\$ 5,328,932$ & 868 & $\$ 7,569,478$ & $\$ 12,898,410$ \\
\hline $\mathrm{TN}$ & 58,275 & 2,971 & $\$ 32,378,057$ & 5,090 & $\$ 55,472,856$ & $\$ 87,850,913$ \\
\hline $\mathrm{TX}$ & 266,661 & 19,034 & $\$ 214,509,448$ & 25,768 & $\$ 290,407,937$ & $\$ 504,917,385$ \\
\hline UT & 23,346 & 1,736 & $\$ 18,203,284$ & 1,426 & $\$ 14,944,657$ & $\$ 33,147,941$ \\
\hline VT & 9,186 & 593 & $\$ 6,715,307$ & 510 & $\$ 5,773,916$ & $\$ 12,489,223$ \\
\hline VA & 80,987 & 5,337 & $\$ 62,031,275$ & 7,319 & $\$ 85,074,850$ & $\$ 147,106,125$ \\
\hline WA & 54,573 & 3,096 & $\$ 38,120,738$ & 2,996 & $\$ 36,889,448$ & $\$ 75,010,187$ \\
\hline WV & 22,552 & 636 & $\$ 6,677,984$ & 1,776 & $\$ 18,649,644$ & $\$ 25,327,629$ \\
\hline WI & 67,221 & 2,033 & $\$ 25,093,968$ & 3,114 & $\$ 38,448,836$ & $\$ 63,542,804$ \\
\hline WY & 7,839 & 393 & $\$ 4,026,798$ & 546 & $\$ 5,587,750$ & $\$ 9,614,549$ \\
\hline
\end{tabular}

\section{Total $2,998,795 \quad 173,439 \$ 2,158,074,356 \quad 220,700 \$ 2,709,805,065 \quad \$ 4,867,879,421$}

U.S. Department of Education, National Center for Education Statistics. Schools and Staffing Survey, 1999-2000. ("Public School Teacher Questionnaire," "Private School Teacher Questionnaire," and "Public Charter School Teacher Questionnaire") and 200001 Teacher Follow-up Survey ("Questionnaire for Current Teachers and Questionnaire for Former Teachers) Table 1.01. Washington, DC. 


\section{APPENDIX B \\ Year-Round Education Calendars and Plans}

45-15 Single Track. The single-track $45-15$ plan is one of the most popular calendar formats used today (Ballinger, Kirschenbaum, and Poimbeauf (1987). This type of program is divided into four nine-week terms, allow students to attend for 45 days and intersession for 15 days with a six week summer break. Four additional weeks each year are allocated to winter holidays, spring vacation, and national, state, or local holidays.

Advantages of the 45-15 calendar are that it (a) provides consistent pacing of instruction, (b) breaks up the long three-month summer vacation, and (c) allows flexible time for substituting if a teacher wants to earn pay without leaving the profession.

Disadvantages of this calendar are that it (a) does not coincide with schools using the September-June calendar and (b) requires more beginnings and endings of instructional periods than do some other YRE calendars.

The 15-day period in which students do not attend school is called "intersession." This period may be used for vacation or teachers and students may choose to spend the intersession utilizing special programs, either remedial or enrichment in nature. The programming potential of intersessions is one of the instructional advantages of the 45-15 plan.

45-15 Multi Track. According to Ballinger, Kirschenbaum, and Poimbeauf (1987), some school districts have used the YRE concept in situations where there is an over-enrollment of students for the available space. Students are normally divided into two to four groups depending on enrollment. Using the same model as the 45-15 single- 
track plan, administrators can modify it to combine several groups (tracks) of students in such a way that available space increases by 20 to $50 \%$ without building new facilities.

A four-track 45-15 plan would consist of four separate groups scheduled for nine weeks in school and three weeks on vacation, staggered from each of the other tracks. One set would be on a three-week vacation while the other three tracks respectively would be starting a term, three weeks into the term, and six weeks into the term. This plan is primarily used in overcrowding situations and allows for maximum scheduling of the school facilities.

In the 45-15 multi-track plan, students are normally divided into two to four groups, depending on enrollment. For example, in a four-track version of the plan, while groups A, B, and C are in school, group D is on vacation. When D returns, A goes on vacation. Teachers usually follow the track schedule of their students. However, they can be reassigned to another track, thereby lengthening their contract year and earning larger salaries. In addition to the advantages of the 45-15 single-track plan, two more advantages can be sited for the multi-track version: (a) can increase the school" capacity by up to $50 \%$, depending on the number of tracks used and (b) contributes to saving in operational and capital outlay costs.

Additional disadvantages are: (a) requires teachers to move from room to room if assigned to more than one track, (b) requires teachers to share classroom space and materials, and (c) requires additional planning of schedules and more communication between those on track (in school) and those off track (on vacation).

45-15 Flexible Plan. Students have the nine-week learning blocks and three-week intersession blocks as described in the 45-15 multi-track plan. However, reading, math, 
and other subjects may be individualized so those students can jump tracks for special reasons.

60-20. Ballinger, Kirschenbaum, and Poimbeauf (1987) describe this plan as a variation of the 45-15 schedule, with students attending school for 60 days and vacationing 20 days. Students complete three of these 60-day trimesters in each school year. The 60-20 plan can be conducted in either a single-track or multi-track format. This plan has become a good compromise for those who want longer teaching and vacation periods and a change from traditional calendars.

60-15. The 60-15 plan is similar to both the 45-15 and 60-20 plans in that student attend school for 60 days and vacation for 15 days. Students complete three of these 60day trimesters in each school year. Some districts schedule their 60-15 calendar school year to allow for a common three to four week summer vacation for all students. This plan can be implemented with five tracks and can be manipulated to extending the school year by three weeks over the 60-20 plan without changing the normal vacation format (Ballinger, 1987).

90-30. The 90-30 plan includes two 90-day semesters separated by 30-day vacations between terms. This type of program allows for students to have long vacations twice a year. As in the 45-15 and 60-20 plans, this calendar can be conducted as either a single-track or multi-track plan (Ballinger, 1987).

30-10 Plan. The school year is divided into eight six-week instructional blocks, with two week intersessions. Students rotate through the year until they have had six 30day school terms and six 10-day intersessions. This plan is suitable for a single-track plan or a school-within-a-school parallel system. 
Quarter Plan. The calendar is divided into four 12-week periods in fall, winter, spring, and summer. Students may select, or be assigned to, any combination of three of the four quarters. They may attend the fourth quarter on a voluntary basis, either on or off campus. The curriculum is organized so that each quarter is a separate entity. A course begins and ends with each 12-week period. For example, social studies and English programs may offer a series of separated, but related, courses. Subject areas requiring annual sequential treatment such as mathematics are offered in each of the four quarters to complete a year of work.

Trimester Plan. This plan is similar to the $60-20$ plan.

Quinmester Plan. The school year is divided into five parts with students required to attend four of the five parts. This calendar is most often used at the secondary level of grades nine through twelve. The school year may range up to 220 days, with intersessions periods averaging about seven weeks. The quinmester plan often operates on a singletrack pattern.

Five-Track, Five-Term Plan. The school year is divided into five terms of 45 days each. There are five terms in each track. Students attend for of the five for a total of 180 days of instructional use annually. This plan is usually on a multi-track basis. It provides for a common summer break of approximately three weeks for all students.

Concept 6 Plan. The Concept 6 Plan arranges six 40-44 day learning blocks; students attend four of the six and have two separate 40-44 day vacation periods; this plan provides overlapping days or longer school days to reach the 180-day requirement

Concept 8 Plan. This plan breaks the year into eight six-week terms. Students may be assigned or volunteer to attend six of the eight terms. 
Concept 16 Plan. This plan divides the year into 16 three-week terms. Students either select or are assigned to attend 12 of the 16 three-week terms.

Flexible All-Year. The Flexible All-year Plan provides for school to be open 240 days; students can select 180 days of the 240 days to attend. The curriculum is in small self-paced packages to allow for interrupted learning blocks and differentiated vacation periods - one day to several weeks at any time (Gline, 1987). 


\section{APPENDIX C}

\section{School Calendars}

School calendar variations have existed as long as the history of the United States. Throughout the mid-1800's, for example, schools in large cities were in operation eleven or twelve months of the year, although few students attended school regularly for the entire period (Glines, 1995). In contrast, most rural schools during this era were operated for only three to six months of the year. Several factors influenced the disparity in calendar arrangements. First, because eighty-five percent $(85 \%)$ of the nation's population was engaged in an agrarian lifestyle, children were needed to help with the demands of operation and maintaining family farms; second, inefficient transportation methods prohibited an easy means of transporting children to and from school; and finally, weather conditions and inadequately maintained roads prohibited many students from attending school regularly, except during the late fall and early spring when the demands of the agricultural lifestyle were lessened (Glines, 1995).

The general rationale for the length of the school calendar was determined by cultural requirements, geographic location, transportation and climate in the early days of schooling (Glines, 1995). By the turn of the century, the general population of the country had increased causing the growth of more cities and towns, and increase in urban living gradually. Transportation improved, industry flourished, and compulsory school increased with the increased number of children available to attend school. Compulsory education and the current conventional school calendar of nine months in session followed by a three-month summer vacation evolved in the United States in the mid 1800s. These initiatives provided the agricultural community a work force of children to 
help with harvesting crops. With changes in family lifestyles, both parents began to work outside the home year-round. Public opinion now increasingly supports a longer school year (Rakoff, 1999).

As a result of societal changes, additional schools were built to make education more readily available to a larger segment of the population. In addition, the organization and management of schools grew in importance and gained more social consideration. During this era of increased social consideration, the length of the school calendar generally decreased in cities and increased in rural areas. This blending of the urban and rural calendar created a degree of standardization in the length of the school year. In spite of these standardizing changes, however, remarkable variations in the length of the school calendar continued to exist across the country, within states and local school districts (Glines, 1995).

Historians have reported that innovative calendars existed within a number of schools during this same time period. Summer 'vacation schools' and schools with multitrack calendars were designed to accommodate overpopulation, thereby becoming the forerunners of the concept of YRE. Later, during the decades prior to World War II, a greater assortment of calendar schemes came into existence (Glines, 1995). During the war effort, however, emphasis on national unity brought about the demise of the YRE trends and stimulated the emergence of our current September to June, 170-180 day school agrarian calendars. Time accepted general rules of designing school calendars dictate that school district officials may not schedule days of session on a Saturday or a Sunday or a legal holiday. Legal holidays may include: New Year's Day, Dr. Martin 
Luther King, Jr. Day, President's Day, Memorial Day, Independence Day, Labor Day, Veteran's Day, Thanksgiving Day and Christmas Day.

Despite economic and family changes and the greater knowledge and more complex skills demanded of $21^{\text {st }}$ Century workers, schools and districts continue to use time as they did when students" after-school activities included "chopping and toting firewood" and weeding crops (Ballinger, 1999, 1998, 1997). At the same time, other countries have been making more time available for learning and using the available time differently through increased days and increased hours in the school day. Most schools in America still operate on a September - May 10-month system. When the United States gradually moved from an agrarian economy to an industrialized economy, schools still remained overall unchanged (Goldberg \& Cross, 2005): a) Schools open and close their doors at fixed times in the morning and early afternoon - 8:30-3:00; b) The school year lasts nine months, beginning in late summer after labor Day and ending in spring around Memorial Day; c) Schools typically offer a six-period day, with about six hours of classroom time a day; d) No matter how complex or simple the school subject literature, shop, physics, gym, or algebra - the schedule assigns each school course an impartial national average of 51 minutes per class period, despite how well or poorly students may comprehend the material; e) The norm for required school attendance, according to the Council of Chief State School Officers, is 180 days. Eleven states permit school terms of 175 days or less; only one state requires more than 180 days; f) Secondary school graduation requirements are universally based on seat time, in the form of Carnegie units, a standard of measurement representing one credit for completion of a one-year course meeting daily; g) Teaching staff salary increases are typically tied to 
time - to seniority and the number of hours of graduate work completed; and h) Despite the obsession with time, little attention is paid to how it is used: In forty-two states, only forty-one percent of secondary school time must be spent on core academics. For the United States to stay in competition with the global community it is vital to revisit how much time is devoted to learning over the course of a lifetime and how that time is spent (Goldberg \& Cross, 2005).

The widespread concern that American schools are not serving up a quality education for all students has been fueled in part by international comparisons of student achievement, which seem to show American students lagging behind their counterparts in other leading industrialized nations (Anoff, 1999). Some of these same studies also indicate that American students spend considerably less time in school than those in some of the countries that outperform us (Baird, 2005). That apparent correlation of time and achievement reinforces a common assumption that when it comes to time in education, more is better. If the American school year or day were longer, the theory goes, our students would learn more. Some policy makers seem to be betting on it. However, as part of the thrust to reform schools, states have legislated high-stakes testing and mandated increased instructional hours. To maximize preparations for state assessments, some school districts are starting classes earlier in August or July. Other districts have added days to accommodate a state-mandated expansion of hours, while others modify the calendar for localized reasons (Keller, 2001). Some of these modified calendars add days, usually as remedial intersessions between breaks. Summer sessions also play an increasing role. For these and other reasons, more schools across the country are altering instructional schedules. Several rural districts are adopting four-day weeks for financial 
reasons. Other schools are experimenting with trimesters. Extended learning schedules for students with academic deficiencies are now available in many districts (Keller, 2001).

\section{School Calendars around the World and the YRE Calendar}

Economics and economic competition from Japan and Europe are forcing education restructuring. Nations with the best educated citizenry are the ones most likely to survive in international competition (Kemp, 1995). Comparisons with Japan and other countries with the highest achievement scores have led some commentators to attribute such differences to a longer school calendar, e.g., 240-260 days in length (Weiss, 1999). Barrett (1990) provides a chart depicting a comparison of school days per year for countries around the world in Table 11.

\section{Table 11}

Length of School Calendars around World

\begin{tabular}{l|l|c}
\hline \multicolumn{2}{l|}{ Country } & Days \\
\hline Japan & New Zealand & 243 \\
West Germany & Nigeria & $226-240$ \\
South Korea & British Columbia & 220 \\
Israel & France & 216 \\
Luxembourg & Ontario & 216 \\
Soviet Union & Ireland & 211 \\
Netherlands & New Brunswick & 200 \\
Scotland & Quebec & 200 \\
Thailand & Spain & 200 \\
Hong Kong & Sweden & 195 \\
England/Wales & United States & 192 \\
Hungary & French Belgium & 192 \\
Swaziland & Flemish Belgium & 191 \\
Finland & & 190 \\
& & \\
\hline
\end{tabular}


Closer inspection of school time reveals that Japanese students attend schools on Saturday. Issues surrounding length of school day are complex, as they relate to time zones, weather conditions, busing schedules, coordination with other timetables, work, and the like (Weiss, 1999). Politicians and the general public are looking at education and wondering why schools, which cost millions of dollars, are kept closed for almost three months in a year while educators complain about overcrowding in Canadian schools (Weiss, 1999).

Yoo (1987) reports that on tests given to students in Australia, Canada, England, France, Japan, Sweden, Switzerland and two Dallas schools, Japanese won the highest place in mathematics while the United States finished last and furthermore, finished last in seven of nineteen tests administered while ranking first or second on none. Yoo summarized weekly minimum instructional requirements and total hours per year for each grade level in Japan and Kentucky schools. The summary reports that Kentucky requires more hours per week but actually fewer for the school year. The reason for this difference in instructional hours is that the minimum number of instructional days for Kentucky is 175 while Japan requires 240 days (Barrett, 1990). In Kentucky, classes are typically conducted only for five days a week, Monday through Friday. However, Japan has six days of classes, Monday through Saturday. Following this school days requirement, during the first two grades Japan teaches its pupils for an additional 150 hours per year (Yoo, 1987). From grades three through six, Japanese students spend an additional 300 hours over and above hours spent by Kentucky students in their classes.

Already established as spending more hours per year in instruction, many Japanese students are enrolled in private tutoring programs after school, the "Katushu 
Juku," nicknamed "Juku" (Yoo, 1987). About sixty percent of Japanese public school students and ninety percent of Japanese private school enrollees participate in these programs. After the sixth grade, Japanese students must pass an entrance exam before entering middle school (grades 7-9) and pass another entrance exam before high school (grades 10-12). Because of these entrance exams, students are highly concerned about doing their best.

The "taken-for-granted" nature of the traditional school year in the United States appears to convert to passionate defense of its merits when some communities are confronted with the possibility of change. "In conducting research in school districts on policy deliberations about year-round education, my major conclusion was that most parents and other community members are not open to even contemplation of the issue" (Weis, 1995, p. 5). This aversion to contemplation of YRE results from engrained resistance to change, change that would interrupt the rhythms of the other parts of people's lives, namely work, leisure, daycare and other social institutions (Weis, 1995). Weis goes on to state that,

"In the larger community...we have organized ourselves over 150 years so that only during the summer months do we have to accommodate large numbers of children on the loose. We have built entire economic and social structures for that purpose- which have implications for how local government and law-enforcement functions, on the economies of scale of maintaining schools, on providing buses for schools, on child specific industries such as summer camps, on economic operations such as small business that depend on students for summer help" (Weis, 1995, pp. 7-8). 
Not only has YRE been a state and national time school calendar reform, it also has been a school calendar reform on the international scale. Research and studying of the concept of YRE started in Canada as early as 1971 in Alberta, 1972 in Ontario, and 1988 in Saskatchewan (Kemp, 1995). Like most YRE implementation within the United States, YRE started in British Columbia with one school, three schools in Alberta, one in Manitoba, and one in Ontario. By September 2005, YRE enrollment in Canada reached 12,070 students. Most difficulties arose from convincing stakeholders that the change from the traditional to a seldom-tried calendar like YRE is needed to do something better for children, parents, staff, school and community (Kemp, 1995).

YRE around the world differs slightly from the YRE approach in the United States. YRE is different in that in the U.S. educators take the basic 170-184 instructional day school year (depending on the state) and stretch them out in such a way that no vacation period is longer than eight weeks (NAYRE's outer limit to be called a YRE school). In Europe, Asia and Australia, where the school year is 200-240 instructional days a year (again, depending on the country) no vacation is longer than eight weeks because one cannot fit in all the instructional days and still have eight weeks or more of vacation at any one time. Indeed, in some countries, six weeks is more the norm for summer vacation (Ballinger, 2004).

\section{Introduction to the YRE}

\section{Definitional/Conceptual Problem of YRE}

When discussing YRE, a conceptual problem immediately occurs. Students and teachers have a vision of attending school all year - increased days and fewer vacation breaks. However, YRE requires no greater number of days. The distribution of days in 
school and days on break on the nine-month traditional agrarian calendar and the distribution of school days on a YRE balanced or modified calendar determine the major differences in the calendar approach. Weekends are excluded, with both calendar models detailing a typical year of 258 work days (Monday through Friday). Both calendars represent a standard school year of 180 days (175 in Kentucky).

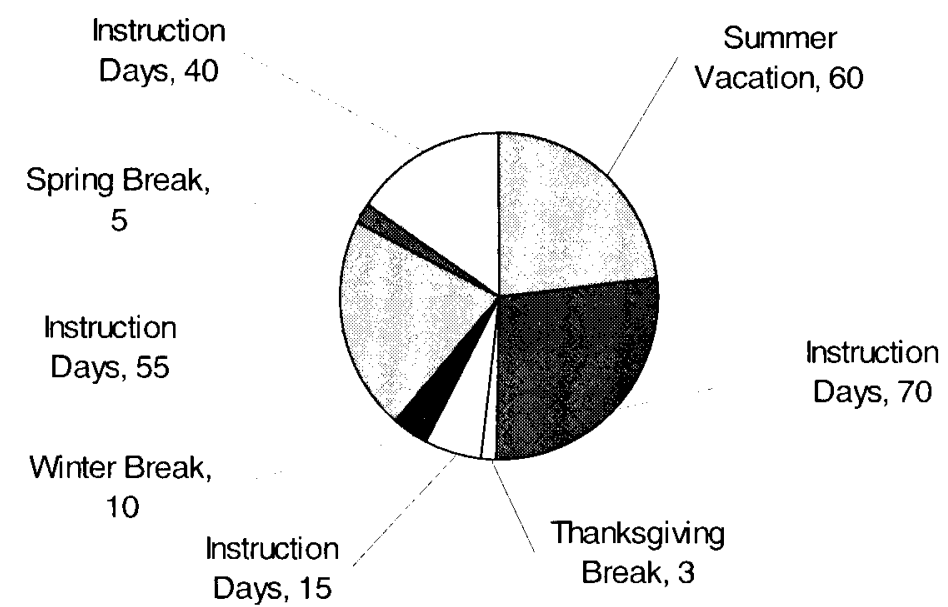

Figure 7. Traditional School Calendar: Vacation and instructional days

The traditional calendar (See Figure 7) features a long summer vacation of twelve weeks followed by a long period of in-session instructional days, with the first break occurring at Thanksgiving. The winter holidays consist of fifty-five in-session instructional days followed by a short spring break. Spring break is followed by forty insession instructional days before the end of the school year (NAYRE, 2006). 


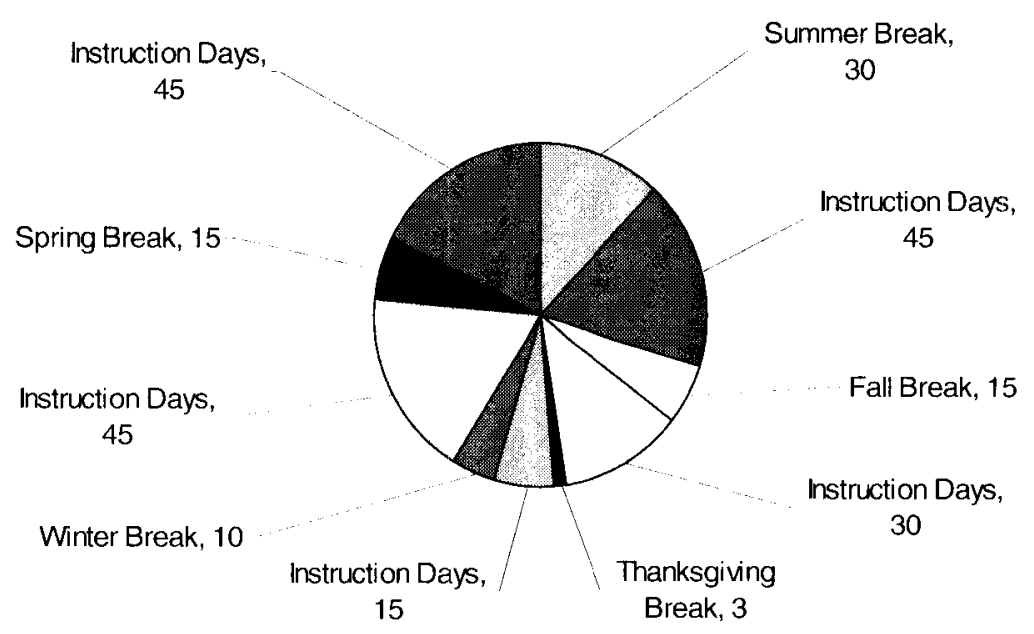

Figure 8. Year-Round Education School Calendar: Vacation and instructional days.

The YRE calendar (See Figure 8) reduces the long summer break and simply reapportions those days throughout the school year, producing more frequent breaks and thus limiting long periods of in-session instructional days, as well as longer vacations. Although both calendars feature 180 days of instruction, the YRE modified calendar balances the frequency of in-session instructional days with days on break. The winter holiday and Thanksgiving break can be the same on both calendars (NAYRE, 2006).

Although many YRE calendars exist with variations in the distribution of instructional and vacation days (see Appendix B), this study will only explore the concept of single-track modified calendars. YRE calendars, often referred to as balanced (NAYRE, 2006) calendars, are designed to best meet the needs of the school or school district. Schools and districts may design different variations of the YRE calendar in the context of vision and mission. Some variations of YRE calendars embrace school all year round with alternating in-school sessions and vacations. Modified YRE school calendars allow for summer vacations greater than three weeks and less than eight weeks. Alternating forty-five (45) in-school session and fifteen (15) day vacations determine the 
schedule during the rest of the calendar year. The scope of this study will explore the possible influence that YRE calendar reform might have on school climate, school culture, teacher efficacy, collective efficacy, teacher job satisfaction and teacher intent to turnover. Single track modified YRE calendars provide a balanced calendar for a more continuous period of instruction as well as vacation breaks for both students and teachers (Ballinger, 1997). Students and staff follow the same instructional and vacation schedule (Ballinger, 1999). Single-track does not reduce class size, nor does it allow a school to accommodate more students. The long summer vacation is shortened with additional vacation days distributed throughout the school year into periods called "intersessions" (See definitions). Intersessions allow time for instructional remediation and enrichment throughout the school year. The most common types of single-track calendars are 45-15, 60-20 and 90-30 (Ballinger, 1997, 2000; See Appendix B).

For teachers, the YRE calendar is an employment schedule change - reassignment of work and vacation days. YRE advocates suggest the reassignment of instructional and vacation days has a cumulative effect on teachers regarding working conditions, school climate, school culture, teacher efficacy, collective efficacy and teacher job satisfaction (Ballinger, 1997, 2000). Yes, little research has examined how and why these variables influence teacher intent to turnover in the YRE calendar context.

\section{The History of YRE}

\section{Early Experimentation with YRE}

To understand fully the existing YRE calendar reform, it is important to review the past. In rural areas in the United States, students were needed on the farm during spring, summer and fall. Historically, rural schools operated for five or six months, from 
the last harvest to the first planting (Glines, 1995; Glines \& Bingle, 1993). Conditions in rural areas were different as no big organizations lobbied for longer school years or for compulsory attendance legislation (Glines, 1995; Glines \& Bingle, 1993). The four main reasons for the short school year in rural America were:

1. The need for child labor on the farm;

2. Poor roads made traveling extremely difficult;

3. Lack of money often led to early school closings; and

4. Failure to see the value of a good education (Glines, 1995).

Many urban areas had eleven or twelve-month school calendars. Merino (1983) reported that in the nineteenth century a number of school districts operated throughout the year. Buffalo, for example, had a twelve-month calendar. Brooklyn, Baltimore, Cincinnati, and Chicago had eleven-month calendars. In the mid 1800s, New York City was on a fortynine week schedule; Chicago held classes for forty-eight weeks, and Cleveland for fortythree weeks (Hermansen \& Gove, 1971). In the early 1900s, longer school calendars existed in Bluffton, Indiana (1904); Amarillo, Texas (1910); Newark, New Jersey (1912); Minot, North Dakota (1917); Omaha, Nebraska (1922); Nashville, Tennessee (1924); Aliquippa, Pennsylvania (1928); and Ambridge, Pennsylvania (1932). Other short-lived pre-World II YRE programs included Bayonne, New Jersey; Albuquerque, New Mexico; Ardmore and Tulsa, Oklahoma; Eleveth, Minnesota; and Mason City, Iowa. Several large cities during the era were cited in the literature with limited details: Cleveland, Minneapolis, Detroit, and New York City (Glines, 1995; Glines \& Bingle, 1993). Other YRE pioneers included Henri Weber, Harlan Vanderslice, John Beveridge, Addison Poland, Warren Roe, and Bennet Jackson (Glines, 1995). 
World War II brought a push toward uniformity in education as the symbol of society in the United States (Glines, 1995). Because of the war, until the end of the hostilities in the late 1940s, all experimentation with YRE had slowed (Glines, 1995; Glines \& Bingle, 1993). In the late 1960s and the early 1970s, the YRE concept would be reactivated by a new group of school districts as they adopted the YRE programs, including Haywood (California), Valley View and Romeo (Illinois), Virginia Beach (Virginia), St. Charles (Missouri), and Phoenix (Arizona). Mizwicki (1990) noted community concerns that caused reactivation or renaissance in the YRE concept as unbalanced student population growth patterns, elected officials less willing to expand their financial support, change in the national economy due to increased taxes and the pressures of supporting the Vietnam War (Glines, 1995; Glines \& Bingle, 1993).

After World War II and up through the 1970's, the majority of school districts considering YRE initially did so to increase the capacity of existing facilities, reduce the need for new construction, and save money (Glines, 1988). Since the 1970's, a growing number of school communities are considering other factors such as: continuity of learning, curriculum enhancement, and the concept that schools, like other institutions, should never be closed. Each year many new communities were added to the roster of YRE schools as single-track calendars. Table 12 presents the current growth of YRE in the nation and around the world (NAYRE, 2006). 
Table 12

National Summary of Year-Round Education, 2005-2006

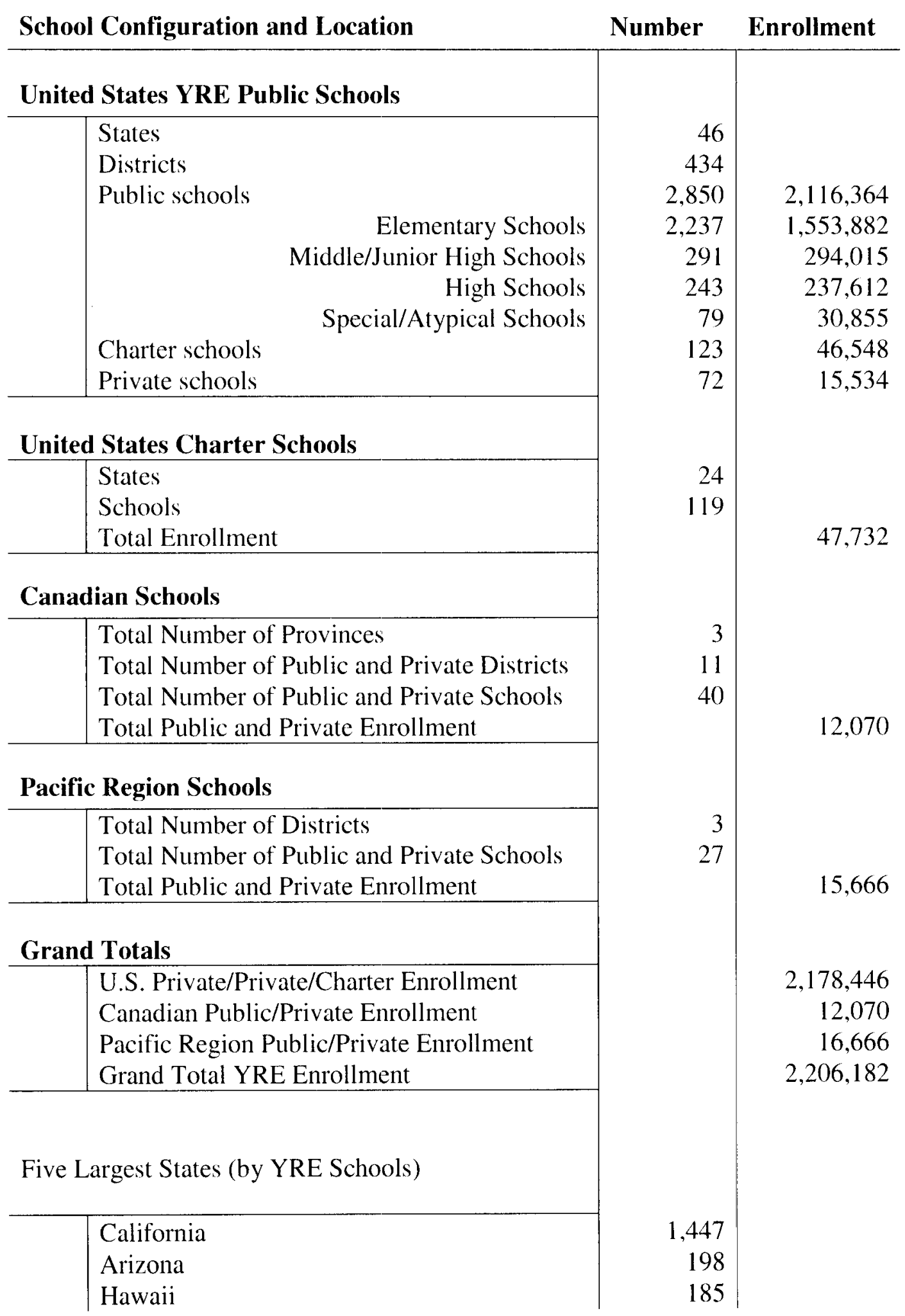




\begin{tabular}{|c|c|c|}
\hline \begin{tabular}{l|l} 
Kentucky \\
Texas
\end{tabular} & \multirow[t]{2}{*}{$\begin{array}{r}115 \\
99\end{array}$} & \multirow{5}{*}{$\begin{array}{r}1,264,058 \\
138,813 \\
115,633 \\
79,142 \\
61,782\end{array}$} \\
\hline Five Largest States (by Enrollment) & & \\
\hline $\begin{array}{l}\text { California } \\
\text { Arizona } \\
\text { Hawaii } \\
\text { Nevada } \\
\text { Kentucky }\end{array}$ & & \\
\hline Five Largest States (YRE Districts) & & \\
\hline \begin{tabular}{|l} 
California \\
Arizona \\
North Carolina \\
Texas \\
Kentucky
\end{tabular} & $\begin{array}{r}172 \\
46 \\
34 \\
32 \\
29\end{array}$ & \\
\hline
\end{tabular}

*Twenty-Ninth Reference Directory of Year-Round Education Programs for the 20052006 School Year: NAYRE.

According to NAYRE (2006), Table 12 reports the number of states involved in YRE for the 2005-2006 school year was forty-six. This included 434 districts with a total of seventy-nine special/atypical schools, 2,237 elementary schools, 291 middle schools, and 243 high schools. The five states with the most involvement (by YRE enrollment) were California, Arizona, Hawaii, Nevada, and Kentucky. The five states with the greatest number of YRE schools are California, Arizona, North Carolina, Texas, and Kentucky. According to Harp (1994), the number of students in schools using YRE calendars is up for the fifteenth year. While the national trend in YRE is on steady increase, Ballinger (1999) states that Kentucky has exploded with interest in YRE with zero schools in 1993-94 to 103 schools in 2005-2006. 


\section{APPENDIX D}

Organizational Climate Research Instruments

\begin{tabular}{|c|c|c|c|}
\hline Instrument/Scale & Authors & $N$ & $\begin{array}{l}\text { Scale and/or Subscale } \\
\text { Reliability }\end{array}$ \\
\hline $\begin{array}{l}\text { Organizational } \\
\text { Climate Index }\end{array}$ & $\begin{array}{l}\text { Hoy, Smith \& } \\
\text { Sweetland, } 2002\end{array}$ & $N=97$ & $\begin{array}{l}\text { institutional } \\
\text { vulnerability, .87; } \\
\text { collegial leadership, } \\
.94 ; \text { professional } \\
\text { teacher behavior, .88; } \\
\text { achievement press, } 92\end{array}$ \\
\hline $\begin{array}{l}\text { Comprehensive } \\
\text { Assessment of } \\
\text { School } \\
\text { Environments } \\
\text { (CASE) }\end{array}$ & $\begin{array}{l}\text { National } \\
\text { Association of } \\
\text { Secondary } \\
\text { School } \\
\text { Principals, } 1986\end{array}$ & $N=1500$ & $\begin{array}{l}\text { Cronbach's alpha } \\
\text { subscale average is } \\
.88 \text {, with a range from } \\
.80 \text { to } .93\end{array}$ \\
\hline $\begin{array}{l}\text { Organization Health } \\
\text { Inventory }(\mathrm{OHI})\end{array}$ & $\begin{array}{l}\text { Hoy \& Sabo, } \\
1998\end{array}$ & $N=1131$ & $\begin{array}{l}\text { institutional integrity, } \\
.91 ; \text { initiating structure, } \\
.89 ; \text { consideration, .90; } \\
\text { principal influence, } \\
.87 ; \\
\text { resource support. } .95 ; \\
\text { morale. } .92 ; \\
\text { academic emphasis, } \\
.93\end{array}$ \\
\hline $\begin{array}{l}\text { Organizational } \\
\text { Climate Descriptive } \\
\text { Questionnaire } \\
\text { (OCDQ) }\end{array}$ & $\begin{array}{l}\text { Hoy \& Sabo, } \\
1998\end{array}$ & $N=38$ & $\begin{array}{l}\text { supportive principal } \\
\text { hehavior, .95; directive } \\
\text { principal behavior, } 89 \text {; } \\
\text { restrictive principal } \\
\text { hehavior, .80; collegial } \\
\text { teacher behavior, } .90 ; \\
\text { intimate teacher } \\
\text { behavior, } .85 ; \\
\text { disengaged teacher } \\
\text { behavior, } .75\end{array}$ \\
\hline $\begin{array}{l}\text { Organizational } \\
\text { Climate Description } \\
\text { Questionnaire }\end{array}$ & $\begin{array}{l}\text { Halpin \& Croft, } \\
1963\end{array}$ & $N=218$ & $\begin{array}{l}\text { Cronbach's alpha }= \\
0.90 \text { and } 0.85 \text { using } \\
\text { test-retest method }\end{array}$ \\
\hline
\end{tabular}




\begin{tabular}{|c|c|c|c|}
\hline $\begin{array}{l}\text { Organizational } \\
\text { Climate Description } \\
\text { Questionnaire- } \\
\text { Rutgers Secondary } \\
\text { (OCDQ-RS }\end{array}$ & Turan, 1998 & $N=900$ & $\begin{array}{l}\text { Cronbach's alpha } \\
\text { coefficients ranged } \\
\text { from } .91 \text { to } .71\end{array}$ \\
\hline $\begin{array}{l}\text { Organizational } \\
\text { Commitment } \\
\text { Questionnaire }\end{array}$ & $\begin{array}{l}\text { Mowday et al., } \\
1979\end{array}$ & $N=2563$ & $\begin{array}{l}\text { Cronbach's alpha } \\
\text { coefficients ranged } .82 \\
\text { to } .93\end{array}$ \\
\hline $\begin{array}{l}\text { Organizational } \\
\text { Climate Index: } \\
\text { Dimensions of } \\
\text { Organizational } \\
\text { Climate (OCI) }\end{array}$ & $\begin{array}{l}\text { The Institute for } \\
\text { Organizational } \\
\text { Performance, } \\
2005\end{array}$ & $N=395$ & $\begin{array}{l}\text { Cronbach's alpha for } \\
\text { subscales } \\
\text { accountability, .88; } \\
\text { collaboration, .88; } \\
\text { leadership, .93; } \\
\text { alignment } .83 ; \\
\text { adaptability, .63; } \\
\text { dimension of trust, } .87\end{array}$ \\
\hline
\end{tabular}




\section{APPENDIX E}

Organizational Culture Research Instruments

\begin{tabular}{|c|c|c|c|}
\hline Instrument/Scale & Designers/Authors & $N$ & Reliability \\
\hline $\begin{array}{l}\text { School Culture } \\
\text { Indicators Scale }\end{array}$ & $\begin{array}{l}\text { McLaughlin \& } \\
\text { Talbert, } 2003\end{array}$ & $N=56$ & $\begin{array}{l}\text { Cronbach's alpha for } \\
\text { subscales: } \\
\text { inquiry practices, } .86 \text {; } \\
\text { teacher learning, .84; } \\
\text { collective problem, .83 }\end{array}$ \\
\hline $\begin{array}{l}\text { School Culture } \\
\text { Survey }\end{array}$ & $\begin{array}{l}\text { Saphier \& King, } \\
1985\end{array}$ & $N=425$ & $\begin{array}{l}\text { Cronbach's alphas for } \\
\text { subscales: professionalism } \\
\text { among teacher, } .91 ; \\
\text { professional treatment by } \\
\text { administration, } .86 ; \\
\text { collaboration, } .81\end{array}$ \\
\hline $\begin{array}{l}\text { Organizational } \\
\text { Culture Assessment } \\
\text { Inventory }\end{array}$ & $\begin{array}{l}\text { Steinhoff \& Owens, } \\
1988\end{array}$ & $N=56$ & \\
\hline $\begin{array}{l}\text { Effective School } \\
\text { Function Inventory }\end{array}$ & $\begin{array}{l}\text { Carter \& Michael, } \\
1995\end{array}$ & $N=124$ & $\begin{array}{l}\text { Internal consistency } \\
\text { Cronbach's alpha reported to } \\
\text { be between } .60 \text { and } .75\end{array}$ \\
\hline $\begin{array}{l}\text { CTP Teacher } \\
\text { Survey Scale }\end{array}$ & $\begin{array}{l}\text { Center for the Study } \\
\text { of Teaching and } \\
\text { Policy, } 2002\end{array}$ & $N=2594$ & $\begin{array}{l}\text { Cronbach's alphas for } \\
\text { subscales: improving teaching } \\
\text { and learning, .84; } \\
\text { developing the profession. .74; } \\
\text { allocating resources, } .70 ; \\
\text { communicating within and } \\
\text { beyond, } .73 ; \text { creating local } \\
\text { accountability, .72; partnering } \\
\text { with nonsystem actors, .80; } \\
\text { school learning community, } \\
.89 ; \text { distributed leadership, } \\
.72 ; \text { sustainability, .57; low } \\
\text { constraints on school hiring, } \\
.88) ; \text { top-down culture, } .52 ; \\
\text { pride, .81 }\end{array}$ \\
\hline
\end{tabular}




\begin{tabular}{l|l|l|l}
$\begin{array}{l}\text { School Culture } \\
\text { Survey }\end{array}$ & $\begin{array}{l}\text { Gruenert \& } \\
\text { Valentines, 1998 }\end{array}$ & $N=632$ & $\begin{array}{l}\text { Cronbach's alpha for } \\
\text { subscales: collaborative } \\
\text { leadership, } .91 ; \text { teacher } \\
\text { collaboration, .83; } \\
\text { unity of purpose, .82; } \\
\text { professional development, } .87 ; \\
\text { and learning partnership, } .80\end{array}$ \\
$\begin{array}{l}\text { Michigan } \\
\begin{array}{l}\text { Organizational } \\
\text { Assessment } \\
\text { Questionnaire }\end{array}\end{array}$ & $\begin{array}{l}\text { Cammann, } \\
\text { Fichman, } \\
\text { Jenkins and } \\
\text { Klesh 1979; }\end{array}$ & $N=518$ & Cronbach's alpha $=.87$ \\
$\begin{array}{l}\text { Collective Efficacy } \\
\text { Scale }\end{array}$ & $\begin{array}{l}\text { Riggs \& Knight, } \\
1994\end{array}$ & $N=80$ & Cronbach's alpha $=.76$ \\
\hline
\end{tabular}




\section{APPENDIX F}

Teacher Efficacy Research Instruments

\begin{tabular}{|c|c|c|c|}
\hline Instrument/Scale & Designers/Authors & $N$ & $\begin{array}{c}\text { Reliability } \\
\text { Scale/Subscales }\end{array}$ \\
\hline $\begin{array}{l}\text { Teachers' Sense of } \\
\text { Teacher Efficacy } \\
\text { Scale }\end{array}$ & $\begin{array}{l}\text { Tschannen-Moran } \\
\& \text { Hoy, } 2001\end{array}$ & $\begin{array}{l}3 \text { studies } \\
N=224 \\
N=217 \\
N=183\end{array}$ & $\begin{array}{l}\text { Cronbach's alpha: } \\
\text { total scale, } .94 ; \\
\text { engagement, } .87 \\
\text { instruction, } .91 ; \\
\text { management, } .90\end{array}$ \\
\hline $\begin{array}{l}\text { Teacher Efficacy } \\
\text { Scale }\end{array}$ & $\begin{array}{l}\text { Dembo \& Gibson, } \\
1984\end{array}$ & & $\begin{array}{l}\text { Cronbach's alpha: } \\
\text { total scale, .79; } \\
\text { personal teaching } \\
\text { efficacy, .78; } \\
\text { teaching efficacy, } .75\end{array}$ \\
\hline $\begin{array}{l}\text { Teacher Efficacy } \\
\text { Scale }\end{array}$ & $\begin{array}{l}\text { Hoy \& Woolfolk, } \\
1990\end{array}$ & $N=191$ & $\begin{array}{l}\text { Cronbach's alpha, were } \\
0.76 \text { for the five items } \\
\text { related to personal } \\
\text { teaching efficacy and } \\
0.78 \text { for the five items } \\
\text { related to general } \\
\text { teaching efficacy. }\end{array}$ \\
\hline $\begin{array}{l}\text { Responsibility for } \\
\text { Student } \\
\text { Achievement }\end{array}$ & Guskey, 1981 & $N=120$ & $\begin{array}{l}\text { Cronbach's alpha } \\
\text { reliability coefficients }= \\
.79 \text { and } .88 \text {, respectively }\end{array}$ \\
\hline $\begin{array}{l}\text { Teacher Locus of } \\
\text { Control }\end{array}$ & $\begin{array}{l}\text { Rose \& Medway, } \\
1981\end{array}$ & $N=44$ & \\
\hline $\begin{array}{l}\text { Webb Efficacy } \\
\text { Scale }\end{array}$ & $\begin{array}{l}\text { Ashton \& Others, } \\
1982\end{array}$ & $N=48$ & $\begin{array}{l}\text { correlations between } \\
\text { efficacy and stress } \\
\text { ranging from - }-05 \text { to - } \\
.82 \text {, with an average of - } \\
.39\end{array}$ \\
\hline
\end{tabular}




\begin{tabular}{|c|c|c|c|}
\hline $\begin{array}{l}\text { Teacher Efficacy } \\
\text { Scale }\end{array}$ & Bandura, 1997 & $N=540$ & Cronbach's alpha $=.92$ \\
\hline $\begin{array}{l}\text { Purdue Teacher } \\
\text { Opinionnaire }\end{array}$ & $\begin{array}{l}\text { Bentley \& } \\
\text { Rempel, } 1980\end{array}$ & $N=3023$ & $\begin{array}{l}\text { Cronbach's alpha: } \\
\text { teacher rapport with } \\
\text { principal, } .96 ; \\
\text { satisfaction with } \\
\text { teaching, .88; rapport } \\
\text { among teachers, } .94 ; \\
\text { teacher salary, } .74 ; \\
\text { teacher load, } .79 ; \\
\text { curriculum issues, } .73 ; \\
\text { teacher status, } .82 ; \\
\text { community support of } \\
\text { education, .78; school } \\
\text { facilities and services, } \\
\text {.69; community } \\
\text { pressures, .55 }\end{array}$ \\
\hline $\begin{array}{l}\text { Environmental } \\
\text { Robustness } \\
\text { Semantic } \\
\text { Differential Scale }\end{array}$ & $\begin{array}{l}\text { Licata \& } \\
\text { Willower, } 1978\end{array}$ & & Cronbach's alpha $=.87$ \\
\hline $\begin{array}{l}\text { Schools and } \\
\text { Staffing Survey } \\
\text { (SASS }\end{array}$ & $\begin{array}{l}\text { National Center } \\
\text { for Educational } \\
\text { Statistics, } 2005\end{array}$ & $N=52,000$ & $\begin{array}{l}\text { Cronbach's alpha: } \\
\text { participation in } \\
\text { decision making , } .80 ; \\
\text { administrative support, } \\
.86 ; \text { collaborative } \\
\text { atmosphere, } .61 ; \text { social } \\
\text { climate, } .92\end{array}$ \\
\hline $\begin{array}{l}\text { Teacher Efficacy } \\
\text { Scale }\end{array}$ & $\begin{array}{l}\text { Soodak \& Podell, } \\
1996\end{array}$ & $N=310$ & Cronbach's alpha $=.79$ \\
\hline $\begin{array}{l}\text { Teacher Efficacy } \\
\text { Scale }\end{array}$ & $\begin{array}{l}\text { Ross, Cousins, } \\
\text { Gadalla, \& } \\
\text { Tahany, } 1999\end{array}$ & $N=359$ & Cronbach's alpha $=.86$ \\
\hline $\begin{array}{l}\text { Teacher Efficacy } \\
\text { Scale }\end{array}$ & $\begin{array}{l}\text { Ruscoe \& Miller, } \\
1989\end{array}$ & $N=1500$ & $\begin{array}{l}\text { teaching efficacy } \\
\text { (Cronbach's alpha } \\
\text { ranged } .74 \text { to } .77 \text { ) and } \\
\text { personal efficacy ( } \alpha \\
\text { ranged } .64 \text { to } .67 \text { ); } \\
\text { teacher power was } \\
\text { found to have two } \\
\text { dimensions: classroom }\end{array}$ \\
\hline
\end{tabular}




\begin{tabular}{|c|c|c|c|}
\hline & & & $\begin{array}{l}\text { based decision-making } \\
(\alpha=.69) \text { and } \\
\text { school based decision- } \\
\text { making }(\alpha=.80) \text {; } \\
\text { school instructional } \\
\text { climate produced three } \\
\text { dimensions: } \\
\text { positive school } \\
\text { environment ( } \alpha \text { ranged } \\
.74 \text { to } .88) \text {, } \\
\text { lack of impediments to } \\
\text { effective instruction ( } \alpha \\
\text { ranged } .45 \text { to } .59), \\
\text { and collegiality ( } \alpha \\
\text { ranged } .64 \text { to } .71\end{array}$ \\
\hline $\begin{array}{l}\text { Attitudes and } \\
\text { Beliefs on } \\
\text { Classroom Control } \\
\text { Inventory }\end{array}$ & $\begin{array}{l}\text { Martin, Yin \& } \\
\text { Baldwin, } 1998\end{array}$ & $N=281$ & $\begin{array}{l}\text { Cronbach's alpha for } \\
\text { subscales ranged from } \\
.82 \text { to .69) }\end{array}$ \\
\hline $\begin{array}{l}\text { Teacher } \\
\text { Questionnaire }\end{array}$ & $\begin{array}{l}\text { Hoover-Dempsey, } \\
\text { Bassler \& Brissie, } \\
1992\end{array}$ & $N=30$ & $\begin{array}{l}\text { Cronbach's alpha: } \\
\text { teacher efficacy, } .81 \\
\text { teacher perception of } \\
\text { parent efficacy scale, } \\
.79)\end{array}$ \\
\hline $\begin{array}{l}\text { Michigan } \\
\text { Organizational } \\
\text { Assessment } \\
\text { Questionnaire }\end{array}$ & $\begin{array}{l}\text { Seashore, Lawler, } \\
\text { Mirvis \& } \\
\text { Cammann, } 1982\end{array}$ & $N=466$ & Cronbach's alpha $=.77$ \\
\hline
\end{tabular}




\section{APPENDIX G}

Collective Efficacy Research Instruments

\begin{tabular}{|c|c|c|c|}
\hline Instrument/Scale & Designers/Authors & $N$ & $\begin{array}{c}\text { Reliability } \\
\text { Scale/Subscales }\end{array}$ \\
\hline $\begin{array}{l}\text { Collective Efficacy } \\
\text { Scale }\end{array}$ & $\begin{array}{c}\text { Riggs \& Knight, } \\
1992\end{array}$ & & Cronbach's alpha $>.70$ \\
\hline $\begin{array}{l}\text { Collective Teacher } \\
\text { Efficacy Scale }\end{array}$ & Goddard, 2002a & $N=332$ & Cronbach's alpha .94 \\
\hline $\begin{array}{l}\text { Sense of } \\
\text { Powerlessness Scale }\end{array}$ & $\begin{array}{c}\text { Zielinski \& Hoy, } \\
1983\end{array}$ & & Cronbach's alpha .83 \\
\hline $\begin{array}{l}\text { Collective Teacher } \\
\text { Self-Efficacy Scale }\end{array}$ & $\begin{array}{c}\text { Schwarzer, } \\
\text { Schmitz \& } \\
\text { Daytner, } 1999\end{array}$ & $N=300$ & Cronbach's alpha .91 \\
\hline $\begin{array}{l}\text { The Teacher } \\
\text { Efficacy Scale }\end{array}$ & $\begin{array}{c}\text { Gibson \& Dembo, } \\
1984\end{array}$ & & $\begin{array}{l}\text { Cronbach's alpha for } \\
\text { subscales: } \\
\text { personal teaching } \\
\text { efficacy, } .75 \\
\text { general teaching } \\
\text { efficacy, } .79\end{array}$ \\
\hline $\begin{array}{l}\text { Teacher Job } \\
\text { Satisfaction } \\
\text { Questionnaire }\end{array}$ & Lester, 1987 & $N=620$ & Cronbach's alpha $=.93$ \\
\hline $\begin{array}{l}\text { Collective Teacher } \\
\text { Efficacy Scale } \\
\text { (CTES) }\end{array}$ & $\begin{array}{l}\text { Goddard, Hoy \& } \\
\text { Hoy, } 2000\end{array}$ & $N=46$ & Cronbach's Alpha .93 \\
\hline $\begin{array}{l}\text { Teacher Trust in } \\
\text { Colleagues }\end{array}$ & $\begin{array}{c}\text { Hoy and } \\
\text { Kupersmith, } 1985\end{array}$ & $N=452$ & Cronbach's Alpha .93 \\
\hline $\begin{array}{l}\text { Collective Teacher } \\
\text { Efficacy Scale, }\end{array}$ & $\begin{array}{c}\text { Goddard \& } \\
\text { Goddard, } 2001\end{array}$ & $N=438$ & Cronbach's Alpha .96 \\
\hline $\begin{array}{l}\text { Collective Efficacy } \\
\text { Scale }\end{array}$ & Mackenzie, 2001 & $N=384$ & $\begin{array}{l}\text { Cronbach's alpha for } \\
\text { subscales: } \\
\text { teacher efficacy, } .60 \\
\text { collective efficacy, } .64 \text {; } \\
\text { total efficacy, } .72\end{array}$ \\
\hline Teacher Efficacy & Gibson \& Dembo, & & Cronbach's alpha for \\
\hline
\end{tabular}




\begin{tabular}{l|c|c}
\hline Scale & 1984 & $\begin{array}{c}\text { subscales: } \\
\text { general teaching } \\
\text { efficacy, } .77, .58, \text { and } .66 \\
\text { on three test } \\
\text { administration; } \\
\end{array}$ \\
& $\begin{array}{c}\text { personal teaching } \\
\text { efficacy, .50, .82, and .72 } \\
\text { respectively on three } \\
\text { administrations }\end{array}$ \\
\hline
\end{tabular}




\section{APPENDIX H}

Teacher Satisfaction Research Instruments

\begin{tabular}{|c|c|c|c|}
\hline Instrument/Scale & Designers/Authors & $N$ & $\begin{array}{l}\text { Reliability } \\
\text { Scale/Subscales }\end{array}$ \\
\hline $\begin{array}{l}\text { Schools and } \\
\text { Staffing Survey, } \\
\text { (NCES) }\end{array}$ & $\begin{array}{l}\text { National Center for } \\
\text { Education Statistics, } \\
\text { 1999-2000; }\end{array}$ & $N=52,000$ & Cronbach's alpha $=.77$ \\
\hline $\begin{array}{l}\text { Teacher Job } \\
\text { Satisfaction } \\
\text { Questionnaire } \\
\text { (TJSQ) }\end{array}$ & Lester, 1987 & $N=620$ & Cronbach's alpha $=.93$ \\
\hline $\begin{array}{l}\text { Job Diagnostic } \\
\text { Survey }\end{array}$ & $\begin{array}{l}\text { Hackman \& } \\
\text { Oldham, } 1974\end{array}$ & $N=6930$ & Cronbach's alpha $=.77$ \\
\hline $\begin{array}{l}\text { Job Description } \\
\text { Index }\end{array}$ & $\begin{array}{l}\text { Balzer et al., } \\
1997\end{array}$ & & \\
\hline $\begin{array}{l}\text { Job Satisfaction } \\
\text { Scale }\end{array}$ & $\begin{array}{l}\text { Brayfield \& } \\
\text { Rothe, } 1951\end{array}$ & $N=231$ & Cronbach's alpha $=.97$ \\
\hline $\begin{array}{l}\text { General Perceived } \\
\text { Self-Efficacy Scale }\end{array}$ & $\begin{array}{l}\text { Jerusalem and } \\
\text { Schwarzer, } 2001\end{array}$ & & $\begin{array}{l}\text { Cronbach's alphas } \\
\text { ranged } \\
\text { from } .76 \text { to } .90\end{array}$ \\
\hline $\begin{array}{l}\text { Teachers' Sense of } \\
\text { Efficacy Scale-Short }\end{array}$ & $\begin{array}{l}\text { Tschanen-Moran } \\
\text { and Woolfolk- } \\
\text { Hoy, 2001b }\end{array}$ & $\begin{array}{l}3 \text { studies } \\
N=224 \\
N=217 \\
N=183\end{array}$ & $\begin{array}{l}\text { Cronbach's alphas for } \\
\text { total scale, } .90 ; \\
\text { engagement, } .81 ; \\
\text { instruction, } .86 ; \\
\text { management, } .86\end{array}$ \\
\hline $\begin{array}{l}\text { Teacher Motivation } \\
\text { and Job Satisfaction } \\
\text { Survey }\end{array}$ & Mertler, 2001 & $\begin{array}{l}N=969 \\
N=2202\end{array}$ & $\begin{array}{l}\text { Cronbach's alphas } \\
\text { were - } \\
\text { teaching competence, } \\
.56 \\
\text { administration control, } \\
.73 ; \\
\text { organizational culture, } \\
.78 \text { and } \\
\text { teacher satisfaction, } \\
.77\end{array}$ \\
\hline
\end{tabular}




\begin{tabular}{|c|c|c|c|}
\hline $\begin{array}{l}\text { Teacher Job } \\
\text { Satisfaction Survey }\end{array}$ & $\begin{array}{l}\text { National } \\
\text { Database of } \\
\text { Preservice } \\
\text { Teachers, 1988- } \\
1992\end{array}$ & $N=2054$ & \\
\hline $\begin{array}{l}\text { Teacher Satisfaction } \\
\text { Survey }\end{array}$ & $\begin{array}{l}\text { Kappa Delta Pi, } \\
1990\end{array}$ & $N=3000$ & \\
\hline $\begin{array}{l}\text { Job Descriptive } \\
\text { Index (JDI) }\end{array}$ & $\begin{array}{l}\text { Bowling Green } \\
\text { State University, } \\
1985,1997,\end{array}$ & $N=682$ & $\begin{array}{l}\text { Cronbach's alphas: } \\
\text { work, .84; } \\
\text { pay, .80; } \\
\text { promotions, .86; } \\
\text { supervision, .87; } \\
\text { coworkers, } .88\end{array}$ \\
\hline
\end{tabular}




\section{APPENDIX I.}

Intent to Turnover Research Instruments

\begin{tabular}{|c|c|c|c|}
\hline Instrument/Scale & Designers/Authors & $N$ & $\begin{array}{l}\text { Reliability } \\
\text { Scale/Subscales }\end{array}$ \\
\hline $\begin{array}{l}\text { Michigan } \\
\text { Organizational } \\
\text { Assessment } \\
\text { Questionnaire } \\
\text { (MOAQ }\end{array}$ & $\begin{array}{l}\text { Cammann, } \\
\text { Fichman, Jenkins, } \\
\text { \& Klesh, } 1982\end{array}$ & $N=466$ & $\begin{array}{l}\text { Cronbach's alpha }=.90 \\
\text { Intent to Turnover }, .83\end{array}$ \\
\hline $\begin{array}{l}\text { Organizational } \\
\text { Commitment } \\
\text { Questionnaire }\end{array}$ & $\begin{array}{l}\text { Meyer \& Allen, } \\
1997\end{array}$ & & Cronbach's alpha $=.90$ \\
\hline $\begin{array}{l}\text { Organizational } \\
\text { Commitment } \\
\text { Questionnaire }\end{array}$ & $\begin{array}{l}\text { Mowday, Steers } \\
\& \text { Porter, } 1979\end{array}$ & $N=2563$ & $\begin{array}{l}\text { Cronbach's alpha } \\
\text { coefficients ranged from } \\
.82 \text { to } .93\end{array}$ \\
\hline $\begin{array}{l}\text { Minnesota } \\
\text { Satisfaction } \\
\text { Questionnaire }\end{array}$ & Weiss et al., 1967 & $N=1793$ & $\begin{array}{l}\text { Cronbach's alpha } \\
\text { coefficients ranged from } \\
.93 \text { to } .78\end{array}$ \\
\hline $\begin{array}{l}\text { Schools and } \\
\text { Staffing Survey } \\
\text { (SASS; Teacher } \\
\text { Follow-up Survey } \\
\text { (TFS) }\end{array}$ & $\begin{array}{l}\text { U.S. Department } \\
\text { of Education's } \\
\text { National Center } \\
\text { for Education } \\
\text { Statistics }\end{array}$ & $N=50,000$ & \\
\hline $\begin{array}{l}\text { Staff Climate } \\
\text { Survey, a subset of } \\
\text { the Teacher Survey }\end{array}$ & $\begin{array}{l}\text { Center for the } \\
\text { Study of Teaching } \\
\text { and Policy (CTP }\end{array}$ & $N=3,200$ & \\
\hline $\begin{array}{l}\text { Teacher Service } \\
\text { Record/Certification } \\
2000-2001 \text { and } \\
\text { Teacher Service } \\
\text { Record/ } \\
\text { Certification } 2001 \text { - } \\
2002 \text { ) }\end{array}$ & $\begin{array}{l}\text { Chicago Acorn, } \\
2003\end{array}$ & $N=2,377$ & \\
\hline $\begin{array}{l}\text { Intent to Leave } \\
\text { Survey }\end{array}$ & $\begin{array}{l}\text { Johnsrud \& } \\
\text { Rosser, } 1997\end{array}$ & $N=1,293$ & $\begin{array}{l}\text { Cronbach's alpha } \\
\text { coefficients - career } \\
\text { support, .85; } \\
\text { working conditions, .73; } \\
\text { perceptions of } \\
\text { discrimination, .79; } \\
\text { program review and }\end{array}$ \\
\hline
\end{tabular}




\begin{tabular}{l|l|l|l}
\hline & & $\begin{array}{l}\text { government intervention, } \\
71 ; \text { and }\end{array}$ \\
Measure of & Price \& Mueller, \\
Turnover Intent & $N 986$ & $\begin{array}{l}\text { issues of diversity, } .83 \\
\text { Cronbach's alpha } \\
\text { coefficient for turnover } \\
\text { intent ranged from .85 to } \\
90\end{array}$ \\
\hline
\end{tabular}




\section{APPENDIX J}

\section{Turnover Cost Models}

\begin{tabular}{|c|c|}
\hline Model Type & Model Description \\
\hline Model One & $\begin{array}{l}\text { This model by Advantage Assessment Inc. uses the number of } \\
\text { leavers, the annual salary for these leavers, information on hiring } \\
\text { including the number of applicants for each job opening and the } \\
\text { number of employees interviewed per job opening. }\end{array}$ \\
\hline Model Two & $\begin{array}{l}\text { The Sorenson (1995) and Jones (1999) model includes three primary } \\
\text { categories: (1) hiring costs, (2) training costs, and (3) lost } \\
\text { productivity costs. Hiring costs include advertising, the time and } \\
\text { effort for reading applications, scheduling and conducting } \\
\text { interviews, and post-employment hiring tasks. Training costs } \\
\text { involve both orientation activities and training sessions. Productivity } \\
\text { loss calculations usually include decline in productivity due to } \\
\text { differences in performance between the veteran leaver and the } \\
\text { trainee - often expressed in terms of student performance. }\end{array}$ \\
\hline Model Three & $\begin{array}{l}\text { The People Sense model incorporates the three categories in Model } \\
\text { Two and introduces vacancy costs as a contributor to the cost of } \\
\text { turnover. This model requires the amount for the leaver's annual } \\
\text { salary and benefits. Benefits are estimated at twenty five percent of } \\
\text { the leaver's annual pay. }\end{array}$ \\
\hline Model Four & $\begin{array}{l}\text { Cascio's model of turnover costs specifies four types of cost } \\
\text { involved in turnover: ( } 1 \text { ) separation costs, (2) replacement hiring } \\
\text { costs, ( } 3 \text { ) training costs, and (4) learning curve loss (Cascico, 1987). } \\
\text { Separation costs (exit interviews, cost of interviewer's time for } \\
\text { preparation and interviewing and the cost for the leaver's time for } \\
\text { interview. Hiring costs (communication of the availability for the } \\
\text { position, pre-employment administrative tasks, and pre-employment } \\
\text { testing costs. Training costs (costs of training time for the trainer } \\
\text { and trainee during formal training. Learning curve loss ( } 1 \text {. divide the } \\
\text { leaver's annual salary by the midpoint of pay grade and multiply this } \\
\text { by } 100, \text {, } 2 \text {. divide the new employee's salary by the midpoint and } \\
\text { multiply by } 100 \text {, and ( } 3 \text {. subtract the new employee's ration from the } \\
\text { leaver's ratio. }\end{array}$ \\
\hline Model Five & $\begin{array}{l}\text { This comprehensive model asserts that turnover costs must include } \\
\text { termination or separation costs, hiring costs, vacancy costs, learning } \\
\text { curve loss, and training costs. These categories are described in } \\
\text { much the same way as these categories in previous models (3-6). }\end{array}$ \\
\hline
\end{tabular}




\section{APPENDIX K}

Letter to Institutional Review Board for Expedited Review

February 2, 2007

Human Subjects Protection Program Office

University of Louisville

501 E. Broadway, STE 200

Louisville, KY 40202

Dear Members of the Institutional Review Board:

This letter is a request for an expedited review for a research study. The purpose of this study is to investigate the relationships between school climate, school culture, teacher efficacy and collective efficacy and job satisfaction, and intent to turnover in select Kentucky school districts operating on YRE calendar reform.

Upon approval, the present study will commence in the Spring of 2007. The subjects in this study will consist of teachers employed in school districts in Kentucky that operate on year-round education school calendars.

The principal investigator in this study is Dr. Thomas G. Reio, Jr. This is a doctoral dissertation research study, being conducted by Joseph W. Mattingly in the Department of Leadership, Foundations and Human Resource Education.

I have enclosed the required documents for your review and approval. Your prompt response will be greatly appreciated.

Regards,

Dr. Thomas G. Reio, Jr.

Dr. Thomas G. Reio, Jr.

Leadership, Foundations, and Human Resource Education

College of Education and Human Development

University of Louisville

Louisville, KY 40292

(502) 852-0639
Joseph W. (J.W.) Mattingly

Joseph W. (J.W.) Mattingly

Doctoral Student, University of Louisville

439 Bloomfield Road

Bardstown, KY 40004

(502) $331-8800$ 


\section{APPENDIX L}

Data Collection Protocol Summary Table

\begin{tabular}{l|l}
\hline Procedural Steps & Time Period \\
\hline E-mail Notices to Participants & Day Schedule \\
\hline 1. Sent Pre-Notice E-mail to Teachers & Day 1 - Tuesday \\
2. Sent Survey E-mail to Teachers & Day 3 - Thursday \\
3. Sent Reminder E-mail to Teachers Not Completing \\
Questionnaire & Day 7 - Tuesday \\
\hline Data Management & \\
\hline 4. Analyzed Questionnaire Download Database for & Completed Questionnaire Data and Return Rate \% \\
5. Sent Thank-You/ Reminder E-mail to Teachers & Day 8- Wednesday \\
6. Sent Thank-You/ Reminder E-mail to Principals & Day 9-Thursday \\
7. Sent Thank-You/ Reminder E-mail to Superintendent & Day 9-Thursday \\
8. Loaded Questionnaire Data in SPSS Statistical Program & Day 10 - Friday \\
9. Analyzed Statistical Results & Day 11-Continuing
\end{tabular}




\section{Teacher Job Satisfaction Scale}

Directions: The part of the questionnaire is designed to help us understand how you think about the following important matters related to your teaching job satisfaction. Please indicate your opinion about each of the teacher job satisfaction statement ratings below ranging from (I) "Strongly Agree" to (5) "Strongly Disagree" by clicking/checking the appropriate circle under the rating indicator you select. Your answers are confidential. Your answers are confidential.

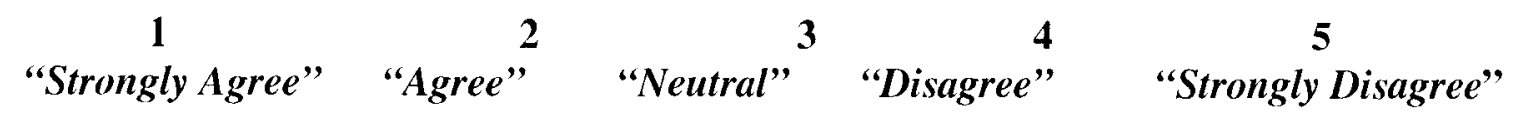

1. I am satisfied with the support I receive at my school.

2. I am satisfied with my learning experiences at school.

3. Generally, I have had a positive learning experience in this school.

4. I am satisfied with the feedback I have received about my performance at my job.

5. All in all, I am satisfied with my job as a teacher.

6. In general, I don't like my job as a teacher.

7. In general, I like working at my school.

8. The benefit package we have is equitable.

9. I do not like the climate where I work.

10. I am satisfied with my pay.

11. I am not satisfied with the promotional opportunities where I work.

12. I am satisfied with the way I am treated by the people with whom I work.

13. I have the opportunity to develop my skills and abilities in this job.

14. All in all, I am satisfied with teaching in the year-round education calendar.

15. In general, I am satisfied with student achievement in the year-round calendar.

16. All in all, I am satisfied with instructional flexibility in the year-round calendar.

17. Overall, I am satisfied with my teaching effectiveness in the year-round calendar.

\begin{tabular}{|c|c|c|c|c|}
\hline 1 & 2 & 3 & 4 & 5 \\
\hline $\mathrm{O}$ & $\mathrm{O}$ & $\mathrm{O}$ & $\mathrm{O}$ & $\mathrm{O}$ \\
\hline $\mathrm{I}$ & 2 & 3 & 4 & 5 \\
\hline $\mathrm{O}$ & $\mathrm{O}$ & $\mathrm{O}$ & $\mathrm{O}$ & $\mathrm{O}$ \\
\hline 1 & 2 & 3 & 4 & 5 \\
\hline $\mathrm{O}$ & $\mathrm{O}$ & $\mathrm{O}$ & $\mathrm{O}$ & $\mathrm{O}$ \\
\hline $\mathrm{1}$ & 2 & 3 & 4 & 5 \\
\hline $\mathrm{O}$ & $\mathrm{O}$ & $\mathrm{O}$ & $\mathrm{O}$ & $\mathrm{O}$ \\
\hline $\mathrm{I}$ & 2 & 3 & 4 & 5 \\
\hline $\mathrm{O}$ & $\mathrm{O}$ & $\mathrm{O}$ & $\mathrm{O}$ & $\mathrm{O}$ \\
\hline $\mathrm{I}$ & 2 & 3 & 4 & 5 \\
\hline $\mathrm{O}$ & $\mathrm{O}$ & $\mathrm{O}$ & $\mathrm{O}$ & $\mathrm{O}$ \\
\hline $\mathrm{I}$ & 2 & 3 & 4 & 5 \\
\hline $\mathrm{O}$ & $\mathrm{O}$ & $\mathrm{O}$ & $\mathrm{O}$ & $\mathrm{O}$ \\
\hline $\mathrm{I}$ & 2 & 3 & 4 & 5 \\
\hline $\mathrm{O}$ & $\mathrm{O}$ & $\mathrm{O}$ & $\mathrm{O}$ & $\mathrm{O}$ \\
\hline $\mathrm{I}$ & 2 & 3 & 4 & 5 \\
\hline $\mathrm{O}$ & $\mathrm{O}$ & $\mathrm{O}$ & $\mathrm{O}$ & $\mathrm{O}$ \\
\hline $\mathrm{I}$ & 2 & 3 & 4 & 5 \\
\hline $\mathrm{O}$ & $\mathrm{O}$ & $\mathrm{O}$ & $\mathrm{O}$ & $\mathrm{O}$ \\
\hline $\mathrm{I}$ & 2 & 3 & 4 & 5 \\
\hline $\mathrm{O}$ & $\mathrm{O}$ & $\mathrm{O}$ & $\mathrm{O}$ & $\mathrm{O}$ \\
\hline $\mathrm{I}$ & 2 & 3 & 4 & 5 \\
\hline $\mathrm{O}$ & $\mathrm{O}$ & $\mathrm{O}$ & $\mathrm{O}$ & $\mathrm{O}$ \\
\hline $\mathrm{I}$ & 2 & 3 & 4 & 5 \\
\hline $\mathrm{O}$ & $\mathrm{O}$ & $\mathrm{O}$ & $\mathrm{O}$ & $\mathrm{O}$ \\
\hline 1 & 2 & 3 & 4 & 5 \\
\hline $\mathrm{O}$ & $\mathrm{O}$ & $\mathrm{O}$ & $\mathrm{O}$ & $\mathrm{O}$ \\
\hline $\mathrm{I}$ & 2 & 3 & 4 & 5 \\
\hline $\mathrm{O}$ & $\mathrm{O}$ & $\mathrm{O}$ & $\mathrm{O}$ & $\mathrm{O}$ \\
\hline $\mathrm{I}$ & 2 & 3 & 4 & 5 \\
\hline $\mathrm{O}$ & $\mathrm{O}$ & $\mathrm{O}$ & $\mathrm{O}$ & $\mathrm{O}$ \\
\hline 1 & 2 & 3 & 4 & 5 \\
\hline $\mathrm{O}$ & $\mathrm{O}$ & $\mathrm{O}$ & $\mathrm{O}$ & $\mathrm{O}$ \\
\hline
\end{tabular}




\section{Teacher Intent to Turnover Scale}

Directions: Think about the following important matters related to your teaching job.

Please indicate your opinion about each of the intent to turnover statement ratings below ranging from (1) "Not At All Likely" to (7) "Extremely Likely" by clicking/checking the appropriate circle under the rating indicator you select. Your answers are confidential.

\begin{tabular}{|c|c|c|c|c|c|c|c|c|}
\hline $\begin{array}{c}1 \\
\text { "Not At All Likely" }\end{array}$ & $\begin{array}{ccc}3 & 4 & 5 \\
\text { "Somewhat Likely" } & \text { "Quite I }\end{array}$ & $\stackrel{5}{5}$ "Quite Likely" & \multicolumn{6}{|c|}{$\begin{array}{lc}6 & 7 \\
\text { "Extremely Likely" }\end{array}$} \\
\hline \multirow{2}{*}{\multicolumn{2}{|c|}{$\begin{array}{l}\text { 1. How likely is it that you could find a job with another } \\
\text { employer with about the same pay and benefits you } \\
\text { have now? }\end{array}$}} & 1 & 2 & 3 & 4 & 5 & 6 & 7 \\
\hline & & O & O & 0 & $\mathrm{O}$ & $\mathrm{O}$ & $\mathrm{O}$ & $\mathrm{O}$ \\
\hline \multirow{2}{*}{\multicolumn{2}{|c|}{$\begin{array}{l}\text { 2. How likely is it that you will actively look for a new } \\
\text { job in the next year? }\end{array}$}} & 1 & 2 & 3 & 4 & 5 & 6 & 7 \\
\hline & & $\mathrm{O}$ & $O$ & 0 & $\mathrm{O}$ & $\mathrm{O}$ & $\mathrm{O}$ & $\mathrm{O}$ \\
\hline \multirow{2}{*}{\multicolumn{2}{|c|}{ 3. I often think about quitting. }} & 1 & 2 & 3 & 4 & 5 & 6 & 7 \\
\hline & & $\mathrm{O}$ & O & $\mathrm{O}$ & O & O & O & $\mathrm{O}$ \\
\hline \multirow{2}{*}{\multicolumn{2}{|c|}{$\begin{array}{l}\text { 4. If I had the chance, I would take a similar job with a } \\
\text { traditional calendar school district. }\end{array}$}} & 1 & 2 & 3 & 4 & 5 & 6 & 7 \\
\hline & & O & $\mathrm{O}$ & $O$ & O & O & $\mathrm{O}$ & $\mathrm{O}$ \\
\hline
\end{tabular}

\section{GENERAL COMMENTS}

For me, personally and professionally, the year-round education calendar has ..... 


\section{APPENIDIX R}

Reminder E-mail to Superintendents

(sent to call Superintendents)

Dear Superintendent XXX,

This is a reminder regarding a doctoral research study to investigate the relationships between school climate, school culture, teacher efficacy and collective efficacy and job satisfaction, and intent to turnover in select Kentucky school districts operating on YRE calendar reform. Please remind your principals and teachers, as well as encourage those teachers who have not yet filled out the survey to please do so.

Your assistance has been greatly appreciated.

Regards,

Dr. Thomas (i. Reio, Jr.

Dr. Thomas G. Reio, Jr.

Leadership, Foundations, and Human Resource Education

College of Education and Human Development

University of Louisville

Louisville, KY 40292

(502) 852-(06.39
Joseph W. (J.W.) Mattingly

Joseph W. (J.W.) Mattingly Doctoral Student,

University of Louisville 439 Bloomfield Road Bardstown, KY 400)4

(502) 331-8800 


\author{
APPENDIX S \\ Reminder E-mail to Principals \\ (sent to all Principals)
}

Dear Principal XXX,

This is a reminder regarding a doctoral research study to investigate the relationships between school climate, school culture, teacher efficacy and collective eflicacy and job satisfaction, and intent to turnover in select Kentucky school districts operating on YRE calendar reform. In order for this research study to be a success. we will need a high response rate. Please remind your teachers who have not yet filled out the questionnaire to please do so. The data is important to the study and your school.

Your assistance has been greatly appreciated.

Regards,

Dr. Thomas G. Reio, Jr.

Joseph W. (J.W.) Mattingly

Dr. Thomas G. Reio, Jr.

Joseph W. (J.W.) Mattingly

Leadership, Foundations, and Human Resource Education

Doctoral Student,

College of Education and Human Development

Universily of Louisville

University of Louisville

439 Bloomfield Road

Louisville, KY 40292

Bardstown, KY 40004

(502) 852-(0639

(502) 331-8800 


\author{
APPENDIX T \\ Reminder E-mail to Teacher Participants \\ (sent to all school)
}

Dear Fellow Colleagues,

For those of you who have not yet completed the Web-based questionnaire regarding the relationships between school climate, school culture, teacher efficacy and collective efficacy and job satisfaction, and intent to turnover in select Kentucky school districts operating on YRE calendar reform, the Weblink will remain active until March 10, 2007. You can access the survey by clicking on the following link:

\title{
http://bardstownschools.us/Research/default.htm .
}

In order for this study to be a success, a high response rate is needed. Your responses are very valuable to this study and greatly appreciated.

I would like to thank each of you who have already completed the Web questionnaire. Your contribution to this study will help other teachers, such as yourself, as well as schools/school districts considering alternative school calendars.

Regards,

Dr. Thomas G. Reio, Jr.

Dr. Thomas G. Reio, Jr.

Leadership. Foundations, and Human Resource Education College of Education and Human Development

University of Louisville

Louisville, KY 40292

(502) $852-0639$
Joseph W. (J.W.) Mattingly

Joseph W. (J.W.) Mattingly

Doctoral Student.

University of Louisville

439 Bloomfield Road

Bardstown, KY 40004

(502) 331-8800 


\section{APPENDIX U \\ Reminder/Thank You E-mail to Superintendents}

Dear Superintendent XXX,

This is the final e-mail regarding a doctoral research study to investigate the relationships between school climate, school culture, teacher efficacy and collective efficacy and job satisfaction, and intent to turnover in select Kentucky school districts operating on YRE calendar reform. In order for this research study to be a success, we will need between 50-60\% response rate. Therefore, I am asking that you please remind your principals and teachers, as well as encourage those who have not yet lilled out the web-based questionnaire survey to please do so.

Your assistance has been greatly appreciated.

Regards.

Dr. Thomas G. Reio, Jr.

Joseph W. (J.W.) Mattingly

Dr. Thomas G. Reio, J1.

Leadership, Foundations, and Human Resource Education

Joseph W. (J.W.) Mattingly

College of Education and Human Development

Doctoral Student,

University of Louisville

University of Louisville

Louisville, KY 40292

439 Bloomfield Road

(502) 852-0639

Bardstown, KY 40004

(502) $331-8800$ 


\section{APPENDIX V \\ Reminder/Thank You E-mail to Principals}

Dear Principal XXX,

This is the final e-mail regarding a doctoral research study to investigate the relationships between school climate, school culture, teacher efficacy and collective efficacy and job satisfaction, and intent to turnover in select Kentucky school districts operating on YRE calendar reform. In order for this research study to be a success, we will need between $50-60 \%$ response rate. Therefore, I am asking that you please remind your teachers, as well as encourage those who have not yet filled out the web-based questionnaire to please do so.

Your assistance has been greatly appreciated.

Regards,

Dr. Thomas G. Reio, Jr.

Joseph W. (J.W.) Mattingly

Dr. Thomas G. Reio. Jr.

Joseph W. (J.W.) Mattingly

Leadership. Foundations, and Human Resource Education Doctoral Student,

College of Education and Human Development University of Louisville

University of Louisville 439 Bloomfield Road

Louisville, KY 40292

Bardstown, KY 40004

(502) 852-0639

(502) $331-8800$ 


\section{APPENDIX W}

Reminder/Thank You E-mail to Teacher Participants

Dear Fellow Colleagues,

For those of you who have not yet completed the Web-based survey to investigate the relationships between school climate, school culture, teacher efficacy and collective efficacy and job satisfaction, and intent to turnover in select Kentucky school districts operating on YRE calendar reform, the Weblink will remain active until the March 2nd. You can access the web-survey by clicking on the following link:

\section{http://bardstownschools.us/Research/Research_survey.asp}

In order for this study to be a success, 50-60\% response rate is needed. Therefore, I am asking that you please take time to complete the survey, which will take about 20 minutes. Your responses are very valuable to the success of this study and greatly appreciated.

I would like to thank each of you who have already completed the Web survey. Your contribution to this study will help other teachers, such as yourself, as well as schools/school districts considering alternative school calendars.

Regards,

Dr. Thomas G. Reio, Jr.

Dr. Thomas G. Reio, Jr.

Leadership, Foundations, and Human Resource Education

College of Education and Human Development

University of Louisville

Louisville, KY 40292

(502) 852-0639
Joseph W. (J.W.) Mattingly

Joseph W. (J.W.) Mattingly

Doctoral Student.

University of Louisville

439 Bloomfield Road

Bardstown, KY 40004

(502) 331-8800 
APPENDIX X

Summary of Demographic Information

Demographic Categories for Total Districts and Individual Districts

\begin{tabular}{|c|c|c|c|c|c|c|c|c|c|c|}
\hline & \multicolumn{2}{|c|}{ All Districts } & \multicolumn{2}{|c|}{ District A } & \multicolumn{2}{|c|}{ District B } & \multicolumn{2}{|c|}{ District C } & \multicolumn{2}{|c|}{ District D } \\
\hline & Freq & $\%$ & Freq & $\%$ & Freq & $\%$ & Freq & $\%$ & Freq & $\%$ \\
\hline \multicolumn{11}{|l|}{ Ethnicity } \\
\hline $\begin{array}{l}\text { African- } \\
\text { American }\end{array}$ & 28 & 4 & 5 & 4 & 0 & () & 0 & () & 1 & 1 \\
\hline $\begin{array}{l}\text { Asian- } \\
\text { American }\end{array}$ & 2 & () & 2 & 2 & 0 & () & 0 & () & () & () \\
\hline Caucasian & 709 & 95 & 128 & 94 & 24 & 100 & 162 & 99 & 122 & 98 \\
\hline $\begin{array}{l}\text { Hispanic- } \\
\text { American }\end{array}$ & 2 & () & 0 & 0 & 0 & () & () & 0 & 1 & 1 \\
\hline Other & 3 & () & () & 0 & () & () & 2 & 1 & 0 & 0 \\
\hline Total & 744 & 100 & 136 & 100 & 24 & 100 & 164 & 100 & 122 & 100 \\
\hline \multicolumn{11}{|l|}{ Age } \\
\hline $21-29$ & 146 & 20 & 21 & 15 & 6 & 25 & 44 & 27 & 16 & 13 \\
\hline $30-39$ & 222 & 30 & 57 & 42 & 6 & 25 & 43 & 26 & 36 & 29 \\
\hline 4()$-49$ & 200 & 27 & 31 & 23 & 4 & 17 & 39 & 24 & 42 & 34 \\
\hline $50-59$ & 160 & 21 & 23 & 17 & 8 & 33 & 39 & 24 & 26 & 21 \\
\hline $60 \&$ Greater & 18 & 2 & 3 & 2 & 0 & 0 & 1 & 1 & 4 & 3 \\
\hline Total & 746 & 100 & 135 & 100 & 24 & 100 & 166 & 100 & 124 & 100 \\
\hline \multicolumn{11}{|l|}{ Gender } \\
\hline Female & 592 & 80 & 105 & 77 & 21 & 88 & 128 & 77 & 91 & 73 \\
\hline Male & 149 & 20 & 28 & 21 & 3 & 13 & 38 & 23 & 31 & 25 \\
\hline Total & 741 & 100 & 133 & 100 & 24 & 100 & 166 & 100 & 122 & 98 \\
\hline
\end{tabular}




\begin{tabular}{|c|c|c|c|c|c|c|c|c|c|c|}
\hline & \multicolumn{2}{|c|}{ All Districts } & \multicolumn{2}{|c|}{ District A } & \multicolumn{2}{|c|}{ District B } & \multicolumn{2}{|c|}{ District $\mathrm{C}$} & \multicolumn{2}{|c|}{ District D } \\
\hline & Freq & $\%$ & Freq & $\%$ & Freq & $\%$ & Freq & $\%$ & Freq & $\%$ \\
\hline \multicolumn{11}{|c|}{ Highest Degree } \\
\hline $\begin{array}{l}\text { Bachelor's } \\
\text { Degree }\end{array}$ & 137 & 18 & 23 & 17 & 4 & 17 & 29 & 17.5 & 20 & 16 \\
\hline $\begin{array}{l}\text { Master's } \\
\text { Degree }\end{array}$ & 364 & 49 & 69 & 51 & 11 & 46 & 91 & 55 & 56 & 45 \\
\hline $\begin{array}{l}\text { Rank I } \\
\text { Degree }\end{array}$ & 236 & 31 & 42 & 31 & 9 & 38 & 44 & 27 & 48 & 39 \\
\hline $\begin{array}{l}\text { Doctoral } \\
\text { Degree }\end{array}$ & 6 & 1 & () & () & 0 & () & 1 & .6 & () & () \\
\hline Total & 743 & 100 & 1.34 & 100 & 24 & 100 & 165 & 99 & 124 & 100 \\
\hline \multicolumn{11}{|c|}{ School Level Placement } \\
\hline $\begin{array}{l}\text { Preschool } \\
\text { Education }\end{array}$ & 9 & 1.2 & 5 & 4 & () & 0 & () & 0 & 1 & 1 \\
\hline $\begin{array}{l}\text { Elementary } \\
\text { School }\end{array}$ & 390 & 53 & 66 & 49 & 8 & 33 & 96 & 58 & 57 & 46 \\
\hline $\begin{array}{l}\text { Middle } \\
\text { School }\end{array}$ & 131 & 18 & 25 & 18 & 6 & 25 & 29 & 18 & 25 & 20 \\
\hline High School & 213 & 29 & 39 & 29 & 9 & 38 & 41 & 25 & 41 & 33 \\
\hline Total & 743 & 100 & 135 & 99 & 23 & 96 & 166 & 100 & 124 & 100 \\
\hline \multicolumn{11}{|c|}{ Instructional Content Area } \\
\hline $\begin{array}{l}\text { Self- } \\
\text { Contained } \\
\text { Classroom }\end{array}$ & 227 & 31 & 54 & 40 & 6 & 25 & 57 & 34 & 34 & 27 \\
\hline $\begin{array}{l}\text { Reading } \\
\text { Instruction }\end{array}$ & 54 & 7 & 4 & 2.9 & 3 & 13 & 14 & 8 & 6 & 5 \\
\hline $\begin{array}{l}\text { Language } \\
\text { Arts } \\
\text { Instruction }\end{array}$ & 53 & 7 & 8 & 6 & 2 & 8 & 12 & 7 & 10 & 8 \\
\hline $\begin{array}{l}\text { Math } \\
\text { Instruction }\end{array}$ & 58 & 8 & 8 & 6 & 3 & 13 & 7 & 4 & 10 & 8 \\
\hline $\begin{array}{l}\text { Science } \\
\text { Instruction }\end{array}$ & 72 & 9 & 12 & 9 & 3 & 13 & 18 & 11 & 15 & 12 \\
\hline $\begin{array}{l}\text { Social Studies } \\
\text { Instruction }\end{array}$ & 60 & 8 & 12 & 9 & 2 & 8 & 14 & 8 & 9 & 7 \\
\hline $\begin{array}{l}\text { Arts \& } \\
\text { Humanities } \\
\text { Instruction }\end{array}$ & 29 & 4 & 5 & 4 & 1 & 4 & 10 & 6 & 2 & 2 \\
\hline
\end{tabular}




\begin{tabular}{|c|c|c|c|c|c|c|c|c|c|c|}
\hline & \multicolumn{2}{|c|}{ All Districts } & \multicolumn{2}{|c|}{ District A } & \multicolumn{2}{|c|}{ District B } & \multicolumn{2}{|c|}{ District C } & \multicolumn{2}{|c|}{ District D } \\
\hline & Freq & $\%$ & Freq & $\%$ & Freq & $\%$ & Freq & $\%$ & Freq & $\%$ \\
\hline $\begin{array}{l}\text { Practical } \\
\text { Living } \\
\text { Instruction }\end{array}$ & 37 & 5 & 5 & 4 & 1 & 4 & 8 & 5 & 7 & 6 \\
\hline $\begin{array}{l}\text { Other } \\
\text { Instruction }\end{array}$ & 151 & 20 & 28 & 21 & 3 & 13 & 24 & 15 & 31 & 25 \\
\hline Total & 741 & 100 & 136 & 100 & 24 & 100 & 164 & 99 & 124 & 100 \\
\hline \multicolumn{11}{|c|}{ Years of Teaching Experience } \\
\hline $1-5$ Years & 177 & 25 & 31 & 23 & 5 & 21 & 44 & 27 & 26 & 21 \\
\hline 6-10 Years & 169 & 23 & 46 & 34 & 3 & 13 & 38 & 23 & 19 & 15 \\
\hline $11-15$ Years & 126 & 17 & 16 & 12 & 4 & 17 & 26 & 16 & 23 & 19 \\
\hline $16-20$ Years & 83 & 12 & 13 & 10 & 4 & 17 & 23 & 14 & 17 & 14 \\
\hline $21-25$ Ycars & 65 & 12 & 12 & 9 & 2 & 8 & 12 & 7 & 10 & 8 \\
\hline 26-30) Years & 61 & 9 & 8 & 6 & 4 & 17 & 11 & 7 & 14 & 11 \\
\hline $\begin{array}{l}31 \text { or More } \\
\text { Years }\end{array}$ & 39 & 5 & 5 & 4 & 1 & 4 & 7 & 4 & 13 & 11 \\
\hline Total & 723 & 100 & 131 & 96 & 23 & 96 & 161 & 97 & 124 & 98 \\
\hline \multicolumn{11}{|c|}{ Years of Teaching Experience in a YRE Calendar } \\
\hline $1-3$ Years & 184 & 25 & 19 & 14 & 6 & 25 & 46 & 28 & 29 & 23 \\
\hline 4-6 Years & 163 & 23 & 34 & 25 & 4 & 17 & 39 & 24 & 21 & 17 \\
\hline 7-9 Years & 201 & 28 & 35 & 26 & 6 & 25 & 55 & 33 & 30 & 24 \\
\hline 10-11 Years & 176 & 24 & 43 & 32 & 7 & 29 & 21 & 13 & 42 & 34 \\
\hline Totals & 724 & 100 & 131 & 96 & 23 & 96 & 161 & 97 & 122 & 98 \\
\hline \multicolumn{11}{|c|}{ Salary Range } \\
\hline $\begin{array}{l}\$ 20.001- \\
\$ 30.000\end{array}$ & 31 & 4 & 4 & 3 & 3 & 13 & 1 & .6 & 6 & 5 \\
\hline $\begin{array}{l}\$ 30,001- \\
\$ 40,000\end{array}$ & 232 & 32 & 23 & 17 & 7 & 29 & 61 & 37 & 33 & 27 \\
\hline $\begin{array}{l}\$ 40,(001- \\
\$ 50,(000\end{array}$ & 289 & 39 & 57 & 42 & 10 & 42 & 62 & 37 & 50 & 40 \\
\hline $\begin{array}{l}\$ 50,001- \\
\$ 60,000\end{array}$ & 157 & 21 & 39 & 29 & 4 & 17 & 38 & 23 & 25 & 20 \\
\hline $\begin{array}{l}\$ 60,001- \\
\$ 70,000\end{array}$ & 23 & 3 & 10 & 7 & ) & 0 & 2 & 1 & 5 & 4 \\
\hline $\begin{array}{l}\$ 70,001 \& \\
\text { Higher }\end{array}$ & 5 & 1 & 1 & 1 & 0 & 0 & 1 & 1 & 2 & 2 \\
\hline Total & 737 & 100 & 134 & 99 & 24 & 100 & 165 & 99 & 124 & 97 \\
\hline
\end{tabular}




\begin{tabular}{|c|c|c|c|c|c|c|c|c|c|c|}
\hline & \multicolumn{2}{|c|}{ District $\mathrm{E}$} & \multicolumn{2}{|c|}{ District $F$} & \multicolumn{2}{|c|}{ District $G$} & \multicolumn{2}{|c|}{ District $\mathrm{H}$} & \multicolumn{2}{|c|}{ District I } \\
\hline & Freq & $\%$ & Freq & $\%$ & Freq & $\%$ & Freq & $\%$ & Freq & $\%$ \\
\hline \multicolumn{11}{|l|}{ Ethnicity } \\
\hline $\begin{array}{l}\text { African- } \\
\text { American }\end{array}$ & 0 & 0 & 1 & 2 & 1 & 3 & 18 & 11 & 2 & 6 \\
\hline $\begin{array}{l}\text { Asian- } \\
\text { American }\end{array}$ & () & 0 & () & () & 0 & 0 & 0 & 0 & () & () \\
\hline Catuasian & 18 & 100 & 41 & 98 & 30 & 91 & 147 & 89 & 32 & 94 \\
\hline $\begin{array}{l}\text { Hispanic- } \\
\text { American }\end{array}$ & 0 & 0 & () & () & 1 & 3 & () & () & () & () \\
\hline Other & () & () & () & () & 1 & 3 & () & () & () & () \\
\hline Total & 18 & 100 & 43 & 100 & 33 & 100 & 165 & 100 & 34 & 100 \\
\hline \multicolumn{11}{|l|}{ Age } \\
\hline $21-29$ & 10 & 56 & 13 & 30 & 5 & 15 & 23 & 14 & 7 & 21 \\
\hline 30$)-39$ & 5 & 28 & 11 & 26 & 8 & 24 & 43 & 26 & 11 & 32 \\
\hline 4()$-49$ & 2 & 11 & 11 & 26 & 11 & 33 & 51 & 31 & 9 & 27 \\
\hline $50-59$ & 1 & 6 & 7 & 16 & 8 & 24 & 41 & 25 & 5 & 15 \\
\hline 60$) \&$ Greater & () & 0 & () & () & 1 & 3 & 7 & 4 & 2 & 6 \\
\hline Total & 18 & 100 & 42 & 98 & 33 & 100 & 165 & 100 & 34 & 100 \\
\hline \multicolumn{11}{|l|}{ Gender } \\
\hline Female & 15 & 83 & 34 & 79 & 24 & 73 & 141 & 86 & 28 & 82 \\
\hline Male & 3 & 17 & 8 & 19 & 9 & 27 & 23 & 14 & 6 & 18 \\
\hline Total & 18 & 100 & 42 & 100 & 33 & 100 & 164 & 100 & 34 & 100 \\
\hline
\end{tabular}




\begin{tabular}{|c|c|c|c|c|c|c|c|c|c|c|}
\hline & \multicolumn{2}{|c|}{ District E } & \multicolumn{2}{|c|}{ District $F$} & \multicolumn{2}{|c|}{ District $\mathrm{G}$} & \multicolumn{2}{|c|}{ District H } & \multicolumn{2}{|c|}{ District I } \\
\hline & Freq & $\%$ & Freq & $\%$ & Freq & $\%$ & Freq & $\%$ & Freq & $\%$ \\
\hline \multicolumn{11}{|c|}{ Highest Degree } \\
\hline $\begin{array}{l}\text { Bachelor's } \\
\text { Degree }\end{array}$ & 10 & 56 & 12 & 28 & 4 & 12 & 32 & 19 & 2 & 6 \\
\hline $\begin{array}{l}\text { Master's } \\
\text { Degree }\end{array}$ & 7 & 39 & 15 & 35 & 18 & 55 & 78 & 47 & 17 & 50 \\
\hline $\begin{array}{l}\text { Rank I } \\
\text { Degree }\end{array}$ & 1 & 6 & 13 & 30 & 11 & 33 & 52 & 32 & 14 & 41 \\
\hline $\begin{array}{l}\text { Doctoral } \\
\text { Degree }\end{array}$ & 1 & 0 & 1 & 2 & 0 & 0 & 3 & 2 & 1 & 3 \\
\hline Total & 18 & 100 & 41 & 95 & 33 & 100 & 165 & 100 & 34 & 100 \\
\hline \multicolumn{11}{|c|}{ School Level Placement } \\
\hline $\begin{array}{l}\text { Preschool } \\
\text { Education }\end{array}$ & 1 & 6 & 0 & 0 & 1 & 3 & 1 & 1 & 0 & 0 \\
\hline $\begin{array}{l}\text { Elementary } \\
\text { School }\end{array}$ & 9 & 50 & 13 & 30 & 11 & 33 & 113 & 69 & 15 & 44 \\
\hline $\begin{array}{l}\text { Middle } \\
\text { School }\end{array}$ & 3 & 17 & 9 & 21 & 9 & 27 & 15 & 9 & 9 & 27 \\
\hline High School & 5 & 28 & 19 & 44 & 12 & 36 & 35 & 21 & 10 & 29 \\
\hline Total & 18 & 100 & 41 & 95 & 33 & 100 & 164 & 99 & 34 & 100 \\
\hline \multicolumn{11}{|c|}{ Instructional Content Area } \\
\hline $\begin{array}{l}\text { Self- } \\
\text { Contained } \\
\text { Classroom }\end{array}$ & 7 & 40 & 3 & 7 & 10 & 30 & 48 & 29 & 6 & 18 \\
\hline $\begin{array}{l}\text { Reading } \\
\text { Instruction }\end{array}$ & 3 & 17 & 4 & 9 & 2 & 6 & 12 & 7 & 5 & 15 \\
\hline $\begin{array}{l}\text { Language } \\
\text { Arts } \\
\text { Instruction }\end{array}$ & 0 & 0 & 2 & 5 & 4 & 12 & 13 & 8 & 2 & 6 \\
\hline $\begin{array}{l}\text { Math } \\
\text { Instruction }\end{array}$ & 1 & 6 & 7 & 16 & 3 & 9 & 15 & 9 & 4 & 12 \\
\hline $\begin{array}{l}\text { Science } \\
\text { Instruction }\end{array}$ & 2 & 11 & 7 & 16 & 3 & 9 & 12 & 7 & 0 & 0 \\
\hline $\begin{array}{l}\text { Social Studies } \\
\text { Instruction }\end{array}$ & 1 & 6 & 5 & 12 & 2 & 6 & 12 & 7 & 2 & 6 \\
\hline $\begin{array}{l}\text { Arts \& } \\
\text { Humanities } \\
\text { Instruction }\end{array}$ & 1 & 6 & 2 & 5 & 1 & 3 & 5 & 3 & 2 & 6 \\
\hline $\begin{array}{l}\text { Practical } \\
\text { Living } \\
\text { Instruction }\end{array}$ & 1 & 56 & 5 & 12 & 1 & 3 & 7 & 4 & 2 & 6 \\
\hline
\end{tabular}


District $\mathrm{E} \quad$ District $\mathrm{F} \quad$ District $\mathrm{G}$ District $\mathrm{H}$ District $\mathrm{I}$

\begin{tabular}{|c|c|c|c|c|c|c|c|c|c|c|}
\hline & Freq & $\%$ & Freq & $\%$ & Freq & $\%$ & Freq & $\%$ & Freq & $\%$ \\
\hline $\begin{array}{l}\text { Other } \\
\text { Instruction }\end{array}$ & 1 & 11 & 6 & 14 & 7 & 21 & 38 & 23 & 11 & 32 \\
\hline Total & 18 & 100 & 41 & 95 & 33 & 100 & 162 & 98 & 34 & 100 \\
\hline \multicolumn{11}{|c|}{ Years of Teaching Experience } \\
\hline 1-5 Years & 12 & 6 & 15 & 35 & 8 & 24 & 31 & 19 & 7 & 21 \\
\hline 6-10 Years & 1 & 61 & 7 & 16 & 4 & 12 & 41 & 25 & 8 & 23 \\
\hline $11-15$ Years & 3 & 6 & 6 & 14 & 20 & 61 & 22 & 13 & 4 & 12 \\
\hline 16-20 Years & () & () & 1 & 2 & () & () & 22 & 13 & 3 & 9 \\
\hline $21-25$ Years & 1 & 6 & 5 & 12 & () & 0 & 19 & 12 & 4 & 12 \\
\hline 26-30 Years & 0 & () & 4 & 9 & () & () & 17 & 10 & 3 & 9 \\
\hline $\begin{array}{l}31 \text { or More } \\
\text { Years }\end{array}$ & 1 & 6 & 3 & 7 & () & () & 7 & 4 & 2 & 6 \\
\hline Total & 18 & 100 & 41 & 100 & 33 & 97 & 159 & 96 & 31 & 100 \\
\hline \multicolumn{11}{|c|}{ Years of Teaching Experience in a YRE Calendar } \\
\hline $1-3$ Years & 10 & 57 & 16 & 37 & 8 & 24 & 39 & 24 & 10 & 29 \\
\hline 4-6 Years & 5 & 28 & 7 & 16 & 10 & 30 & 36 & 22 & 5 & 15 \\
\hline 7-9 Years & 3 & 17 & 10) & 23 & 9 & 27 & 44 & 27 & 8 & 24 \\
\hline 10-11 Years & () & () & 7 & 16 & 5 & 15 & 42 & 26 & 8 & 24 \\
\hline Totals & 18 & 100 & 40) & 93 & 32 & 97 & 161 & 98 & 31 & 91 \\
\hline \multicolumn{11}{|l|}{ Salary Range } \\
\hline $\begin{array}{l}\$ 20,001- \\
\$ 30,000\end{array}$ & 1 & 6 & 2 & 5 & 2 & 6 & 8 & 5 & 3 & 9 \\
\hline $\begin{array}{l}\$ 30,001- \\
\$ 40,000\end{array}$ & 12 & 67 & 18 & 42 & 13 & 39 & 49 & 30 & 15 & 44 \\
\hline $\begin{array}{l}\$ 40,001- \\
\$ 50,000(\end{array}$ & 3 & 17 & 12 & 28 & 11 & 33 & 70 & 42 & 13 & 38 \\
\hline $\begin{array}{l}\$ 50,001- \\
\$ 60,000\end{array}$ & 1 & 6 & 7 & 16 & 4 & 12 & 34 & 21 & 3 & 9 \\
\hline $\begin{array}{l}\$ 60,001- \\
\$ 70,000\end{array}$ & 1 & 6 & 2 & 5 & 2 & 6 & 1 & 1 & 0 & () \\
\hline $\begin{array}{l}\$ 70,001 \& \\
\text { Higher }\end{array}$ & 0 & 0 & () & 0 & 0 & 0 & 1 & 1 & ) & 0 \\
\hline Total & 18 & 100 & 41 & 95 & 33 & 97 & 163 & 99 & 34 & 100 \\
\hline
\end{tabular}

Note. $N=737$

Freq $=$ Frequency

$\%=$ Percent of the Particular District Total

$\%$ may not total $100 \%$ due to lack of some participant responses 


\section{APPENDIX Y}

Means, Standard Deviations and Correlations of Research Variables ( $N=748$ )

\begin{tabular}{|c|c|c|c|c|c|c|c|}
\hline Variable & M & SD & 1 & 2 & 3 & 4 & 5 \\
\hline \multicolumn{8}{|l|}{ School Climate } \\
\hline Collegial Leadership & 11.154 & 5.302 & & & & & \\
\hline $\begin{array}{l}\text { Professional Teacher } \\
\text { Behavior }\end{array}$ & 8.260 & 3.822 & $.54 * *$ & & & & \\
\hline Achievement Press & 8.868 & 4.7344 & $.48^{* * *}$ & $.64^{* * *}$ & & & \\
\hline $\begin{array}{l}\text { Institutional } \\
\text { Vulnerability }\end{array}$ & 14.098 & 3.991 & $-.25^{* * k}$ & $-.23 * *$ & $-.12 * *$ & & \\
\hline Climate Total & 56.556 & 10.452 & $.76^{* *}$ & $.79 * *$ & $.80^{* * *}$ & $.10^{* * *}$ & \\
\hline \multicolumn{8}{|l|}{ School Culture } \\
\hline Inquiry Practice & 8.855 & 3.531 & $.61 * *$ & $.67 * *$ & $.56^{\text {水* }}$ & $-.22 * *$ & $.70^{k * k}$ \\
\hline $\begin{array}{l}\text { Teaching Learning } \\
\text { Community }\end{array}$ & 8.126 & 3.048 & $.52 * *$ & $.79 * *$ & $.50 * *$ & $-.22 * k$ & $.67 * *$ \\
\hline $\begin{array}{l}\text { Collective Problem } \\
\text { Solving }\end{array}$ & 9.095 & 3.648 & $.67 * *$ & $.65^{* *}$ & $.55^{* *}$ & $-.32 * *$ & $.68^{* * k}$ \\
\hline Culture Total & 26.055 & 9.123 & $.68^{* * k}$ & $.78^{* * *}$ & $.60^{* * *}$ & $-.30 * *$ & $.76^{* * *}$ \\
\hline \multicolumn{8}{|l|}{ Teacher Efficacy } \\
\hline Student Engagement & 11.154 & $5.3(02$ & $.27 * * *$ & $.32^{* * *}$ & $.40^{* * *}$ & $-.21^{* * *}$ & $.35^{* *}$ \\
\hline $\begin{array}{l}\text { Instructional } \\
\text { Practices }\end{array}$ & 8.260 & 3.822 & $.26^{* * *}$ & $.30 * *$ & $.29 * *$ & $-.16^{* *}$ & $.29 * * \cdots$ \\
\hline $\begin{array}{l}\text { Classroom } \\
\text { Management }\end{array}$ & 8.868 & 4.734 & $.24 * *$ & $.24 * *$ & $.31 * *$ & $-.13 * *$ & $.29 * * k$ \\
\hline $\begin{array}{l}\text { Teacher Efficacy } \\
\text { Total }\end{array}$ & 28.283 & 12.115 & $.30^{* * k}$ & $.33^{* *}$ & $.39 * *$ & $-.19 * *$ & $.36^{* *}$ \\
\hline \multicolumn{8}{|l|}{ Collective Efficacy } \\
\hline $\begin{array}{l}\text { REGR factor score } \\
\text { for Group } \\
\text { competence }\end{array}$ & .000 & 1.000 & $.31 * *$ & $.39 * *$ & $.31 * *$ & $-.15^{* *}$ & $.36 * *$ \\
\hline $\begin{array}{l}\text { REGR factor for } \\
\text { Task Analysis }\end{array}$ & .000 & 1.000 & $.20 * *$ & $.34 * *$ & $.53^{* *}$ & $-.17 * *$ & $.37 * *$ \\
\hline
\end{tabular}


APPENDIX Y continued

Means, Standard Deviations and Correlations of Research Variables ( $N=748$

$\begin{array}{lllllllll}\text { Variable } & M & \text { SD } & \mathbf{1} & \mathbf{2} & \mathbf{3} & \mathbf{4} & \mathbf{5}\end{array}$

\section{Teacher Job}

Satisfaction

Teacher Job

Satisfaction Global

$25.644 \quad 8.137 \quad .59 * * \quad .53 * * \quad .43 * * \quad-.31 * * \quad .55 * *$

Teacher YRE

Satisfaction

$\begin{array}{lllllll}7.146 & 3.423 & .27 * & .27 * * & .31 * * & -.17 * * & .25 * *\end{array}$

Teacher

Satisfaction Total

$32.771 \quad 10.056 \quad .56$ ** $\quad .52 * * \quad .45^{* *} \quad-.31$ ** $\quad .53$ **

Teacher Intent to

Turnover

Intent to Turnover

Two Items

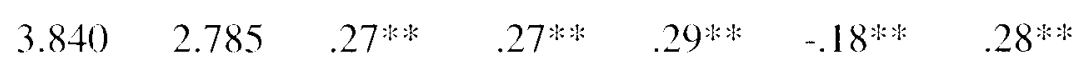

Intent to Turnover

Three Items

$5.864 \quad 3.768 \quad .26 * * \quad .24 * * \quad .27 * * \quad-.19 * * \quad .25 * *$ 
APPENDIX Y continued

Means, Standard Deviations and Correlations of Research Variables ( $N=748$ )

\begin{tabular}{llllllll}
\hline Variable & M & SD & 6 & 7 & 8 & 9 & 10 \\
\hline
\end{tabular}

\section{School Culture}

Inquiry Practice $\quad 8.855 \quad 3.531$

Teaching Learning

Community

$8.126 \quad 3.048 \quad .67 * *$

Collective Problem

Solving

$9.095 \quad 3.648 \quad .72 * * \quad .71^{* * *}$

Culture Total

$26.055 \quad 9.123 \quad .89^{* * *} \quad .88^{*} \quad .91^{* * *}$

\section{Teacher Efficacy}

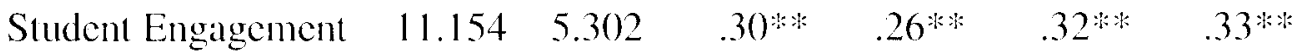

Instructional

Practices

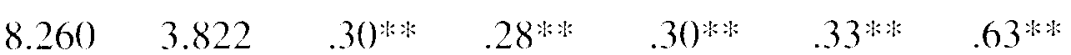

Classroom

Management

$8.868 \quad 4.734 \quad .26^{* *} \quad .22^{* *} \quad .26^{* *} \quad .28^{* *} \quad .65^{* * *}$

Teacher Efficacy

Total

$28.283 \quad 12.115 \quad .33^{* *} \quad .29^{* *} \quad .36^{* *} \quad .36^{* *} \quad .90^{* * *}$

REGR factor score

for Group

.000

$1.000 \quad .39 * *$

$.35^{* * *}$

$.34^{* *} \quad .41^{* * *}$

$.23^{* * *}$

competence

REGR factor for

Task Analysis

.000

$1.000 \quad .31^{* * *}$

$.28^{* *}$

$.36^{* *} \quad .35^{* * *}$

$.42 * *$

\section{Teacher Job Satisfaction}

Teacher Job

Satisfaction Global

$25.644 \quad 8.137 \quad .49 * * \quad .59 * * \quad .60 * * \quad .62 * * \quad .33 * *$

Teacher YRE

Satisfaction

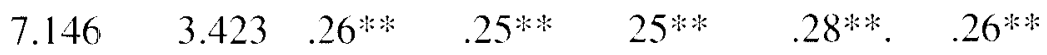

Teacher Satisfaction

Total

$32.771 \quad 10.056 \quad .48^{* *} \quad .56^{* * *} \quad .57^{* *} \quad .60^{* *} \quad .36^{* * *}$

\section{Teacher Intent to Turnover}

Intent to Turnover

Two Items

Intent to Turnover

Three Items

$\begin{array}{lllllll}3.840 & 2.785 & .25^{* *} & .25^{* *} & .31^{* *} & .31^{* *} & .23^{* *} \\ 5.864 & 3.768 & .23^{* *} & .24^{* *} & .27^{* *} & .27^{* *} & .21^{* *}\end{array}$




\section{APPENDIX Y continued}

Means, Standard Deviations and Correlations of Research Variables $(N=748)$

Variable

11

12

13

14

15

Instructional

Practices

$8.260 \quad 3.822$

Classroom

Management

$8.868 \quad 4.734 \quad .62 * *$

Teacher Efficacy

Total

$28.283 \quad 12.115 \quad .84^{* *} \quad .88^{* * *}$

REGR factor score

for Group

$.000 \quad 1.000\left(28^{* * *} \quad .23^{* *} \quad .28^{* * *}\right.$

competence

REGR factor for

Task Analysis

$.000 \quad 1.000 \quad .23 * * \quad .29 * * \quad .37 * * \quad .00$

\section{Teacher Job Satisfaction}

Teacher Job

Satisfaction Global

25.644

$8.137 \quad .33 * *$

$.33^{* *}$

$.38^{* * *}$

$.37^{* * *} \quad .30^{* * *}$

Teacher YRE

Satisfaction

7.146

$3.423 .27^{* * *}$

$.25 * *$

$3(0) *$

$.24 * *$

$.21 * *$

Teacher Satisfaction

Total

32.771

$10.056 \quad .36^{* * *}$

$.35 *:=$

$42 * *$

$.38 * *$

$33 * *$

\section{Teacher Intent to Turnover}

Intent to Turnover

Two Items

3.840

2.785 .17 ***

$.19^{* * k}$

$.23 * *$

$.17^{* * *}$

$.26^{* * *}$

Intent to Turnover

Three Items

5.864

$3.768 .16^{* * *}$

$.17 * *$

$22 * *$

$.17 * *$

$.23 * *$ 
APPENDIX Y continued

Means, Standard Deviations and Correlations of Research Variables $(\mathrm{N}=748)$

$\begin{array}{llllllll}\text { Variable } & M & \text { SD } & 16 & 17 & 18 & 19 & 20\end{array}$

Teacher Job Satisfaction

Teacher Job

Satisfaction Global $\quad 25.644 \quad 8.137$

Teacher YRE

Satisfaction

$\begin{array}{lll}7.146 & 3.423 & .41 * *\end{array}$

Teacher Satisfaction

Total

$32.771 \quad 10.056 \quad .95 * * \quad .67 * *$

Teacher Intent to Turnover

Intent to Turnover

Two ltems

3.840

$2.785 \quad .54 * * \quad .30 * *$

$.55^{* * k}$

Intent to Turnover

Three Items

$5.864 \quad 3.768 \quad .50 \% * 39 * * \quad .53 * * \quad .91 * *$

Note. Variables Number Identification

$1=$ Collegial Leadership

$3=$ Achievement Press

$5=$ Climate Tolal

$7=$ Teaching Learning Community

$9=$ Culture Total

$11=$ Instructional Practices

$13=$ Teaching efficacy Total

$15=$ Task Analysis

$17=$ Teacher YRE Job Satisfaction

$19=$ Intent to Turnover - Two Items
$2=$ Professional Teacher Behavior

$4=$ Institutional Vulnerability

$6=$ Inquiry Practice

$8=$ Collective Problem Solving

$10=$ Student Engagement

12 = Classroom Management

$14=$ Group Competence

$16=$ Teacher Job Satisfaction Global

$18=$ Teacher Job Satisfaction Total

$20=$ Intent to Turnover - Three Items 


\section{APPENDIX Z \\ Teacher Survey Comments for the Open-ended Survey Item}

For me, personally and professionally, the year-round education calendar has ....

- The Year-round calendar makes teachers more motivated to teach because they are getting lots of little breaks to rejuvenate themselves. The biggest plus is with the students they have time to rejuvenate too but not so much time that they forget the skills taught.

- I have learned to enjoy the ycar around calendar. I think it is good for teachers but I am not sure it is good for students as I think they need more time in the summer.

- Works well, however, for A.P. students who test in the first week in May it is too short.

- Given me restful time off to enable me to be fresh and ready to begin cach new quarter.

- I am supportive of the year-round education calendar as a teacher and a parent. I feel as though the calendar allows opportunities for students to do enrichment and reinforcement activities that improve student learning.

- I like the year-round cducation

- It has some benefits. It seems to break up the year well. I'm not sure how well the intersession helps; this is my first year teaching in this system. Personally, I would prefer a longer summer. Students who are behind could go to summer school.

- The year-round education calendar has allowed me as a teacher, to "recharge" my batteries and take time to reflect on my students' performance and to make any necessary changes I feel I might need to better my students and enhance me as a teacher.

-

Been beneficial for both students and staff. It has allowed for professional development activities that perhaps a traditional calendar would not allow for.

- Been a blessing. I find that the teachers and students are refreshed after the breaks. Also, it reduces burnout as I know I am always approaching a break. I like to say that I can handle any class 9 weeks at a time!

- Been effective in diminishing teacher and student burn-out. That in and of itself is a worthwhile bi-product.

- A good experience. I like having definite time frames to allot the work. having a break between the nine weeks gives me a chance to review what was covered and make any changes I think will help

- A great success. 
- A new experience and I like it

- A wonderful addition to our district. I enjoy the breaks after each grading period, and I use them to spend time with my family, to reflect on what's going on in my job and 10 plan ahead. I think the students of all ages need a break to just be kids.

- Absolutely Been wonderful. The breaks come at just the right time for the teachers and the students. They allow the teachers to finish out the nine weeks, analyze success and failures and prepare for the second nine weeks.

- advantages: 1.) You can stand just about anything for nine wecks, knowing you have a two week break at the end. 2) The students AND teachers enjoy the breaks, especially the Fall break. 3) We offer remediation to students with low averages.

- Advantages and disadvantages. After being on this calendar for several years, I feel there is never any down time as a teacher. I return to the classroom to prepare it for the next year the 3rd week in July or even before.

- Afforded opportunities for children to go 10 enrichment and intercession classes during the 2 week break. It also shortens the summer, so that students will not lose what they have learned. I LOVVE the year-round calendar!

- After cach term, there is a small break to allow time for staff and students to regroup. The students don't seem to lose as much content knowledge with shorter breaks.

- Aided me to be the best teacher I can be. I use the breaks to refresh and plan for the next semester. It has been greal!

- All of the components of successlul teaching and learning ONLY IF intersessions are implemented and supported financially by the board.

- Allowed a more consistent calendar and time to work with my students. I feel students have less time off to forget things.

- Allowed an opportunity for the students to have a break and return refreshed, with a renewed sense of enthusiasm.

- Allowed for a better continuum for learning. I like the frequency of the breaks and their lengths.

- Allowed for greater flexibility in my instruction and provide the opportunity for little breaks that are my re-charging time, which has boosted my efficiency and attitude about my job.

- Allowed for more family time at home throughout the year. It has allowed for needed breaks for the students after nine weeks or so of intense study. It spaces out the year nicely for students and allows for extra help during intersession if they need it.

- Allowed for more time with my family at different times of the year and has given me more energy to begin a new nine weeks term. I feel more rested and ready to tackle a new group of students.

- Allowed for student and staff "down time" throughout the school year. This allows for both students and staff to become refreshed and ready to go again. However, I am dissatisfied with the amount of in-service flexibility. 
- Allowed me personal and professional flexibility. I love it!

- Allowed me the necessary time to rejuvenate myself after going $100 \%$ for nine weeks. I don't find myself totally exhausted by Winter Break or Summer Break. It has resulted in a lew missed summer PD opportunities/summer classes that conflicted with the late end of school.

- Allowed me the time between semester to be refreshed and ready to return in addition to allowing me the extra time to plan better activities.

- Allowed me time to rest and recoup, while still having time to get into my room to make some changes that benelit my students leaming.

- Allowed me to get into a teaching flow that feels less interrupted.

- Allowed me to have more time with my students to affect them on a personal level. The only draw back I have found is that it prohibits me from getting involved in some summer institutes because the schedules clash.

- Allowed me to plan activities for the children that can be presented before a short break and expanded upon when they return with little review lime. The year-round education calendar has allowed me as a teacher to stay fresh and prepared cach day.

- Allowed me to recharge my batteries. Students always seem ready for the break when it occurs. Afterward they seem to be recharged, rested, and ready for new learning. I have had a break and time to make fresh decisions about learning strategies that will help students.

- Allowed me to renew my energy and enthusiasm during our two week breaks that oceur every nine weeks. Year-round calendar has made me condense my units so that nothing is carried over from one quarter to the next. It has reduced the amount of time I have I have to review.

- Allowed me to rest, regroup, and return from fall, winter, and spring breaks revitalized and ready to give it my best again. It allows the year to be broken up into 4 quarters of teaching content instead of betore Christmas and after Christmas.

- Allowed me to teach more years. No long periods of time in school without a break. Kids have a better attitude because we have breaks.

- Allowed me, as well as the students, to reenergize our body and minds during our two week breaks. Our calendar also allows me to make extra money working intersession and gives students the opportunity to get caught up in skills where they are falling before.

- Allowed my students to focus more intently during each quarter, knowing that at the end of that quarter they will have a chance to take a break and relax, before coming back to school to start a new quarter.

- Allowed students the opportunity to get extra support during Intersession. Also, students and teachers are allowed breaks to "recharge" themselves during needed times throughout the year. I love year-round school! It meets students' learning needs. 
- Allowed students to achieve. They often forget knowledge during long summer months and get into trouble because they have too much time on their hands.

- Allowed students to maintain skill development, motivated students because of the breaks, allows teachers and students to have more family time together,

- Allowed the students to retain more information. I believe the long breaks provide lower achieving students to forget much of what they have learned. Personally when the breaks come around I need one as well.

- Allowed us to chuck learning times and break times. We work very hard during each nine week period knowing we are going to get a two week break. By the end of the 2 weck break we have caught up and rested and are ready to get back to work.

- Allowed us to have a much needed fall break and breaks the year up more. Both the teachers and the students have adjusted well to this calendar.

- Although we have scheduled two week breaks, the amount of work that we are required to do involve spending a lot of our break working, whether it be in the classroom or at home.

- As with most things, it has its good and bad points. My experience is that traditional calendars and traditional school days (that is, non-blocked) are more effective. However, I think that either system can work, if everyone is motivated to make it work

- Aside from the long stretch in February/March, I think the breaks come at just the right time for both students and teachers. It keeps both from being "burned out".

- Been a benefit to both students and teachers, cnsuring that the breaks are long enough where both parties feel well rested and come back ready to learn, yet not so long (particularly over summer) where students are out of an educational environment.

- Been a blessing! After the breaks we enjoy, both the students and the teachers come back rested and fresh, ready to learn new things. I would NEVER choose to go back to the "traditional" school calendar. Students have remediation and cnrichment opportunities.

- Been a breath of fresh air. The breaks in the fall, winter, and spring and summer give u enough time to regroup and go again. The students do not seem to be as tired and weary during the year.

- Been a challenge when my children were younger and in other school systems because we never had the same schedules. Also we are a farm family and coming back to school in at the end of July was a hardship on my role as a farm wife.

- Been a challenge. I find it hard to find stopping points in my lessons when breaks are about to happen. If I am in the middle of a chapter I have to go back and almost start over because the students don't remember from 2 weeks ago.

- Been a good change for our school system. The students are really refreshed after two weeks off and come back with a good attitude. Plus, it really feels like each quarter represents a fresh start when you separate them with two weeks. 
- Been a good change. I have enjoyed teaching my students during the yearround calendar. I personally like having some time off during each season. I have seen very little educational/behavioral disadvantage to the year-round calendar with my students.

- Been a good experience, however, I do not like getting out of school in early June, I do not feel you should ever be in school past Memorial Day.

- Been a good experience. Discipline problems seemed to decrease when we started this calendar. By the time the students and teachers were tired of each other, we got a break. Then we both came back with a better attitude.

- Been a good experience. I do like having the frequent breaks to look forward to during the year. It is a motivating factor to have breaks and vacations at regular intervals for both students and teachers.

- Been a good experience. I like the chunks of working time both professionally and personally. I think the instructional segments are beneficial and the breaks come at a welcomed/needed time for all involved.

- Been a good fit for me as far as getting rejuvenated during the long breaks. I personally don't think school should be in session after Memorial Day weekend. Being in school during June is a waste of time along with the three intersessions that we hold.

- Been a good thing. I teach special needs children and the year-round calendar offers them less time over the summer to forget skills they have learned. The breaks offer the students enough time to start $10 \mathrm{get}$ bored and when they return they are ready to learn.

- Been a great and rewarding schedule for the students and teachers at our school.

- Been a great opportunity. I would definitely be upset if we thought about changing it. I think the students and teachers really need the break in the Fall. It is overall a win-win situation for everyone involved. I have nothing negative to say about the calendar.

- Been a great schedule for my students' retention rates and fine for my "recovery" periods. I like our current schedule; it has just been a difficult experience with our lack of effective leadership.

- Been a great way to "break up" the school year and help the students retain more information throughout the breaks.

- Been a huge benefit! I love the vacations and I am thoroughly enjoying the time I get to spend with my family during the vacations. The students also do very well with this calendar, and academic achievement is high.

- Been a positive expericnce

- Been a positive experience because just when the students are beginning to be uncooperative in groups and not get along, it is time for a break. When we return from break most of the students are refocused and ready to participate better.

- Been a positive experience. 
- Been a positive experience. I like having a break between each 9 weeks. It helps the students to better understand the time they have for each grading period when they have a break between the grading periods.

- Been a positive experience. I really enjoy the breaks and I think they are good for staff and students alike.

- Been a positive experience.

- Been a professionally and personally rewarding experience!!!

- Been a rewarding experience with advantages over the traditional calendar.

- Been a success. It gives us more time to participate in functional activities that require extra time. The breaks are beneficial because it gives the students enough time off that when they return to school they are ready to work again.

- Been a wonderful asset to my family because I feel I spend more time with them throughout the year rather than just during a long summer. It is also great professionally because the students come back from our breaks realy to get back in the swing of thinking and learning.

- Been a wonderful change! I feel that this calendar allows just enough "breaks" to recharge everyone's batteries, students and teachers. I think that students need time off and so do teachers, but are ready to return and begin anew in spirit.

- Been a wonderful change! I feel that this calendar allows just enough "breaks" to recharge everyone's batteries, students and teachers. I think that students need time off and so do teachers, but are ready to return and begin anew in spirit.

- Been a wonderful experience for both myself and my students.

- Been a wonderful experience. The three week breaks are just enough time to refresh myself both personally and professionally. This calendar keeps me from reaching burn-out, just when I am getting to the point where energy is beginning to flag.

- Been a wonderful! I love that I get to take breaks to rest emotionally and physically. It helps me to rejuvenate so my creative juices kecp flowing. Not to mention that it gives me more time to plan curriculum for current students and future students.

- Been an asset in the sense that it keeps the kids actively involved within the school without large lapses in education, school policies, and the school environment.

- Been an effective tool to enhance students learning through ESS intersession.

- Been beneficial and enjoyable

- Been beneficial for both students and staff. It has allowed for professional development activities that perhaps a traditional calendar would not allow for. 
- Been beneficial for both students and teachers. The breaks throughout the school year help the students to have fun at home and then they are usually ready to come back to school to learn. This also seems true for the teachers/staff as well.

- Been beneficial for me. I must admit that I would like to give up a week of break to be able to start later in August.

- Been beneficial for planning and re energizing. By having a year-round calendar the students and teachers don't get burned out as easily. We work hard for that 9 week period and then take a short break so we can get ready for the next 9 week period.

- Been beneficial in allowing students and teachers the opportunity to have periodic breaks to refresh themselves and return to their academic work.

- Been benclicial to me both as a parent and as an educator. I believe the breaks are good, both lor the students and the teachers, because they allow adults and students to take some needed time off to reflect and be refreshed about learning and leaching.

- Been beneficial to me personally and professionally. The overall gist of a yearround calendar boils down to you start school two weeks carly, and you take ofl two weeks in October. The weather in August is hot and humid, and the weather in October is great.

- Been beneficial to student learning and instruction

- Been beneficial, it's good to have the breaks to rest and re-energize.

- Been beneficial. The four quarters are great for organizing the core content for teaching and reviewing. Burnout is not as much of a factor for teacher and student. This is such a positive for being able to teach in a upbeat manner-most of the time.

- Been disappointing. The true year-round was disruptive. The more moderate modified calendars of the last two years have Been much better.

- Been effective for students that seem to regress during the summer break.

- Been extremely important, not only for me but for the students also. Our calendar provides excellent opportunities for students to attend enrichment or "fun remediation" activities during the second week. Having 2-week breaks also allows flexibility for reflections and renewal.

- Been fantastic for learning and teaching. The breaks give students and teachers a break while providing extra learning time for those who need it.

- Been fine with me.

- Been fine. You need to understand, however, that the calendar has presented really no problems. Any negativity in my responses stem from having five changes in administrators over the past 7 or so years. We have also changed instructional schedules. 
- Been fine. The breaks throughout the school year are nice and our summer is not that much shorter than a typical summer.

- Been good

- Been great for my family. We get to travel to see our relatives in other states during our breaks off. We also don't have to take sick days to go to the doctor because we can also do that during our breaks.

- Been Great!

- Been great, I love the 2 week break. It seems to hit just when I'm running out of energy.

- Been great. My husband and I live a good distance from our families and we use the breaks to travel and visit our families. I feel the breaks help rejuvenate the students as well. They seem much more ready to learn when we return from a break.

- Been great. I enjoy the breaks-and so do the students.

- Been great. My family and I love being able to take a vacation during the fall and sometimes in the spring. It's the perfect time to travel to see our son in D. C.

- Been HEAVENLY. The 2 weeks off after each quarter refresh not only the students but the teachers. It is a WELL needed and WELL deserved break.

- Been interesting. I like it a whole lot better than where I worked at before. I try to encourage my student to always study and hang in there for that all 100 important semester. I wanted to cover all the material that I was covering with the year long approach.

- Been mostly positive, but I don't honestly see it making much difference in student achievement or teacher job satisfaction. I like having substantial breaks after each 9 weeks of instruction, and I don't mind coming back to school a couple of weeks early.

- Been much better than the other calendar. The two week breaks give teachers and students a chance to relax and enjoy their families without forgetting about school altogether.

- Been nice. I really like the breaks and find them generally timed well. I feel that they refresh everyone.

- Been of a great advantage. I enjoy having the much-needed breaks spread throughout the year. I don't mind going to school earlier in the summer when I can have breaks throughout the year.

- Been okay. I don't really have any other education calendar to compare experiences. I think the year-round calendar has its advantages and disadvantages just like anything you look at. I believe students like the break and working in a school such as ours.

- Been one of the advantages of my job. The regular two week breaks help refresh teachers and students. 
- Been positive.

- Been positive. We have gone back to a more traditional schedule because of a lack of participation in the classes held during the break, having to go into June, and we would rather more instructional days before testing.

- Been pretty nice. I really enjoy the two week breaks every now and then. I dislike only having two months for the summer break. I do however believe for educating the students, and for their effort to learn, the year-round calendar is great.

- Been refreshing in providing breaks throughout the school year for teachers and students. It has also made it easier for student learning, as retention is better with the breaks being spread out and not having such a long time off between grade levels.

- Been the only calendar in which I have taught so it is all I know. I work for a terrific school; I just worry about student discipline within the school.

- Been the only calendar that I've worked in. For that reason, I can't compare it to the traditional calendar from a teaching point of view. Seems OK.

- Been very advantages for all. First, it allows my students to continue in their academic progress. With a shorter summer break, my students of moderate to sever disabilities do have as much regression in their academic goals.

- Been very beneficial for both students and teachers.

- Been very beneficial for my students as well as personally allowing me to spend more time with my own children more during the school year.

- Been very beneficial to me and students. I like having breaks every nine weeks because it gives me and the students at our school the opportunity to spend some refresher time away from each other.

- Been very effective. The break between quarters allows the students to take a break and rejuvenate for more learning the next quarter.

- Been very successful

- Been very successful. I enjoy the longer breaks we receive. The students seem to better retain previously learned information. Personally, I do like the yearround education.

- Been very successful. Parents and teachers alike that I have had contact with are pleased with the year-round school calendar that we use in our district.

- Been wonderful

- Been wonderful for the parents, students, and teachers.

- Been wonderful! We are in school for 9 weeks and then off for 3 weeks and I love it. We are able to "chunk" our time (teachers and students!). It is also great for setting quarter goals as opposed to semester goals. I would recommend our calendar to other schools. 
- Been wonderful. It seems that the students (and teachers) receive a short break before you begin to the feel the "burn out". The students come back refreshed and ready to pick back up where we left off. Also, there is less review time to do in August.

- Been wonderful. We have a break right when teachers and students begin to get burned out. After they come back from break, they are ready to learn. Planning is better for the year. You know what has to be done each quarter.

- Bcen wonderful. I don't know how we made it with the traditional calendar. The teachers and students need breaks from each other, so why not spread out the school year to include breaks? Planning is easier.

- Been wonderful.... there are so Many advantages to this calendar, both professionally and personally. I would not be happy to go back to a traditional calendar.

- Been wonderful. I love the breaks in the calendar and the students do as well. I don't think that it disrupts the students leaning by having breaks in the school year, but on the contrary helps the students to be motivated. It allows everyone to get a break.

- Benefited both me and my students.

- Benefited students and teachers. Students who have longer periods of time away from academic instruction have trouble retaining the previous year's curriculum. Teachers notice this significant problem within the YREC.

- Benefits and disadvantages. The Two week break in the Fall and Spring gives the students an opportunity to rest and plan vacations with their families. It also provides intercession which allows students to get extra instructional throughout the year vs. traditional calendars.

- Benefits for students shorter breaks from school more learning time for them. The summer may be shorter but I truly believe that it helps the students to retain some information learned from the previous year.

- Benefits for teachers and children. More flexibility and more opportunities.

- Benefits in that it helps students achieve who haven't achieved in regular school. JW, we are an alternative program which must go an extra 35 days (4 hour days) as we are DJJ and KECSAC.

- Benefits in the form of enabling periodic breaks for both the students and the teachers.

- Boosted morale. I love it and would not work at a school without it.

- Both positive and negative sides

- Broadened the range of experiences I can present to my students. It allows for real life connections to what is taught making the content meaningful, therefore, more likely retained. Coming from a traditional calendar to the year-round has rejuvenated me. 
- Broken down into quarters I do think it gives the students a break, so that they may come back refreshed and start the next quarter. I haven't seen much lost, as far as, the content goes with the teaching.

- Built morale. It has allowed students periodic remediation and /or enrichment.

- Can be difficult to take college classes in the summer, because of the schedule.

- Caused less of a burn out feeling. The breaks are refreshing for the students as well as the staff. I think the mini breaks instead of one large summer break also helps with retaining info. I enjoy the fall break very much.

- Caused me problems in finding summer work to supplement my teaching salary and also made it difficult to complete Rank 1 requirements through summer school (university) courses.

- Caused me to have to work during the time we have off...some of our outside events take place at a time that causes me to have to prepare students while they're on the break.

- Classified employees are not given a chance to go to PD or get promotes as the certified employees. A Gap that needs to be reviewed

- Created blocks of time for learning followed by needed rest, reflection and renewing of my vision. In addition, the students receive the same with the added benefit of remediation and enrichment if they choose.

- Created conflict with my family. I teach at a different district than my children. The school calendars sometimes do not match, and planning a vacation can be difficult.

- Created less burn out for me but I fill our children need more classroom time. It seems like when you get them settled in a routine.... they have a break and you start over again.

- Currently, I work on a 230 day contract with my preschoolers coming an extra 40 days per year because of a 3 year ERF federal grant. However, my school is on the alternative calendar which I was a part of for 5 years.

- Discouraged my interest in returning to college to work on other certifications. I feel like I have no summer break to recharge after the end of the school year. I do not want to go to conferences or workshops during the summer because I lose time at home.

- Does not work as well for Kindergarten students. The fall break is too early in the year. They need to keep the consistency of a routine and schedule. It takes too long to get them back into that routine when we return.

- Enabled Me to be an effective teacher with opportunities for breaks so that I may come back fresh, alert, and excited about working with young people.

- Enabled me to better balance life and work, to intervene when a student falls behind, and to improve my teaching by using part of my breaks to reflect, plan, and prepare.

- Enabled me to have the time with my daughter that I desire. I get out of school at the same time as she does; I have the same vacations and holidays etc. Being in the school system has afforded me exactly what I was seeking in terms of my 
commitment to my profession.

- Enabled me to more effectively plan and provide instruction that has a focus and motivates my students. The students are reenergized after fall and spring breaks. I would never want to go back to the traditional calendar.

- Enabled me to teach in a school with a staff that for the most part is all positive. Both the teachers and the students do not seem to get "burned out" as fast due to the frequent breaks. Also students seem to retain information taught from one grade to another.

- Energizing capabilities for students and teachers; renewal and revitalization opportunities for students and teachers time to wrap things up; time to plan; time to relax for everyone -one week for each!

- Enhanced job satisfaction. It really helps to be able to regroup, rest, and plan with breaks occur after each nine weeks.

- Enhanced my professional and personal life. I was part of a year long study group for an alternate calendar and voted against it because there was little data to support student achievement. I WAS WRONG!!!!!!!!!!

- Enough breaks for the students not to get burned out, and still able to focus on school.

- Enough breaks spaced throughout the school year to allow students to be fresh and rested. As soon as students begin to be burned out or feel overwhelmed then we have a short break. It is very nice and keeps the curriculum moving and keeps school from being boring.

- Excellent opportunities for students to work on areas they are behind, during the breaks. It gives students and teachers a chance to "recharge" every 9 weeks to help with "burnout". It also gives students, teachers and their families more opportunities for more vacations.

- Extreme benefits. students are Given breaks throughout the year and they do not get burned out

- Flexibility of scheduling and constant reinforcement of concepts during breaks.

- For me professionally the YRE has helped my students retain what they have learned. Also they are not out of the routine too long and seem to adjust quickly when they do return. For me personally, I feel that the YRC breaks up the year just enough to keep student focused.

- For me the year-round education calendar has been good. It allows me the opportunity to take a break and allows me time to prepare for future lessons.

- Given appropriate breaks when needed for students and faculty.

- Given flexibility to teaching. Provide breaks at needed times for students and teachers. Provide time for students who are lagging behind to catch up. Helped students and teachers to avoid burn out.

- Given lots of opportunities for extended school services that would not be available if we were under a traditional calendar system. 
- Given me a break to look forward to. We work very hard while at school and the 2 week breaks come at a good time. The students are mentally ready for a brain break. We then come back and work hard. The eight weeks during the summer is enough to feel rested.

- Given me a chance to recharge my batteries four times a year, Provide extended school service and tutoring possibilities for students, spread learning opportunities out over the year.

- Given me a chance to refresh my energy and enthusiasm during our break time every 9 weeks. It has made me condense my units to fit into a 9 week period of time because you cannot carry over a topic with a two week break in the middle.

- Given me a chance to take a break, refocus, and re-energize during the year; also to do more long-range planning for each upcoming quarter. Personally, with children in the home, it makes it hard to do all the things they and I want to do as a family during the summer.

- Given me a chance to take more vacations throughout the year!

- Given me a much needed two week break 1 for family and 1 for catching up on household routine needs

- Given me an opportunity to have a short break and come back rejuvenated and ready to motivate students to learn. The students are also ready to learn again from the short break but have not forgotten material like they would with longer breaks.

- Given me an opportunity to refresh and revamp

- Given me and my students a much-needed and sensible break at the end of each nine weeks to recharge and re-equip. The additional days in the calendar are worth the breaks in between.

- Given me better and more frequent opportunities for travel and family visit

- Given me flexibility and more job satisfaction. I really like it!

- Given me greater flexibility. It reduces teacher 'burn-out' and fosters positive attitudes among teachers. Spirits are more even throughout the year and teachers' tempers are better, attitudes more positive. I would not want to consider teaching in the traditional calendar.

- Given me more flexibility to manage my personal and professional life and has Helped my students retain more of what they have learned the previous year.

- Given me more freedom and allowed me and my children, whom are students in this district, a well enjoyed and needed break from the rigors of school at different times of the year.

- Given me more frequent breaks when the school year becomes stressful there is a break and we all come back refreshed and ready to learn again. The children seem to maintain a higher level of motivation because of the frequent breaks. 
- Given me more opportunities to extend the learning environment into the summer.

- Given me more time during spring and fall break. It is fine. Sometimes I wish I could get out of school earlier in May and start a week later in August, but then I would lose my 2 week breaks, and I do not want to do that.

- Given me much needed breaks from the stress of dealing with children on a daily basis. It also gives me time to help my extended family with health care problems.

- Given me opportunities to assist students during break times to bring up their grades. It also allows us time to have extra fun activities during the breaks. It helps reduce the burnout of students and teachers by giving us breaks in between quarters.

- Given me the break I need to refresh my body and mind. My school is competitive and the principal sets high expectations and therefore a high work load so the break is necessary. The students need a break as well because our focus is so extremely content oriented.

- Given me the breaks that I have needed to refresh myself to be ready to start again and do my best with each quarter.

- Given me the opportunity to execute my core content more effectively, and allows students ample time to absorb the knowledge. I feel that the students have adequately-spaced breaks, allowing them to regroup for the next section of the school year.

- Given me the restful breaks I need, when I need them, to be more effective when I am in the classroom. The breaks from school tend to be between terms, and make more sense in the educational scheme of things.

- Given more structure to the school year and, with more frequent breaks, allowed me to take the time to relax and renew my enthusiasm for teaching throughout the year.

- Given much needed breaks when teachers in other schools are getting burned out. I am able to do more professional development throughout the year where I don't have to take days off from my job. I come back more energized and have time to get caught up.

- Given my students and me opportunities to rest and rejuvenate myself after each grading period. It Is always difficult to get started back after a break.

- Given needed breaks when students and teachers are ready for them. It has allowed all of us to rest, relax, and refocus on what needs to be done. We get our breaks right about the time we really need them.

- Given students and teachers a break right about when it is needed. It allows intervention through intersession to make a difference with those struggling students. There is also a bit shorter summer break than the traditional calendar.

- Given students and teachers good breaks of time during the year to refresh and renew, while giving students a more constant calendar between breaks to practice and polish skills. The breaks provide consistent times for students to receive extra. 
- Given students extended school service opportunities that would not be available under a traditional calendar system.

- Given students, faculty and staff more breaks throughout the year, even though shorter; given students-in-need the opportunity to attend intersessions and possibly reach proficiency; allowed students to retain more academic information.

- Given teachers and students the "down time" in between quarters to relax and revitalize and travel or attend professional workshops.

- Given the students and teachers the breaks they need to come back to school ready to learn. Allows me as a teacher to take part in various professional development activities throughout the school year. It also allows for teachers to come into the building to set up classrooms.

- Given the students in this county the best chance to succeed. They are able to use "breaks" to their advantage, if needed. Approximately every nine weeks, students receive a two- week break, one week of which is used for an intersession period.

- Given us the needed mental breaks in the fall and has given teachers and students the time to rest. I would hate going back to traditional calendars.

- Given very needed breaks at opportune times during the year!!

- Giving me more regular break time (Oct. and March) to reflect and refine my teaching throughout the year.

- Good benefits. I like the flexibility of the breaks during the year. The extracurricular classes offered during the two week breaks are educational but fun.

- Great

- Great advantages - the breaks make it possible to hold "camps" for the kids so they can work on academic skills outside the classroom.

- Great advantages for student support and remediation. When focusing on formative assessment it makes sense to me that we not only practice this in our classrooms, but also with our school calendar.

- Great benefits due to flexibility in scheduling. The calendar provides a chance to renew and plan and gain opportunities to improve teaching strategies.

- GREAT benefits for children that need extra time to learn. More flexible time for vacations and allows teachers to feel refreshed after working hard for 9 weeks.

- Great benefits- one) I have time with my little boy to take vacations, etc. two)it seems to give breaks when students and teachers are feeling the stress of the school load and need time away from the school setting.

- Great benefits. Gives breaks for teachers and students to be away from school and start fresh when the break is over. Still gives plenty of time for summer vacations and fall vacations.

- Great. I enjoy the breaks off. I think students retain information over the summer better. 
- Has Been very nice. The fall break is the best thing about the calendar.

- Has Helped attendance, behavior, teacher moral, and motivation from the students.

- Has helped me learn about different teaching methods associated with this schedule. Benefited me in giving breaks more frequently. Helped me adapt to time frames.

- Has made my overall teaching experience a success. I love the breaks, and most kids are ready to come back in the summer anyway. Kids don't forget their routine of school after the breaks and the content is still fresh.

- Has not made that much of a difference in what I do

- Has worked very well overall. I only concern is that my wife is a teacher and she is not on the year-round schedule and this creates problems due to us not being off at the same time to take vacations, etc in the spring and fall.

- Helped break up the year and organize learning activities in a way that will ensure child improvement.

- Helped Many of my students to retain information that would be lost over longer periods during the summer.

- Helped me professionally get well-rested for new quarters. Personally the yearround education calendar gives me more of a chance to spend more time with my family throughout the year.

- Helped me to get my plans together as a new teacher teaching five different classes. The breaks in between quarters are great to get things done and take a break when you think you are about to lose your mind. It also gives students an opportunity to master learning.

- Helped me to plan my year more effectively. We create a syllabus for each quarter and I really like that. The week of Excel at the beginning of each break is a very valuable time for underachievers to get extra attention and skills.

- Helped my students not to lose so much of what they have learned over the long summer breaks. It has Helped my personally and professionally to have some time to regroup and come back in a new quarter refreshed and ready to go after the long breaks.

- Helped students retain skills while benefiting from the breaks.

- Helped the children retain what they learned the previous year.

- Helped to establish a school in which the negative effects of home and home life can help to be nullified by letting the children remain in a constant positive environment and the calendar also helps us to not have to set up a new school routine every year.

- Helped with moral - both teacher and student. Just when we all get tired and stressed, we have a break and come back ready to go.

- I actually like the YRE and feel that not only do the students need the breaks but, the teachers also do. 
- I am not on the year-round calendar, but I wanted to see how this survey worked.

- I believe that the year-round school calendar gives students the breaks that they need from school, but at the same time, the breaks aren't too long (especially summer break) where the cobwebs will form and material and skills will be lost.

- I believe the year-round calendar presents many challenges for students who are involved in athletics and extra-curricular activities. Those students are certainly losing more of their "break time" than other students. The same can be said for teachers.

- I do not feel qualified to answer this question as I have not worked long enough in this school district to experience the year-round calendar.

- I do not feel that there is any difference between the schedules. The long breaks make the beginning of school arrive multiple times during the year. Your routine and discipline has to be reestablished. The flow of learning is also broken.

- I do not like the year-round calendar at all. We are generally off for two-three weeks at a time. This is way too much time to be off. When the students get back from break, it is difficult to get them back on task and ready to work.

- I do not really care for our current calendar. I enjoy having time with my family away from work. With our present calendar, summer break is about 6 to 7 weeks in length. My school always mandates 2 to 3 days of PD during this time.

- I don't care for it from the standpoint of the teacher, there are too many breaks thrown in that stretch everything further apart. Yes, the breaks are nice, but I feel like the kids get out of the swing of things every time we have two week break.

- I don't feel like the calendar has been a plus for me as much as it has Been for the students. I am still teaching the same number of days; they are just spread out differently. I think it enhances students' ability to retain knowledge by having several breaks and intersessions.

- I don't feel that it is all that different. We still get the breaks that are needed and the students get the instruction that they need. I like the year-round calendar. I think it is better here then it was at the last school system I was in.

- I don't see a big difference in student achievement and the year-round calendar. Our calendar is not a true year-round calendar (on 9 weeks off 3 weeks). I think this calendar is very difficult for teachers, who live in another district and who are parents of children in other districts.

- I enjoy having the breaks with my family to accomplish jobs around the house that are bigger than a weekend job. It is nice to have a vacation on the off peak times for theme parks.

- I enjoy our 2 week breaks away in order to refocus and recharge myself to be a more effective, enthusiastic teacher. I often notice a decrease in skills and performance ability (in practice, application, and assessment) upon returning from our 2 week break. 
- I enjoy our two week breaks. I am able to refocus and recharge myself to be a more effective facilitator of instruction. I do often notice a decrease in skills and performance ability among students (in practice, application, and assessment) upon returning.

- I enjoy the break in the fall an spring. It provides an opportunity to visit with farmers at harvest time.

- I enjoy the breaks when they come, but I'm always glad to get back. It is wonderful that we have an intercession during the breaks to help the students with low grades sometimes it is difficult to start classes so soon, but once started it is okay.

- I enjoy the mental break we receive during the fall and spring. I think the children retain more information being home for a couple of months in the summer. I would recommend this calendar to others.

- I enjoy the year-round calendar. I come from an area in the country that does not adopt this calendar, probably never will. I enjoy the October and Spring Breaks... as I am able to unwind with my family and children and still travel, as I have family spread across the state.

- I feel it has aided in my growth as a teacher and enabled me to make success a possibility for my students.

- I feel students greatly benefit from the year-round education calendar. The times at which breaks occur seem to fit just perfectly with student achievement. This break allows students time to renew themselves and also allows time for students who need time to catch up.

- I feel the year-round school calendar provides the students the opportunity to retain their learning better.

- I feel there is a great deal of personal and professional benefits to the yearround calendar. It creates a situation where we have fewer weeks that are broken up by holidays. The intersession opportunities during our between quarter breaks are great.

- I hate it!!!!! The superintendent said we would "revisit" this decision. She manipulates the information Given to her so that the board does not hear what the teachers are really saying. We need to start later and get out before June 1 .

- I have been able to work with small groups of students during the breaks to give them additional opportunities to learn the material we have covered in class. I enjoy having the extra time to spend with my family.

- I have enjoyed the year calendar it offers benefits beyond the regular classroom. One advantage it provides teachers an opportunity to regroup at the scheduled breaks and make adjustments with the curriculum.

- I have enjoyed the year-round calendar as a teacher, as well as from a parent's point of view. The students seem refreshed and ready to work when they return. Personally it provides additional opportunities for vacations. While we all enjoy the time off,

- I have enjoyed working on the alternative calendar. I feel that it helps cut down discipline problems. About the time that students and teachers get really tired and frustrated, they get a break and come back refreshed again. 
- I have found it to be a really good thing. At first I was opposed to the change, but I find that the fall break falls when the first round of burnout used to hit, both for teachers and students. The drawback is starting so early in August when the weather is hot.

- I have honestly enjoyed working in a school system that has year-round education.

- I have never taught in the traditional school calendar; therefore, I really don't have a lot to compare my experiences to. I feel that a year-round calendar has allowed me much needed breaks throughout the school year.

- I have no problem with it. the students need as much time in school as possible. I am still adjusting to the teaching responsibilities and am frustrated with the student lack of care and some behaviors.

- I have not ever taught on a year-round schedule and don't want too.

- I have not had the opportunity to get employment in the traditional summer vacation time. This limits my ability to create income when I am unemployed. Teaching is a wonderful job and I would hate to have to leave it and go to the private sector.

- I have not seen any educational benefits with the year-round calendar. This is from both my students and my own personal children. I believe that the learning environment, including teacher morale, makes the greatest impact on student achievement.

- I have only taught in a year-round calendar, but when I think of how exhausted I am by break and how refreshed I feel after break; I can't imagine teaching in a traditional calendar.

- I have worked on several year-round AND alternative schedules (4X4, alternating day block, standard semesters with mini-courses at breaks) and I see the advantages and disadvantages of each.

- I like it. I like the fall and spring break. However, I would rather just have one week during the break instead of two. This would allow school to be completed by the end of May instead of running into June. I do think we could start a little later.

- I like it. It gives everyone a fresh new start each quarter.[School staff, Parents and students|

- I like major breaks throughout the year. We have adjusted the calendar so that we don't start until Aug. 1 and get out at the end of May. This is much better than starting in July and not getting out until mid-June.

- I like the alternative calendar which allows for breaks to be spread out during the year rather than such a long time off in the summer. This helps children to retain more of what they have learned and less reviewing of skills when school begins in August.

- I like the modified year-round schedule that we have here in Bardstown. I enjoy being out of school in June and July. I would be less content if we reverted back to the calendar of 4-5 years back when we went to school all the way into mid June. 
- I like the time I can spend with my family. I would prefer to only have one week off in Fall, two weeks for Winter and one week in Spring.

- I like the year-round calendar. I think that the frequent breaks help relieve stress associated with teaching and learning for both teachers and students. It helps people recharge and come back fresh and ready to learn.

- I love our calendar. It gives breaks when we need them, without staying out of school so long that the students lose some of their gains before they get back to school.

- I love the 2 weeks breaks, they come just when it seems like everyone is tired and needing a break. The summer break is long enough to where everyone seems to get ready to come back to school.

- I love the breaks being two weeks long. However, the summer is not long enough. It seems the students aren't ready to learn when it is still so hot outside. They also have trouble going to bed early during the first part of school due to the sun not going down.

- I love the calendar.

- I love the two week break to refresh my spirits and prepare for the nest nine weeks. We all need the break, students and teachers alike.

- I love the year-round calendar in that it gives the students and teachers a nice break between 9 week sessions.

- I love the year-round calendar. I think that the calendar impacts the school in a positive way. The 2 week breaks come when students and teachers need a break. I feel that after a break, the kids and I come back ready to learn.

- I love the year-round calendar. I think the breaks give students and teachers the opportunity for down time without risking the loss of knowledge that an extended summer can have.

- I LOVE WORKING AT THIS SCHOOL. I REALLY LIKE THE YEARROUND EDUCATION CALENDAR.

- I only have taught on a year-round calendar and I would hesitate to go to a traditional calendar. Personally it has helped me avoid burn out during the school year and professionally I think it is best for student learning.

- I personally don't think that the type of calendar matters when you consider other essential factors. A progressive administration that leads, is qualified in all areas, and creates pride through requirements for all students and facultycan have a success.

- I prefer the year-round calendar to the block scheduling. I feel like there is more time with the students. I have not been very successful at classroom management in the public schools, having come from Catholic schools.

- I really don't think the calendar has much to do with it. Teachers are frustrated with all the testing and time taken away from quality teaching and planning.

- I really enjoy the 2 -week fall break. This, to me, is the greatest advantage of the year-round school. I would like to address some student issues. At our school $99 \%+$ of the students are very courteous, caring and give a good faith effort when allowed. 
- I really enjoy the year-round calendar, and can't imagine going back to the traditional school calendar. I believe it helps both the students and the faculty get refreshed and fully prepared for the coming semester. Our short summer gives us ample time to prepare for the next year.

- I really like how it is spaced out (intersession, breaks, etc.). I feel that too much time during summer is detrimental for the students.

- I really like the year-round calendar.

- I really like this schedule.

- I see advantages in students having longer breaks between semesters (two week break every nine weeks) and a shorter summer vacation. There is more relief during the school year for both the students and their teachers, and less time to forget newly acquired skills or learning.

- I think it allows for more continuity for the students. Actually I don't think it goes far enough. I think the summer break should be shortened to a month while the winter break should be lengthened to a month.

- I think it is great.

- I think that by the time a break comes; both students and teachers need it. I can't imagine how teachers taught on a traditional schedule. The breaks allow time for planning and re-analyzing to meet student needs. I like the year-round calendar.

- I think that the year-round calendar is wonderful for students and faculty. Students have the opportunity to "get caught up" and reinforce skills during intersession. It is also a wonderful opportunity to offer creative classes for students to attend.

- I think the calendar is good for all. The instructional segments are broken up enough to give a good mental rest.

- I think the year-round calendar is great. It helps break up the school year a little bit better, and now that I'm used to it I don't think I'd want to work on a traditional calendar.

- I think the year-round education calendar is a wonderful thing.

- I think there are too many variables in addition to the year-round calendar that have changed in the years since we went to it. I do not spend the 1st month of school reviewing, which is what common "way back then" but I was review when appropriate.

- I truly enjoy working in a district that uses the year-round calendar. I personally like to refer to it as an alternative calendar since we do get a longer summer break. I believe the breaks are good for our students and they seem to be ready to learn when they return.

- I work with nine schools in a school district as Artist In Residence. My answers to the questions listed reflect an overview of all the schools I attend, not just one particular school. The year-round calendar has allowed me the 
flexibility to arrange my schedule.

- I would like to see the school year begin in middle to late August. Cut fall/spring break to one week and have closing day near the end of May. The two week breaks are a hardship on Many of the families at my school.

- I would rather have a traditional calendar and have a longer summer break; because I usually take classes or staff development over the summer and then there is no time left. Also the two week breaks are a little to long, especially during the time of year.

- Improved burn-out, enabled me to schedule appointments during breaks and gives me time to plan.

- Improved my motivation as well as my students. It has also helped students who are failing because they may make up work during breaks.

- improved student performance

- Improved the consistency of instruction and structure for the students. I have more continuous opportunities to work with students and keep them on-task with learning.

- Increased motivation of students and decreased student/teacher burn-out. I feel that this calendar, along with our 6 period days, has Provide our system with the tools necessary to foster student development and high academic achievement.

- increased overall student retention, especially over the summer break; gives younger elementary students breaks at times when they are beginning to fatigue and become overwhelmed;

- is beneficial to students and teachers.

- Is the best choice

- It does make attendance for me a little better. I can plan appointments easier with this type of schedule.

- It gives us plenty of time in front of the student each year. All parents and schools should teach accountability to the students by requiring them to learn. I believe we as a society have lost that segment of learning.

- It gives ample time for instruction and remediation during intersession.

- It gives me an opportunity to rest and "get my second wind". It helps to recharge my batteries and I feel that the same is true for the students. They seem eager and ready to return to school after one of our breaks.

- It gives me an opportunity to rest and re evaluate the performance and needs of my classroom. Because I am more relaxed and rested, I have an opportunity to think of new strategies that can foster learning. I have an opportunity to take care of appointments.

- It gives more frequent breaks which help we as teachers to refuel. 
- It gives my students (young primary) the chance to take a short break and get refreshed to start a new quarter. They get so tired, especially early in the school year. They need the breaks to keep the attitude positive for learning.

- It gives students and teachers several breaks throughout the year to get refreshed. The summers are still long enough for vacations. Fall vacations are also a plus during the Fall break.

- It gives the faculty and students a chance to recoup physically and mentally several times a year. I feel attendance was better and behavior was better especially in the first 5 or 6 years that we implemented our calendar.

- It gives the students and teachers needed breaks from the pressures of performing as well as give the teachers and students a break from one another. It also shortens the length of the summer break which can be too long in a traditional calendar.

- It gives the students breaks during the year when they are getting close to burning out. They usually come back refreshed and ready to learn. The short summer is beneficial, but has it drawbacks as well.

- It gives you opportunities to continually reach the students. The breaks offer a time for the students and teachers to recharge their batteries. It also gives the students, who are not doing so well and need extra help to receive that benefit.

- It has allowed me to spend 3 weeks at a time at home with my daughter. I am able to travel on the breaks with my family. I am able to physically and mentally take a break from my job!

- It has been a great stress reliever. When your children are become frustrated you get a break and then come back together to regroup. They come back with new minds and are read to move forward.

- It has been a success for the most part. The features of this calendar I least like are that, when we have a two week break, that means we need to finish covering whatever we are studying before we leave. That fact sometimes rushes me as a teacher.

- It has been an easy adjustment to make from a traditional setting. I feel that good teachers can make the change very easy.

- It has been better than the traditional calendar because of the breaks between each semester.

- It has been fine.

- It has been GREAT! The kids (and teachers) need a break every now and then.

- It has been great. It allows me to use one week to focus on the next 9 weeks and it allows me to get caught up from the previous nine weeks and finalize all grades. The other week I try to devote time related my home - getting caught up on projects that I have delayed.

- It has been wonderful! I taught for two years at a school with a traditional calendar. I find the breaks throughout the school year to be great not only for me but also the students. I also find that I don't spend as much time at the beginning of the year reviewing. 
- It has given me the opportunity to get the needed break from the hectic pace of teaching second graders. I'm refreshed and ready to go back to the classroom after a break. It gives me time to reflect and make needed changes in my classroom.

- It has given me the opportunity to take a much needed break from the hectic schedule of teaching second grade. I return after the break refreshed and excited about teaching. It will help me stay in the classroom. It gives me the time to reflect on what I have taught.

- It has given the students a chance to retain more material. For the younger students it is crucial that you review often, and with the year-round calendar, the students retain more.

- It has helped me physically and mentally. It allows us to take a break every nine weeks in order to relieve some stress. I come back, after the break, refreshed and ready to teach again. I also believe that the students perform better because they are rested.

- It has not been much different than when we had a traditional calendar. We just begin about two weeks earlier and have a break in the fall. The fall break is great!!!

- It is a benefit to the students and teachers. The students need a break but have the opportunity to continue learning through intercession if needed for half days.

- It is great! Teachers are rested and students are eager to come to school.

- It is nice to have breaks every 9 weeks. It gives closure to units and a chance for students to catch up. Having the break also helps adults plan or replan instruction.

- It provides students and teachers with an opportunity to regroup and refuel. As a primary teacher, I was concerned with the need to reteach many concepts following the multiple breaks. I couldn't have Been more wrong!

- It relieves teachers and students from getting "burned out" from instruction. At the same time, it allows for remediation for students who need one-on-one attention. There is still plenty of summer left to spend with your own children as well as summer vacations.

- IT SEEMS TO BE A SMALL DIFFERENCE WITH THE OTHER.

- It's a great opportunity to students who need one-on-one remediation or an extra boost. During breaks, we are able to do this. It also keeps teachers from getting burned out and students are not always being pressured. They too get a break.

- It's got pros and cons, just like a traditional calendar. I personally, like the breaks during the school year. I think it gives not only the teachers a chance for a rest, but also the students and everyone deserves a break.

- I've enjoyed longer vacations with my family in the fall and spring. I believe a week in the fall, two weeks in winter and one week in the spring would be best, however. Parents would not have to worry about babysitters as much. 
- JW, I will be retiring in June of this year so that is why some of my answers are the way they are. Thanks and Good Luck with the Doctor's Degree program.

- Kept me fresh, for there are frequent breaks. Teachers receive very little outside motivation or perks (even in this survey you are rewarding the principals, not the teachers). More frequent breaks give me more of an incentive to do my best because I can relax during the break.

- Less discipline problems and students also retain more.

- Let me and the kids have time away and want to get back to school as a group. Rat5her than dreading the end, we look forward to getting back.

- Made it confident for vacations and it gives not only the students a break, but it also helps the teachers to relax.

- Made it easier to get through the year. When you finish a grading period you can look forward to a little time off to recharge your batteries and come back fresh.

- Made it more difficult to cover all the material required before CATS testing. It is impossible to teach four quarters worth of curriculum during the course of three quarters, which is what I'm expected to do on the alternative schedule.

- Made it possible for me to be both a mother and an educator. It gives me time with my family and the breaks are perfect times to reenergize and prepare for the next semester.

- Made it possible to have much needed breaks throughout the year. This helps to eliminate the distinct possibility of teacher burn-out.

- Made me feel like we don't have any time off. Although we have scheduled two week breaks, the amount of work to be completed forces the majority of us to come in during the breaks to try to get everything done.

- Made me realize how difficult it is to teach to each and every student. In the YRE calendar the students are in school for an extended amount of time. This makes it difficult to reach the students after an extended amount of time.

- Made me take a second and sometimes third look at the material that I teach. In the past I have used various games and simulations to teach, but on this schedule, I have found that I have had to leave most of these out.

- Made my job easier and more relaxing. I am able to relax on breaks and get work done for my classroom.

- Made my life more hectic than expected. As a proponent of year-round scheduling, I was surprised to find that I did not like teaching on the year-round calendar as much as I originally thought I would.

- Made my performance as an educator better. I especially like the 2 week breaks that are an essential component of the alternative calendar in my school district. I am convinced that I am healthier (mentally and physically) due to the alternative calendar.

- Made no difference. I still enjoy teaching.

- Made recoupment easier for my students on specific skills. It gives the students and myself a break that brings us back ready to work and progress. 
- Made the job easier. We know a break is coming soon, and the year speeds by. With the traditional calendar, the second semester dragged along toward spring. I have taught as long as I have because of this calendar. Personally, I would recommend it to other teachers.

- Made the year seem more adapted for kids to learn. Not having the two months to "forget" things has made a big difference. I always review after a 2 week break and then we are ready to continue with new info.

- Many advantages over the traditional calendar

- Many advantages that allow the students to stay more in-touch with the curriculum and the classes that they take. I feel that the two week breaks are great because they give teachers and students a chance to recharge their batteries and get ready for the next quarter.

- Many advantages to educators and students alike. Students seem to retain more information from the end of school to when the new year begins. The breaks are just enough to keep everyone motivated to return and learn. $\mathrm{T}$

- Many advantages to student learning and keeps teachers refreshed so that we can better serve our students.

- Many advantages. The breaks that we receive throughout the year are well needed by the staff and the students. I enjoy the breaks throughout the school year and I know that the children do as well. I believe those break allow the students to relax and rejuvenate.

- Many benefits! I enjoy getting the extra breaks; it gives my family flexibility in planning vacations, etc. It also gives my children the opportunity to have breaks within the school year! Long summers are hard on children to keep their skills.

- Many benefits. As a parent, I can spend quality time with my children over the two week breaks and summer break. Professionally, I can reenergize and be ready for the next quarter or school year during the breaks.

- Many benefits. I feel refreshed after a break. The break gives me an opportunity to plan fun, innovative lessons for my students.

- Many benefits. I would not work in a district that did not offer the year-round calendar. I can not imagine not having the breaks to plan. The students come back refreshed and ready to work. I recommend the year-round calendar.

- Many benefits. Students enjoy a rest between quarters and the summer is long enough for students and teachers to refresh and recharge. Summer break is also long enough for teachers to prepare for the next year.

- Many benefits. We get a nice break about every nine weeks. I think this benefits everyone involved. The staff gets a small break and is able to come back refreshed several times throughout the year. The students come back refreshed after every break.

- Many positives and a few negatives. The negatives focus on high school issues specifically related to 9 week terms and instructional time. Some of the natural breaks during the year do not accommodate a term and we have shortened instructional time periods. 
- Meant an opportunity to enjoy time off during each season of the year, opportunities to take advantage of professional development and not worry about planning for a substitute, working with students who present a lesser degree of regression due to a short break.

- More benefits than drawbacks. However, the 2 week breaks are too long for the slower students who need the constant reinforcement.

- More benefits. I am able to spend more time spread throughout the year with my three year old son and do educational and worthwhile activities with him. I'm sure Many other parents do similar things.

- More disadvantages than advantages.

- more positive attributes than negative ones

- Most of my teaching has taken place in schools on the year-round calendar. I feel like the students do not regress as much over the summer as students who are on a traditional calendar. I feel like there is less review necessary at the beginning of the school year.

- Most of the negative answers from my survey are a result of numerous administrative changes which have caused a disarray of vision and discipline for our school. Younger teachers are not receiving support they need to be successful.

- Motivated both me and my students because it gives us a rest. It has also helped students who are failing to make up work.

- My least favorite part of a year-round school year is that our spring break is in March; hence, it is two weeks out of the portfolio process. I also don't like that our summer is much shorter.

- My negative feeling toward year-round education stem from the hardships that I think it brings on students and teachers that are involved in extra-curricular activities that force them to lose portions of their break.

- Not been a bad situation in either aspect. The year-round calendar is a refreshing change from the traditional calendar. I enjoy it a great deal.

- Not Been a problem for me in terms of adjusting my teaching style. I still would prefer a traditional school calendar. It would be a tremendous help for schools and students if all schools were on the same calendar to help with transfer grades.

- Not changed much the way things are done. Parents still remove kids for vacations, hunting .... whatever and whenever they want. Kids miss lots of school. A true YRC is not used, what we do is not as flexible as was proposed.

- Not given a clear indication that it is beneficial. If I could see a real connection to performance and the year-round calendar I think I would be more of an advocate for it. As of now there is no strong correlation with performance and the year-round calendar.

- Not good for Kindergarten. The two week fall break comes at a time when 5-6 year olds are beginning to understand routine and adapt to the new environment. They need to stay in school for a longer period of time before a 
break occurs.

- Not made a whole lot of difference in informational retention with high school students.

- Not made much impact on students at the high school level.

- Not only benefit the students but, also the teachers.

- Not really changed much about the way I teach.

- Offered me a goal to work toward. I know that I have a break every 9 weeks and I hang on until I make it.

- Offered some flexibility professionally and personally. It has allowed an opportunity for challenged students to catch up with their peers, it provides planning time and a rejuvenating period for teachers, and gives families flexibility for vacations.

- On a personally level I enjoy the breaks. I can get motivated again after a long break. I do not have anything to compare to, because I have always taught yearround, but I do not see any disadvantages with my students' education.

- Only provide a good learning environment for special education students who have behavioral disorders. They appear to work better but lose detailed instruction from previous 9 weeks.

- Opened my eyes to how students do not get burned out from the traditional calendar. I also do not get tired as easy due to the breaks. I come in each new 9 week period feeling refreshed. I also do not fall behind in my teaching because each break gives time to catch up.

- Opportunities to go on vacations during other times of the year besides summer. More opportunities to prepare for the next nine weeks. I still miss the longer summers but I do like the fall break. One of my concerns is the spring testing window.

- Opportunity for $\mathrm{R}$ and $\mathrm{R}$ is available and yet not time for appreciable loss of retention by the students. I like the calendar much better than the traditional calendar I taught on for 30 years in Oklahoma.

- Our opening day is beginning to get earlier. This makes the time preparing your classroom in the summer shorter.

- Overall, I found it to be very beneficial all the way around. Especially, Fall Break!

- Personally - Given me time to be with my family and have breaks in the school year to "recharge". Professionally - Given me time to "recharge" during breaks. Given me opportunities to pursue trainings and also time to catch up on paper work and planning the next quarter.

- Personally and professionally the Year-Round Calendar has allowed me not to be as tired, stressed and worn down by the demands of teaching, by the time I have felt this way, I know in a week or two I'll have the opportunity to get some much need rest. 
- Personally and professionally the year-round calendar helps me. Some of the ways are: I plan for the 9 weeks in a block and that helps me know exactly where I am in the content areas, what I have done, and what I need to do.

- Personally- I enjoy the long breaks during the school year. It allows me to visit with family throughout the year. Professionally- It allows for more consistent contact time with the students.

- Personally I enjoy the year schedule. I can plan several vacations throughout the year. It is nice to have breaks in the year. I do feel that students do not achieve as well in this type of calendar, compared to a more traditional calendar.

- Personally it provides much needed breaks to renew myself and prepare for the next quarter. It offers time to make appointments, complete PD, etc. Professionally, it helps my students retain information.

- Personally kept me from having to take "mental health days" around October, November, and February, due to the wonderful fall, winter, and spring breaks. It has also Given me the flexibility to travel during non-peak travel times.

- Personally provide me the opportunity of working really hard and diligently through the year for 9 weeks at a time, then provide me with a 2 week break to rest and become inspired to plan and do my best for the next 9 weeks.

- Personally the year-round calendar has Provide me the opportunity to enjoy the parenthood stages with my children (feeling like a stay home mom) as well as giving me the opportunity to feel like I am providing a career for the use of leading children toward learning.

- Personally, I enjoy the year-round calendar. It gives me more flexibility than the traditional school schedule. I have taught during Intersession for remedial reading and math. $T$

- Personally, I would prefer to have one week off for fall break, 2 weeks off at Christmas, and one week off at spring break. I would like to start back later in August.

- Personally, it has allowed me opportunities to do things with my family that could not be done during the summer. I enjoy the two week breaks that we get during the year. It gives me a chance to energize myself both mentally and physically to do the kind of things I like.

- Personally, the alternative calendar has been wonderful since it has allowed for family travel at various times of the year when prices are more economical. It allows for a time period to explore other activities and to relax from the stress of teaching.

- Personally, the one thing that's Been frustrating is my two sons are in a district with a traditional calendar so our breaks never are the same, and we started almost a month before them so family time off together and family vacations have Been difficult.

- Personally: -I clean house well four times a year. ;-) -time for self and family, allowing me to recharge for the next quarter; professionally: -clearly defined quarters of learning, able to reset/restructure materials, classroom and other resources accordingly. 
- Prevented burnout.

- Prevented me from doing a lot of family activities on the West Coast, as they begin school at the end of August or after Labor Day. I would like to see the calendar changed until later in August, and the testing dates moved back so the appropriate amount of time before the test is greater.

- Problems with meeting the needs of my students. When there are extended periods of time without school throughout the school year, I have to reteach so Many things to get everyone back to the level I expect of them.

- Professionally, I believe the opportunities to save failing students are undisputable. We offer intersession during Fall and Spring breaks. Many students would undoubtedly fail for the year if they did not have these remediation opportunities.

- Professionally: Has afforded me the opportunity to attend all-day PD sessions I might not have been able to attend. It has given the students time for rest AND remediation. They come back from breaks ready to learn. Personally: It allows me time with my family.

- Professionally, initially it allowed us to reach students throughout the year, rather than waiting until the end of the year, to try to get them caught up on concepts and/or missing work. Now, we focus on open-response questions, so it allows us more opportunities for practice.

- Proved to be a nice experience. I think the breaks are good for the students and their performance.

- Proven to assist the students at the high school level more than at the elementary level. Elementary students loose gained skills over a two week and three week time period, and the time necessary to prepare adequate review for the CATS test is limited.

- Proven to be effective.

- Provide a better teaching environment. I see a positive affect on the student and teacher morale with the extended breaks throughout the year. With each break comes an opportunity for students and colleagues alike to start over.

- PROVIDE A GREAT ENVIRONMENT FOR TEACHING. I LOVE THE YEAR-ROUND CALENDAR!

- Provide a much needed break for students while allowing them to have a "fresh start" after each break. Students come back refreshed and ready to learn.

- Provide a short-term calendar for weaker students. These students can visualize ONE 9-week period and then attend intercession to catch up at the end of each quarter (if that is needed). It is unrealistic to think that weak students can catch up at the intersessions.

- Provide adequate breaks which, although often requires remediation for students, helps to rejuvenate the students and staff.

- Provide an opportunity for our students to retain information better. Our population is approximately $48 \%$ English-second-language learners, and it has Been an advantage to have the block of time that they are away from the 
educational process shortened.

- Provides an opportunity to "revamp, recoup, reevaluate."

- Provides clear delineation of quarters, which make it easier to plan for instruction, to craft different kinds of assessment within each quarter; Provide breaks during which I can catch up on grading (Many essays), evaluate instruction, plan for and

- Provides enough breaks during the school year to keep most teachers as well as students from becoming burnt out as the school year progresses.

- Provides good opportunities after each 9 weeks to reevaluate instruction, and make changes in room arrangement and appearance.

- Provides great opportunities for the school. Over our two week breaks, we offer camps to help the learners that are struggling. I feel this is a great opportunity. Also, when they come back in the beginning of the year they are not as "out of it", because they are rested.

- Provide longer and welcomed breaks throughout the year, but has not proven to me to produce better learning in the students.

- Provides me with a break when I become frustrated or burnt out.

- Provides me with a chance to 'recharge my batteries' and opportunities to get caught up as well as plan ahead for the next quarter.

- Provides me with an extra income when I teach the interim classes and allowed more time to rebuild my energy levels to come back much more ready to do a good job!

- Provide me with breaks that I need to create instructional units and activities to meet the needs of my students

- Provides me with breaks to catch up on organization/paperwork/less important duties that have been put on hold, offer extra camps for students during the breaks, given me a time to rest and get refreshed

- Provides me with extra time to work on providing extra time to work on activities and strategies to help my students. I also spend my "off" time working on my classroom to provide a more unique place for my students to learn.

- Provide me with frequent breaks which are really needed. I have found that when the breaks roll around the children are really ready for the break as well as the teachers. I believe everyone comes back ready to work.

- Provide me with the opportunity to have a break frequently throughout the school year and has allowed my family the opportunity to spend time together at non-traditional vacation times. Professionally the year-round educational calendar allows students to have more time to master learning.

- Provide me with the opportunity to relax, refresh, and develop new, creative ideas and strategies to meet the needs of my students. I have been teaching students with special needs for thirty years, and I look forward to the breaks in order to have time regroup and refocus. 
- Provides me with the opportunity to be both a single mother as well as a professional. I still get the satisfaction of being a stay at home mom 12 weeks a year, and the self esteem boost of being a working women. It keeps me from getting burnt out on teaching

- Provide needed breaks throughout the school year. This helps my students and me maintain a smoother energy level. The breaks provide time to reflect and process.

- Provide opportunities for remediation between nine week periods. The calendar also provides much needed breaks for students and faculty to "recharge".

- Provide opportunities, researched based opportunities, to enhance and increase student achievement. The intersession time is a GREAT tool, if used appropriately.

- Provide students with a good education. They enjoy going to school but having weeks and days off throughout the year to rest and spend time with their families. I enjoy the year-round calendar for these same reasons. I prefer this better than the traditional calendar.

- Provide students with sufficient breaks while still allowing students to learn and retain key knowledge in the various academic areas to make them successful.

- Provide the much needed break to refresh and refurbish for both students and teachers. It also provides intersession opportunities to remediate and enrich students academically.

- Provide the needed intermittent breaks that serve to refresh and renew, as opposed to being so worn out by summertime that it would take several weeks just to "catch up." Additionally, our family enjoys the alternate of times during the calendar year to vacation.

- Provide the opportunity for me to reinforce many concepts that are difficult for students to grasp. However, on a 4 x 4 block, students who take foreign languages do not have the same exposure time to the languages as those students who follow the 6-per teacher.

- Really not made that much of a difference. I have worked in other school systems that have had a traditional school year. I feel that the biggest difference is that when there are two week breaks rather than one, it takes longer to get the students back on track.

- Reduced the amount of learning that is lost over traditional summer periods.

- Seemed to benefit student achievement, student focus, and is a positive change for education.

- Served my purpose well

- Shortened my summer. It requires that school begin too early in August. I would prefer that we start school later in August, even if that means going through mid to late June.

- Shown me that students do better with breaks throughout the year, but not such a long summer break. It is easier to get students "back on track" when they are 
not out of school for long periods of time, as is the case with a more traditional calendar.

- Since this is the model I have worked under through most of my career, I have very little to compare it with. I think it has Been effective. However, I do not believe that one week sessions of intersession sprinkled throughout the school year really helps.

- Some benefits such as Intersession. However, we come back to school too early.

- Some good advantages.

- Some good points and some bad points. As far as retaining what has Been learned, I see know signs that students are able to remember what has Been learned from one vacation to the next no matter how long or short.

- Students in unairconditioned gyms, classrooms, and schools trying to work and complete the necessary skills in the middle of the hottest weather are neither practical nor healthy.

- Taken time to get use to. However, I like the fact we have time off after each 9 weeks. It gives me time to prepare for the next 9 weeks. (get new ideas, supplement with new materials etc.) As for the students, it gives them a break.

- Thanks

- The calendar has allowed me time each nine weeks to have time to renew my energy and enthusiasm for teaching. I also spend several days during the days off working in my classroom so that when I return to school I am better prepared to offer my students

- The calendar has been great! I love getting a two week break every nine weeks. It gives the kids and teachers a nice break.

- The calendar has Many advantages: -kids come back from breaks calm and ready to learn; -behavior problems reduced due to fact that they get breaks from each other; -teachers are refreshed and ready to go; -you still get a break in the summer, but not so long.

- The calendar has produced mixed results. It gives teachers and schools ample chances to provide for extended services and intensive interventions. However, the shortened summers seemingly gives some teachers with very little time to "recharge" their battery.

- The calendar is fine it is the behavior problems in the classroom that $\mathrm{i}$ am discouraged about.

- The calendar that our school is on has proved to be beneficial for students with special needs. I have enjoyed having the breaks and having an opportunity to choose when I vacation.

- The calendar is great; provides small periodic breaks throughout the year and students get a better education. They need all they can get. This is my first year and it is tough to adjust to this job but I have no problems with the calendar.

- The only major concern I have with the year-round education calendar is CATS testing in the Spring. We (year-round districts) have the same testing window 
as those who follow the traditional school calendar.

- the potential to be more effective if the community would support true academic excellence. Academic excellence seems to be viewed as the other person's role. I do not believe it is restricted to this community but I believe across $\mathrm{KY}$.

- The year-round calendar for me has been mostly positive. It helps teachers have a break and come back with more positive attitudes. The only drawback is when you have young children (preschool) it is sometimes hard for them to adjust after being off for a while.

- The year-round calendar gives me the opportunity to be reenergized and plan for the next nine weeks. I spend some of the time off from school organizing activities and the learning environment for the next quarter.

- The year-round calendar has been wonderful for me as a mother. I get to spend more time with my kids and I feel like I have more opportunities to plan special events for my family. Professionally, I get frustrated at times with the calendar.

- The year-round calendar has Given me the opportunity to be well rested and prepared for teaching. I like the two week breaks-one week I can relax and the other week I can plan for the next nine weeks. It also allows my family and me to vacation alternate times of the year.

- The year-round calendar has kept me highly motivated and the children come back eager to learn.

- The year-round calendar has not worked as successfully for the students as I at first thought it would. The students that are behind at the end of the quarter will seldom come to the intersession classes held during the break.

- The year-round calendar has proven to be a great asset to me as an adult. However, Having taught about that same amount of time in and out of yearround, I do not see a large advantage for the children.

- The year-round calendar has Provide the children and the staff with breaks at a time when they are needed. It prevents burn out for all. It also provides a time for children to received additional help.

- The year-round calendar helps me not to get burned out. Knowing that I have intermittent breaks to look forward to help me to set goals for myself and students and rigorously work toward them. It provides time to plan and scrutinize lessons that address students' needs.

- The year-round calendar is beneficial for some students in my classroom. It is difficult however to be in a school that has a year-round calendar and have children who attend a school with a traditional calendar.

- The year-round calendar is great it gives students much needed breaks without making it seem like coming back early is a punishment. I also see improvement is the work that is produced because of the breaks that implemented during the year.

- The year-round calendar is GREAT! It gives teachers a break through out the year! They need it!

There needs to be more at school care during those breaks in the calendar for the students. Parents sometime do not have care for the two week breaks. 
- The year-round calendar is the only system I have taught in and I have Been teaching for ten years. Instructionally it makes the most since for the students.

- The year-round calendar is wonderful! I would NOT under any circumstances want to return to a traditional calendar!

- The year-round calendar offers an opportunity to regroup and refresh for the upcoming quarter. It is greatly needed by teachers and students alike.

- The year-round calendar offers students and staff short breaks throughout the year. These short breaks give all the stakeholders time to "recharge" before the next term or next school year.

- The year-round calendar provides me with longer class periods which allowed me to incorporate a lot of LABS in student learning which provides them with an excellent opportunity to practice what it is that we are learning.

- The year-round calendar suits me very well. I especially like the long break in the fall.

- The year-round calendar was a good idea at first with the so Many weeks on and so many off. What we have found out is that the breaks were too long during the year and the summer break was shorten which created a lot of problems for up keep of our buildings clean.

- The year-round calendar's breaks lessen my job stress, give me opportunities to plan more interesting and effective units of study, refresh my outlook with challenging students, and improve my overall job satisfaction. I love it for myself, and I loved it for kids.

- The year-round calendar is good. I really enjoy it, although I wouldn't a couple of extra weeks off in the summer. Still it works out really good for me.

- The year-round calendar is okay, but students do forget a lot over each break and you have to review. I find myself as a teacher doing a lot of work over the break to prepare for the next nine weeks and I don't get a long "mental" break over the summer.

- The year-round calendar personally allows me time to take a short break from the pressures of teaching. The breaks allow me to come back to school refreshed. I'm certain that students feel the same way.

- The year-round education calendar has allowed me to stay excited about my job. Just when the slumps start to come, it seems we get a break. This gives me a chance to re-energize and come up with something new and exciting for the kids.

- The year-round education calendar has been a great experience for me. I like the breaks in the fall and spring because it gives me a chance to spend time with my family and be a mom but it also lets me regain my strength and motivation.

- The year-round education calendar has Provide shorter breaks during the school year for my family to share time as well as step back from the day to day classroom activities. As I return from each break I notice the students and teachers have positive attitudes.

- The year-round education for me personally is wonderful. I enjoy the October break which was not available to me in a traditional calendar. However, I am not sure that the educational benefits are there for the children. 
- The year-round education has Provide opportunities for our teachers and students to focus on learning with the needed breaks to help keep a good focus. I like the alternate calendar because it provides me with the chance to take some vacation time in the fall and spring.

- The Year-round calendar allows students/teachers/staff to "rest and refresh" between chunks of intense learning. I feel that helps them learn to the best of their personal abilities over-all! It helps the student who is of the mind set to learn \& work.

- The year-round calendar is not bad. However, I do not like that it causes us to get out so late in May and start so early in August. In addition, I do not like that, if I am covering something before spring break, it will be two weeks before we return to class.

- The year-round calendar, has allowed the opportunity for me to work with individual students during the breaks. It has Provide me the opportunity to give students a chance to catch up when they've fallen behind before it's too late.

- The year-round-calendar has worked well for our school. The burn-out-rate for students and I suppose even for teachers is not as high as it would be if we were following a traditional calendar year.

- The YRE calendar allows me time to rest and recoup as a teacher and mom, but I still have time during the breaks to come into my classroom and make some changes that benefit the students learning. It also allows me the opportunity to earn extra money by teaching intersession.

- The YRE calendar has proven that it has merits, especially since we have good numbers of students attending the intersessions. The administrators have also proven to me that students will not suffer from not being able to attend summer programs.

- The YRE hasn't really affected me as a teacher. We're still required to be in school the same number of days. In fact, the periodic breaks we get with YRE doesn't supplement retention at all. I find myself retracing my steps with classes to ensure they don't forget.

- There are benefits with the modified calendar that we now use here at Bardstown Ind. I like the calendar that we use today much better than the calendar that we used 5-10 years ago. I enjoy having June and July off of work but also like the fall and spring breaks.

- There needs to be more at school care during those breaks in the calendar for the students. Parents sometime do not have care for the two week breaks.

- There's always room for improvements in areas.

- This calendar has kept both interest level high for both students and faculty.

- This calendar lets me have weeks off to refresh myself and reflect on the school year at that point and make necessary changes. The time also gives me an opportunity to do things with my family. At the point that school has become stressful, it is time for a break. 
- This has been the only calendar I have had. I don't mind it all. The students benefit from the breaks we receive. They come back refreshed and ready to learn again. We as teachers need the breaks also to feel refreshed and relaxed so that we are ready to start the new quarter.

- This is my very first year (actually 4 months) working with this calendar. I think that I will have a better answer after I teach in it for the duration.

- This is the only education calendar I have ever taught in.

- This is the only educational calendar I have taught in.

- Time for me to clear my mind and get mentally ready to go again!

- Time to wrap things up; time to plan; time to relax for everyone - one week for each!

- Too long breaks would like to see one week fall break and only one in spring so we would not have to start school on July 31 . Way too early to start school.

- Very little value. I see students lose just as much in two weeks as they do over the summer. We come back to school when it is way too hot to take the children outside to play which is very important for elementary students. Not all schools have air conditioned.

- Very nice. My family and I are able to take several vacations throughout the year. I am also able to make doctor's appointments and dentists appointments during the breaks. The year-round calendar has also Given me well deserved breaks throughout the year.

- we are on extended calendar - not year-round

- We don't consider our calendar to be "year-round." I do like the alternative calendar under which we operate because it offers more time for planning between 1 st and 2 nd, and between 2 nd and 3rd terms. It also allows my family to vacation at times other than summer.

- With teaching the youngest children, we were concerned. The 9 weeks on and 2 off has Been wonderful. The 7/8 weeks in the Summer has Been a great help with learning retention. I would recommend this to any school system.

- Within the type school I teach in, the breaks come at a time that gives me the opportunity to refresh myself. Without the breaks I think my satisfaction would be much less and would have to look for a new position.

- Wonderful

- Wonderful opportunities to re-group and re-focus during the fall and spring breaks. It gives you the opportunities to take care of the things at home you have had to put on the back burner while school is in session.

- Worked extremely well. I have found that students come back from a short break revived and ready to learn as do the teachers. As for the reason I will be actively looking for a new job, my husband has just gotten a job in Texas, and I will be moving to Texas. 
- Worked out fine. I like having small breaks throughout the year.

- Worked out great

- Worked very well. I like it.

- Worked well with my family environment

- Worked well with vacation times with my family. It also helps break up the year and allows a chance for me to catch up if I get behind or an opportunity to plan ahead during the school year.

- Worked well. I think the two week breaks give the teachers as well as the students a chance to get refreshed and ready for the next round. The breaks also allow the teachers to work with students who are having difficulties at the end of the 9 weeks.

- Year around calendar has many perks until it comes to Winter. It then becomes a worry or pain about when the days are to be made up. Other than that, Year around is a good teaching environment.

- Year-round school provides me with opportunities to plan activities which have real world connections and to evaluate student progress in depth following each quarter. Students who need additional instruction are given more in depth assistance in small groups.

- The Year-round calendar makes teachers more motivated to teach because they are getting lots of little breaks to rejuvenate themselves. The biggest plus is with the students they have time to rejuvenate too but not so much time that they forget the skills taught.

- Provide valuable opportunity to network with staff in order to improve upon my professional knowledge as well as create plans of action in the ongoing school year instead of just in the summer breaks. The year-round calendar provides a much needed respite.

- Been HEAVENLY. The 2 weeks off after each quarter refresh not only the students but the teachers. It is a WELL needed and WELL deserved break.

- I feel students greatly benefit from the year-round education calendar. The times at which breaks occur seem to fit just perfectly with student achievement. This break allows students time to renew themselves and also allows time for students who need time to catch up.

- Been a challenge. I find it hard to find stopping points in my lessons when breaks are about to happen. If I am in the middle of a chapter I have to go back and almost start over because the students don't remember from 2 weeks ago.

- Been a great schedule for my students' retention rates and fine for my "recovery" periods. I like our current schedule; it has just been a difficult experience with our lack of effective leadership.

- Classified employees are not given a chance to go to PD or get promotes as the certified employees. A Gap that needs to be reviewed 
- More disadvantages than advantages.

- Given me a chance to take more vacations throughout the year!

- Been wonderful. There are so Many advantages to this calendar, both professionally and personally. I would not be happy to go back to a traditional calendar.

- Caused me problems in finding summer work to supplement my teaching salary and also made it difficult to complete Rank 1 requirements through summer school (university) courses.

- Been a wonderful experience for both myself and my students.

- Given me an opportunity to have a short break and come back rejuvenated and ready to motivate students to learn. The students are also ready to learn again from the short break but have not forgotten material like they would with longer breaks.

- Been a great and rewarding schedule for the students and teachers at our school.

- It has Helped me physically and mentally. It allows us to take a break every nine weeks in order to relieve some stress. I come back, after the break, refreshed and ready to teach again. I also believe that the students perform better because they are rested.

- Given me restful time off to enable me to be fresh and ready to begin each new quarter. 


\section{CURRICULUM VITAE}

NAME:

ADDRESS:

DOB:

EDUCATION

\& TRAINING:

PROFESSIONAL EXPERIENCE:

CURRENT

1995 -

1990-1994

1984-1990

1980-1990
Joseph W. Mattingly

439 Bloomfield Road

Bardstown, KY 40004

Lebanon, Kentucky - January 26, 1953

Doctoral Program, University of Louisville, 1995 - Present

Rank I, Supervision \& Principalship, University of Louisville, 1980-1983

M. Ed., Education, University of Louisville, 1977-1979

B.A., English, Warren Wilson College, 1973-1975

B.A., Social Studies, Warren Wilson College, 1973-1975

A.A., St. Catharine College, 1971-1973

Associate Superintendent, Technology CIO, Coordinator of Titles I, II, II; Part D, IV, V, KERA Preschool, Technology, Comprehensive District/School Improvement Plans, School Report Cards, Member of Construction Committee

Supervisor of Instruction: Coordinator of Title I, II, IV, VI, KERA Preschool, Technology, Comprehensive District/School Improvement Plans, School Report Cards.

Supervisor of Federal Programs: Coordinator of Chapter I, II, Title II, V, KERA Preschool, Technology, IDEA, Preschool Handicap, Coordination with HeadStart.

Assistant Principal at Bardstown High School.

Language Arts Teacher at Bardstown High School, Boys Track Coach, Assistant Boys Basketball Coach. 
Language Arts and Social Studies Teacher at Bethlehem High School, Girls Basketball Coach, Boys/Girls Track Coach.

AWARDS:

Bardstown High School Athletic Hall of Fame 2005

International Who's Who of Professionals 2001

Kentucky Educational Leadership 1992

Bardstown/Nelson County Leadership 1990

PROFESSIONAL SOCIETIES:

CIVIC

ORGANIZATIONS:

NATIONAL

MEETING

PRESENTATIONS:

INVITED PRESENTATIONS:
Kentucky Association of School Administrators

Region 5 Kentucky Association of School Administrator

Kentucky Association of Supervisors of Instruction

Region 5 Association of Supervisors of Instruction

Kentucky Association of Technology Coordinators

Central Kentucky Association of Technology Coordinators

International Society of Technology Educators

Bardstown Rotary International

Stephen Foster Drama Association

National Youth Sports Coaches Association

Kentucky State Coaches Association

Bardstown/Nelson County United Way Board, Former Director

2004 Presented "Study of Year-Round Education in Select

Kentucky School Districts at the National Year-Round Education

Conference, San Diego, CA.

2003 Presented "Study of Year-Round Education in Select

Kentucky School Districts at the National Year-Round Education Conference, San Diego, CA.

2000 Presented "Challenges of Change" at National Year-Round Education Conference at Las Vega, NV.

2000 Presented "YRE: A Critical Change Agent for Secondary Schools" at National Year-Round Education Conference at Las Vega, NV.

2005 Presented "Year-Round Education: Bardstown's

Nine Year Journey" at Kentucky Association for Year-Round

Education State Conference, Eminence, Kentucky

2004 Presented "Year-Round Education: The Alternate

Calendar!" at Beaver Valley Intermediate Unit Cooperative,

Aliquippa, PA.

2001 Presented "YRE: A Critical Change Agent for Secondary Schools" at the Kentucky Association for Year-Round Education Conference, Bardstown, KY. 
1999 Presented "How Well Is It Working? The Latest Research on Year-Round Calendars" at the Kentucky Association for YearRound Education Conference, Bardstown, KY.

1999 Presented "How Does YRE Impact Instruction and Management" at the Kentucky Association for Year-Round Education Conference, Bardstown, KY.

1996 Presented "Planning and Implementing Year-Round Schooling" at Morehead State University: "Great Schools for a Great Kentucky" sponsored by Morehead State University and Prichard Committee for Academic Excellence.

1996 Kentucky School Board Association (KSBA) state conference: Presented: A Planning Guide for Studying Educational Initiatives in the Local District.

1995 Participated in Murray State University "Year-Round Schooling" Conference.

1995 The Prichard Committee Annual Meeting - Participated on a Discussion and answer planning concerning: Task Force on "Restructuring Time and Learning."

1995 Kentucky School Board Association (KSBA) state conference: Presented: A Planning Guide for Studying Educational Initiatives in the Local District.

1995 Regional Service Center Associates (RSCA) $1^{\text {st }}$ Annual State Conference - Presented: A Planning Guide for Studying Educational Initiatives with special emphasis on effective methods that use the training provided by the RSCA as applied to studying Year-Round Education.

1994Graduate school presentation: "Restructuring Schools: Beginning with Finance."

1994 Graduate school presentation: "The Strategic Planning Model as Applied to the Bardstown City School's Comprehensive Plan for Technology."

1994 In-service with Cinda Stearns for Bardstown High School: "Planning Unit and Lesson Plans Aligned with KERA Transformations" 
1993 In-service for local principals: "The Role of the Schoolbased Admission and Release Committee (SBARC) Chairperson"

1993 In-service Bardstown High School: "Student Transition Planning and monitoring"

1991 Leadership: Bardstown-Nelson County: Presentation; "Education in our Community" 